\title{
ADAPTIVE RELIABILITY ANALYSIS OF REINFORCED CONCRETE BRIDGES USING NONDESTRUCTIVE TESTING
}

\author{
A Dissertation \\ by \\ QINDAN HUANG
}

\begin{abstract}
Submitted to the Office of Graduate Studies of Texas A\&M University

in partial fulfillment of the requirements for the degree of

DOCTOR OF PHILOSOPHY
\end{abstract}

May 2010

Major Subject: Civil Engineering 


\title{
ADAPTIVE RELIABILITY ANALYSIS OF REINFORCED CONCRETE BRIDGES USING NONDESTRUCTIVE TESTING
}

\author{
A Dissertation \\ by \\ QINDAN HUANG
}

\author{
Submitted to the Office of Graduate Studies of \\ Texas A\&M University \\ in partial fulfillment of the requirements for the degree of \\ DOCTOR OF PHILOSOPHY
}

Approved by:

Chair of Committee, Paolo Gardoni

Committee Members, Stefan Hurlebaus

John Mander

David Rosowsky

Simon Sheather

Head of Department, John Niedzwecki

May 2010

Major Subject: Civil Engineering 


\begin{abstract}
Adaptive Reliability Analysis of Reinforced Concrete Bridges Using Nondestructive Testing. (May 2010)

Qindan Huang, B.S., Tongji University;

M.S., University of Toledo

Chair of Advisory Committee: Dr. Paolo Gardoni
\end{abstract}

There has been increasing interest in evaluating the performance of existing reinforced concrete $(\mathrm{RC})$ bridges just after natural disasters or man-made events especially when the defects are invisible, or in quantifying the improvement after rehabilitations. In order to obtain an accurate assessment of the reliability of a RC bridge, it is critical to incorporate information about its current structural properties, which reflects the possible aging and deterioration. This dissertation proposes to develop an adaptive reliability analysis of RC bridges incorporating the damage detection information obtained from nondestructive testing (NDT).

In this study, seismic fragility is used to describe the reliability of a structure withstanding future seismic demand. It is defined as the conditional probability that a seismic demand quantity attains or exceeds a specified capacity level for given values of earthquake intensity. The dissertation first develops a probabilistic capacity model for $\mathrm{RC}$ columns and the capacity model can be used when the flexural stiffness decays nonuniformly over a column height. Then, a general methodology to construct probabilistic 
seismic demand models for RC highway bridges with one single-column bent is presented. Next, a combination of global and local NDT methods is proposed to identify in-place structural properties. The global NDT uses the dynamic responses of a structure to assess its global/equivalent structural properties and detect potential damage locations. The local NDT uses local measurements to identify the local characteristics of the structure. Measurement and modeling errors are considered in the application of the NDT methods and the analysis of the NDT data. Then, the information obtained from NDT is used in the probabilistic capacity and demand models to estimate the seismic fragility of the bridge. As an illustration, the proposed probabilistic framework is applied to a reinforced concrete bridge with a one-column bent. The result of the illustration shows that the proposed framework can successfully provide the up-to-date structural properties and accurate fragility estimates. 


\section{DEDICATION}

To my family and my fiancé, Dan Ocharzak 


\section{ACKNOWLEDGEMENTS}

I would like to thank my committee chair, Dr. Gardoni who advised and supported me throughout the course of this research. Particularly, Dr. Gardoni put a lot of effort in helping me improve my oral and writing communication skills and gave me many opportunities to prepare myself to pursue an academic career in the future. My committee members, Dr. Hurlebaus and Dr. Mander deserve considerable thanks for providing advice and mentoring. Their advice to me as I began my transition from a graduate student to a professional is valuable. I would like to thank my committee members, Dr. Rosowsky, and Dr. Sheather for their guidance and supports.

Thanks also go to my friends and colleagues and the department faculty and staff for making my time at Texas A\&M University a great experience. I also want to extend my gratitude to my friends in the Mandarin Grace Bible Church (College Station, TX) and the MOSAIC group in Columbus Chinese Christian Church (Columbus, OH) who support my study at TAMU and always remember me in their prayers.

More importantly, I would like to thank my parents, my sister, and my fiancé, Dan Ocharzak. Without their support and great encouragement, I cannot concentrate on my work and overcome the difficulties during these years.

Finally, thanks to God who gave me this experience at TAMU and gave me the strength, wisdom, and perseverance to achieve all that I have achieved. 


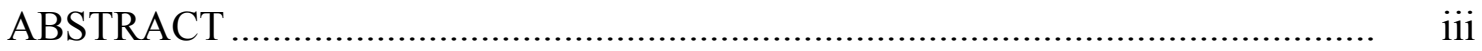

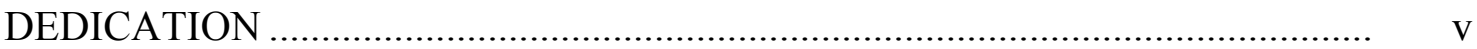

ACKNOWLEDGEMENTS ........................................................................ vi

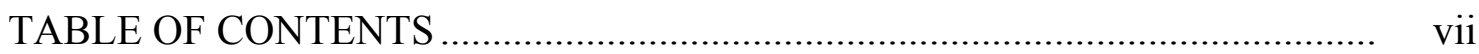

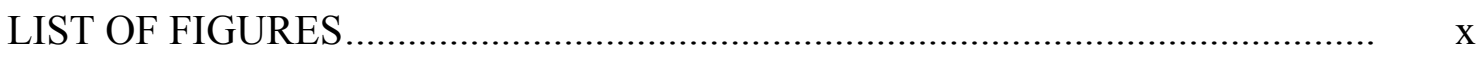

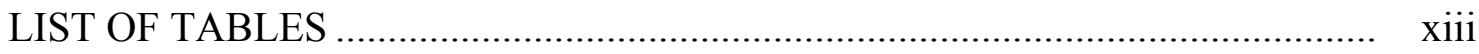

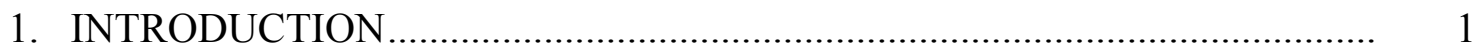

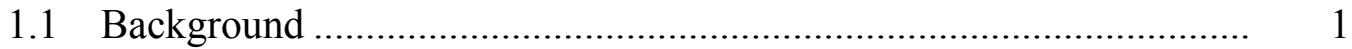

1.2 Research Objectives .......................................................................... 6

1.3 Organization of Dissertation ............................................................... 7

2. PROBABILISTIC CAPACITY MODEL ……................................................ 10

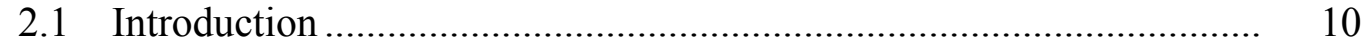

2.2 Probabilistic capacity models........................................................... 12

2.2.1 Review of probabilistic capacity models for pristine bridges .... 12

2.2.2 Proposed probabilistic capacity models for deteriorating

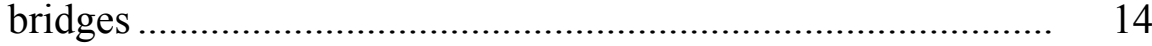

2.3 Flexural stiffness estimation using nondestructive testing.................... $\quad 20$

2.3.1 Damage index method.............................................................. 21

2.3.2 Measurement and model errors ................................................. 24

2.4 Assessment of structural component fragility ..................................... 25

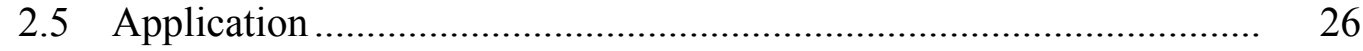

2.6 Conclusions ................................................................................. 31

3. PROBABILISTIC DEMAND MODEL ……………..................................... 34

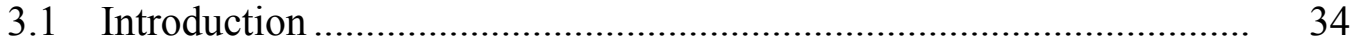

3.2 Virtual experiment demand data ....................................................... 37

3.2.1 Latin hypercube sampling ...................................................... 37

3.2.2 Ground motions...................................................................... 39 
3.2.2 Finite element models .......................................................... 49

3.3 Formulation of probabilistic demand models....................................... 51

3.4 Model assessment............................................................................. 55

3.4.1 Discussion of results.............................................................. 66

3.5 Application of the demand models to estimate seismic fragilities....... $\quad 66$

3.6 Conclusions .............................................................................. $\quad 72$

4. GLOBAL NONDESTRUCTIVE TESTING ……..............................................

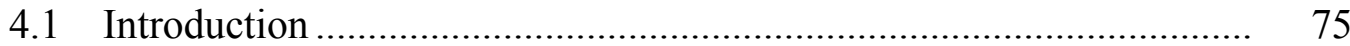

4.2 Proposed damage detection approach .............................................. $\quad 78$

4.2.1 Vibration test .................................................................... 80

4.2.2 Time domain decomposition .................................................. 81

4.2.3 Preliminary FEM............................................................... 83

4.2.4 Bayesian model updating ...................................................... $\quad 83$

4.2.4.1 Markov chain Monte Carlo (MCMC) …………........... 85

4.2.5 Damage index method............................................................. 93

4.3 Uncertainties in the proposed damage detection approach ................... 93

4.4 Propagation of uncertainties............................................................... 97

4.4.1 Measurement errors in ambient vibration test........................... 99

4.4.2 Modeling errors due to TDD ................................................. 102

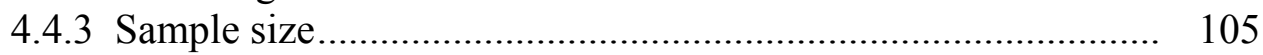

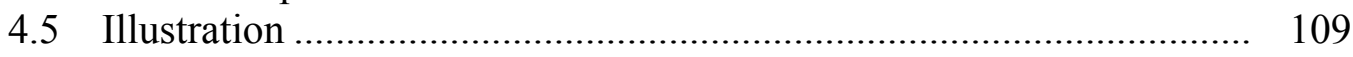

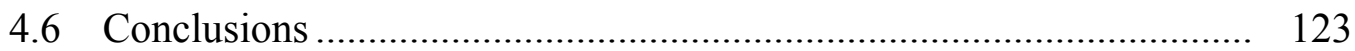

5. LOCAL NONDESTRUCTIVE TESTING ..................................................... 126

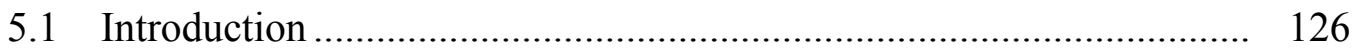

5.2 Review of UPV and rebound hammer tests ..................................... 129

5.3 Development of multivariable linear regression model ....................... 131

5.3.1 Formulation of proposed regression model............................... 131

5.3.2 Data collection...................................................................... 132

5.3.3 Missing data ........................................................................ 133

5.3.4 Assessment of proposed regression model................................ 136

5.4 Evaluation of proposed regression model .......................................... 143

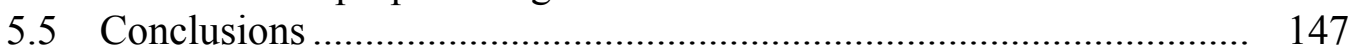

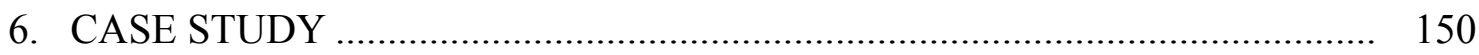

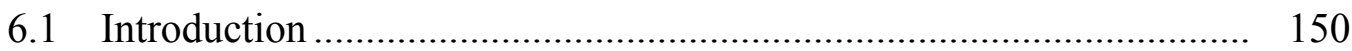

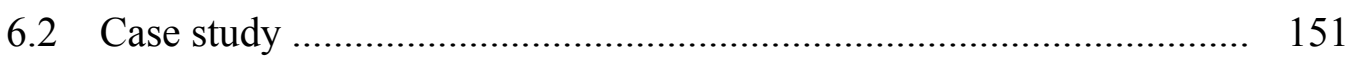

6.2.1 Introduction of the numerical bridge ......................................... 151 
6.2.2 Applying global NDT ....................................................... 154

6.2.2.1 Conduct a vibration test on the damaged FEM 154

6.2.2.2 Determine measurement error and modeling error in TDD ................................................................... 156

6.2.2.3 Identify baseline using Bayesian model updating......... 157

6.2.2.4 Identify damage location by DIM .............................. 160

6.2.3 Applying local NDT ................................................... 162

6.2.4 Assessing fragilities....................................................... 163

6.2.4.1 Importance measure ............................................ 163

6.2.4.2 Fragility estimates .................................................. 166

6.3 Conclusions ........................................................................ 170

7. CONCLUSIONS AND FUTURE WORK .............................................. 172

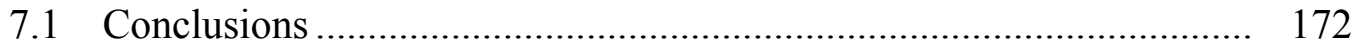

7.2 Future work ....................................................................... 173

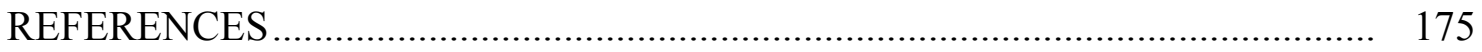

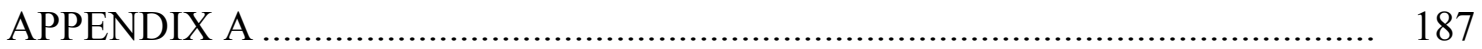

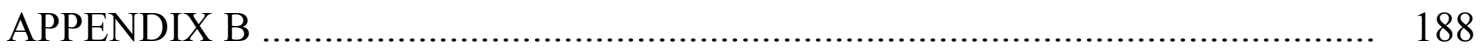

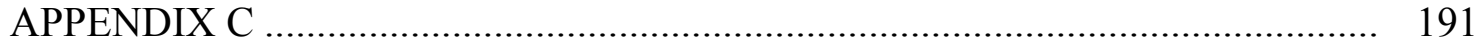

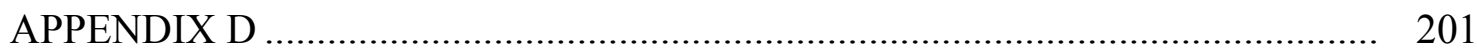

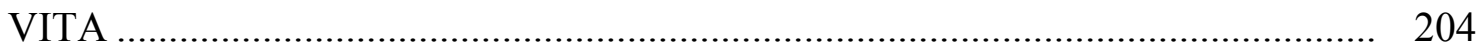




\section{LIST OF FIGURES}

Page

Figure 1-1 Effect of updating input on capacity and demand models

Figure 1-2 Qualitative comparison between the proposed reliability model and a model including corrosion by Choe et al. (2008)

Figure 2-1 Equivalent and local flexural stiffness for a single-column bridge bent

Figure 2-2 The components of yield displacement for a RC bridge column

Figure 2-3 Curvatures along the height of the column

Figure 2-4 Fragility estimate with approximate confidence bounds for the deformation failure of the example column, pre- (thin lines) and post-earthquake (bold lines)

Figure 2-5 Fragility estimate with approximate confidence bounds for the shear failure of the example column, pre- (thin lines) and postearthquake (bold lines)

Figure 2-6 Contour plot of predictive deformation-shear fragility surface of the example column, pre- (thin lines) and post-earthquake (bold lines)

Figure 3-1 Typical one-bent column RC highway bridge configuration

Figure 3-2 Median $\operatorname{PSA}\left(T_{1}\right)$ spectra for each bin with 5\% damping at rock and shallow soil site

Figure 3-3 Median PSA(T $\left.T_{1}\right)$ spectra for each bin with 5\% damping at deep soil site.

Figure 3-4 Comparison of standard deviation of $P S A\left(T_{1}\right)$ values obtained by 20 ground motions (dots) and standard deviation values based on attenuation law by Abrahamson and Silva (1997) (solid lines) for each bin with 5\% damping at rock and shallow soil site.

Figure 3-5 Comparison of standard deviation of $P S A\left(T_{1}\right)$ values obtained by 
20 ground motions (dots) and standard deviation values based on attenuation law by Abrahamson and Silva (1997) (solid lines) for each bin with $5 \%$ damping at deep soil site

Figure 3-6 Comparison between (logarithmic) drift ratio demand predictions based on FEMs (left) and predicted values from probabilistic demand models (right)

Figure 3-7 Comparison between (logarithmic) normalized shear demand predictions based on FEMs (left) and predicted values from probabilistic demand models (right)

Figure 3-8 Univariate fragilities for the example bridge with estimates $E 1_{k}$ (solid curve), $E 2_{k}$ (dotted curve), $E 3_{k}$ (dashed curve), $E 4_{k}$ (dot-dashed curve)

Figure 3-9 Bivariate deformation-shear fragility estimate. 72

Figure 4-1 Damage detection using vibration-based NDT 76

Figure 4-2 Flowchart of the proposed damage detection approach

Figure 4-3 Conceptual illustration of correct and false detection probabilities when a low threshold (left) and a high threshold (right) are used.....

Figure 4-4 Flowchart of the procedures to propagate the measurement noises to the modal data

Figure 4-5 Flowchart of the procedures to estimate the modeling error in TDD 106

Figure 4-6 Schematic of the example beam and the FEM

Figure 4-7 Mean (a) and standard deviation (b) of modeling error in modal frequencies

Figure 4-8 Z values (solid line: mean, dotted line: mean \pm 1 standard deviation for the beam elements with Damage Case 1 under 1\% noise level.

Figure 4-9 Z values (solid line: mean, dotted line: mean \pm 1 standard 
deviation for the beam elements with Damage Case 1 under $2 \%$

noise level.

Figure 4-10 $Z$ values (solid line: mean, dotted line: mean \pm 11 standard deviation) for the beam elements with Damage Case 1 under 3\% noise level.

Figure 4-11 $Z$ values (solid line: mean, dotted line: mean \pm 1 standard deviation) for the beam elements with Damage Case 1 considering error $e_{2}$

Figure 4-12 Probability of damage detection for Damage Cases 1-4 with 10\% flexural stiffness reduction under $1 \%$ noise level using $\lambda=2.0$.....

Figure 4-13 Probability of damage detection for Damage Case 1 with 10\% flexural stiffness reduction under $1 \%$ noise level

Figure 5-1 Diagnostic plots of the proposed model

Figure 5-2 Prediction of Eq. (5.12) model (left) and Eq. (5.13) model (right) using training data

Figure 5-3 Prediction of M1, M3, M5, and M6 suggested by other researchers vs. the true values using training data

Figure 5-4 Comparison of predictions of compressive strength using different regression models vs. the true values.

Figure 6-1 Flowchart of the proposed fragility estimate using NDT.

Figure 6-2 First five mode shapes for the example bridge

Figure 6-3 $Z$ values (solid line: mean, dotted line: mean \pm 1 1standard deviation) for the bridge column elements with under $1 \%$ measurement error

Figure 6-4 Probability of damage detection for the bridge column elements under $1 \%$ measurement error

Figure 6-5 Deformation fragilities of the target damaged FEM (solid lines), the preliminary FEM (large dashed lines), and the identified damaged FEM (small dashed lines) 
Figure 6-6 Shear fragility of the target damaged FEM (solid lines), the preliminary FEM (large dashed lines), , and the identified damaged FEM (small dashed lines) ............................................................

Figure 6-7 Bi-variate fragility of the target damaged FEM (solid lines), the preliminary FEM (large dashed lines), , and the identified damaged FEM (small dashed lines) ................................................................. 


\section{LIST OF TABLES}

Page

Table 2-1 Flexural stiffness of the example column, EI $\left(10^{6} \cdot \mathrm{kN} \cdot \mathrm{m}^{2}\right)$

Table 2-2 Distribution, mean and coefficient of variation (COV) for the random variables in the model

Table 2-3 Mean deformation and shear capacity pre- and post-earthquake......

Table 3-1 Ranges of the design parameters for typical highway bridges with one single-column bent

Table 3-2 Number (percentage) of earthquake records in PEER Strong Motion Database for given GM and USGS soil classification ( Luco, 2002)

Table 3-3 Candidate explanatory functions of normalized intensity measures .

Table 3-4 Comparison of the complexity and accuracy of the developed probabilistic models

Table 3-5 Posterior statistics of the parameters in the deformation model .....

Table 3-6 Posterior statistics of the parameters in the shear model 64

Table 3-7 Posterior statistics of the parameters in the bivariate deformationshear model

Table 3-8 Design parameters for the example bridge.

Table 4-1 Errors and uncertainties in the proposed damage detection approach

Table 4-2 Statistics of model parameters. 113

Table 4-3 Comparison of modal frequencies (Hz)

Table 4-4 Estimates of $e_{3 a}$ for the example beam 114

Table 4-5 Comparison of modal frequencies of no damage case and damage cases 
Table 5-1 Ranges of variables from database

Table 5-2 Posterior statistics of the parameters in the proposed regression model using Group 1 data

Table 5-3 Updated posterior statistics of the parameters in the proposed regression model using Group 2 data

Table 5-4 Posterior statistics of the parameters in the regression model shown in Eq. (5.13) using training data

Table 5-5 A summary of regression formulations developed by different researchers

Table 5-6 A comparison of valid predictive regression models

Table 6-1 Design parameters for the preliminary (identified) FEM of the bridge.

Table 6-2 Parameter ratios between the baseline values and the preliminary values.

Table 6-3 Estimate of modeling errors in modal frequencies

Table 6-4 Statistics of the model parameters

Table 6-5 Comparison of modal frequencies $(\mathrm{Hz})$ 


\section{INTRODUCTION}

\subsection{Background}

Most in-service reinforced concrete (RC) bridges in the US are suffering from aging and deterioration due to harsh environmental exposure conditions and/or are damaged by natural (earthquakes, hydrologic forces, etc.) or man-made (collisions, fire, de-icing salt, etc.) hazards. Consequently, the structural capacity of an existing bridge is typically less than the structural capacity of a new structure. Even if the deterioration does not lead to the direct failure of a structure, it may weaken the structure, making it more vulnerable to earthquakes and other hazards. The collapse of the Minneapolis bridge on August 1, 2007 awakened the nation's awareness of existing bridges safety issues. At least $\$ 140$ billion has been proposed to make major repairs or upgrades to one of every four U.S. bridges, according to the report by American Association of State Highway and Transportation Officials (2008).

With limited funds available for the maintenance of aging and degrading bridges, knowing the ability of RC bridges to withstand future seismic demands during their lifecycle can help bridge owners make rational decisions regarding optimal allocation of resources for maintenance, repair, and/or rehabilitation of bridge systems (Frangopol et al. 2001). To obtain accurate estimates of the residual reliability of a deteriorating bridge, it is reorganized to be important to accurately estimate the actual properties of

This dissertation follows the format of the ASCE Journal of Engineering Mechanics. 
the bridge and properly account for all prevailing uncertainties, including the randomness inherent in the loads, the uncertainties in the material properties, and the uncertainties in the characteristics (time, rate, and location) of the deterioration processes.

Current assessment of the safety of RC bridges is primarily performed by visual inspections. However, visual inspections have several limitations.

- The information provided is subjective and limited. The quantity of visual inspection heavily relies on the knowledge and experiences of the inspector.

- Visual inspections typically detect only very advanced deteriorated conditions and are not able to detect damages at the locations that the inspector cannot reach.

- With the rapid increase of the size and complexity of bridges, the conventional visual inspections become less inefficient, more expensive, and time consuming.

- $\quad$ They are schedule-based.

Nondestructive testing (NDT), on the other hand, can be applied during the operation of structures and is an effective way to evaluate up-to-date in-place structural properties and detect damages. It can also serves as a tool for autonomous and continuous detection. More importantly, it can localize and qualify varying degrees of damage and discover damage at early stage. There is consensus on the importance of NDT which can be implemented as a complement to visual inspection.

Methods using vibration measurement to determine structural properties are referred as global/vibration-based NDT. It uses measureable changes in the structural 
dynamic characteristics caused by damage to identify the damage. Comprehensive review of global NDT can be found in (Doebling et al. 1998; Farrar et al. 2001; Fritzen 2005). Modal frequencies and modal shapes are most common used in global NDTs.

Humar et al. (2006) gave a survey of some commonly used algorithms including methods based on the changes in modal frequencies, modal shapes, modal shape curvatures, flexibility matrix, modal strain energy, and etc. However, global NDT requires an undamaged/baseline structure that is usually not available for existing structures. Moreover, for large structures, global NDT is only effective to identify global/equivalent structural properties and detect the possible damage locations. To further determine the local characteristics of structural properties or detect small defects, local NDT is needed.

The efforts to apply local NDT to the civil engineering field have been concentrated in the approaches using 1) acoustic signals, 2) electromagnetism, 3) radiography, 4) fiber optics, 5) radar and radio frequency, 6) optics, and 7) piezoelectric ceramics (Chang and Liu 2003). Different approaches usually focus on specific structural properties (crack length of concrete, bond strength between concrete and reinforcement, etc.) or different characteristics of a material deterioration process (corrosion initiation time, corrosion rate, etc.). For example, ACI Committee 228 (2003) provides a guidance of using NDT methods to predict compressive strength of concrete, including rebound hammer test, ultrasonic pulse velocity (UPV) test, maturity, and castin-place cylinders. Local NDT can be applied to the suspected damaged area that is identified by global NDT. Therefore, a combination of global and local NDTs is usually 
helpful.

This dissertation proposes to develop an adaptive reliability analysis of $\mathrm{RC}$ bridges incorporating the damage detection information obtained from NDT. NDT can be used to identify damages and provides valuable information to evaluate up-to-date, in-place structural properties. Thus, the uncertainties in the properties of a bridge can be reduced using the bridge actual properties evaluated from NDT. Figure 1-1 illustrates this proposed scheme that uses the actual structural properties to update the capacity and demand models, where PDF refers to the probability of density function. Consequently the reliability will be updated. Compared with the conventional reliability analysis incorporating with the deterioration models, the proposed method accounts for all possible causes of deterioration detected by NDT instead of only considering the degrading mechanisms captured by the deterioration models.

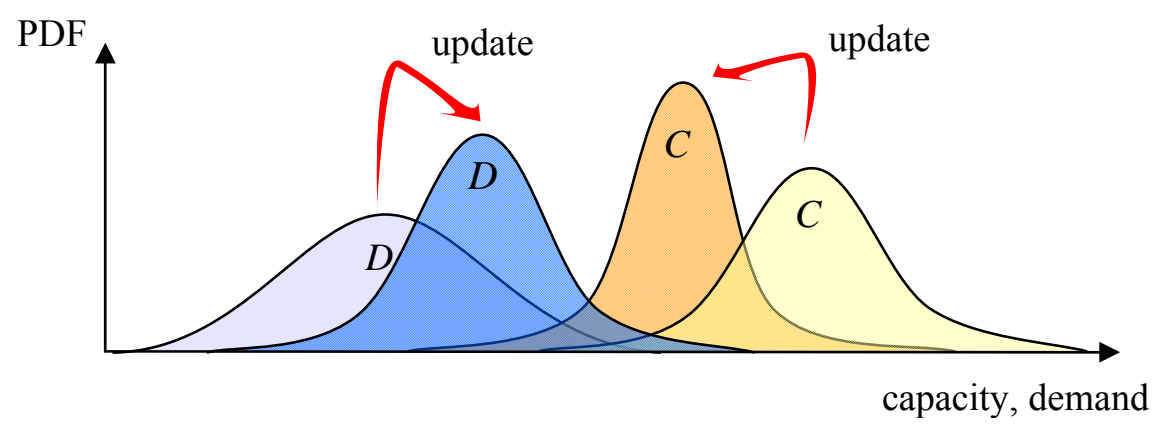

Figure 1-1. Effect of updating input on capacity and demand models

As an illustration of the benefits of the proposed approach, Figure 1-2 shows a qualitative comparison between the proposed reliability model and a traditional time- 
variant reliability model for a RC bridge, e.g., Choe et al. (2008). The proposed adaptive reliability model reduces the uncertainty in the reliability estimate because a damage detection using NDT provides information about the actual properties and conditions of the bridge. It also properly captures the damage caused, for example, by an earthquake that might occur at a certain time $t_{1}$. As shown in Figure 1-2, the earthquake might suddenly damage the bridge, producing a negative impact on reliability, $\Delta P_{f 1}$. Similarly, rehabilitation action might follow the same earthquake leading to an improvement in reliability, $\Delta P_{f_{2}}$. As another example, at time $t_{2}$ the bridge might undergo maintenance that would undo part of the damage due to aging. The proposed adaptive reliability model can incorporate events such as these, providing a more accurate reliability assessment.

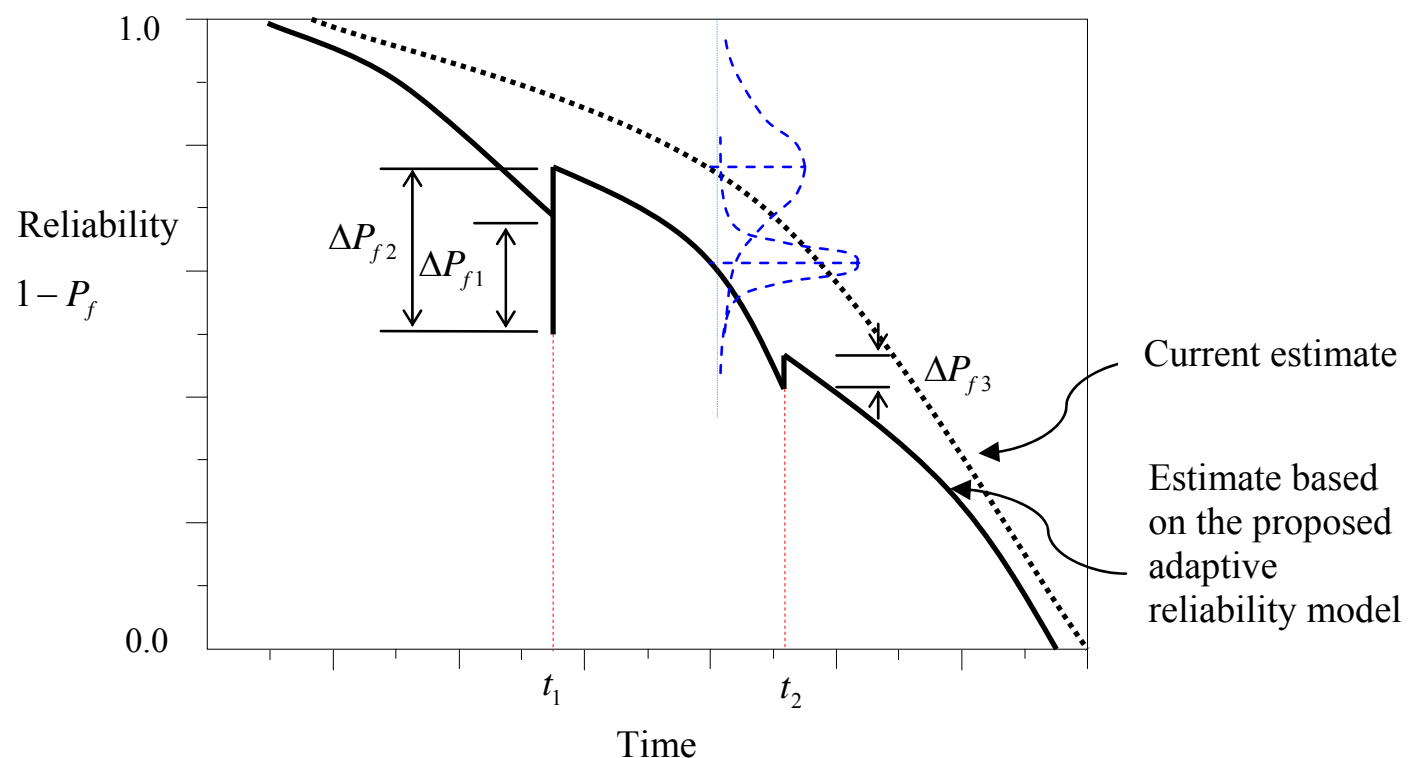

Figure 1-2. Qualitative comparison between the proposed reliability model and a model including corrosion by Choe et al. (2008) 


\subsection{Research Objectives}

The goal of this dissertation is to develop an adaptive reliability framework for $\mathrm{RC}$ bridges that accounts for the information provided by a damage-detection strategy referred to as NDT to determine the current and better predict the future health state of instrumented $\mathrm{RC}$ bridges. The reliability models will be continuously updated by the data obtained from the long-term and continuous damage detection. The proposed work has the following four objectives:

Objective 1: Develop probabilistic deformation and shear capacity models for $R C$ bridge columns. Since the failure of bridge columns is vital to the whole structure, the system failure of a RC bridge will be defined as the failure of any column. While capacity models for RC bridge columns have been developed (Gardoni et al. 2002; Choe et al. 2007), the existing models do not account for the degradation in the flexural stiffness that typically varies along the column height due to different exposure conditions and loadings. This objective is to extend the developed capacity models to account for non-uniform degradation in RC columns.

Objective 2: Develop probabilistic seismic deformation and shear demand models for RC bridge with one single-bent column. The proposed demand models will fully consider all the relevant uncertainties associated with the structural demands on $\mathrm{RC}$ bridges due to seismic excitations. Such uncertainties include uncertainties in the ground motions and the structural properties, model errors, and statistical uncertainties in the model parameters. 
Objective 3: Develop probabilistic damage detection using NDT considering measurement error and modeling error. Despite of the extensive study of damage detection methods, it has been increasingly recognized that a considerable amount of errors exist in the measurement data and in the damage detection process. Through developing probabilistic damage detection in this objective, the measurement and modeling error can be incorporated.

Objective 4: Incorporate the data from NDT into the probabilistic capacity and demand models. This objective reflects the ultimate goal of this proposal: to use the information obtained from NDT to evaluate the current and future performance of a structure. Additionally, the evaluation can be continuously updated as new information becomes available.

The work proposed by this dissertation can lead to a significant decrease in lifecycle and maintenance costs for RC bridges based on the accurate estimate of their reliability that can be used for optimal allocation of resources for maintenance, repair, and rehabilitation. Furthermore, the outcomes of the proposed work will be of interest to the international civil engineering community as well.

\subsection{Organization of Dissertation}

This dissertation is organized using a section-subsection format. There are six sections and within each section there are subsections. The word "section" corresponds to the first heading level and "subsection" corresponds to the second, third, and fourth heading levels. Following are brief descriptions for each section in this dissertation. 
- Section 1 (current section) gives an introduction about the background of this dissertation, including the problem statement, the current available solutions, and the proposed ideas. Then, the objectives and the structure of this dissertation are given.

- Section 2 develops deformation and shear capacity models for RC bridge columns that incorporate information obtained from NDT. The proposed models can be used when the flexural stiffness decays non-uniformly over a column height. The flexural stiffness of a column is estimated based on measured acceleration responses using a system identification method and the damage index method. This work has been published in the Journal of Engineering Mechanics ASCE, 135 (12) with the title of "Probabilistic Capacity Models and Fragility Estimates for Reinforced Concrete Columns Incorporating NDT Data”.

- Section 3 presents a general methodology to construct probabilistic demand models for RC highway bridges with one single-column bent. The developed probabilistic models consider the dependence of the seismic demands on the ground motion characteristics and the prevailing uncertainties, including uncertainties in the structural properties, statistical uncertainties, and model errors. This work has been summarized in a Journal paper titled "Probabilistic Seismic Demand Models and Fragility Estimates for Reinforced Concrete Highway Bridges with One Single-Column Bent" and submitted to Journal of Engineering Mechanics ASCE. 
- Section 4 proposes a novel probabilistic vibration-based damage detection approach that accounts for the underlying uncertainties. This vibration-based damage detection serves as a global NDT in this dissertation. In particular, the proposed approach considers the measurement errors in the vibration tests, the modeling errors in the damage detection process, and the statistical uncertainties in the unknown model parameters. This work is summarized into two Journal papers: one is titled with "Extracting Modal Parameters Considering Measurement and Modeling Errors" and has been submitted to Journal of Risk and Reliability, and the other one is titled with "A Probabilistic Damage Detection Approach Using Vibration-based Nondestructive Testing" and has been submitted to Structural Safety.

- Section 5 develops a probabilistic multivariable linear regression model to predict the compressive strength of concrete using a combination of rebound hammer and ultrasonic pulse velocity tests, two local NDT test. This work has been summarized in a Journal paper titled "Predicting Concrete Compressive Strength Using Ultrasonic Pulse Velocity and Rebound Number Data" and submitted to ACI Materials Journal.

- Section 6 applies the overall framework proposed in this dissertation to a $\mathrm{RC}$ bridge with a one-column bent. Following with this illustration, it is the conclusions of this dissertation. 


\section{PROBABILISTIC CAPACITY MODEL}

\subsection{Introduction}

When a capacity model is used to estimate the residual reliability of a deteriorating bridge, it is essential to incorporate the actual properties of the structure. Previous work either only assessed the reliability of bridges without considering structural deterioration, underestimating the actual vulnerability of bridges, or used deterministic or probabilistic deterioration models to account for the deterioration. For example, Gardoni et al. (2002, 2003) and Choe et al. (2007) assessed the reliability of pristine bridges by developing probabilistic capacity models for circular RC bridge columns without considering the structural deterioration. Val et al. (1997), Enright and Frangopol (1998), Stewart and Rosowsky (1998), Vu and Steward (2000), and Choe et al. (2008) used probabilistic corrosion models to account for the corrosion initiation time and the corrosion propagation rate. Fajfar and Gašperšič (1996), Williams and Sexsmith (1997), van de Lint and Goh (2004), Teran-Gilmore and Bahena-Arredonom (2008), and Kumar et al. (2009) used mathematical models to capture the effects of cumulative seismic damage. However, the deterioration models are derived based on laboratory data or from the behavior of other similar structures under similar conditions (Estes et al. 2003). As a result, additional uncertainties associated with the deterioration models are introduced into the reliability analysis. Furthermore, deterioration models are limited to specific deterioration mechanisms. Finally, whether or not the deterioration process has been accounted for, the basic material properties and parameters used in the capacity model 
are generally assumed using typical values and are not customized for a particular structure, leading to a significant degree of inaccuracy.

In this Section, the deformation and shear capacity models previously developed by Gardoni et al. (2002) and Choe et al. (2007) for RC circular columns are extended to incorporate field measured information about the flexural stiffness, which is typically non-uniform along a column height. By avoiding using deterioration models, the proposed formulation has two main advantages over the previous approaches 1) actual values for the material properties are used instead of assumed values; and 2) the approach accounts for all possible causes of deterioration instead of only the mechanisms captured by the deterioration models.

In a case study, the developed capacity models are used to estimate the fragility (or the conditional probability of attaining or exceeding the capacity level) of the column in the Lavic Overcrossing Bridge for a given deformation or shear demand. In 1999, this two-span concrete box-girder bridge located in Southern California was subject to the Hector Mine Earthquake. In this section, the pre- and post-earthquake estimates of the univariate shear and deformation fragilities and the bivariate shear-deformation fragility are computed and compared. To estimate the flexural stiffness of a column, the system identification method and the damage index method developed by Stubbs et al. (1996) based on the bridge eigenmodes are used in this study. NDT is used to record the bridge acceleration responses and extract the eigenmodes. Both displacement and shear capacities are found to decrease after the Hector Mine Earthquake. Furthermore, the result shows that the damage due to the earthquake has more impact on the shear 
capacity than the deformation capacity, leading to a more significant increment in the shear fragility than on the deformation fragility. While the work in this study focuses on incorporating the information about the flexural stiffness changes of the column, the same methodology can be extended to account for other information on structural conditions that might be obtained from NDT.

In the following, the probabilistic capacity models developed by Gardoni et al. (2002) and Choe et al. (2007) are briefly reviewed. These models are then extended to account for the field data from NDT. Next, how the flexural stiffness of RC columns can be obtained using NDT techniques and how measurement and model errors can be accounted for are described. Then, an assessment of the structural fragility of RC columns that accounts for the present uncertainties is described. Finally, as an application of the proposed approach, the fragilities of the Lavic Overcrossing bridge column are assessed based on the estimated flexural stiffness before and after the Hector Mine Earthquake.

\subsection{Probabilistic Capacity Models}

\subsubsection{Review of Probabilistic Capacity Models for Pristine Bridges}

Gardoni et al. (2002) and Choe et al. (2007) developed probabilistic capacity models for circular RC bridge columns in a pristine state without considering the effects of deterioration. These models account for model errors that arise from potential inaccuracies in the model form and missing variables, and statistical uncertainties. The probabilistic deformation and shear capacity models are expressed as 


$$
C_{k}\left(\mathbf{x}, \boldsymbol{\Theta}_{C, k}\right)=\hat{c}_{k}(\mathbf{x})+\gamma_{C, k}\left(\mathbf{x}, \boldsymbol{\theta}_{C, k}\right)+\sigma_{C, k} \varepsilon_{C, k} \quad k=\delta, v
$$

where $\hat{c}_{k}(\mathbf{x})=$ selected deterministic capacity model, $\gamma_{C, k}\left(\mathbf{x}, \boldsymbol{\theta}_{C, k}\right)=$ correction term for the bias inherent in the deterministic model, $\boldsymbol{\Theta}_{C, k}=\left(\boldsymbol{\theta}_{C, k}, \sigma_{C, k}\right)=$ a vector of unknown model parameters, $\sigma_{C, k}=$ standard deviation of the model error, $\varepsilon_{C, k}=$ random variable with zero mean and unit variance, $\mathbf{x}=\mathrm{a}$ vector of basic variables, e.g., material properties, structural dimensions, and imposed boundary conditions, as per build-in state. The index $k$ indicates the mode of failure considered, i.e., deformation failure $(k=\delta)$ or shear force failure $(k=v)$. The unknown parameters in the models are $\boldsymbol{\Theta}_{C, k}=\left(\boldsymbol{\Theta}_{C, \delta}, \boldsymbol{\Theta}_{C, v}, \rho_{C, \delta v}\right)$, where $\rho_{C, \delta v}=$ correlation coefficient between the model errors $\sigma_{C, \delta} \varepsilon_{C, \delta}$ and $\sigma_{C, v} \varepsilon_{C, v}$. In developing the models in Eq. (2.1), Gardoni et al. (2002) and Choe et al. (2007) used a logarithmic transformation of the deformation and shear capacity to satisfy the homoskedasticity assumption $\left(\sigma_{C, k}\right.$ is independent of $\left.\mathbf{x}\right)$ and the normality assumption ( $\varepsilon_{C, k}$ follows the Normal distribution). These two assumptions are needed to assess the unknown parameters $\boldsymbol{\Theta}_{C, k}$. Additional details on how to obtain $\boldsymbol{\Theta}_{C, k}$ using experimental data can be found in Gardoni et al. (2002).

The selected deterministic model for the deformation capacity $\hat{c}_{\delta}(\mathbf{x})$ consists of two terms: an elastic component, $\hat{\Delta}_{y}$, and a plastic component, $\hat{\Delta}_{p}$, computed assuming a bilinear approximation for the column moment-curvature relation (Priestley et al. 1996). The elastic component $\hat{\Delta}_{y}$ can be further partitioned into three components: a 
flexure component $\hat{\Delta}_{f}$ based on a linear curvature distribution along the column height, a shear component $\hat{\Delta}_{s h}$ due to the shear distortion, and a slip component $\hat{\Delta}_{s l}$ due to the slipping of the longitudinal bar reinforcement at the base. For the shear capacity, the selected deterministic model $\hat{c}_{v}(\mathbf{x})$ is the sum of contribution of the transverse steel, $\hat{V}_{s}$, and the contribution of the concrete, $\hat{V}_{c}$.

\subsubsection{Proposed Probabilistic Capacity Models for Deteriorating Bridges}

The models developed by Gardoni et al. (2002) and Choe et al. (2007) do not account for the degradation in the flexural stiffness that typically varies along the column height due to different exposure conditions and loadings. Here the capacity models described above are extended to account for non-uniform degradation in RC columns.

To develop the deformation capacity model for a column with non-uniform stiffness, a column with clear height $H$ is divided into $n$ finite segments (Figure 2-1) such that each segment $i(i=1,2, \ldots, n)$ located at a distance $h_{i}$ from the base of the column has approximately uniform flexural stiffness $\left.(E I)_{i}\right|_{t}$ over its height $\Delta h_{i}$ at time $t$. The time $t$ indicates when the measured data are recorded in the field. If a lateral force, $F_{i}$, is applied to the top of the column such that the section in the $i$ th segment reaches the yield curvature, $\phi_{y, i}$, the relation between $F_{i}$ and $\phi_{y, i}$ can be found as $F_{i}=\phi_{y, i}(E I)_{i} / \Delta h_{i}$. The critical cross section is defined as the one that reaches yielding first and the corresponding yielding force $F_{y}$ is defined as $F_{y}=\min _{i=1, \ldots, n}\left(F_{i}\right)$. If it is 
assumed that there is only one critical cross section and it is located in the qth segment, then the lateral force making the column cross section yield is $F_{y}=F_{q}$. In the case of uniform flexural stiffness, the critical section is always at the bottom of a column where the moment is largest. In the case considered here of non-uniform flexural stiffness, the critical section might not be at the bottom of the column.

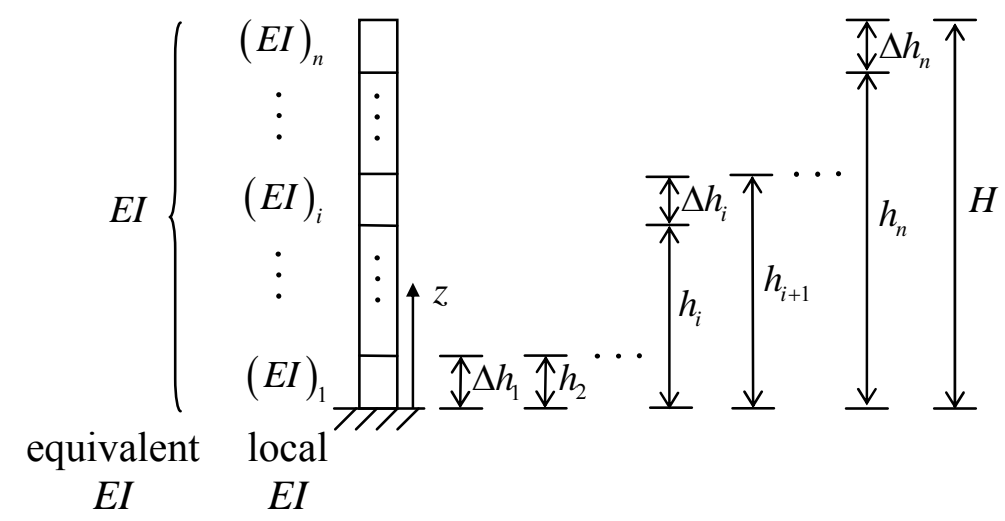

Figure 2-1. Equivalent and local flexural stiffness for a single-column bridge bent

In the case of deterioration over time, the vector $\mathbf{x}$ in Eq. (2.1) can be partitioned as $\mathbf{x}=\left[\mathbf{x}_{1},\left.\mathbf{x}_{2}\right|_{t}\right]$ where $\mathbf{x}_{1}=\mathrm{a}$ vector of time-invariant variables and $\left.\mathbf{x}_{2}\right|_{t}=$ a vector of time-variant variables. Since the flexural stiffness is the focus of this study, $\left.\mathbf{x}_{2}\right|_{t}$ refer to those variables related to the flexural stiffness.

Using the segmented column, $\left.\hat{\Delta}_{f}\right|_{t}$ can be obtained as $\left.\hat{\Delta}_{f}\right|_{t}=\left.u(z=H)\right|_{t}$, where $u(z)=$ flexural displacement along the column height that is the solution of the following differential equation: 


$$
\frac{\left.\mathrm{d}^{2} u(z)\right|_{t}}{\mathrm{~d} z^{2}}=\frac{\left.M(z)\right|_{t}}{\left.E I(z)\right|_{t}}
$$

where $\left.M(z)\right|_{t}=\left.\left.F\right|_{t} l_{\text {eff }}\right|_{t}\left[1-z /\left.l_{\text {eff }}\right|_{t}\right]=$ moment at height $z$, in which $\left.l_{\text {eff }}\right|_{t}=H+\left.Y P\right|_{t}$ if $q=1$ (i.e., the critical section is at the base of the column) and $\left.l_{\text {eff }}\right|_{t}=H$ if $q \neq 1$, where $Y P$ is the depth of the yield penetration into the column base. Based on analysis and test results (Priestley et al. 1996), YP $\left.\right|_{t}=\left.0.022 f_{y}\right|_{t} d_{b}$ where $\left.f_{y}\right|_{t}=$ yield strength of the longitudinal reinforcement (in $\mathrm{MPa}$ ) and $d_{b}=$ diameter of the longitudinal reinforcement. The flexural stiffness $\left.E I(z)\right|_{t}$ is piece-wise uniform with value $\left.(E I)_{i}\right|_{t}$ in each segment $i$ at time $t$.

Similarly, $\hat{\Delta}_{s h}$ can be obtained accounting for the time dependency of the angle of rotation $\left.\varphi_{i}\right|_{t}$ of each segment due to $F_{y}$. The angle of rotation can be computed as $\left.\varphi_{i}\right|_{t}=\tan ^{-1}\left[F_{y} /\left(\left.\left.G_{i}\right|_{t} \cdot A_{v e, i}\right|_{t}\right)\right]$, where $\left.A_{v e, i}\right|_{t}=\left.k_{I, i}\right|_{t} k_{s} A_{g}=$ effective shear area for the $i$ th segment, in which $k_{s}=0.9$ represents the shape factor for a circular cross section, $\left.k_{I, i}\right|_{t}=\left.(E I)_{i}\right|_{t} / E I$ where $E I$ is the pristine flexural stiffness of the column, and $A_{g}=$ gross cross sectional area. Accordingly, $\hat{\Delta}_{\text {sh }}$ can be calculated as

$$
\left.\hat{\Delta}_{s h}\right|_{t}=\sum_{i=1}^{n}\left(\left.\varphi_{i}\right|_{t} \cdot \Delta h_{i}\right)
$$

The contribution $\left.\hat{\Delta}_{s l}\right|_{t}$ is present only when the critical section is at the base of the column $(q=1)$ since the local rotation at the base does not need to be accounted for when the base section remains elastic (Alsiwat and Saatcioglu 1992). When the critical 
section is not at the base of the column $(q \neq 1),\left.\hat{\Delta}_{s l}\right|_{t}$ is equal to zero. Thus, $\left.\hat{\Delta}_{s l}\right|_{t}$ can be written as

$$
\left.\hat{\Delta}_{s l}\right|_{t}= \begin{cases}\frac{\left.\left.\varphi_{y, m}\right|_{t} \cdot f_{y}\right|_{t} \cdot d_{b} H}{\left.8 \mu\right|_{t}} & q=1 \\ 0 & q \neq 1\end{cases}
$$

where $\left.\mu\right|_{t}=1.08 \sqrt{\left.f_{c}^{\prime}\right|_{t}}$, and $f_{c}^{\prime}=$ concrete compressive strength (in MPa). The equation for $q=1$ was developed by Pujol et al. (1999). Figure 2-2 shows the three contributions to $\hat{\Delta}_{y}$ in case of non-uniform flexural stiffness.
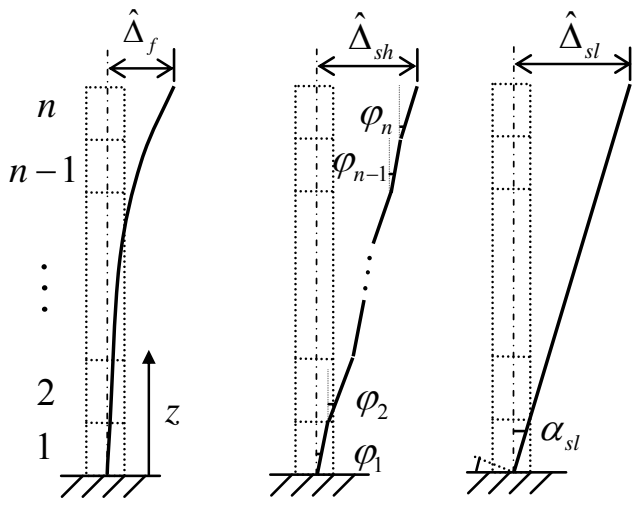

Figure 2-2. The components of yield displacement for a RC bridge column

Considering the tension cracking of the concrete along with the yielding of the longitudinal reinforcement, the plastic curvature is assumed to follow an equivalent trapezoidal shape as shown in Figure 2-3 (Priestley et al. 1996). The plastic deformation, $\left.\hat{\Delta}_{p}\right|_{t}$, can then be written as 


$$
\left.\hat{\Delta}_{p}\right|_{t}=\left.\left.\phi_{p, q}\right|_{t} \cdot l_{p}\right|_{t} \cdot\left(H-\sum_{i=1}^{q-1} h_{i}\right)
$$

where $\left.\phi_{p, q}\right|_{t}=\left.\phi_{u, q}\right|_{t}-\left.\phi_{y, q}\right|_{t}=$ plastic curvature, $\left.\phi_{u, q}\right|_{t}=$ ultimate curvature for the critical cross section, $l_{p}=$ plastic hinge length calculated based on analysis and test results (Priestley et al. 1996) as $\left(0.08 H+\left.0.022 f_{y}\right|_{t} d_{b}\right) \geq\left. 0.044 f_{y}\right|_{t} d_{b}$ with $f_{y}$ in MPa units.
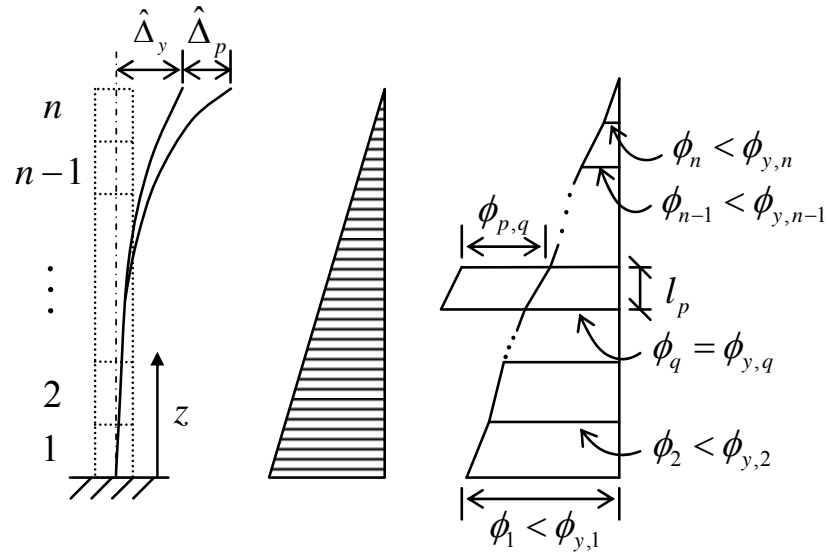

Deformation Moment Curvature

Figure 2-3. Curvatures along the height of the column

Following Gardoni et al. (2002) and Choe et al. (2007), $\gamma_{\delta}\left[\mathbf{x}_{1},\left.\mathbf{x}_{2}\right|_{t}, \boldsymbol{\theta}_{C, \delta}\right]$ can be

written as

$$
\gamma_{\delta}\left[\mathbf{x}_{1},\left.\mathbf{x}_{2}\right|_{t}, \boldsymbol{\theta}_{C, \delta}\right]=\theta_{C, \delta 1}+\theta_{C, \delta 2} \frac{\left.4 V_{I, q}\right|_{t}}{\left.\pi D_{g}^{2} f^{\prime}\right|_{t}}+\left(-0.099 \theta_{C, \delta 3}-0.7614\right) \frac{\left.\rho_{s} f_{y h}\right|_{t} D_{c}}{\left.f_{c}^{\prime}\right|_{t} D_{g}}+\left.\theta_{C, \delta 3} \varepsilon_{c u}\right|_{t}
$$

where the posterior statistics of the model parameters $\boldsymbol{\theta}_{C, \delta}=\left(\theta_{C, \delta 1}, \theta_{C, \delta 2}, \theta_{C, \delta 3}\right)$ are provided in Appendix A, $\left.V_{I, q}\right|_{t}=\left.M_{I, q}\right|_{t} / H$ in which $\left.M_{I, q}\right|_{t}$ denotes the ideal 
(theoretical) moment capacity corresponding to the idealized yield curvature $\left.\phi_{y, q}\right|_{t}$, $D_{g}=$ gross (or outer) column diameter, $\left.f_{t}^{\prime}\right|_{t}=0.5 \sqrt{\left.f_{c}^{\prime}\right|_{t}}=$ tensile strength of concrete with $\left.f_{c}^{\prime}\right|_{t}$ in MPa, $D_{c}=$ core column diameter (defined as the diameter of the concrete contained within the centerline of the spiral reinforcement), $\left.f_{y h}\right|_{t}=$ yield stress of the transverse reinforcement, $\rho_{s}=$ volumetric transverse reinforcement ratio, and $\left.\varepsilon_{c u}\right|_{t}=$ ultimate confined concrete compressive strain.

For the shear capacity mode, the contribution $\hat{V}_{s}$ is written as

$$
\left.\hat{V}_{s}\right|_{t}=\frac{\left.A_{v} f_{y h}\right|_{t} D_{e}}{S}
$$

where $A_{v}=2 A_{h}=$ total area in a layer of the transverse reinforcement in the direction of the shear force, in which $A_{h}=$ cross-sectional area at the transverse reinforcement, $D_{e}=0.8 D_{g}=$ effective depth for circular cross section, and $S=$ spacing of transverse reinforcements. The contribution $\left.\hat{V}_{c}\right|_{t}$ is computed based on the model developed by Moehle et al. (1999, 2000) as

$$
\left.\hat{V}_{c}\right|_{t}=\left.R\right|_{t}\left[\frac{\left.f_{t}^{\prime}\right|_{t}}{a / D_{e}} \sqrt{1+\frac{N}{\left.f_{t}^{\prime}\right|_{t} A_{g}}}\right]
$$

where $\left.R\right|_{t}$ is a factor that accounts for the strength degradation within the plastic hinge region and is a function of the displacement ductility, and $a / D_{e}=$ aspect ratio, in which $a=$ distance from the location of the maximum moment to the inflection point. Note that one could use other models for $\hat{V}_{s}$ and $\hat{V}_{c}$ as suggested in different literature (e.g., 
ASCE-ACI Joint Task Committee 426 1973; ATC-32 1996; Kowalsky and Priestley 2000; Kim and Mander 2007), and then follow the procedures suggested by Gardoni 2003 to develop the corresponding probabilistic capacity models.

The correction term $\gamma_{v}\left[\mathbf{x}_{1},\left.\mathbf{x}_{2}\right|_{t}, \boldsymbol{\theta}_{C, v}\right]$ for the shear capacity model in this study is written as

$$
\gamma_{v}\left[\mathbf{x}_{1},\left.\mathbf{x}_{2}\right|_{t}, \boldsymbol{\theta}_{C, v}\right]=\theta_{C, v 1} \rho_{l}+\theta_{C, v 2} \frac{\left.A_{v} f_{y h}\right|_{t} D_{g}}{\left.A_{g} f_{t}^{\prime}\right|_{t} S}
$$

where $\rho_{l}=$ longitudinal reinforcement ratio and the posterior statistics of the model parameters $\boldsymbol{\theta}_{C, v}=\left(\theta_{C, v 1}, \theta_{C, v 2}\right)$ are given in Appendix A. In addition, Appendix A provides the posterior statistics for $\boldsymbol{\Theta}_{C}=\left(\boldsymbol{\Theta}_{C, \delta}, \boldsymbol{\Theta}_{C, v}, \rho_{C, \delta v}\right)$ that are needed to define the bivariate deformation-shear capacity model.

\subsection{Flexural Stiffness Estimation Using Nondestructive Testing}

This study proposes to assess the deteriorated flexural stiffness of a column using vibration-based NDT. The vibration-based NDT is an emerging technique based on the principle that the occurrence of deterioration alters the structural dynamic characteristics (e.g., eigenmodes) of a bridge (Hurlebaus and Gaul 2006). Thus, measuring and evaluating the dynamic characteristics can aid in detecting the location and severity of the deterioration. There are several advantages of adopting vibration-based NDT (Humar et al. 2006): first, prior knowledge about the locations of the deterioration in the structure is not required; second, the sensors (e.g. accelerometers) used to measure the 
dynamic responses do not need to be put in the vicinity of the deterioration; and last, a limited number of sensors is enough to obtain sufficient information needed to detect the deterioration in a large and complex structure such as a bridge.

The dynamic responses of a bridge can be recorded either by exciting the bridge using a drop weight impact hammer or by considering ambient excitations such as wind loading, traffic loading, etc. If the input is known, conventional modal analysis techniques can be applied to extract the eigenmodes. If the input is unknown, outputonly methods have to be used. Examples of these methods are the frequency domain decomposition that requires a singular value decomposition technique (Brinker et al. 2001) and the time domain decomposition that provides a more accurate estimation of mode shapes (Kim et al. 2005). After extracting the eigenmodes, the system identification and the damage index method proposed by Stubbs et al. (1996) can be used to estimate the stiffness change of the bridge.

\subsubsection{Damage Index Method}

In order to use the damage index method to estimate the flexural stiffness at time $t+\Delta t$, the bridge stiffness at time $t$ and the change in the modal shapes over a time interval $\Delta t$ are needed. However, in general, only the properties of the bridge at $t+\Delta t$ are known and the stiffness and modal shapes at $t$ are not available. To overcome this problem, Stubbs et al. (1996) proposed to developed a reference bridge that replaces the bridge at time $t$ using a finite element model that has the same modal frequencies as the bridge at $t+\Delta t$ but no damage (i.e., each component has uniform stiffness over its length or 
height). Therefore the reference bridge has different modal shapes. A reference bridge is generated by adjusting the stiffness or mass of the structural components in the finite element model.

The damage index method assumes that the ratio between the modal energy of the $i$ th segment, $S_{j i}$, and the modal energy of a whole component in the $j$ th eigenmode, $S_{j}$, remains approximately the same over time, i.e. $\left.S_{j i}\right|_{t} /\left.\left.S_{j}\right|_{t} \approx S_{j i}\right|_{t+\Delta t} /\left.S_{j}\right|_{t+\Delta t}$. Kim and Stubbs (2002) have shown that this assumption is a good approximation when $S_{j} \gg S_{j i}$, which is obtained when a component is divided into a sufficient number of segments. If a column with height $H$ of either the bridge at $t$ or of the reference bridge can be considered as a Bernoulli-Euler beam, the strain energy for the whole column and ith segment can be found as $\left.S_{j}\right|_{t}=\left.\int_{0}^{H} E I(z)\right|_{t}\left[\left.\varphi_{j}^{\prime \prime}(z)\right|_{t}\right]^{2} \mathrm{~d} z \quad$ and $\left.\quad S_{j i}\right|_{t}=\left.(E I)_{i}\right|_{t} \int_{h_{i}}^{h_{i}+\Delta h_{i}}\left[\left.\varphi_{j}^{\prime \prime}(z)\right|_{t}\right]^{2} \mathrm{~d} z \quad$ respectively, where $\left.E I(z)\right|_{t}$ is the piece-wise uniform stiffness of the ith segment with value $\left.(E I)_{i}\right|_{t}$, $\left.\varphi_{j}^{\prime \prime}(z)\right|_{t}$ denotes the $j$ th mode curvature, $h_{i}$ and $h_{i}+\Delta h_{i}$ are the geometric bounds of the $i$ th segment. It should be note that when a reference bridge is used, each segment $i$ has the same flexural stiffness within a component, $\left.(E I)_{i}\right|_{t}=\left.(E I)\right|_{t}$ for every $i$. Thus, the damage index for the $i$ th segment in the $j$ th mode can be expressed as

$$
D I_{j i}=\frac{\left.(E I)_{i}\right|_{t+\Delta t}}{\left.(E I)_{i}\right|_{t}}=\frac{\left.\int_{0}^{H} E I(z)\right|_{t+\Delta t}\left[\left.\varphi_{j}^{\prime \prime}(z)\right|_{t+\Delta t}\right]^{2} \mathrm{~d} z}{\int_{h_{i}}^{h_{i}+\Delta h_{i}}\left[\left.\varphi_{j}^{\prime \prime}(z)\right|_{t+\Delta t}\right]^{2} \mathrm{~d} z} \cdot \frac{\int_{h_{i}}^{H}\left[\left.\varphi_{j j}^{\prime \prime}(z)\right|_{t}\right]^{2} \mathrm{~d} z}{\left.\int_{0}^{h_{i}+\Delta h_{i}} E I(z)\right|_{t}\left[\left.\varphi_{j}^{\prime \prime}(z)\right|_{t}\right]^{2} \mathrm{~d} z}
$$


The flexural stiffness at time $t+\Delta t,\left.(E I)_{i}\right|_{t+\Delta t}$, can be obtained by multiplying $\left.(E I)_{i}\right|_{t}$ by $D I_{j i}$. If the first $N$ modes are used, the damage index is modified as

$$
D I_{i}=\frac{\left.\sum_{j=1}^{N} f_{j i}\right|_{t+\Delta t}}{\left.\sum_{j=1}^{N} f_{j i}\right|_{t}}
$$

where $\left.f_{j i}\right|_{t}=\left.\int_{h_{i}}^{h_{i}+\Delta h_{i}}\left[\varphi_{j j}^{\prime \prime}(z)\right]\right|_{t} ^{2} \mathrm{~d} z /\left.\left.\int_{0}^{H} E I(z)\right|_{t}\left[\varphi_{j}^{\prime \prime}(z)\right]\right|_{t} ^{2} \mathrm{~d} z$ and a similar expression can be found for $\left.f_{j i}\right|_{t+\Delta t}$. The curvature function $\varphi_{j}^{\prime \prime}(z)$ can be calculated using central difference methods using the mode shape data at the recording points or the values from an interpolation of the mode shapes at the recording points. In particular, to estimate $\varphi_{j}^{\prime \prime}(0)$ and $\varphi_{j}^{\prime \prime}(H)$, the values of $\varphi_{j}(-\Delta h)$ and $\varphi_{j}(H+\Delta h)$ are needed. To estimate them, a cubic-spline interpolation is suggested. The inaccuracy in predicting $\varphi_{j}(-\Delta h)$ and $\varphi_{j}(H+\Delta h)$ may lead to the inaccurate estimates of $D I$ for the boundary segments.

To further generalize the DI independently of the structure type, a normalized $D I_{i}, Z_{i}$, is introduced as,

$$
Z_{i}=\frac{D I_{i}-\mu_{D I}}{\sigma_{D I}}
$$

where $\mu_{D I}$ and $\sigma_{D I}$ refer to the mean and standard deviation of $D I_{i}$. To assess whether damage exists in a specific segment, $Z_{i}$ should be compared with a threshold value, which is discussed in Section 4. Note that the mode shapes are not affected by changes in the environmental parameters, such as temperature and humidity, because the changes 
tend to be uniform over the entire structure and not localized to a specific portion. Therefore, the damage index method, which uses modal shape curvatures, is not sensitive to changes in the environmental parameters.

\subsubsection{Measurement and Model Errors}

In estimating material properties from field data, errors are present in the field measurement of the acceleration, here called measurement error, and in the process of obtaining the material properties from the measured accelerations, here called model error. As discussed previously, the process of obtaining the material properties from the measured accelerations requires first extracting the modal parameters, then identifying the damages using the damage index method, and finally estimating the unknown material properties. Model errors are associated to each step of this process. Model errors can be due to 1) changes in boundary or environment conditions between the reference and the deteriorated structures, 2) the limited number of accelerometers, and 3) simplifications in the data processing and analysis. To account for the measurement and model errors, the variables $\left.\mathbf{x}_{2}\right|_{t}$ are written as

$$
\left.\mathbf{x}_{2}\right|_{t}=\left.\hat{\mathbf{x}}_{2}\right|_{t}+\sigma_{e} \varepsilon_{e}
$$

where $\left.\hat{\mathbf{x}}_{2}\right|_{t}=$ estimated value obtained from NDT, $\varepsilon_{e}=$ a random variable with zero mean and unit variance, and $\sigma_{e}=$ standard deviation of the measurement and model error. The formulation in Eq. (2.13) assumes that any systematic measurement and model error have been corrected by calibration. Furthermore, the value of $\sigma_{e}$ can be assessed based 
on engineering judgment and experience or calibration using more accurate measurements and more refined models.

\subsection{Assessment of Structural Component Fragility}

In this study, the univariate deformation and shear fragilities and the bivariate deformation-shear fragility of RC columns are estimated using the developed capacity models that reflect the actual flexural stiffness. Fragility is defined as the conditional probability of attaining or exceeding a specified capacity given a demand level. Following the conventional notation in structural reliability, the event $\left\{g_{k}\left[\mathbf{x}_{1},\left.\mathbf{x}_{2}\right|_{t}, \boldsymbol{\Theta}_{C, k}\right] \leq 0\right\}$ denotes the failure of a bridge column in the $k$ th failure mode.

The limit state function $g_{k}\left[\mathbf{x}_{1},\left.\mathbf{x}_{2}\right|_{t}, \boldsymbol{\Theta}_{C, k}\right]$ is written as

$$
g_{k}\left[\mathbf{x}_{1},\left.\mathbf{x}_{2}\right|_{t}, \boldsymbol{\Theta}_{C, k}\right]=C_{k}\left[\mathbf{x}_{1},\left.\mathbf{x}_{2}\right|_{t}, \boldsymbol{\Theta}_{C, k}\right]-D_{k} \quad k=\delta, v
$$

where $D_{k}$ denotes the given demand for the $k$ th failure mode. The fragility is then written as

$$
F\left[\mathbf{x}_{1},\left.\mathbf{x}_{2}\right|_{t}, \boldsymbol{\Theta}_{C}\right]=P\left[\bigcup_{k}\left\{g_{k}\left[\mathbf{x}_{1},\left.\mathbf{x}_{2}\right|_{t}, \boldsymbol{\Theta}_{C, k}\right] \leq 0\right\} \mid D_{k}\right]
$$

The uncertainties in the fragility arise from the inherent randomness, and the measurement and model errors in the capacity variables, the inexact nature of the limit state model $g_{k}\left[\mathbf{x}_{1},\left.\mathbf{x}_{2}\right|_{t}, \boldsymbol{\Theta}_{C, k}\right]$ (or its sub-models), and the uncertainties inherent in the model parameters $\boldsymbol{\Theta}_{C, k}$. Following Gardoni et al. (2002), predictive fragilities that account for the above uncertainties are developed. To explicitly reflect the influence of 
the epistemic uncertainties in $\boldsymbol{\Theta}_{C, k}$, the approximate \pm 1 standard deviation (SD) confidence bounds on the fragility are also developed by first-order analysis. The bounds approximately correspond to $15 \%$ and $85 \%$ probability levels.

\subsection{Application}

As an application of the developed capacity models, the fragility estimates for the RC column in the Lavic Road Overcrossing bridge are assessed, accounting for its deterioration over time. This concrete box-girder bridge is selected because much effort has been made in estimating the deterioration of its flexural stiffness using NDT (Stubbs et al. 1999; Park et al. 2001; Choi et al. 2004; Bolton et al. 2005). The bridge was built in 1967 in San Bernardino County, California, 7 miles west of Ludlow town. It passes over Interstate I-40 and is North-South oriented. The bridge is supported by abutments on the north and south ends and at approximately mid-span by one circular column with a diameter of $1,524 \mathrm{~mm}$ that seats on a spread footing. Additional details of the bridge can be found in Stubbs et al. (1999). The bridge was tested four times (in Dec. 1997, Sep. 1998, Sep. 1999, and Oct. 1999), exciting the bridge using a drop weight impact hammer. The responses of the bridges were measured by accelerometers. Based on the recorded response data, the eigenmodes were extracted using MEScope software (Stubbs et al. 1999). Then, the damage index method developed by Stubbs et al. (1996) was used to estimate the local stiffness change of the column. The Hector Mine Earthquake of magnitude 7.1 occurred between the last two measurements causing moderate damage 
to the bridge. The proposed approach is used to assess the change in fragility due to the damage from the earthquake.

Table 2-1 summarizes the flexural stiffness of the column according to the measurements by Choi et al. (2004). Among these four measurements, the first one (Dec. 1997) is considered to be accurate and is close to the calculated value based on the push-over analysis performed using the design parameters. The next three measurements (Sep. 1998, Sep. 1999, and Oct. 1999) were taken with a different set of instruments (Bolton et al. 2001, 2005) and lead to lower flexural stiffness than the first ones. To remove the bias in the 1998 and 1999 measurements, the measurements taken before the Hector Mine Earthquake (Sep. 1998 and Sep. 1999) are scaled up to the Dec. 1997 value. The same scaling factor for the Sep. 1999 measurement is then used for the measurement after the earthquake (Oct. 1999). Table 2-1 also summarizes the flexural stiffness calculated using the scaling factor.

Table 2-1. Flexural stiffness of the example column, EI $\left(10^{6} \cdot \mathrm{kN} \cdot \mathrm{m}^{2}\right)$

\begin{tabular}{ccccc}
\hline & Dec. 1997 & Sep. 1998 & Sep. 1999 & Oct. 1999 \\
\hline Original $^{1}$ & 4.61 & 2.65 & 2.62 & 2.15 \\
Scaled & 4.61 & 4.61 & 4.61 & 3.77 \\
\hline
\end{tabular}

1. Choi et al. (2004)

The numerical calculations for the fragility estimation are conducted using OpenSees software. OpenSees is a comprehensive, open-source, object-oriented finite element software. Haukaas and Der Kiureghian (2004) extended this software with 
reliability and sensitivity analysis capabilities. The column in this study is modeled in OpenSees by fiber-discretized cross-sections where each fiber contains a uniaxial inelastic material model.

Table 2-2. Distribution, mean and coefficient of variation $(\mathrm{COV})$ for the random variables in the model

\begin{tabular}{cccc}
\hline $\begin{array}{c}\text { Random } \\
\text { Variables }\end{array}$ & Mean & COV & Distribution \\
\hline$f_{c}^{\prime}$ & $20.7(\mathrm{MPa})^{1}$ & $5 \%^{2}$ & Lognormal \\
$f_{y}$ & $276(\mathrm{MPa})$ & $5 \%$ & Lognormal \\
$f_{y h}$ & $276(\mathrm{MPa})$ & $5 \%$ & Lognormal \\
$P$ & $2,641.3(\mathrm{kN})^{3}$ & $25 \%$ & Normal \\
$H$ & $7,467.6(\mathrm{~mm})$ & $1 \%$ & Lognormal \\
$D_{g}$ & $1,524(\mathrm{~mm})$ & $2 \%$ & Lognormal \\
$S$ & $101.6(\mathrm{~mm})$ & $5 \%$ & Lognormal \\
cover & $38.1(\mathrm{~mm})$ & $10 \%$ & Lognormal \\
\hline
\end{tabular}

1. Corresponds to the pre-event value

2. To account for uncertainties from measurement and model errors

3. Corresponds to $7 \%$ of the axial capacity based on the gross cross-section area

The decrease in the equivalent flexural stiffness of the column after the Hector Mine Earthquake is assumed to be due only to the strength and stiffness reduction in concrete associated to cracking and it is assumed that the properties of the reinforcement steel are not affected by the earthquake. Concrete compressive strength $f_{c}^{\prime}$ is adjusted so that EI from the moment curvature of the cross-sections with elastic-perfectly plastic idealization matches EI $\left.\right|_{t}$ obtained from the NDT. Thus, based on the estimated 
flexural stiffness from the Sep. 1999 measurement and the Oct. 1999 measurement, one can find $\left.f_{c}^{\prime}\right|_{t 1}$ and $\left.f_{c}^{\prime}\right|_{t 2}$ for the pre- and post-earthquake conditions, respectively.

After assessing $f_{c}^{\prime}$, to account for the uncertainties in the material properties, geometry, and applied axial force, the quantities in Table 2-2 are considered as random variables. Table 2-2 also provides the assumed distribution for each random variable, their mean and coefficient of variation (COV).

Figures 2-4 and 2-5 show the predictive fragility estimates of the example column for varying deformation and shear demands based on the estimated flexural stiffness pre- (thin solid line) and post-earthquake (thick solid line). It is shown that the fragility increases for both deformation and shear failure modes because of the reduction in the deformation and shear capacity due to the damage induced by the Hector Mine Earthquake. Table 2-3 gives the mean shear and deformation capacities, pre- to postearthquake. The results show that the damage introduced by the earthquake has a larger effect on the shear capacity than the deformation capacity.

In order to explicitly show the effect of the epistemic uncertainty in the model parameters, $\boldsymbol{\Theta}_{k}, \pm 1$ SD bounds are provided. The dashed lines in Figure 2-4 and 2-5 represent the bounds that approximately correspond to $15 \%$ and $85 \%$ probability levels. The dispersion indicated by the slope of the solid curve represents the effect of the aleatory uncertainty present in the random variables in Table $2-2, \varepsilon_{\delta}$, and $\varepsilon_{v}$. The dispersion indicated by the confidence bounds represents the influence of the epistemic uncertainty present in the model parameters $\boldsymbol{\Theta}_{k}$. 


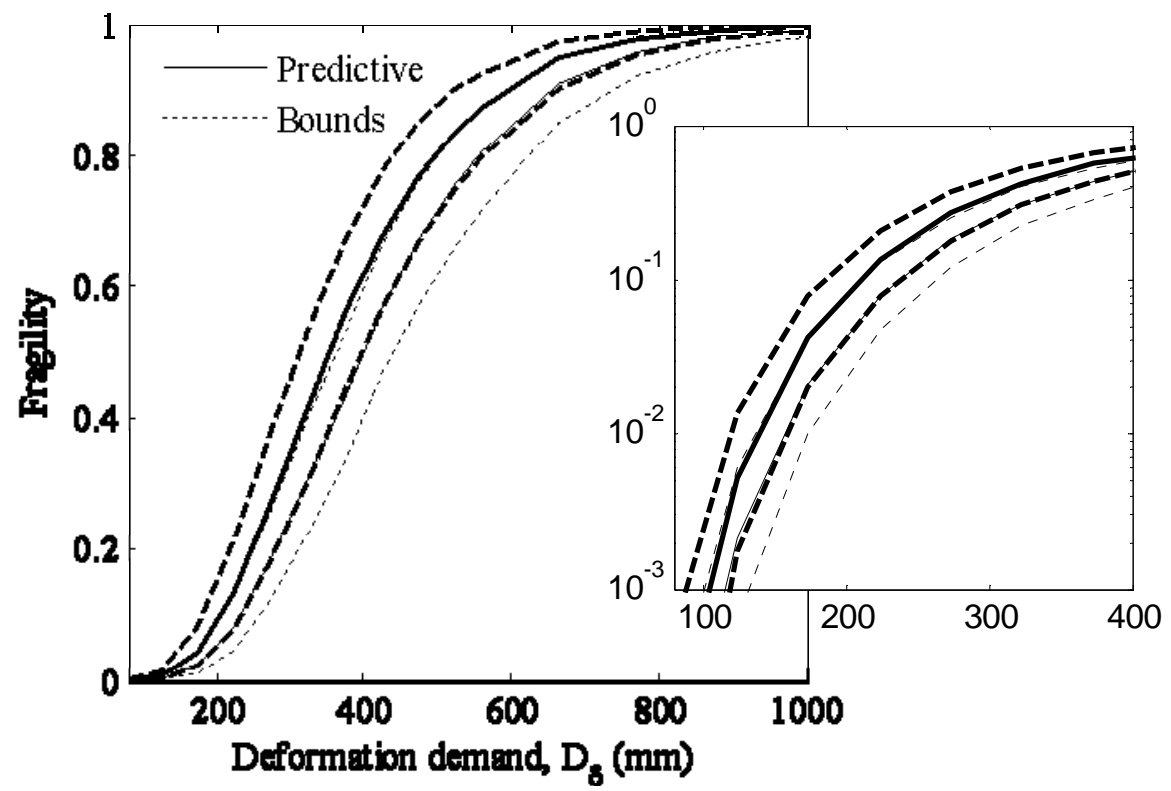

Figure 2-4. Fragility estimate with approximate confidence bounds for the deformation failure of the example column, pre- (thin lines) and postearthquake (bold lines)

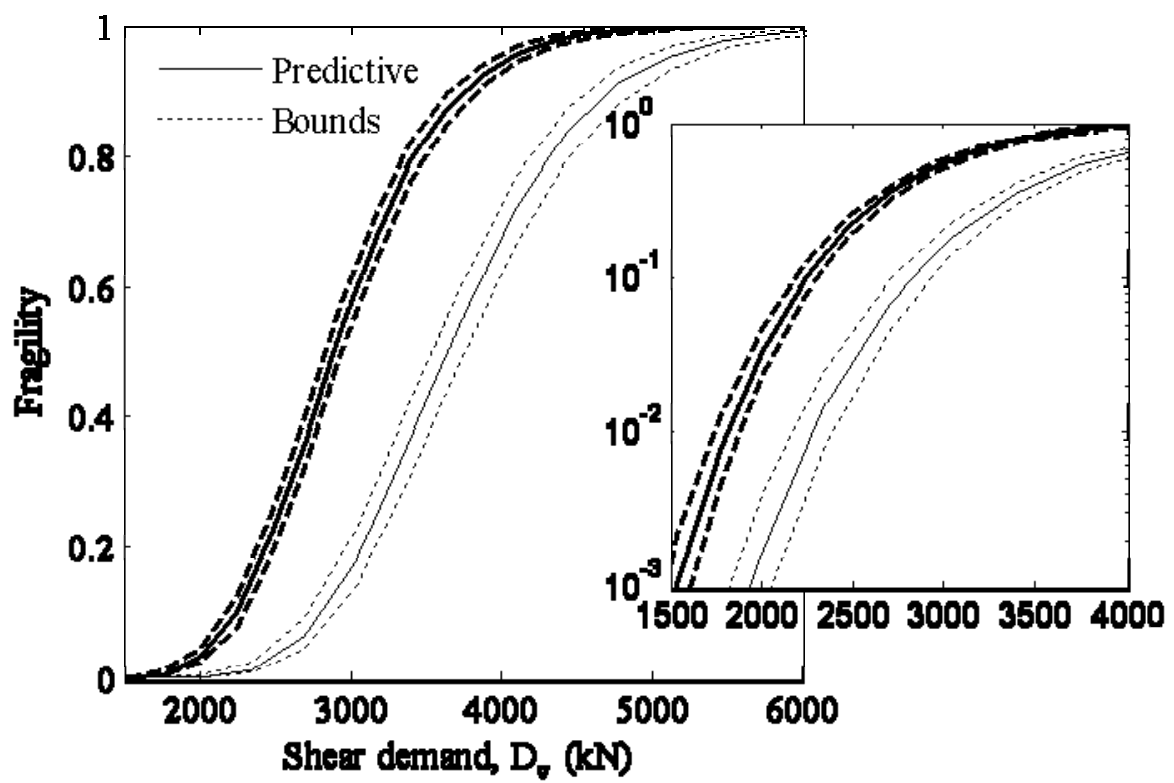

Figure 2-5. Fragility estimate with approximate confidence bounds for the shear failure of the example column, pre- (thin lines) and postearthquake (bold lines) 
Table 2-3. Mean deformation and shear capacity pre- and post-earthquake

\begin{tabular}{cccc}
\hline Capacity & $\begin{array}{c}\text { Pre- } \\
\text { earthquake }\end{array}$ & $\begin{array}{c}\text { Post- } \\
\text { earthquake }\end{array}$ & $\begin{array}{c}\text { Percentage } \\
\text { Change }\end{array}$ \\
\hline Deformation (mm) & 396.36 & 352.03 & $11.18 \%$ \\
Shear $(\mathrm{kN})$ & 3701.35 & 2892.31 & $21.86 \%$ \\
\hline
\end{tabular}

Figure 2-6 shows a comparison between the contour lines of the predictive bivariate fragilities, pre- and post-earthquake. Each contour line in this figure connects pairs of values of the demands $D_{\delta}$ and $D_{v}$ that are associated with a level of fragility in the range $0.1 \sim 0.9$. Consistently with what is observed for the univariate fragilities in Figures 2-4 and 2-5, Figure 2-6 shows that the damage induced by the Hector Mine Earthquake increases the probability of failure in the shear mode more significantly than in the deformation mode.

\subsection{Conclusions}

Probabilistic deformation and shear capacity models for RC bridge columns are developed to incorporate field information from NDT. The proposed models can be used when the flexural stiffness decays non-uniformly over the column height. The probabilistic models are used to assess the conditional probability of failure of reinforced concrete bridge columns for given deformation and shear demands.

The proposed formulation avoids using deterioration models and uses material properties estimated from the field data. This has two main advantages over the 
previous approaches 1) actual values for the material properties are used instead of assumed values; and 2) the approach accounts for all possible causes of deterioration instead of the mechanisms captured by the deterioration models. Furthermore, the approach takes into account the relevant sources of uncertainties including measurement and model errors. Although this study focuses on incorporating information on the flexural stiffness, the methodology presented in this section can be extended to account for other structural properties that might be obtained from NDT.

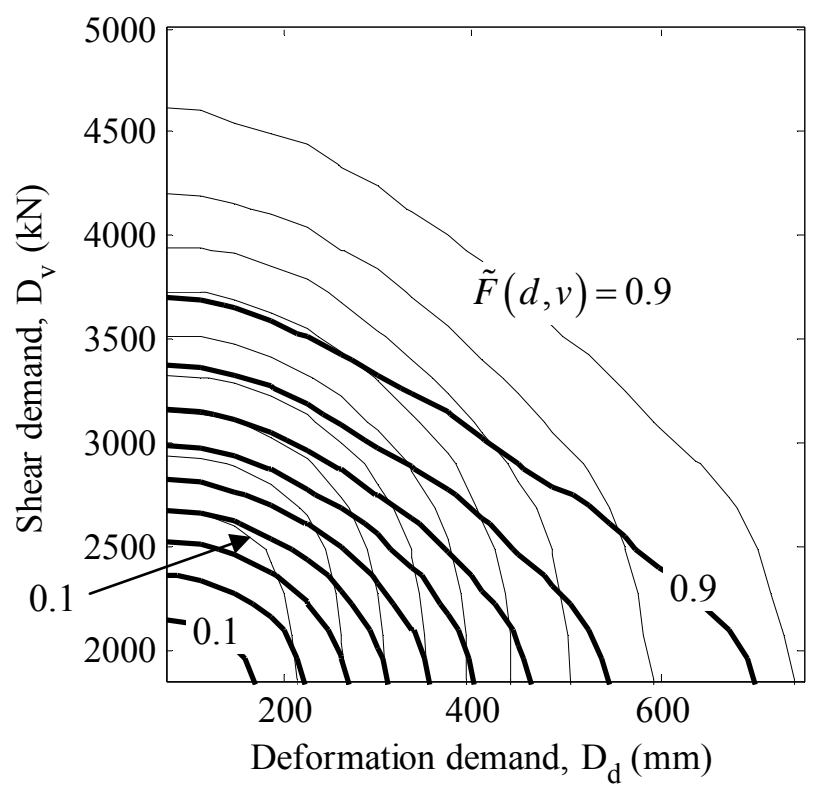

Figure 2-6. Contour plot of predictive deformation-shear fragility surface of the example column, pre- (thin lines) and postearthquake (bold lines) 
As an illustration, a case study is carried out to estimate the univariate deformation and shear fragilities and the bivariate deformation-shear fragility for the column in the Lavic Road Overcrossing bridge. This RC bridge was subject to the Hector Mine Earthquake in 1999. Pre- and post-earthquake fragility estimates are computed and compared. The results show that both the deformation and shear fragility increase due to the damage from the earthquake event. Furthermore, the analysis of the example column shows that the shear capacity degrades more rapidly than the deformation capacity. These results indicate that columns designed to fail in deformation, as per the current Caltrans' seismic design criteria, might fail in shear after being damaged by past earthquakes. However, a conclusive statement on this regard cannot be made based on the available results since the fragilities are computed for given deformation and shear demands. 


\section{PROBABILISTIC DEMAND MODEL}

\subsection{Introduction}

The need for incorporating performance-based engineering concepts into bridge design and rehabilitation has been widely recognized (e.g., Cornell and Krawinkler, 2000; Mackie and Stojadinović, 2003; Moehle and Deierlein, 2004). Seismic demand models are a critical component in performance-based seismic design and seismic risk assessment. Nonlinear static procedures (NSPs) are commonly used to predict the seismic demands by making use of force-deformation curves generated from nonlinear static pushover analysis. Commonly used NSPs include the capacity spectrum method (CSM) by the Applied Technology Council (ATC 1996), the coefficient method (CM) proposed by FEMA-273 (BSSC 1997), and the N2 method proposed by Fajfar (2000). FEMA-440 (ATC 2005) improves CSM and CM, and presents a preliminary evaluation of the improved methods. The N2 method can be considered as a special form of the CSM and has been adopted in the Eurocode-8 (CEN 2001). On the other hand, many researchers (e.g., Gardoni et al. 2003; Goel and Chopra 2004; Kunnath and Kalkan 2004; Kalkan and Kunnath 2006; Akkar and Metin 2007; Goel 2007; Zhong et al. 2008) have pointed out the drawbacks of these simplified static procedures. In particular, Heintz and Miranda (2007) attributed the inaccuracy of NSPs to (1) the unjustified use of the "equal displacement" rule in the short period range, (2) the use of a static load pattern, and (3) the negligence of the cyclic material strength and stiffness degradation, the dynamic $P-\Delta$ effect and instability, the multi-degree of freedom (MDOF) effects, and 
the effects from soil-structure interaction. Additionally, NSPs do not account for the uncertainties and variability in the ground motions and in the structural responses.

Probabilistic seismic demand models aim at capturing the uncertainties in the ground motions and the structure dynamic responses. Vamvatsikos and Cornell (2002) developed the incremental dynamic analysis (IDA) to estimate the structural demand given the pseudo-spectral acceleration, $P S A$, at the first mode period, $T_{1}$. However, IDA requires a series of time history analyses for any given structure to account for the variability in the ground motion and does not account for the uncertainties in the structural properties. Gardoni et al. (2002) proposed a general Bayesian methodology to construct probabilistic models that account for any source of information, including field measurements, laboratory data, and engineering judgment. Following this Bayesian methodology, Gardoni et al. (2003) and Zhong et al. (2008) constructed probabilistic seismic demand models for RC bridges that account for the prevailing uncertainties such as uncertainties in the structural properties, statistical uncertainties, and model errors. In particular, Gardoni et al. (2003) developed probabilistic demand models for general RC bridges with single-column bents and Zhong et al. (2008) developed demand models for RC bridges with two-column bents. However, Gardoni et al. (2003) and Zhong et al. (2008) used limited laboratory data on RC bents and one numerical model of a full bridge to calibrate the proposed demand models. Additionally, the dependence of the demand parameters on the ground motion characteristics is not complete, because the effects from the soil profile are not accounted for and near-field earthquakes are not considered. 
Krawinkler et al. (2003) studied the dependence of seismic deformation and ductility demand on earthquake magnitude and source-site distance. They found that one seismic intensity is adequate to describe ordinary ground motions but not for nearfield earthquakes, indicating that additional seismic intensities should be considered. Consistently, Luco (2002) used a combination of two intensity measures to account for the effect of the near-field ground motions on the nonlinear structural responses. More generally, Mackie and Stojadinović (2003) conducted a sensitivity analysis to explore the effect of different ground motion intensities on the seismic demands of RC bridges.

This study considers the randomness from ground motions and incorporates the prevailing uncertainties, such as the uncertainties in the structural properties, statistical uncertainties, and model errors, to develop probabilistic seismic demand models. The formulation of the proposed probabilistic demand models is constructed by adding correction terms to existing conventional deterministic demand models. The correction terms are used to correct the bias and improve the accuracy in the selected deterministic models. The model parameters are estimated through a Bayesian updating approach based on the virtual experiment demand data obtained from nonlinear analysis of detailed finite element models (FEMs) subjected to a set of representative ground motion records, including near-field records and considering the effects from soil characteristics. The FEMs simulate RC highway bridges with one single-column bent, typical of current construction in California. A computer experiment design method is used to construct 60 representative bridge configurations. Finally, an all possible subsets model selection is used to select the correction terms that contribute most to the demand prediction. 
Although the proposed model only focuses on typical RC highway bridges with one single-column bent in California, the same methodology is readily applied to other classes of bridges, when experiment demand data of another class of bridges become available.

Following the introduction, firstly how the virtual experiment demand data are obtained from numerical simulations is described. Next, the formulation of the proposed probabilistic demand models is introduced. Then, the model assessment is presented. Finally, the developed seismic demand models are illustrated by estimating the fragilities of an example bridge.

\subsection{Virtual Experiment Demand Data}

Due to the limited experimental or field data on full-scale bridges, this study uses computer-based simulations to evaluate the performance of RC bridges with one singlecolumn bent. The term "virtual experiment" herein refers to a nonlinear dynamic analysis performed on detailed bridge FEMs. In this section, 60 representative configurations of RC bridges with one single-column bent are generated using a Latin hypercube sample technique. Then how the ground motion records are selected is described. Finally, it shows how the FEMs are built and how the ground motions are assigned to the 60 bridge configurations.

\subsubsection{Latin Hypercube Sampling}

To reduce the high computational cost of nonlinear dynamic analyses, experimental 
design can be used to select representative bridge configurations. The experimental design in virtual experiments is different from classical experimental design since the computer code or program is deterministic; therefore, there is no random error and no replication is required. The design space is defined as the region bounded by the upper and lower limits of each design (input) variable being studied (Simpson et al. 2001). The goal of an experimental design for the virtual experiments is to spread the design variables as far from each other as possible while staying in the design space. This study adopts the Latin hypercube sampling technique introduced by McKay et al. (1979). For a fixed sample size, the Latin hypercube sampling technique maximizes the minimum distance between the design points and evenly spaces each design point in its range to give a good coverage of the design space.

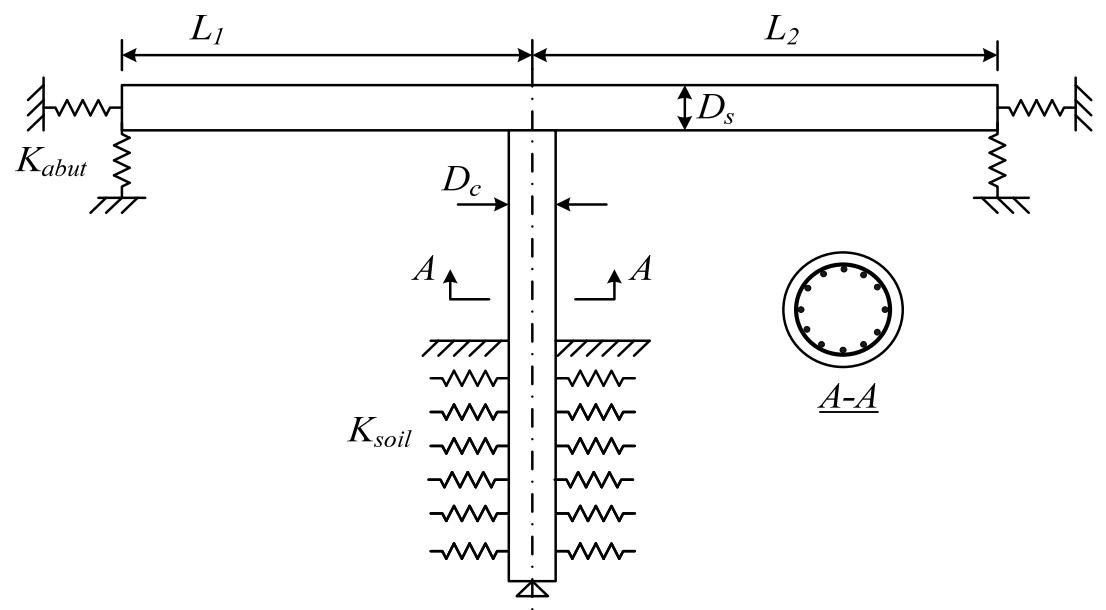

Figure 3-1. Typical one-bent column RC highway bridge configuration

12 parameters to characterize each bridge configuration are considered as shown in Figure 3-1. These parameters are the inputs for the experimental design. Table 3-1 
provides the considered ranges of the parameters, which are typical of current practice. The sample size is chosen based on the practical rule of about 5 samplings per design variable unless the output is quite variable over the range of that input variable. Therefore, 60 bridge configurations are generated by Latin hypercube sampling using the statistical software JMP version 7 (2007). The design parameters for the 60 bridges can be found in Appendix B. Note that each individual bridge configuration is not intended to correspond to any specific existing bridge.

Table 3-1. Ranges of the design parameters for typical highway bridges with one singlecolumn bent

\begin{tabular}{lc}
\hline \multicolumn{1}{c}{ Design Parameter } & Range \\
\hline Degree of skew, $\alpha$ & $0-60^{\circ}$ \\
Span (the shorter one), $L_{1}$ & $18-55 \mathrm{~m}$ \\
Column height, $H_{c}$ & $5-11 \mathrm{~m}$ \\
Column diameter-to-superstructure depth ratio, $D_{c} / D_{s}$ & $0.67-1.33$ \\
Reinforcement nominal yield strength, $f_{y}$ & $276-655 \mathrm{MPa}$ \\
Concrete nominal strength, $f_{c}^{\prime}$ & $20-55 \mathrm{MPa}$ \\
Longitudinal reinforcement ratio (column), $\rho_{l}$ & $1-4 \%$ \\
Transverse reinforcement ratio (column), $\rho_{s}$ & $0.4-1.1 \%$ \\
Additional bridge dead load, $w_{t}$ & $10-75 \%$ self-weight \\
Pile soil stiffness, $K_{\text {soil }}$ & (USGS) A, B, C, D \\
Abutment models, $K_{a b u t}$ & A, B, C, D, E, F, G \\
Two-span ratio, $L_{2} / L_{1}$ & $1.0-1.5$ \\
\hline
\end{tabular}

\subsubsection{Ground Motions}

To properly assess the seismic demand variables of interest and their associated uncertainties, it is critical to select representative ground motions for the virtual 
experiments. In particular, as pointed out by Krawinkler et al. (2003), the selected ground motion records should capture the characteristics of the possible seismic hazards, including their return periods, intensities, frequency contents, and durations. In this study, representative earthquake records are selected from the PEER (Pacific Earthquake Engineering Research Center) database. Each selected record has two orthogonal horizontal components and one vertical component. Thus, a fully three-dimensional dynamic analysis can be performed. Following Shome and Cornell (1999), the selected ground motions are subdivided into five bins based on moment magnitude, $M$ and the closest distance between the record location and the rupture zone, $R$. Each bin represents specific combinations of the earthquake characteristics and the collection of all bins captures all possible characteristics. Thus, each bin should have (1) enough earthquakes to capture the variability of the characteristics of that bin, and (2) the same number of ground motions as each of the other bins to provide an even representation of the possible characteristics without introducing bias into the ground motion characteristic or the assessment of the seismic demand variables of interest. Furthermore, ground motions within bins can be scaled up to higher intensities, without introducing bias.

The following five bins are considered:

1. Bin-I (SMSR: small $M$ and small $R$ ): $M=[5.5,6.5]$, and $R=[15 \mathrm{~km}, 30 \mathrm{~km})$

2. Bin-II (SMLR: small $M$ and large $R$ ): $M=[5.5,6.5]$, and $R=[30 \mathrm{~km}, 50 \mathrm{~km}]$ 
3. Bin-III (LMSR: large $M$ and small $R$ ): $M=(6.5,7.5]$, and $R=[15 \mathrm{~km}, 30 \mathrm{~km})$

4. Bin-IV (LMLR: large $M$ and large $R$ ): $M=(6.5,7.5]$, and $R=[30 \mathrm{~km}, 50 \mathrm{~km}]$

5. Bin-V (NF: near-field): $M=[6.0,7.5]$, and $R=[0 \mathrm{~km}, 15 \mathrm{~km})$

The records in the first four bins with $R \geq 15 \mathrm{~km}$ are ordinary ground motions and the ones in Bin-V with $R<15 \mathrm{~km}$ are near-field ground motions. Near-field ground motions are separated from the ordinary ground motions because they can have unique effects on the structural responses such as directivity and fling-step (Bray and Rodriguez-Marek 2004). Particularly, Baker and Cornell (2008) proposed vector-valued intensity measures for near-field ground motions. In this study, distance $R$ alone is used to define the near-field ground motions. Additionally, to capture the rupture directivity effects in the near field ground motions, earthquake moments $M \geq 6.0$ are selected for Bin-V (Luco, 2002). 
Table 3-2. Number (percentage) of earthquake records in PEER Strong Motion Database for given GM and USGS soil classification (Luco, 2002)

\begin{tabular}{|c|c|c|c|c|c|}
\hline \multirow[b]{2}{*}{$\begin{array}{c}\text { Ground } \\
\text { Motion Group }\end{array}$} & \multirow[b]{2}{*}{ GM Classification } & \multicolumn{4}{|c|}{ USGS Classification } \\
\hline & & $\begin{array}{c}\mathrm{A} \\
V_{s} \geq 750 \mathrm{~m} / \mathrm{s}\end{array}$ & $\begin{array}{c}\text { B } \\
360 \leq V_{s}<750 \mathrm{~m} / \mathrm{s}\end{array}$ & $\begin{array}{c}\mathrm{C} \\
360 \leq V_{s}<750 \mathrm{~m} / \mathrm{s}\end{array}$ & $\begin{array}{c}\mathrm{D} \\
V_{s} \geq 180 \mathrm{~m} / \mathrm{s}\end{array}$ \\
\hline Group 1 & $\begin{array}{c}\text { A: rock }\left(V_{s}>600 \mathrm{~m} / \mathrm{s}\right) \\
\text { or every thin soil }(<5 \mathrm{~m}) \text { over rock } \\
\text { B: shallow soil } \\
\text { soil } 5 \text { to } 20 \mathrm{~m} \text { thick over rock }\end{array}$ & $42(93.33 \%)$ & $(43.30 \%)$ & $(7.91 \%)$ & $(0 \%)$ \\
\hline Group 2 & $\begin{array}{c}\text { C: deep soil in narrow canyon } \\
\text { soil }>20 \mathrm{~m} \text { think, canyon }<2 \mathrm{~m} \text { wide } \\
\text { D: deep soil over broad canyon } \\
\text { soil }>20 \mathrm{~m} \text { think, canyon }>2 \mathrm{~m} \text { wide } \\
\text { E: soft soil }\end{array}$ & $(6.67 \%)$ & $(56.60 \%)$ & $(92.09 \%)$ & $(100 \%)$ \\
\hline Total & & $(100 \%)$ & $(100 \%)$ & $(100 \%)$ & $(100 \%)$ \\
\hline
\end{tabular}


To consider the effect of soil amplification due to different soil characteristics, the selected ground motions are divided into two groups as shown in Table 3-2 based on the soil types determined by Geomatrix (GM) site classification (PEER Strong Motion Database, 2000). Following the classification used by Abrahamson and Silva (1997), GM A and B are combined into the "rock and shallow" site category as ground motion Group 1 and GM C, D and E are combined into the "deep soil" site as Group 2. Each soil group has the five bins described above, which gives a total of 10 bins. For each bin, 20 ground motions are selected, for a total of 200 ground motions. Appendix C gives the selected ground motion records and record information.

Note that the shape of the pseudo acceleration spectrum of the first period, $P S A\left(T_{1}\right)$, can be used to assess the frequency content of the ground motions selected for each bin (Vamvatsikos and Cornell 2005). Therefore, the representativeness of the selected ground motions is checked by comparing the shape of the median of the computed $P S A\left(T_{1}\right)$ for each bin with the shape of the median $P S A\left(T_{1}\right)$ calculated based on Abrahamson and Silva (1997) attenuation law using the central values of the $M$ and $R$ ranges for each bin. Furthermore, the selected ground motions should be representative of the variability in the frequency content of each bin. Similar with the check on the median frequency content, the variability in the frequency content of the selected ground motions is checked by comparing the standard deviation of the computed $\operatorname{PSA}\left(T_{1}\right)$ for each bin with the standard deviation of the calculated $\operatorname{PSA}\left(T_{1}\right)$ using Abrahamson and Silva (1997) attenuation law using the central values of the $M$ and $R$ ranges for each bin. 

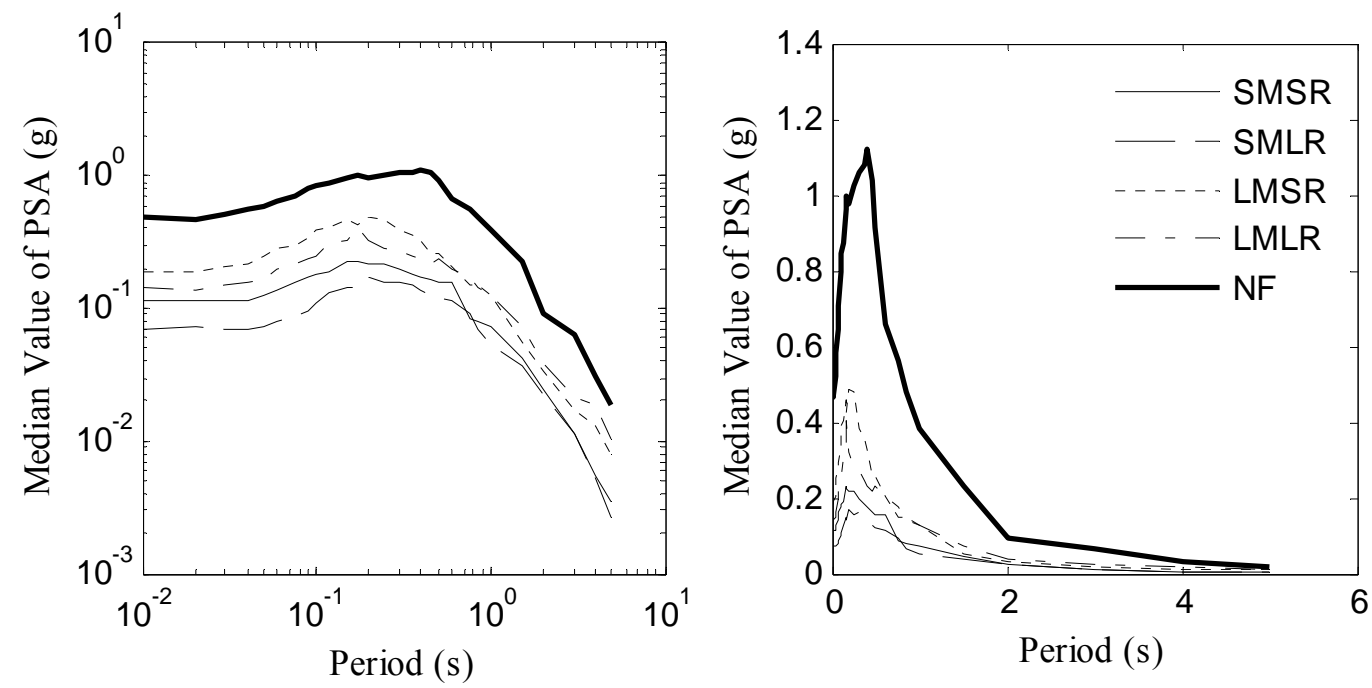

(a) Based on selected ground motions for each bin (left: log-log space; right: original space)
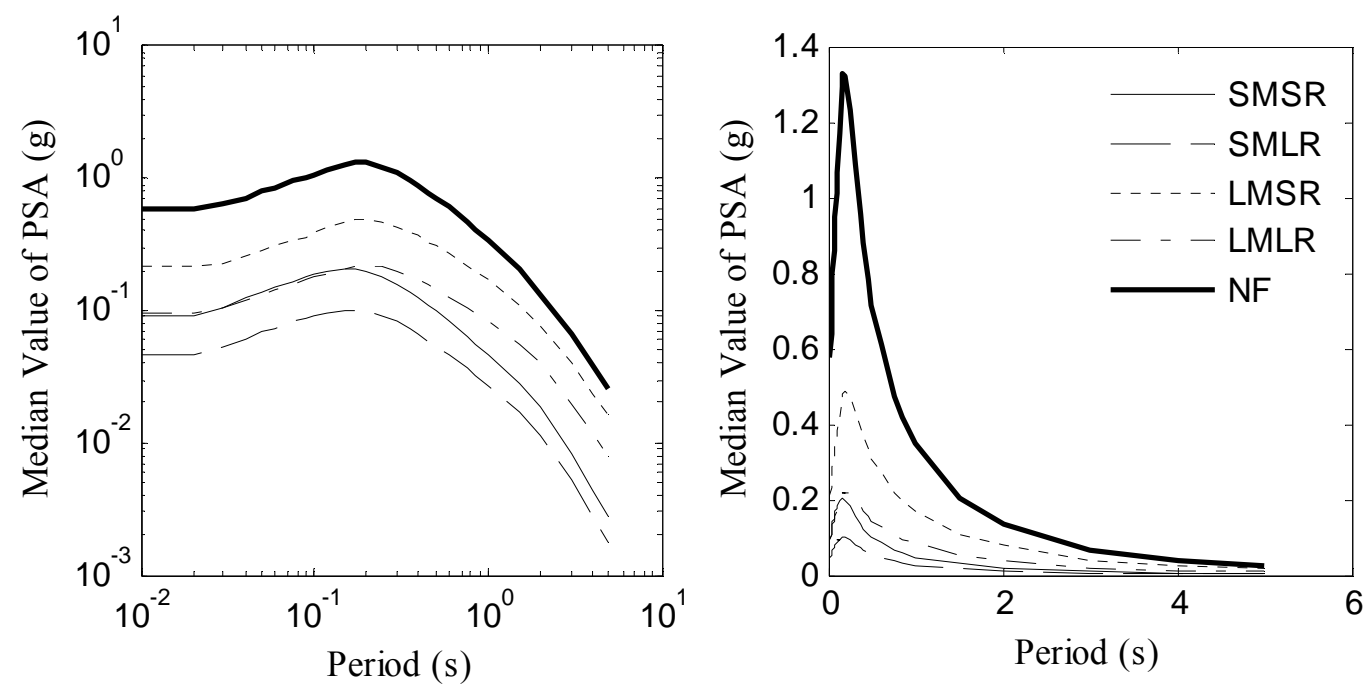

(b) Based on attenuation law by Abrahamson and Silva (1997) (left: log-log space; right: original space)

Figure 3-2. Median $\operatorname{PSA}\left(T_{1}\right)$ spectra for each bin with 5\% damping at rock and shallow soil site 

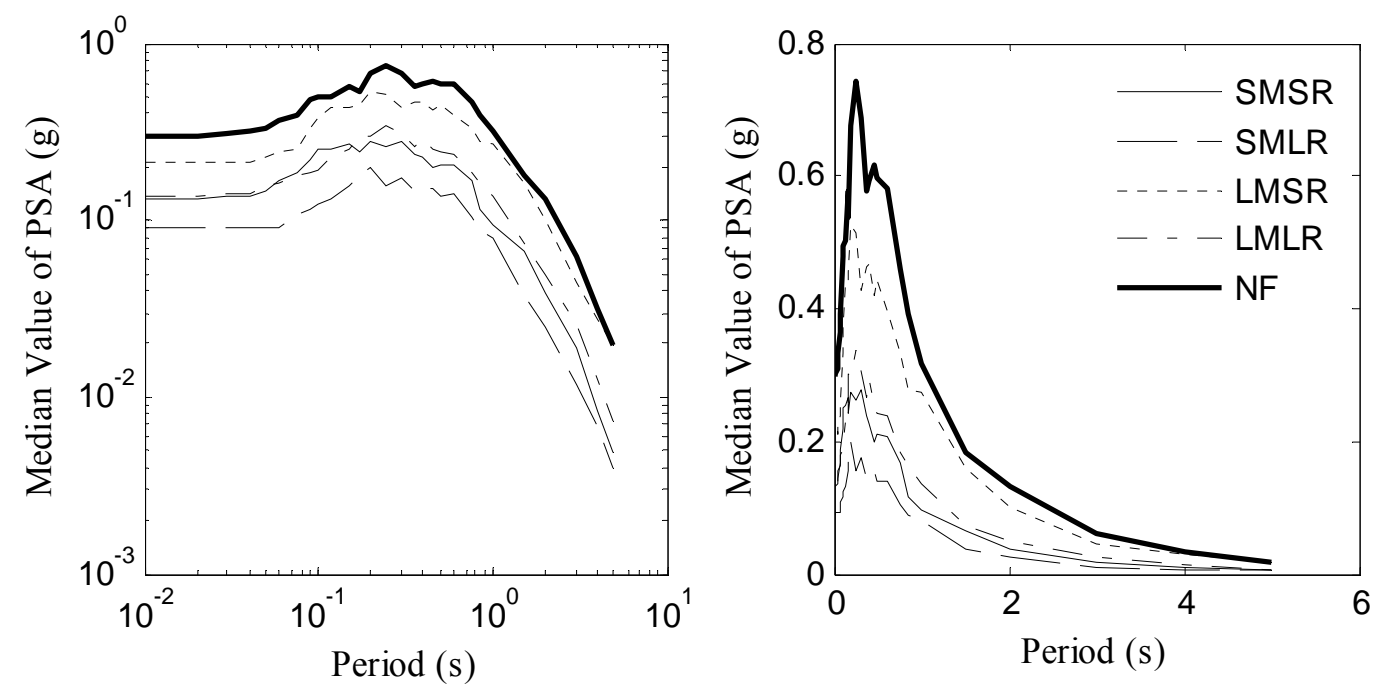

(a) Based on selected ground motions for each bin (left: log-log space; right: original space)
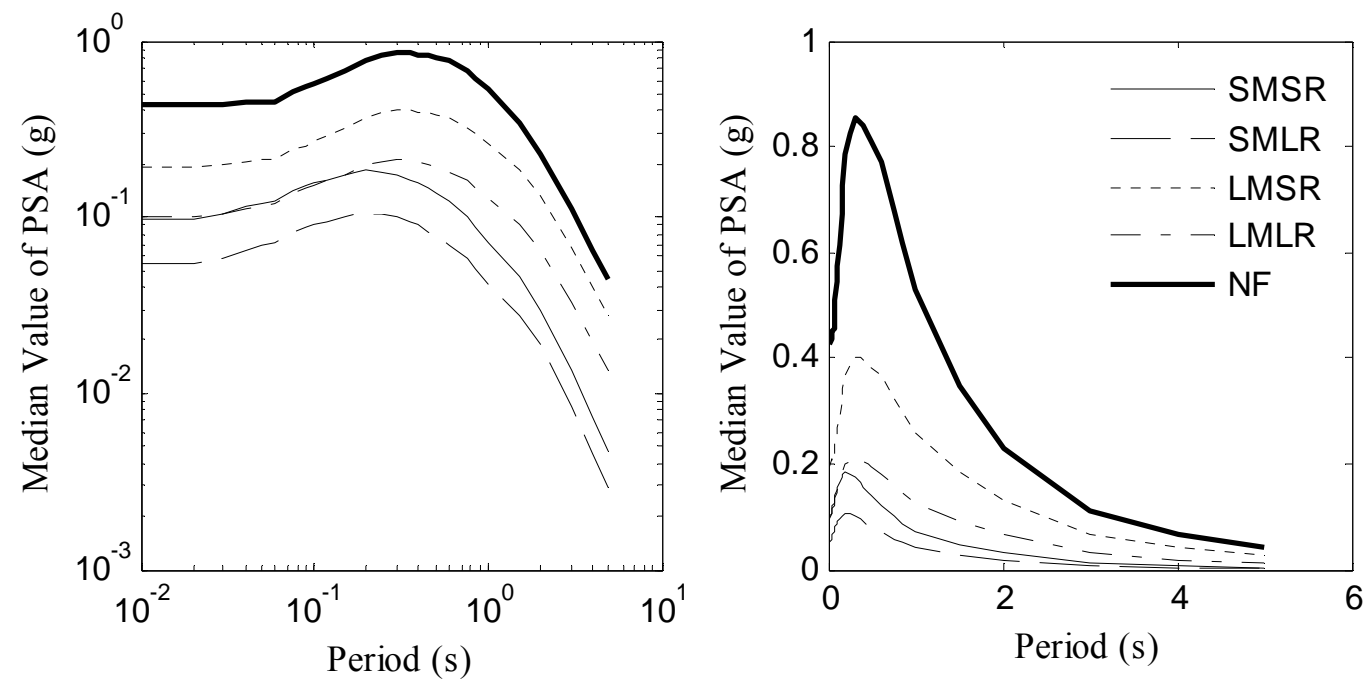

(b) Based on attenuation law by Abrahamson and Silva (1997) (left: log-log space; right: original space)

Figure 3-3. Median $P S A\left(T_{1}\right)$ spectra for each bin with 5\% damping at deep soil site

Figure 3-2 shows the median $\operatorname{PSA}\left(T_{1}\right)$ spectra based on the selected ground motions and Abrahamson and Silva (1997) attenuation law for Group 1. Similarly, Figure 3-3 shows the same quantities as in Figure 3-2 for Group 2. Figures 3-4 and 3-5 
show the comparisons of the standards deviation of $\operatorname{PSA}\left(T_{1}\right)$. The solid dots $(\bullet)$ represent the values calculated using the selected ground motions and the solid line represents the values calculated using Abrahamson and Silva (1997) attenuation law. The similarity in both the shapes of the median $P S A\left(T_{1}\right)$ spectra and the values of the standard deviations suggest that the selected ground motions are adequately representative of each bin.

In order to study the nonlinear responses of bridges due to larger earthquakes, the ground motions in each bin are scaled by multiplying the earthquake acceleration records by a constant. Followed the suggestion by Luco (2002), the ordinary ground motions (Bins I-IV) are scaled up by a factor of eight, and the near-field ground motions (Bin V) are scaled up by a factor of two such that "1-sigma level" of elastic displacements are approximately the same for the ordinary and near-field ground motions. Thus, in each bin, there are 20 un-scaled ground motions and 20 correspondingly scaled ground motions. 

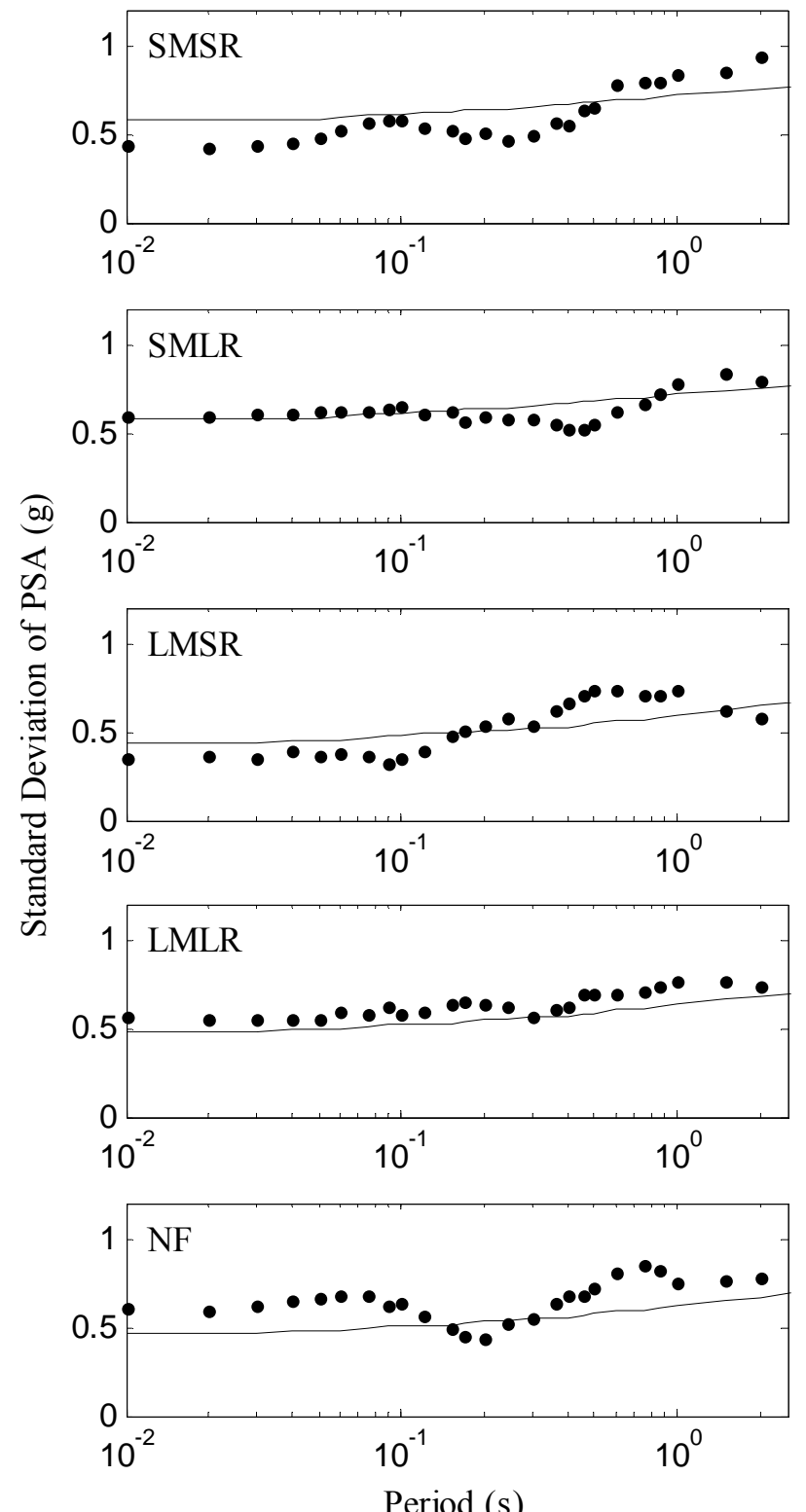

Figure 3-4. Comparison of standard deviation of $P S A\left(T_{1}\right)$ values obtained by 20 ground motions (dots) and standard deviation values based on attenuation law by Abrahamson and Silva (1997) (solid lines) for each bin with 5\% damping at rock and shallow soil site 

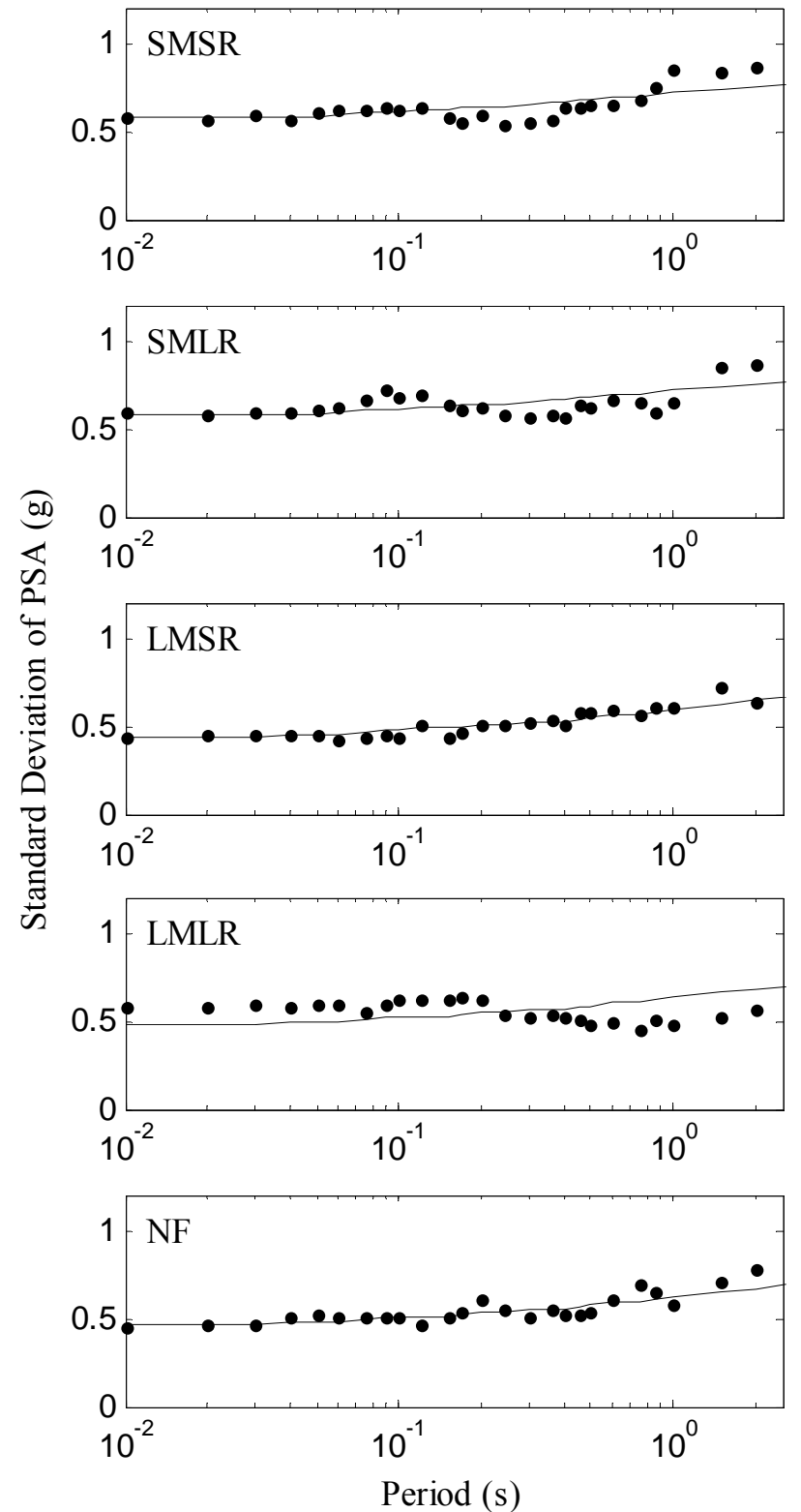

Figure 3-5. Comparison of standard deviation of $P S A\left(T_{1}\right)$ values obtained by 20 ground motions (dots) and standard deviation values based on attenuation law by Abrahamson and Silva (1997) (solid lines) for each bin with 5\% damping at deep soil site 


\subsubsection{Finite Element Models}

The OpenSees platform (McKenna and Fenves 2000) is used in this study for constructing the FEMs of the bridges and conducting the nonlinear time history analysis. Sixty FEMs are generated for the bridge configurations described above. In the FEMs, the bridge piles are modeled using bilinear elastic-perfectly plastic springs. To reflect the variability in the soil type, four different stiffnesses are considered based on the USGS (U.S. Geological survey) soil classification. The properties for each spring type can be found in Mackie and Stojadinović (2003). Instead of the GM soil classification that are used to divide the ground motions, the USGS soil classification is used for pile springs, because the USGS soil classification is based only on the top up to $30 \mathrm{~m}$ of soils.

Seat-type abutment is assumed in the longitudinal direction. The interaction between the abutment and the soil is modeled using gap-spring elements and bearing pad springs with elastic-perfectly plastic behavior. Seven abutment models of the gap-spring elements with different stiffness and strength properties are used. They are a simple roller support model (abutment type A), one model that follows Caltrans' specifications (2000) (abutment type B), two models (with and without participating mass) developed by Maroney et al. (1994) (abutment type C and type F), one model developed by Wilson and Tan (1990) (abutment type D), and two models (with and without participating mass) developed by Zhang and Makris (2001) (abutment type E and type G). An initial gap of $152.4 \mathrm{~mm}$ in the longitudinal direction and no gap in the transverse direction are assumed. The details on the stiffness and the participating mass for these abutment models can be found in Mackie and Stojadinović (2002). 
The bridge deck is designed as a 4-cell box girder $10.97 \mathrm{~m}$ wide, which is typical of bridges with single-column bents. The ratio between the depth of the box girder, $D_{s}$, and the longest span, $L_{2}$, is assumed to be 0.055 for all bridge configurations. This value is within the recommended range (0.05-0.06) in AASHTO (1992) for best appearance and economy. The box-girder superstructure is modeled by elastic beam elements, which is consistent with the assumptions in the current design practice in California. The column and pile shafts are modeled using nonlinear beam-column elements with fiber cross sections including $P-\Delta$ effects.

Table 3-2 shows the number and percentage of earthquake records in the PEER Strong Motion Database for given GM and USGS soil classification. Based on the correlation between GM and USGS shown in Table 3-2, in this study, bridges with USGS soil type A (USGS-A) are excited only by the records in Group 1, bridges with USGS-B are excited by the ground motions in both groups, and bridges with USGS-C and USGS-D are excited only by the ground motions in Group 2.

Instead of exciting 60 bridges with all earthquake records (a total of 200 ground motions for each group), 20 ground motions are randomly assigned to every bridge, two from each of the 10 bins. For the bridges with USGS-B, one ground motion from each of the 20 bins is selected. To ensure that all the ground motion records are used, the earthquake records are assigned to the bridges using a sampling without replacement. Because the orientation of the bridges with respect to the fault is unknown, one of the horizontal components of each ground motion record is randomly assigned to the bridge transverse direction and the other horizontal component to the bridge longitudinal 
direction.

\subsection{Formulation of Probabilistic Demand Models}

Following Gardoni et al. (2003), the probabilistic deformation and shear demand models are developed by adding correction terms to a selected existing deterministic model commonly used in practice. The models can be written as

$$
D_{k}\left(\mathbf{x}, \boldsymbol{\Theta}_{D, k}\right)=\hat{d}_{k}(\mathbf{x})+\gamma_{D, k}\left(\mathbf{x}, \boldsymbol{\theta}_{D, k}\right)+\sigma_{D, k} \varepsilon_{D, k} \quad k=\delta, v
$$

where $D_{k}\left(\mathbf{x}, \Theta_{k}\right)=$ demand measure (or a suitable transformation), $\hat{d}_{k}(\mathbf{x})=$ demand (or a suitable transformation) predicted by a selected deterministic demand model, $\gamma_{D, k}\left(\mathbf{x}, \boldsymbol{\theta}_{D, k}\right)=$ correction term for correcting the bias and random errors in $\hat{d}_{k}(\mathbf{x})$, $\boldsymbol{\Theta}_{D, k}=\left(\boldsymbol{\theta}_{D, k}, \sigma_{D, k}\right)=\mathrm{a}$ vector of unknown model parameters, $\boldsymbol{\theta}_{D, k}=\left(\theta_{D, k 1}, \ldots, \theta_{D, k} p_{k}\right)$, $\sigma_{D, k}=$ standard deviation of the model error, $\varepsilon_{k}=$ normal random variable with zero mean and unit variance, $\mathbf{x}=$ a vector of basic variables, e.g., material properties, member dimensions, and imposed boundary conditions. The index $k$ denotes the failure mode of interest, e.g., for deformation $(k=\delta)$ and for shear force $(k=v)$. Two assumptions are used in assessing the demand model in Eq. (3.1): the homoskedasticity assumption $\left(\sigma_{D, k}\right.$ is constant and independent of $\mathbf{x}$ ), and the normality assumption ( $\varepsilon_{D, k}$ has the Normal distribution). Both assumptions usually can be satisfied by using a variance stabilizing transformation of the demand quantities of interest (Box and Cox, 1964). Diagnostic plots can be used to check the suitability of the transformation (Rao and Toutenburg, 1997; Sheather, 2008). This study uses the natural logarithmic transformation of the 
normalized deformation and shear demands, that is $D_{\delta}=\ln \left(\Delta / H_{c}\right)$, where $\Delta=$ deformation demand, $H_{c}=$ height of the bridge column; $D_{v}=\ln \left[V /\left(f_{t}^{\prime} A_{c}\right)\right]$, where $V=$ shear demand,$f_{t}^{\prime}=$ tensile strength of concrete and $A_{c}=$ bridge column gross crosssectional area; $\hat{d}_{\delta}=\ln \left(\hat{\Delta} / H_{c}\right)$ and $\hat{d}_{v}=\ln \left[\hat{V} /\left(f_{t}^{\prime} A_{c}\right)\right]$ where $\hat{\Delta}$ and $\hat{V}$ are the deterministic estimates of the deformation and shear demands. In the following, it shows which existing deterministic demand models are selected and how to construct the candidate correction terms.

In this study, three existing deterministic procedures are considered to assess the demand quantities of interest: the improved CM described in FEM-440 (ATC 55 2005), the N2 method developed by Fajfar (2000), and the modified N2 (MN2) method developed by Gardoni et al. (2003). In these three methods, a MDOF system is transformed into an equivalent single-degree-of-freedom (SDOF) system through a nonlinear static pushover analysis using an assumed displacement shape and lateral load distributions. A nonlinear force-deformation curve for the equivalent SDOF is then generated. The N2 method generates the force-deformation curve taking the sum of all the shear reactions including those at the abutments. The MN2 method, on the other hand, only uses the sum of the shear forces in the columns. Not including the shear forces at the abutments was found to provide more accurate estimates of the shear demand in the bridge columns (Gardoni et al. 2003). In this study,equations developed by Krawinkler and Nassar (1992) for determining the inelastic displacement demand are used in the N2 and MN2 methods. CM is implemented using the pushover analysis from 
the MN2 methods and computing the transition from the elastic displacement to the inelastic displacement demand with modification factors (ATC 55 2005). With these three selected deterministic models, three corresponding probabilistic demand models are developed. Then, a comparison of the accuracy and complexity of the three probabilistic models is made to determine which demand model is the most effective.

The candidate correction terms are selected based on their relations to the residuals between the demand data and $\hat{d}_{k}(\mathbf{x})$. By correcting the bias in $\hat{d}_{k}(\mathbf{x})$, the developed demand models consider the nonlinear behavior and the inherent uncertainties of bridges, while retaining the simplicity of $\hat{d}_{k}(\mathbf{x})$ to facilitate their practical implementation. Therefore, the selected correction terms provide a way to complement $\hat{d}_{k}(\mathbf{x})$ with ground motion intensity measures, which are critical to determine the seismic demands, and give us an insight into the seismic behavior of structures. The correction term, $\gamma_{D, k}\left(\mathbf{x}, \boldsymbol{\theta}_{D, k}\right)$ in Eq. (3.1), can be expressed by a set of "explanatory" functions as follows:

$$
\gamma_{D, k}\left(\mathbf{x}, \boldsymbol{\theta}_{D, k}\right)=\sum_{j=0}^{p_{D, k}} \theta_{D, k} h_{k j}(\mathbf{x}) \quad k=\delta, v
$$

where $\boldsymbol{\theta}_{D, k}$ is a vector of unknown model parameters, $p_{D, k}+1=$ number of model parameters, and $h_{k j}(\mathbf{x})$ are the candidate normalized explanatory functions. Particularly $h_{k 0}(\mathbf{x})=1$ is selected to detect a potential constant bias, and $h_{k 1}(\mathbf{x})=\hat{d}_{k}(\mathbf{x})$ is used to capture the potential under- or over-estimate of the deterministic model. To account for different structural behavior before and after yielding, two correction terms are selected: 


$$
\begin{aligned}
& h_{k 2}(\mathbf{x})=\left[\hat{d}_{k}(\mathbf{x})-\hat{d}_{k, y}(\mathbf{x})\right]_{-}=\left\{\begin{array}{ll}
\hat{d}_{k}(\mathbf{x})-\hat{d}_{k, y}(\mathbf{x}) & \text { if } \hat{d}_{k}(\mathbf{x}) \leq \hat{d}_{k, y}(\mathbf{x}) \\
0 & \text { if } \hat{d}_{k}(\mathbf{x})>\hat{d}_{k, y}(\mathbf{x})
\end{array} \quad k=\delta, v\right. \\
& h_{k 3}(\mathbf{x})=\left[\hat{d}_{k}(\mathbf{x})-\hat{d}_{k, y}(\mathbf{x})\right]_{+}=\left\{\begin{array}{ll}
0 & \text { if } \hat{d}_{k}(\mathbf{x}) \leq \hat{d}_{k, y}(\mathbf{x}) \\
\hat{d}_{k}(\mathbf{x})-\hat{d}_{k, y}(\mathbf{x}) & \text { if } \hat{d}_{k}(\mathbf{x})>\hat{d}_{k, y}(\mathbf{x})
\end{array} \quad k=\delta, v\right.
\end{aligned}
$$

where $\hat{d}_{k, y}(\mathbf{x})=$ demand value at yielding predicted from the selected deterministic demand model.

Furthermore, since ground motion characteristics, such as the intensity, frequency content, and duration, could also play a critical role in the response of complex soil-structure systems (Krawinkler et al. 2003), the explanatory functions containing earthquake parameters should be included. Explanatory functions $h_{k 4}(\mathbf{x})$ through $h_{k 16}(\mathbf{x})$ are defined as the natural logarithm of the 12 intensity measures defined in Table 3-3.

In the Table 3-3, $T_{1}=$ natural frequency of equivalent SDOF for a bridge, $u_{g}(t)$, $\dot{u}_{g}(t)$, and $\ddot{u}_{g}(t)$ denote the ground motion displacement, velocity and acceleration at time $t$, respectively, $D=$ duration of a ground motion record, and $T_{D}=t\left(0.95 I_{A}\right)-t\left(0.05 I_{A}\right)=$ strong ground motion duration based on the time between 2.5 and 97.5 per cent of the Arias intensity, $I_{A}$ (Trifunac and Brady, 1975). An indicator variable, $D_{\text {soil }}$, is defined as $h_{k 17}(\mathbf{x})$ is used to classify the soil based on GM 
and can be expressed as,

$$
h_{k 17}(\mathbf{x})=D_{\text {soil }}=\left\{\begin{array}{ll}
0 & \text { for GM soil classification A or B } \\
1 & \text { for GM soil classification Cor D }
\end{array} \quad k=\delta, v\right.
$$

Finally, to account the potential dependence of the shear demand on the deformation

demand and vice versa, $h_{\delta 18}(\mathbf{x})=\hat{d}_{v}(\mathbf{x})$ and $h_{v 18}(\mathbf{x})=\hat{d}_{\delta}(\mathbf{x})$ are used. Note that all the candidate explanatory functions are dimensionless so that they can be used for any unit systems. Among the candidate explanatory functions, only those statistically important will be selected using a model selection process, which is described later.

\subsection{Model Assessment}

The demand values from the virtual experiments are randomly split into two groups. Two-thirds of the data (training data) are used to develop the demand models, and the remaining one-third of the data is used for model validation (validation data) to evaluate the performance of the developed models. A Bayesian updating rule (Box and Tiao 1992) is adopted to estimate the posterior statistics of the model parameters $\boldsymbol{\Theta}_{D, k}$ in Eq.

(3.1). Note that the problem of numerical instability in the virtual experiments can be handled by setting a threshold value for the demand of interest. Such threshold represents the maximum demand that can be obtained with sufficient confidence on the accuracy of the numerical analysis. The threshold value can then be taken as a lower bound demand datum in case the numerical instability occurs and the analysis exceeds the specified threshold. Details on how to incorporate lower bound data in the likelihood function in the Bayesian updating can be found in Gardoni et al. (2002) and 
Ramamoorthy et al. (2006). Furthermore, a model selection process is used to eliminate unimportant explanatory functions and select the ones that are needed for an accurate, unbiased, and parsimonious model.

Table 3-3. Candidate explanatory functions of normalized intensity measures

\begin{tabular}{ll}
\hline \multicolumn{1}{c}{ Intensity Measure Description } & \multicolumn{1}{c}{ Normalized Formula } \\
\hline Elastic spectral acceleration $^{\dagger}$ & $S_{a} / g$ \\
Elastic spectral velocity $^{\dagger}$ & $S_{v} T_{1} / H_{c}$ \\
Elastic spectral displacement & $S_{d} / H_{c}$ \\
Peak ground acceleration & $P G A / g=\max \left|\ddot{u}_{g}(t)\right| / g$ \\
Peak ground velocity & $P G V \cdot T_{1} / H_{c}=\max \left|\dot{u}_{g}(t)\right| \cdot T_{1} / H_{c}$ \\
Peak ground displacement & $P G D / H_{c}=\max \left|u_{g}(t)\right| / H_{c}$ \\
Cumulative absolute velocity & $C A V \cdot T_{1} / H_{c}=\left[\int_{0}^{D}\left|\ddot{u}_{g}(t)\right| \mathrm{d} t\right] \cdot T_{1} / H_{c}$ \\
Cumulative absolute displacement & $C A D / H_{c}=\left[\int_{0}^{D}\left|\dot{u}_{g}(t)\right| \mathrm{d} t\right] / H_{c}$ \\
Arias Intensity & $I_{A} \cdot T_{1} / H_{c}=(\pi / 2 g) \cdot \int_{0}^{D}\left[\ddot{u}_{g}(t)\right]^{2} \mathrm{~d} t \cdot T_{1} / H_{c}$ \\
Velocity Intensity & $C A D / H_{c}=1 / \dot{u}_{g, \max } \cdot \int_{0}^{D}\left[\dot{u}_{g}(t)\right]^{2} \mathrm{~d} t / H_{c}$ \\
Root mean square acceleration & $A_{r m s} / g=1 / T_{D} \cdot \int_{0}^{T_{D}}\left[\ddot{u}_{g}(t)\right]^{2} \mathrm{~d} t / g$ \\
Characteristic intensity & $I_{c} \cdot T_{1} / H_{c}=A_{r m s}^{1.5} T_{D} \cdot T_{1} / H_{c}$ \\
\hline
\end{tabular}

$\dagger$ at the first mode period in the transverse direction and with 5\% damping

Before conducting the model selection, the validity of the full models, which includes all the candidate explanatory functions, is checked using diagnostic plots. The diagnostic plots include plots of the residuals and of the roots of the standardized 
residuals vs. the fitted values, normal Q-Q plots of the standardized residuals, and plots of standardized residuals vs. the corresponding leverages (Sheather 2008). For each demand model, the first two diagnostic plots are used to check the homoskedasticity assumption, the normal Q-Q plot is used to check the normality assumption, and the last diagnostic plot can help identify outliners and bad leverage points. Additionally, marginal model plots (Cook and Weisberg 1997) are used to check that each explanatory function is modeled correctly in the corresponding model. Added variable plots (Mosteller and Tukey 1977) are used to assess the effect of each explanatory function on the predicted demands. However, since non-constant standardized residuals are still observed in the diagnostic plots for the shear models after using natural logarithmic transformation suggested by Box and Cox (1964), a weighted least squares regression (Sheather 2008) is used to handle the remaining heteroskedasticity (non-constant variance) of the models.

For model selection, with a number of $p$ predictors, the traditional stepwise methods (either backward or forward) consider at most $p(p+1) / 2$ possible predictor subsets, while all possible subsets model selection (Sheather 2008) determine the best model from all $2^{p}$ of the possible subsets. When there is high multicolinearity among the potential explanatory functions (such as the case in this study), instead of a traditional stepwise model section process the all possible subsets model selection process should be used. The all possible subsets model selection uses the adjusted $R^{2}$ $\left(R_{\text {adj }}^{2}\right)$, the Akaike's information criterion ( AIC ) (Akaike 1974), the corrected Akaike's 
information criterion $\left(A I C_{C}\right)$ (Hurvich and Tsai 1989), and the Bayesian information criterion (BIC) (Schwarz, 1978). The quantity $R_{a d j}^{2}$ is defined as

$$
R_{a d j}^{2}=1-\frac{\sum_{i=1}^{N_{s}}\left[D_{k, i}\left(\hat{\mathbf{x}}, \hat{\boldsymbol{\Theta}}_{D, k}\right)-d_{k, i}\right]^{2} /\left(N_{s}-N_{p}-1\right)}{\sum_{i=1}^{N_{s}}\left[D_{k, i}\left(\hat{\mathbf{x}}, \hat{\boldsymbol{\Theta}}_{D, k}\right)-\bar{d}_{k}\right]^{2} /\left(N_{s}-1\right)} \quad k=\delta, v
$$

where $D_{k, i}\left(\hat{\mathbf{x}}, \hat{\boldsymbol{\Theta}}_{D, k}\right)=$ a point estimate of the demand of interest predicted by the developed probabilistic model using the mean vectors of $\mathbf{x}$ and $\boldsymbol{\Theta}_{D, k}$, indicated as $\hat{\mathbf{x}}$ and $\hat{\boldsymbol{\Theta}}_{D, k}$, respectively, $d_{k, i}=$ the demand values from the virtual experiments, $\bar{d}_{k}=\sum_{i=1}^{N_{s}} d_{k, i} / N_{s}=$ the sample mean of $d_{k, i}$, and $N_{s}=$ sample size. The other three criteria $A I C, A I C_{C}$, and $B I C$ measure how well the model fits the data by trading off the complexity of the model with its accuracy. They can be written as

$$
\begin{gathered}
A I C=-2 \ln \left[L\left(\boldsymbol{\Theta}_{D, k}\right)\right]+2 N_{p} \quad k=\delta, v \\
A I C_{C}=A I C+\frac{2\left(N_{p}+2\right)\left(N_{p}+3\right)}{N_{s}-N_{p}-2} \\
B I C=-2 \ln \left[L\left(\boldsymbol{\Theta}_{D, k}\right)\right]+N_{p} \ln \left(N_{s}\right) \quad k=\delta, v
\end{gathered}
$$

where $L\left(\boldsymbol{\Theta}_{k}\right)=$ the maximum of the likelihood function for the estimated model.

For each subset of size $N_{p}$ (the number of explanatory functions used in the model) that ranges from 1 to $p_{k}+1$, the model selection identifies the model that maximizes $R_{a d j}^{2}$ and minimizes the other three criteria. The model with the highest $R_{a d j}^{2}$ 
and the lowest $A I C, A I C_{C}$, and $B I C$ is the most desirable among the competing models in the same subset. Notice that for each subset, the model that maximizes $R_{a d j}^{2}$ also minimizes $A I C, A I C_{C}$, and $B I C$. Thus there are $p_{k}+1$ candidate models to chose from, one for each subset. However, maximizing $R_{a d j}^{2}$ and minimizing $A I C, A I C_{C}$, and $B I C$ across all the subsets to select the overall most desirable model can give different answers because of the different penalties for the model complexity. Therefore, the candidate models are refitted using the validation data and the overall most desirable model is selected by checking the statistical significance (p-value) of the coefficients of the explanatory functions. All the models that have at least one $p$-value $\geq 0.05$ are removed from the pool of candidate models because this is an indication that the model is over fitting the data. Additionally, the F-test (Sheather 2008) is used to select the most parsimonious model among the remaining ones without under-fitting the data.

To verify the validity of the selected model, the estimates of $\boldsymbol{\Theta}_{k}$ based on the training data are compared with the estimates based on the validation data. The consistency in the estimates indicates that the selected model can be applied to data beyond those used to construct and assess the model. The Mean Absolute Percentage Error $(M A P E)$ can be used as an intuitive measure of the accuracy of the model and is defined as

$$
M A P E=\frac{1}{100 N_{s}}\left[\sum_{i=1}^{N_{s}}\left(\frac{\left|D_{k, i}\left(\hat{\mathbf{x}}, \hat{\boldsymbol{\Theta}}_{D, k}\right)-d_{k, i}\right|}{d_{k, i}}\right)\right] \quad k=\delta, v
$$

The MAPE is used to compare the accuracy of the probabilistic demand models base on 
the three different deterministic demand models (i.e., CM, N2 and MN2).

Table 3-4. Comparison of the complexity and accuracy of the developed probabilistic models

\begin{tabular}{|c|c|c|c|c|}
\hline \multirow[b]{2}{*}{ Mode } & \multirow[b]{2}{*}{$\begin{array}{l}\text { Deterministic } \\
\text { models }\end{array}$} & \multicolumn{2}{|c|}{ Model selection } & \multirow{2}{*}{$\begin{array}{c}\begin{array}{c}\text { Model } \\
\text { validation }\end{array} \\
\text { MAPE } \\
{[\%]}\end{array}$} \\
\hline & & $\begin{array}{l}\text { No. of } \\
\text { correction } \\
\text { terms }\end{array}$ & $\begin{array}{l}\text { No. of } \\
\text { intensity } \\
\text { measures }\end{array}$ & \\
\hline \multirow{3}{*}{ Deformation } & $\mathrm{CM}$ & 8 & 5 & 36.56 \\
\hline & $\mathrm{N} 2$ & 5 & 3 & 38.11 \\
\hline & MN2 & 5 & 2 & 38.10 \\
\hline \multirow{3}{*}{ Shear } & $\mathrm{CM}$ & 5 & 2 & 24.16 \\
\hline & $\mathrm{N} 2$ & 7 & 3 & 27.23 \\
\hline & MN2 & 5 & 2 & 20.58 \\
\hline
\end{tabular}

Table 3-4 shows the numbers of correction terms and of intensity measures needed to correct for the bias in each deterministic model, and the corresponding value of MAPE. The numbers of correction terms and of intensity measures represent the complexity of the model. The MAPE gauges the accuracy of the model based on the validation data. The left charts in Figure 3-6 show the deformation demand predicted based on CM, N2 and MN2 versus the corresponding values computed using the FEMs. The right charts show the mean predictions for the probabilistic models. Ideally, the predictions should line up along the 1:1 line. Predictions that systematically fall above (or below) the 1:1 line indicate that the model has in inherent bias and tends to over- (or under-) estimate the demand. The dotted lines in the right charts indicated the \pm 1 standard deviation band. Similarly, Figure 3-7 compares the predictions for the shear models. 


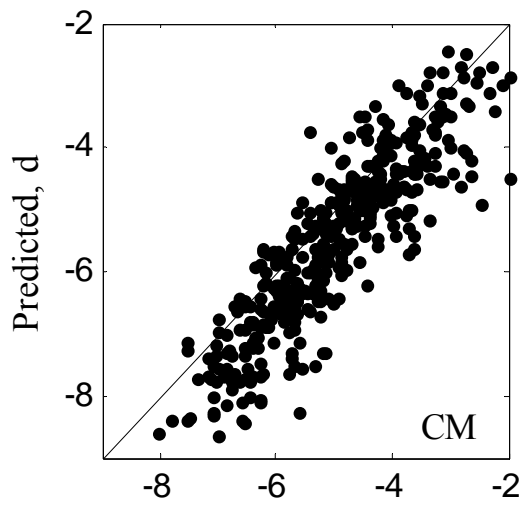

Computed using FEMs, d

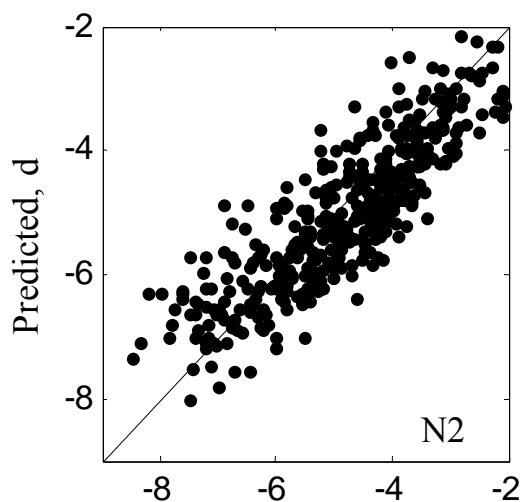

Computed using FEMs, d

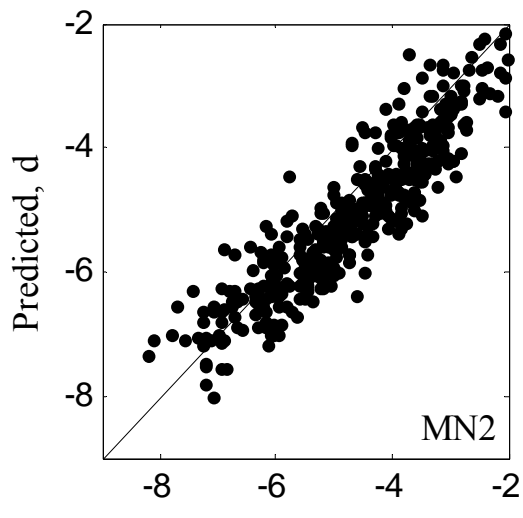

Computed using FEMs, d

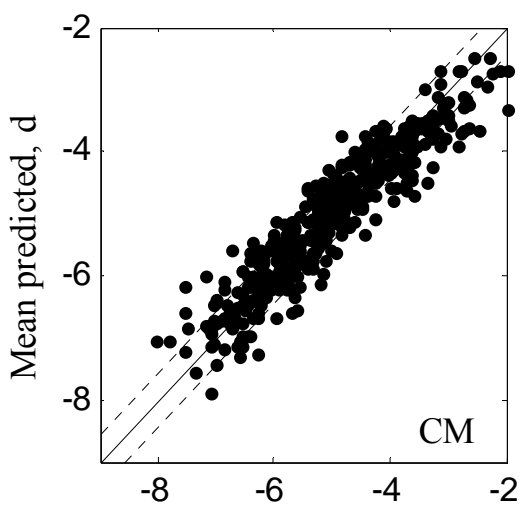

Computed using FEMs, d

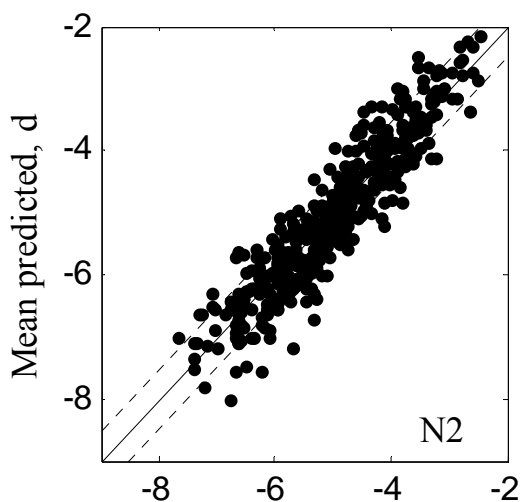

Computed using FEMs, d

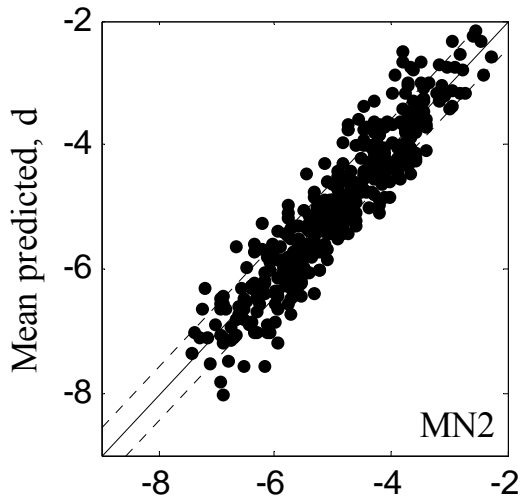

Computed using FEMs, $d$

Figure 3-6. Comparison between (logarithmic) drift ratio demand predictions based on FEMs (left) and predicted values from probabilistic demand models (right) 


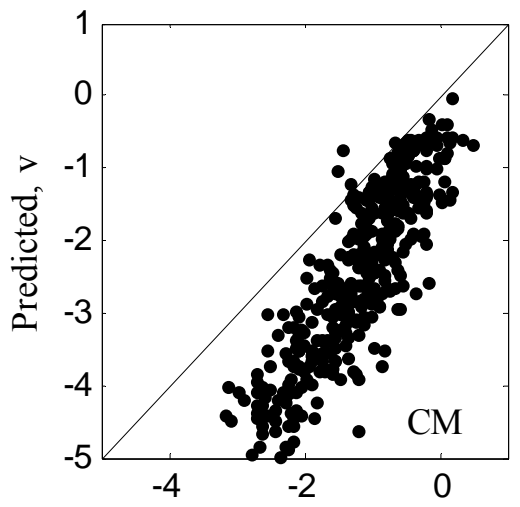

Computed using FEMs, v

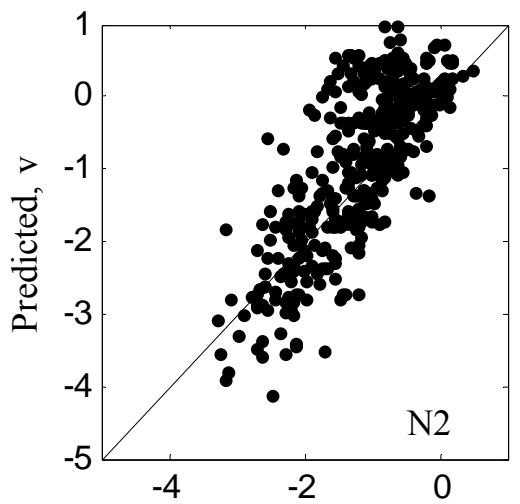

Computed using FEMs, $v$

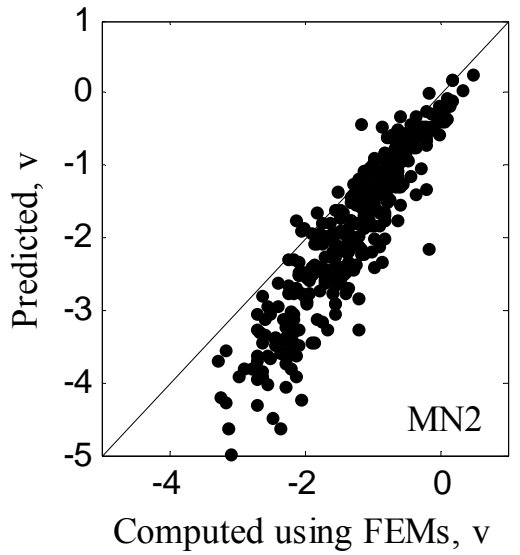

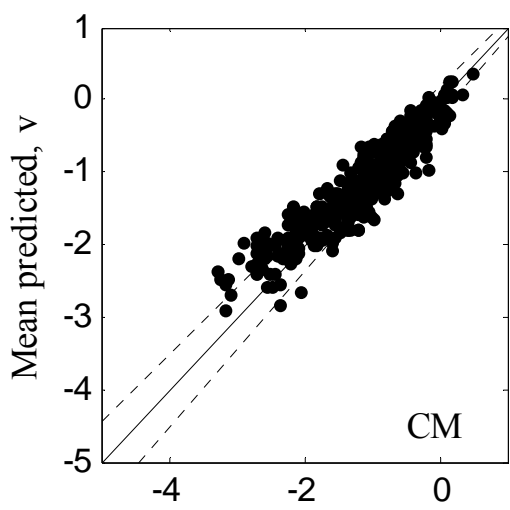

Computed using FEMs, v

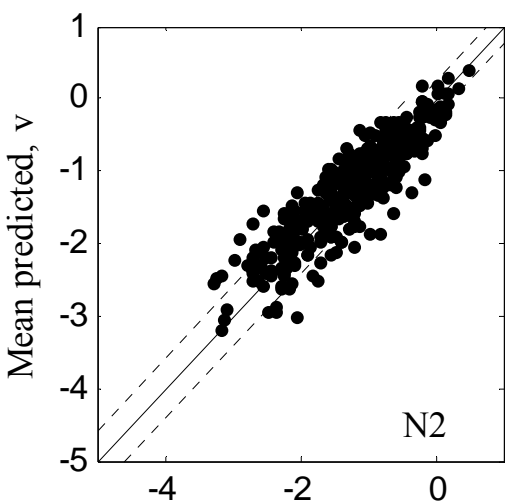

Computed using FEMs, v

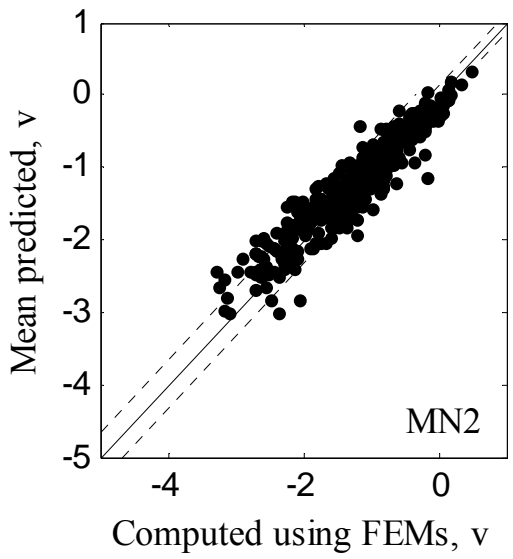

Figure 3-7. Comparison between (logarithmic) normalized shear demand predictions based on FEMs (left) and predicted values from probabilistic demand models (right)

The deformation and shear probabilistic models developed based on MN2 are 
selected because they have the best compromise between model simplicity and accuracy. The corresponding univariate deformation and shear demands are expressed as,

$$
\begin{gathered}
D_{\delta}\left(\mathbf{x}, \Theta_{D, \delta} \mid P S A, P G V\right)=\theta_{D, \delta 0}+\hat{d}_{\delta}(\mathbf{x}, P S A)+\theta_{D, \delta 1} \hat{d}_{v}(\mathbf{x}, P S A)+\theta_{D, \delta 2} \ln \left(\frac{S_{d}}{H_{C}}\right) \\
+\theta_{D, \delta 3} \ln \left(P G V \cdot \frac{T_{1}}{H_{C}}\right)+\theta_{D, \delta 4} D_{s o i l}+\theta_{D, \delta 5}\left[\hat{d}_{\delta}(\mathbf{x}, P S A)-\hat{d}_{\delta, y}(\mathbf{x})\right]_{+}+\sigma_{D, \delta} \varepsilon_{D, \delta}
\end{gathered}
$$

$$
\begin{gathered}
D_{v}\left(\mathbf{x}, \boldsymbol{\Theta}_{D, v} \mid P S A, P G V\right)=\theta_{D, v 0}+\left(1+\theta_{D, v 1}\right) \hat{d}_{v}(\mathbf{x}, P S A)+\theta_{D, v 2} \ln \left(\frac{S_{d}}{H_{C}}\right)+\theta_{D, v 3} \ln \left(P G V \cdot \frac{T_{1}}{H_{C}}\right) \\
+\theta_{D, v 4}\left[\hat{d}_{\delta}(\mathbf{x}, P S A)-\hat{d}_{\delta, y}(\mathbf{x})\right]_{-}+\theta_{D, v 5}\left[\hat{d}_{\delta}(\mathbf{x}, P S A)-\hat{d}_{\delta, y}(\mathbf{x})\right]_{+}+\sigma_{D, v} \varepsilon_{D, v}
\end{gathered}
$$

where $\sigma_{D, v}=\frac{\sigma_{v}}{\sqrt{w_{v}}}$ in which the weight $w_{v}=1.210$ if $\hat{d}_{\delta}\left(\mathbf{x}, S_{d}\right) \leq \hat{d}_{\delta, y}(\mathbf{x})$ and $w_{v}=9.0195$ if $\hat{d}_{\delta}\left(\mathbf{x}, S_{d}\right)>\hat{d}_{\delta, y}(\mathbf{x})$. The relation of $S_{d}=\left[T_{1} /(2 \pi)\right]^{2} \cdot P S A$ is used in Eq. (3.12). The two values of $w_{v}$ reflect that the model error changes going from the elastic to the inelastic state. The weight is taken as the inverse of variance of standardized residuals of the full models. Tables 3-5 and 3-6 show the posterior statistics of $\boldsymbol{\Theta}_{D, \delta}$ and $\boldsymbol{\Theta}_{D, v}$, respectively. Table 3-7 shows the posterior statistics of the model parameters $\boldsymbol{\Theta}_{D}=\left(\boldsymbol{\Theta}_{D, \delta}, \boldsymbol{\Theta}_{D, v}, \rho_{D, \delta v}\right)$ in the bivariate deformation-shear demand model, where $\rho_{D, \delta v}=$ the correlation between the model errors $\varepsilon_{D, \delta}$ and $\varepsilon_{D, v}$. 
Table 3-5. Posterior statistics of the parameters in the deformation model

\begin{tabular}{ccccccccc}
\hline \multirow{2}{*}{ Parameter } & \multirow{2}{*}{ Mean } & Standard & \multicolumn{6}{c}{ Correlation Coefficient } \\
\cline { 5 - 9 } & & Deviation & $\theta_{D, \delta 0}$ & $\theta_{D, \delta 1}$ & $\theta_{D, \delta 2}$ & $\theta_{D, \delta 3}$ & $\theta_{D, \delta 4}$ & $\theta_{D, \delta 5}$ \\
\hline$\theta_{D, \delta 0}$ & -2.796 & 0.180 & 1.00 & & & & & \\
$\theta_{D, \delta 1}$ & 0.277 & 0.044 & -0.82 & 1.00 & & & & \\
$\theta_{D, \delta 2}$ & -0.947 & 0.063 & 0.90 & 0.77 & 1.00 & & & \\
$\theta_{D, \delta 3}$ & 0.457 & 0.040 & -0.33 & 0.15 & -0.69 & 1.00 & & \\
$\theta_{D, \delta 4}$ & -0.150 & 0.036 & -0.53 & 0.49 & -0.42 & 0.10 & 1.00 & \\
$\theta_{D, \delta 5}$ & 0.542 & 0.063 & -0.83 & 0.63 & -0.65 & 0.10 & 0.32 & 1.00 \\
$\sigma_{D, \delta}$ & 0.430 & - & - & - & - & - & - & - \\
\hline
\end{tabular}

Table 3-6. Posterior statistics of the parameters in the shear model

\begin{tabular}{cccccccccc}
\hline \multirow{2}{*}{ Parameter } & \multirow{2}{*}{ Mean } & Standard & \multicolumn{7}{c}{ Correlation Coefficient } \\
\cline { 6 - 9 } & & Deviation & $\theta_{D, v 0}$ & $\theta_{D, v 1}$ & $\theta_{D, v 2}$ & $\theta_{D, v 3}$ & $\theta_{D, v 4}$ & $\theta_{D, v 5}$ \\
\hline$\theta_{D, v 0}$ & -0.551 & 0.089 & 1.00 & & & & & \\
$\theta_{D, v 1}$ & -0.107 & 0.017 & -0.30 & 1.00 & & & & \\
$\theta_{D, v 2}$ & -0.266 & 0.029 & 0.89 & -0.42 & 1.00 & & & \\
$\theta_{D, v 3}$ & 0.165 & 0.018 & -0.23 & 0.16 & -0.63 & 1.00 & & \\
$\theta_{D, v 4}$ & -0.194 & 0.023 & -0.64 & -0.31 & -0.46 & -0.05 & 1.00 & \\
$\theta_{D, v 5}$ & 0.177 & 0.026 & -0.88 & 0.37 & -0.71 & 0.07 & 0.43 & 1.00 \\
$\sigma_{v}=\sigma_{D, v} \sqrt{w_{v}}$ & 0.356 & - & - & - & - & - & - & - \\
\hline
\end{tabular}


Table 3-7. Posterior statistics of the parameters in the bivariate deformation-shear model

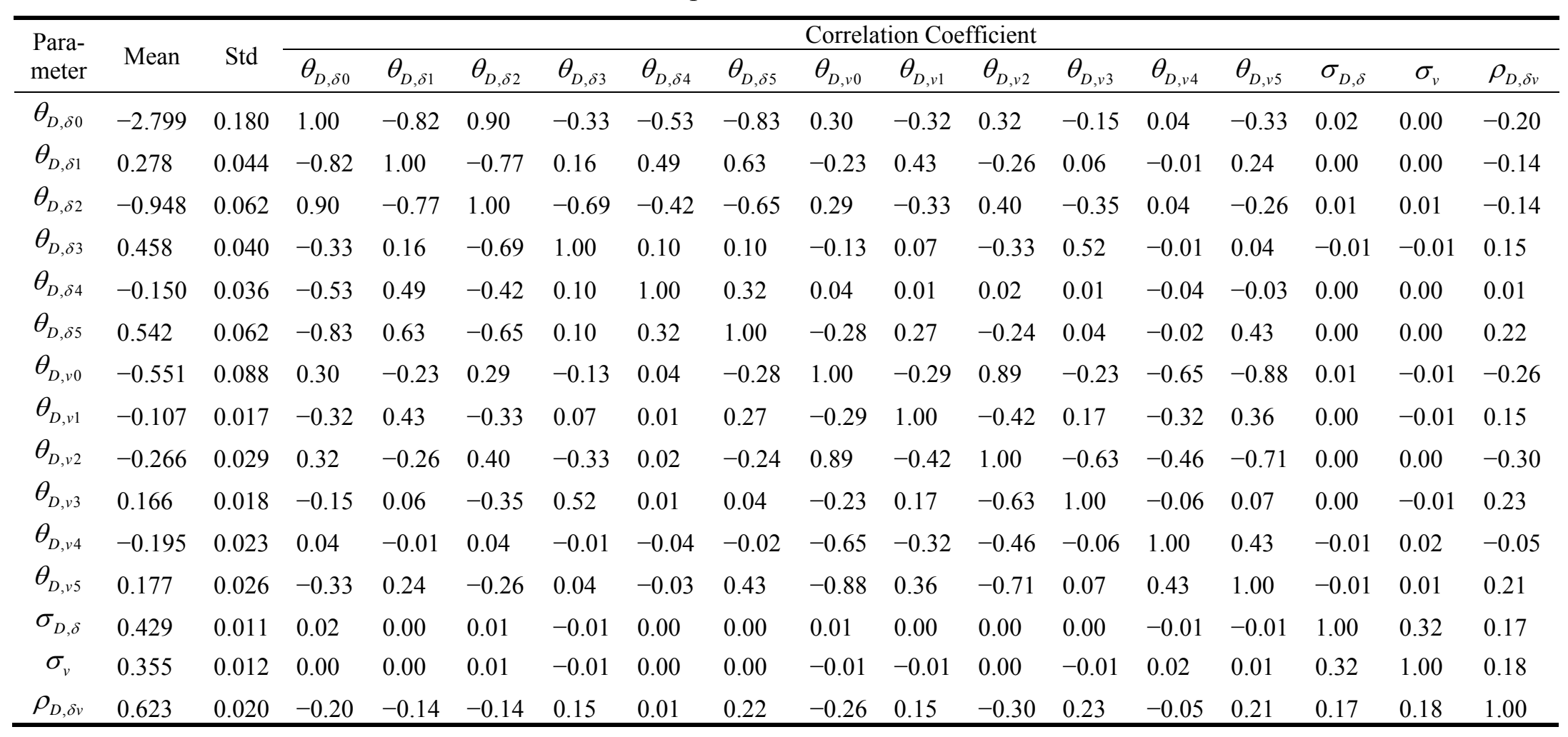




\subsubsection{Discussion of Results}

The fewer the correction terms and intensity measures needed to correct a deterministic model are, the better the deterministic model is. Among the candidate deterministic models considered in this study, MN2 is shown to be the most suitable model to use in the development of probabilistic demand models. This result indicates that using only the shear force in the column to develop the bilinear push-over curve better captures the deformation and shear behavior of bridge columns. Since the data used for developing the models are based on a class of structures, instead of a specific bridge, the conclusions drawn from such comparison are valid for the entire class of structures.

It is also observed that the standard deviations of the model errors shown in Tables 3-5 and 3-6 for the developed demand models are larger than those calculated by Gardoni et al. (2003). This is likely due to the fact that the sample size used in Gardoni et al. (2003) was not fully representative of the variability in the bridge responses. The models proposed in this study are developed using a more representative set of ground motions selected based on a bin approach. If more demand data for the same class of bridges become available in the future, the developed models can be updated and the posterior estimates of $\boldsymbol{\Theta}$ can be used as priors in the updating process.

\subsection{Application of the Demand Models to Estimate Seismic Fragilities}

Seismic fragility is defined as the conditional probability that a demand quantity attains or exceeds a specified capacity level for given values of the earthquake intensity (intensity measures). As an application of the developed probabilistic demand models, 
deformation, shear, and deformation-shear fragility estimates are developed for an example bridge. Figure 3-1 shows and Table 3-8 gives the geometry and material properties of the example bridge, where $f_{y h}=$ yielding stress of the transverse reinforcement in the column, and cover $=$ the thickness of the concrete cover in the column. To account for their inherent randomness, some quantities are considered as random variables and their statistic information is shown in Table 3-8. The deformation and shear capacities are computed using the probabilistic models in Choe et al. (2007). According to Eqs. (3.11) and (3.12), two intensity measures, PSA and PGV, are needed for computing the probabilistic demands.

Table 3-8. Design parameters for the example bridge

\begin{tabular}{cccc}
\hline Parameter & Value or Mean & COV & Distribution \\
\hline$\alpha$ & $0^{\circ}$ & - & - \\
$L_{1}$ & $30.480 \mathrm{~m}$ & $1 \%$ & Lognormal \\
$H_{c}$ & $6.706 \mathrm{~m}$ & $1 \%$ & Lognormal \\
$D_{c}$ & $1.572 \mathrm{~m}$ & $2 \%$ & Lognormal \\
$f_{y}$ & $344.738 \mathrm{MPa}$ & $5 \%$ & Lognormal \\
$f_{y h}$ & $275.790 \mathrm{MPa}$ & $5 \%$ & Lognormal \\
$f_{c}^{\prime}$ & $27.579 \mathrm{MPa}$ & $10 \%$ & Lognormal \\
$\rho_{l}$ & $3.59 \%$ & - & - \\
$\rho_{s}$ & $1.06 \%$ & - & - \\
$w_{t}$ & $45 \%$ self-weight & $25 \%$ & Normal \\
$K_{\text {soil }}$ & $(\mathrm{USGS}) \mathrm{C}$ & - & - \\
$K_{\text {abut }}$ & $\mathrm{C}$ & - & - \\
$L_{2} / L_{1}$ & 1.25 & - & - \\
cover & $0.038 \mathrm{~m}$ & $10 \%$ & Lognormal \\
\hline
\end{tabular}


In general, the uncertainty in the fragility estimate comes from the variability in the structural properties, model parameters, and model error. Following Gardoni et al. (2002), a point estimate of the fragility can be computed by replacing the model parameters with their point estimates (e.g., the posterior means). Furthermore, by ignoring both the variability in the model parameters and the structural properties, the following form for deformation and shear fragility estimates can be derived:

$$
\begin{aligned}
\hat{F}(\mathbf{s}) & =P\left[g_{k}\left(\hat{\mathbf{x}}, \hat{\boldsymbol{\Theta}}_{k}\right) \leq 0 \mid \mathbf{s}\right]=P\left[C_{k}\left(\hat{\mathbf{x}}, \hat{\boldsymbol{\Theta}}_{C, k}\right)-D_{k}\left(\hat{\mathbf{x}}, \hat{\boldsymbol{\Theta}}_{D, k}\right) \leq 0 \mid \mathbf{s}\right] \\
& =1-\Phi\left(\frac{\mu_{C, k}-\mu_{D, k}}{\sqrt{\sigma_{C, k}^{2}+\sigma_{D, k}^{2}}}\right) \quad k=\delta, v
\end{aligned}
$$

where $g_{k}(\cdot)=$ limit state function of the $k^{\text {th }}$ failure mode, $\hat{\mathbf{x}}$ and $\hat{\boldsymbol{\Theta}}_{k}=\left(\hat{\boldsymbol{\Theta}}_{C, k}, \hat{\boldsymbol{\Theta}}_{D, k}\right)$ indicate the means of $\mathbf{x}$ and $\boldsymbol{\Theta}_{k}=\left(\boldsymbol{\Theta}_{C, k}, \boldsymbol{\Theta}_{D, k}\right), \Phi(\cdot)=$ cumulative distribution function (CDF) of a standard normal random variable, $\mu_{C, k}$ and $\mu_{D, k}=$ mean estimates of the $k^{\text {th }}$ capacity and demand models, and $\sigma_{C, k}=$ standard deviation of the $k^{\text {th }}$ capacity model error.

Under the same assumptions, an approximation form for the deformation-shear fragility estimate is proposed here as

$$
\begin{aligned}
\hat{F}(\mathbf{s}) & =P\left[\bigcup_{k} g_{k}\left(\hat{\mathbf{x}}, \hat{\boldsymbol{\Theta}}_{k}\right) \leq 0 \mid \mathbf{s}\right]=P\left[\bigcup_{k} C_{k}\left(\hat{\mathbf{x}}, \hat{\boldsymbol{\Theta}}_{C, k}\right)-D_{k}\left(\hat{\mathbf{x}}, \hat{\boldsymbol{\Theta}}_{D, k}\right) \leq 0 \mid \mathbf{s}\right] \\
& =1-\Phi_{2}\left(\frac{\mu_{C, \delta}-\mu_{D, \delta}}{\sqrt{\sigma_{C, \delta}^{2}+\sigma_{D, \delta}^{2}}}, \frac{\mu_{C, v}-\mu_{D, v}}{\sqrt{\sigma_{C, v}^{2}+\sigma_{D, v}^{2}}}, \rho_{\delta v}\right)
\end{aligned}
$$


where $\Phi_{2}(\cdot)$ is the bi-variety CDF of two standard normal random variables, and $\rho_{\delta v}=$ the correlation between $g_{\delta}$ and $g_{v}$. As a further simplification, if the capacity and demand models are statistically independent, then $\rho_{\delta v}$ can be written as

$$
\rho_{\delta v}=\frac{\rho_{C, \delta v} \sigma_{C, \delta} \sigma_{C, v}+\rho_{D, \delta v} \sigma_{D, \delta} \sigma_{D, v}}{\sqrt{\left(\sigma_{C, \delta}^{2}+\sigma_{D, \delta}^{2}\right)\left(\sigma_{C, v}^{2}+\sigma_{D, v}^{2}\right)}}
$$

where $\rho_{C, \delta v}=$ correlation coefficient between the model errors of deformation capacity and shear capacity.

To check the accuracy of Eqs. (3.13) and (3.14) in accessing the fragilities of the example bridge, four different fragility estimates $\left(E 1_{k}, E 2_{k}, E 3_{k}\right.$, and $\left.E 4_{k}\right)$ for each failure mode are compared. Estimate $E 1_{k}$ is calculated based on Eq. (3.13). Estimate $E 2_{k}$ is evaluated using the same form as Eq. (3.13) but $\sigma_{D, k}^{2}$ is replaced with $\sigma_{D, k}^{2}+\sigma_{\boldsymbol{\theta}, k}^{2}+\sigma_{\mathbf{x}, k}^{2}$, where $\sigma_{\boldsymbol{\theta}, k}^{2}=$ variance in $D_{k}\left(\mathbf{x}, \boldsymbol{\Theta}_{D, k}\right)$ due to the variability in $\boldsymbol{\theta}_{D, k}$ and $\sigma_{\mathbf{x}, k}^{2}=$ variance in $D_{k}\left(\mathbf{x}, \boldsymbol{\Theta}_{D, k}\right)$ due to the variability in $\mathbf{x}$. Using Eqs. (3.11) and (3.12), $\sigma_{\boldsymbol{\theta}, k}^{2}$ can be computed in closed form, and $\sigma_{\mathbf{x}, k}^{2}$ is assessed by FOSM (first order secondmoment) that uses first order approximation with a Taylor expansion around the mean of $\mathbf{x}$. Estimate $E 3_{k}$ uses the form of Eq. (3.13) but replacing $\sigma_{D, k}^{2}$ with $\sigma_{D, k}^{2}+\sigma_{\theta, k}^{2}$ to assess the effects of only the uncertainties in $\boldsymbol{\theta}_{D, k}$ in addition to the uncertainties in the model error. Finally $E 4_{k}$ is the predictive fragility (Gardoni et al. 2002) that accounts for the uncertainties in $\boldsymbol{\theta}_{D, k}$ in an average sense. Note that $E 4_{k}$ does not make any 
assumption or approximation in accounting for the uncertainties in $\boldsymbol{\theta}_{D, k}$. The consistency of the first two estimates, $E 1_{k}$ and $E 2_{k}$, can tell us whether the variance in the model error controls the overall uncertainties in the probabilistic demand models. An agreement between $E 3_{k}$ and $E 4_{k}$ can help conclude whether the simplified form in Eq. (3.13) is a good approximation.

Figures 3-8(a) and (b) show the univariate deformation and shear fragility contour curves for the example bridge, respectively, conditioning on the normalized intensity measures $P S A$ and $P G V$. Each contour represent a fragility level between 0.1-0.9 for a given pair of $P S A$ and $P G V$. Note that the change in the variability in the shear demand model brings the discontinuities in the fragility contour lines as shown in Figure 3-8(b), because different weights are used before and after yielding. In Figures 3-8(a) and (b), the solid curves and dotted curves represent $E 1_{k}$ and $E 2_{k}$, respectively, and the dashed and dot-dashed curves correspond to $E 3_{k}$ and $E 4_{k}$. The similarities of all four curves indicate that the model error $\varepsilon_{k}$ captures most of the uncertainties in the corresponding demand model, and Eq. (3.13) is a good approximation of the fragility surface of the example bridge. Accordingly, Eq. (3.14) can be shown to be a good approximation for the bivariate deformation-shear fragility estimate shown in Figure 3-9. It is noticed that for high values of intensity measures, the interaction between the deformation and shear failure modes is more significant than at lower values. 


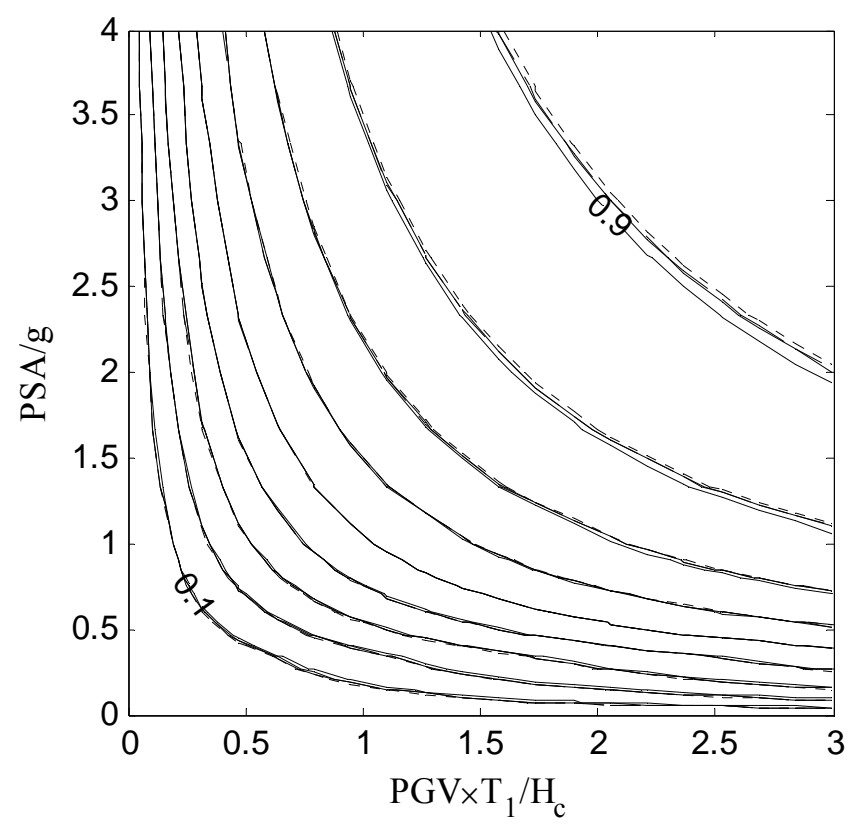

(a) Deformation fragility

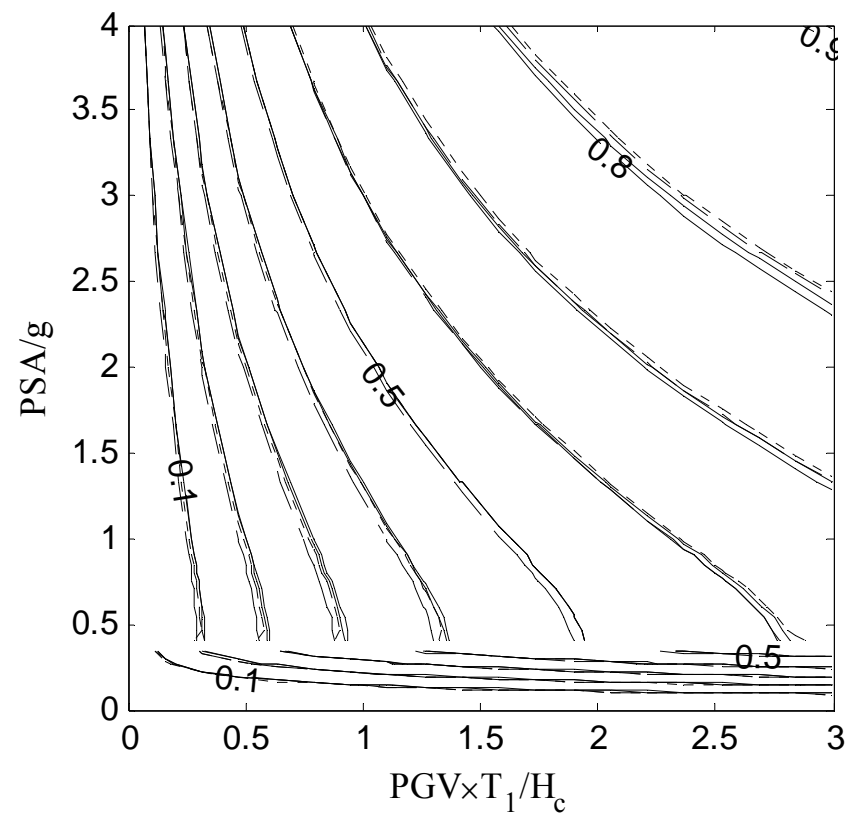

(b) Shear fragility

Figure 3-8. Univariate fragilities for the example bridge with estimates $E 1_{k}$ (solid curve), $E 2_{k}$ (dotted curve), $E 3_{k}$ (dashed curve), $E 4_{k}$ (dot-dashed curve) 


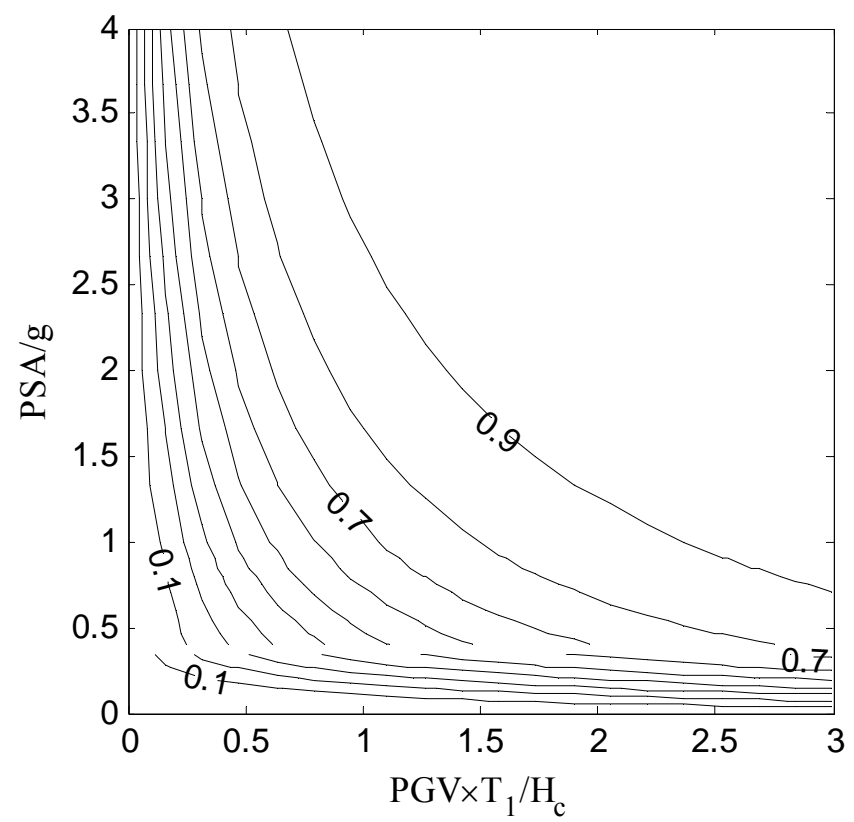

Figure 3-9. Bivariate deformation-shear fragility estimate

\subsection{Conclusions}

The study develops probabilistic models to predict the deformation and shear demands on typical RC highway bridges with one single-column bent for given ground motion intensity measures. In order to facilitate the use in practice of the developed seismic demand models, the probabilistic models are developed by adding correction terms to commonly used deterministic models to amend their inherent bias and improve the accuracy of the deterministic models. The correction terms are constructed using a set of candidate "explanatory" functions developed based on understanding of the underlying behavioral phenomena and potential ground motion parameters. Three candidate deterministic models are considered for each failure mode. A Bayesian updating approach is used to assess the unknown model parameters. The model assessment was 
conducted using virtual experimental demand data obtained from nonlinear time history analyses of detailed finite element models (FEMs) subject to a representative suite of ground motion records. The FEMs simulate RC highway bridges with one singlecolumn bent, typical of current construction in California. A computer experiment design method was used to construct 60 representative bridge configurations. An all possible subsets model selection process is used to select the explanatory functions that most effectively correct the bias and random errors in the selected deterministic demand models. As a result, the modified N2 (MN2) method developed by Gardoni et al. (2003) is shown to be the most suitable model to add in the development of probabilistic demand models.

The demand models developed in this section are based on 60 representative configurations of FEMs of RC bridges subjected to a large number of representative seismic ground motions. Therefore, the developed demand models fully consider the uncertainties associated with the structural demands on $\mathrm{RC}$ bridges due to seismic excitations. Such uncertainties include uncertainties in the ground motions and the structural properties, model errors, and statistical uncertainties in the model parameters. Note that while the FEMs are considered to be sufficient accurate, future work should quantify the model error associated to the FEMs.

As an application, the developed demand models are used to assess the univariate deformation and shear fragilities and the bivariate deformation-shear fragility for an example bridge. A simple approximate form is proposed to assess the fragilities of RC bridges. The approximate form is shown to be accurate and in good agreement with 
predictive fragility estimates. The proposed approximate form saves the computation costs associated with simulations. In general, the developed demand models and fragility estimates can be used in loss estimation and life-cycle analysis, and in determining the optimal allocation of resources for maintenance, repair, and/or rehabilitation of bridges and bridge systems. 


\section{GLOBAL NONDESTRUCTIVE TESTING}

\subsection{Introduction}

Current civil engineering infrastructure is aging and deteriorating. Damage detection is critical in evaluating the condition and performance of the existing infrastructure. Typically, visual inspections are carried out to detect potential damage. However, for large and complex structures such as bridges, visual inspection is inefficient and expensive. Furthermore, the results of visual inspection are subjective, and vary with the knowledge and experience of the inspectors. To address these issues, damage detection techniques that use vibration-based/global NDT have been increasingly studied.

Damage detection using vibration-based NDT can be classified into two categories: non-model-based methods and model-based inverse methods, as shown in Figure 4-1. Non-model-based methods, known as pattern recognition techniques, are straightforward and do not require any computer-simulated models. They have been used to detect damage location successfully (Nair et al. 2006); however, they cannot quantify the damage severity, which is critical information for evaluating the structure reliability. Additionally, a sufficient coverage on various damage scenarios is needed as training data in the non-model-based method, while usually such data obtained from the field are limited. On the other hand, model-based inverse methods require constructing a computer-simulated model but they can quantify damage severity. 


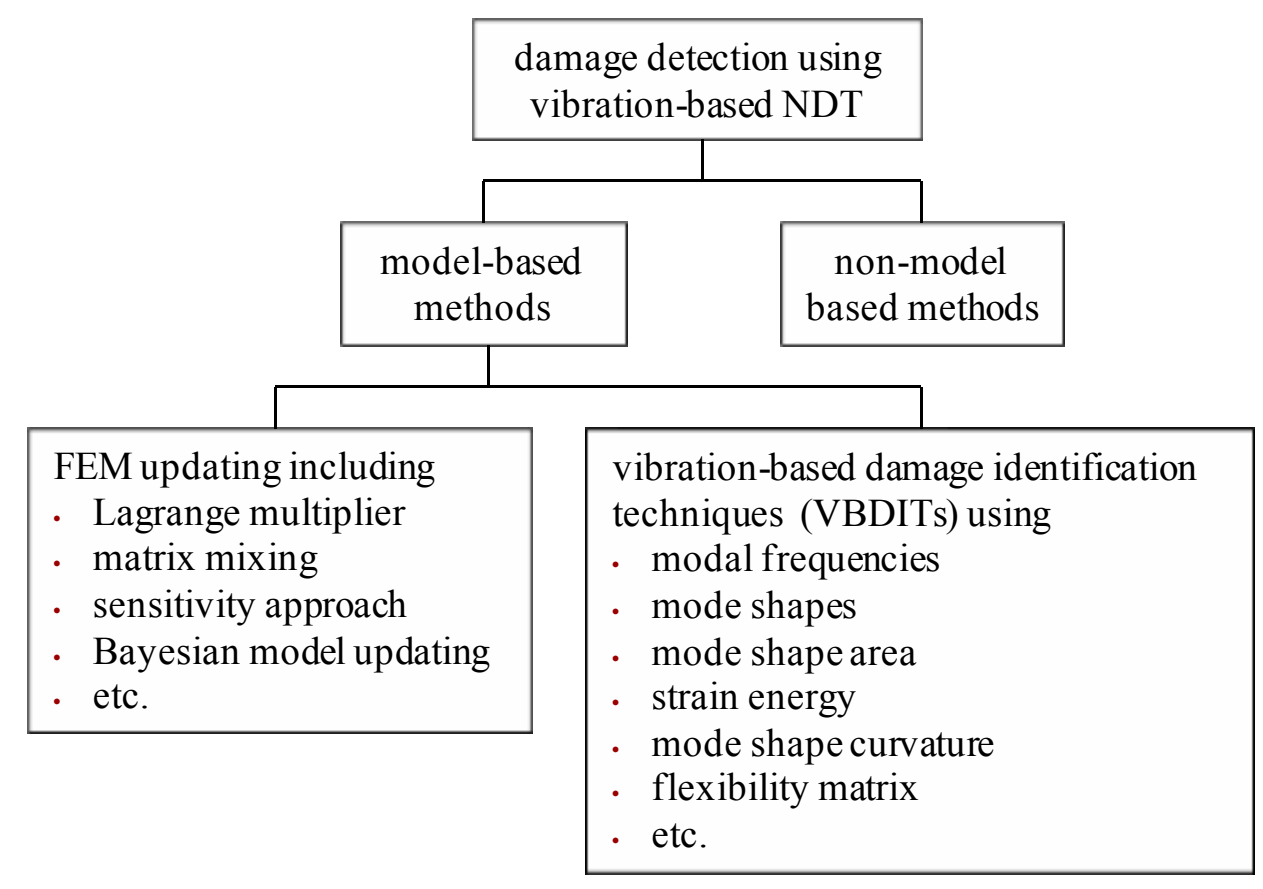

Figure 4-1. Damage detection using vibration-based NDT

Two approaches that are commonly used for model-based inverse methods are finite element model (FEM) updating and vibration-based damage identification techniques (VBDITs). Extensive reviews regarding to these two approaches can be found in Doebling et al. (1998) and Friswell and Mottershead (1995). However, damage detection using FEM updating is limited to the detection of damage at the global level due to the computational cost and potential inverse ill-conditioning problems. On the other hand, VBDITs have been used to identify local level damage successfully, but they require an accurate baseline structure that usually is not available for an existing damaged structure. Several methods have been proposed to construct a baseline such as the iterative method (Stubbs and Kim 1996), the ratio approach (Barroso and Rodriguez 
2004), and the neural network approach (Feng et al. 2004). However, these methods are deterministic and they do not account for the underlying uncertainties.

Furthermore, a damage detection method should properly treat the uncertainties present in the overall damage detection process (Sohn and Law 1997). A proper treatment of the underlying uncertainties can also help deal with the issue of illconditioning and non-uniqueness in the model inverse problem (Papadimitriou et al. 2001).

This section develops a novel probabilistic damage detection approach using modal parameters extracted from output only responses. It is proposed to use a Bayesian FEM updating not to detect damage but to construct a probabilistic baseline using modal frequencies and to use a VBDIT to detect damages using mode shapes. In this way, one can take advantage of the strength of the FEM updating that succeeds in identifying the structural properties at global level and the strength of the VBDIT in detecting the damage at local level. Following the taxonomy of uncertainties (Gardoni et al. 2002), in the proposed approach, three sources of uncertainties are taken into account in addition to the variability of the basic structural properties (geometry and material properties) of the real structure: (1) the measurement error reflecting the uncertainties in the vibration response measurements; (2) the modeling error due to the inexactness and assumptions in the modeling processes; and (3) the statistical uncertainties in the model parameters. It is important to distinguish between the different types of uncertainties because the required actions to reduce them are different. Several techniques have been proposed to 
extract modal parameters from ambient vibration data (Peerters and De Roeck 2001; Brincker et al. 2001; Kim et al. 2005), making the proposed approach of practical value.

In the following, the background and the overview of the proposed approach are firstly descibed, followed by a detailed description of the individual methods adopted in the proposed approach. Then, the sources and types of uncertainties are discussed. Next, the propagation of the uncertainties through the proposed damage detection approach is described. Finally, the proposed approach with a numerical example is illustrated.

\subsection{Proposed Damage Detection Approach}

The FEM updating determines damage as a change in the physical properties of a structure by updating the structural parameters in the FEM to match some selected quantities that are either directly measured or derived from field measurements at two different times. The selected quantities can be modal parameters (Sohn and Law 1997; Teughels et al 2002; Brownjohn et al. 2003; Jaishi and Ren 2005), time-domain response data (Koh et al 2000; Katkhuad et al 2005; Bu et al. 2006), and static responses (Banan et al. 1994a, b; Hjelmstad and Shin 1997; Sanayei et al. 2005). According to the algorithm adopted, the conventional model updating includes direct methods (such as Lagrange multiplier, matrix mixing, and Bayesian model updating) and a sensitivity approach. A review of those approaches is given in Friswell and Mottershead (1995).

Bayesian model updating has several advantages compared to other conventional model updating methods (Beck and Katafygiotis 1998): (1) it is capable of incorporating 
all types of available information, such as field observation, and engineering judgment, and all types of uncertainties, such as measurement errors, modeling errors, and statistical uncertainties; (2) it can be applied when there are only a few lower modal parameters due to a limited number of sensors in large-scale structures; (3) it does not use a sensitivity matrix that can be unstable for complex structures; and (4) the updating parameters can be continuously updated easily when new measurement data become available.

However, as mentioned previously, Bayesian model updating and in general FEM updating are limited to detect global damage due to the computational costs and potential inverse ill-conditioning problems. Therefore, one can use a Bayesian model updating to build a baseline. Note that in an undamaged (baseline) structure, each structural element has uniform stiffness throughout its length. Accordingly, the properties used to define the baseline are global level properties and a Bayesian model updating is suitable for building such a baseline.

VBDIT detects structural damage by investigating the change between the structural vibration characteristics before and after the damage occurs. The vibration characteristics include modal frequencies (Salawu 1997), mode shapes (Fox 1992; Ko et al. 1994), mode shape area (Huth et al. 2005), mode shape curvature (Pandey et al. 1991), modal strain energy (Stubbs and Kim 1996; Cornwell et al. 1997; Yu et al. 1999) and flexibility matrix (Catbas et al. 2006). In this study, with the baseline constructed by Bayesian model updating, the DIM proposed by Stubbs and Kim (1996) is adopted to detect damage using mode shapes. Among the commonly used VBDITs, the DIM has 
been shown to perform well in numerical examples (Humar et al. 2006; Alvandi and Cremona 2006) and a bridge experiment (Farrar and Jauregui 1998).

With reference to Figure 4-2, the proposed damage detection approach can be summarizes as follows:

(1) conduct a vibration test on the selected structure in the field and record the dynamic time-history responses;

(2) extract modal parameters (such as modal frequencies and mode shapes) from the time-history responses using the time domain decomposition method (TDD) (Kim et al. 2005);

(3) build a preliminary FEM based on the structure design drawings, field measurements and engineering knowledge;

(4) use the Bayesian model updating to update the preliminary FEM into a baseline by matching the extracted modal frequencies from Step (2); and

(5) identify damage using the DIM with the mode shapes of the damaged structure extracted from Step (2) and the mode shapes of the baseline built in Step (4).

The details of each step are described next.

\subsubsection{Vibration Test}

While forced vibration tests usually provide more informative data since the input can be broad-band to excite more modes, ambient vibration does not interrupt structural normal operation. Therefore, the ambient vibration is more suitable and feasible for practical applications to obtain the modal data. 


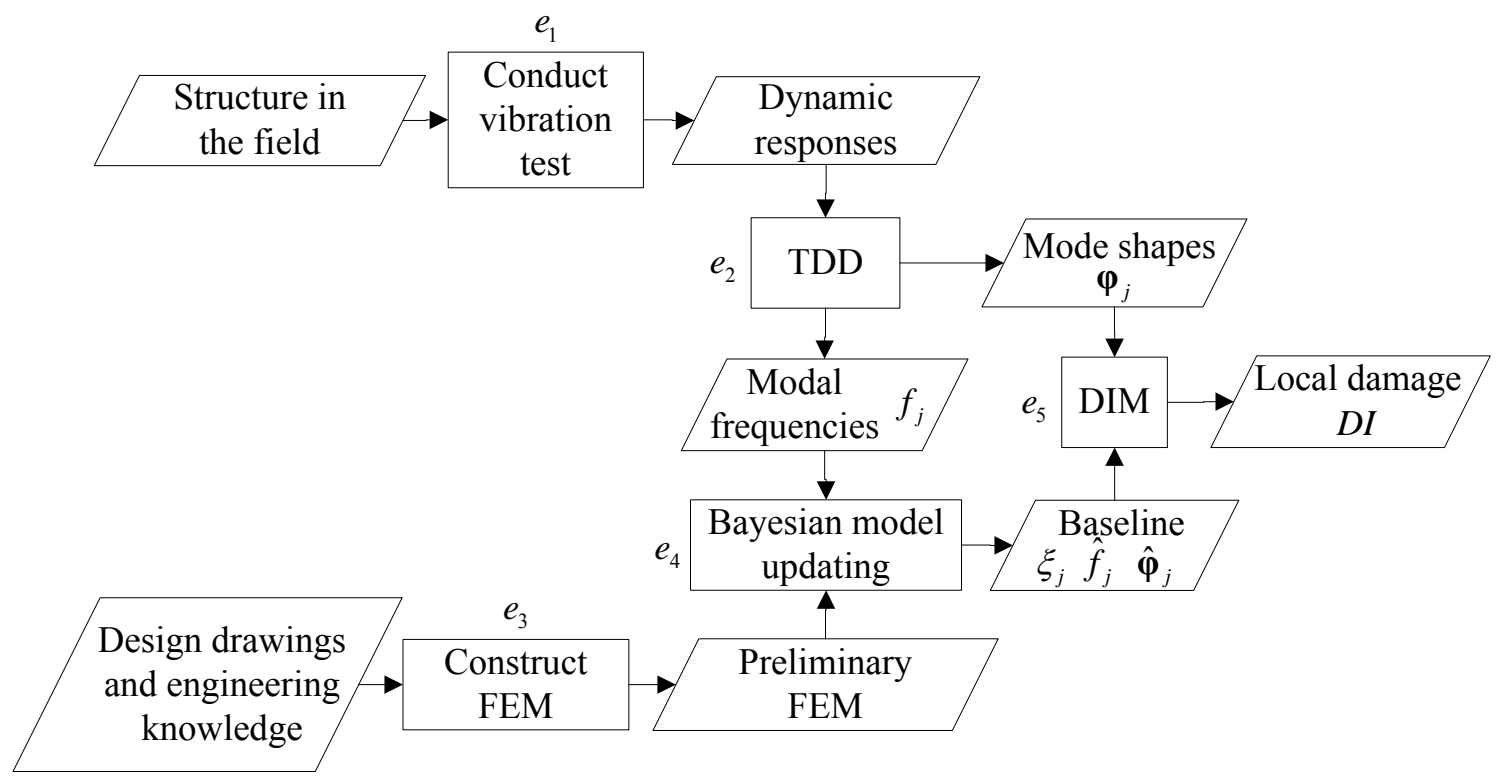

Figure 4-2. Flowchart of the proposed damage detection approach

\subsubsection{Time Domain Decomposition}

Acceleration responses are the commonly measured quantities in a vibration test. Following the modal parameter extraction method, TDD, proposed by Kim et al. (2005), if there are $n$ acceleration sensors, the output acceleration time-history can be written as

$$
\ddot{\mathbf{y}}(t)=\sum_{j=1}^{\infty} \ddot{c}_{j}(t) \boldsymbol{\varphi}_{j}=\sum_{j=1}^{n} \ddot{c}_{j}(t) \boldsymbol{\varphi}_{j}+\boldsymbol{\varepsilon}_{t}(t)
$$

where $\ddot{\mathbf{y}}(t)=\left[\ddot{\mathrm{y}}_{1}(t), \ldots, \ddot{\mathrm{y}}_{n}(t)\right], \ddot{c}_{j}(t)=j$ th mode contribution factor at time $t, \boldsymbol{\varphi}_{j}=j$ th modal shape, and $\boldsymbol{\varepsilon}_{t}(t)=\sum_{j=n+1}^{\infty} \ddot{c}_{j} \boldsymbol{\varphi}_{j}=$ truncation error. After the frequency bandwidth for each mode is estimated, a digital band-pass filter can be designed to filter out mode- 
isolated discrete-time responses. The $j$ th mode-isolated acceleration response, $\ddot{\mathbf{y}}_{j}(t)$, can be expressed as

$$
\ddot{\mathbf{y}}_{j}(t)=\ddot{c}_{j}(t) \boldsymbol{\varphi}_{j}+\sum_{k=1}^{n-1} \ddot{d}_{k}(t) \boldsymbol{\psi}_{k}=\ddot{c}_{j}(t) \boldsymbol{\varphi}_{j}+\boldsymbol{\varepsilon}_{f}(t)
$$

where $\boldsymbol{\varepsilon}_{f}(t)=\sum_{k=1}^{n-1} \ddot{d}_{k}(t) \boldsymbol{\psi}_{k}=$ error due to the truncation and the filtering, $\boldsymbol{\psi}_{k}=$ orthogonal noise base, and $\ddot{d}_{k}(t)=k$ th mode contribution factor to the total error.

By assuming orthogonal bases in the modal space that consists of $\ddot{\mathbf{c}}_{j}=\left[\ddot{c}_{j}\left(t_{1}\right), \ldots, \ddot{c}_{j}\left(t_{N}\right)\right]$ and an error space that consists of $\ddot{\mathbf{d}}_{k}=\left[\ddot{d}_{k}\left(t_{1}\right), \ldots, \ddot{d}_{k}\left(t_{N}\right)\right]$, where $N=$ total time sample, a cross-correlation $\mathbf{E}_{j}=\mathbf{Y}_{j} \mathbf{Y}_{j}^{T}$ of the $j$ th mode-isolated acceleration can be derived as

$$
\mathbf{E}_{j}=\boldsymbol{\varphi}_{j} q_{j} \boldsymbol{\varphi}_{j}^{T}+\sum_{k=1}^{n-1} \boldsymbol{\psi}_{k} \xi_{k} \boldsymbol{\psi}_{k}^{T}
$$

where $q_{j}=\ddot{\mathbf{c}}_{j}^{T} \ddot{\mathbf{c}}_{j}$ and $\xi_{k}=\ddot{\mathbf{d}}_{k}^{T} \ddot{\mathbf{d}}_{k}$. Since the energy contributed by noises is relatively small, it is appropriate to assume $q_{j}>\xi_{1}>\ldots>\xi_{n-1}$. Thus, by conducting a singular value decomposition of $\mathbf{E}_{j}$, the first singular vector in the singular vector matrix is the $j$ th mode shape $\varphi_{j}$.

With the obtained $j$ th mode shape $\boldsymbol{\varphi}_{j}$, the corresponding contribution factor of acceleration $\ddot{\mathbf{c}}_{j}$ can be calculated by

$$
\ddot{\mathbf{c}}_{j}^{T}=\frac{\boldsymbol{\varphi}_{j}^{T} \mathbf{Y}_{j}}{\boldsymbol{\varphi}_{j} \boldsymbol{\varphi}_{j}^{T}}
$$


A windowed Fast Fourier Transform (FFT) can be used to transform $\ddot{\mathbf{c}}_{j}$ into the frequency domain. The frequency at the single peak is the $j$ th modal frequency.

\subsubsection{Preliminary FEM}

Based on the design drawing of the structure of interest, field measurements, and engineering judgment, a preliminary FEM is constructed by incorporating engineering knowledge on structural mechanics, including kinematics, material laws, equilibrium, and boundary conditions. A baseline is then built by updating the preliminary FEM in the next step.

\subsubsection{Bayesian Model Updating}

Bayesian statistics (Box and Tiao 1992) enables one to use the modal parameters extracted from a real structure using TDD to update the joint PDF, $p(\mathbf{x})$, of the structural variables, $\mathbf{x}$, used in the preliminary FEM. If $\mathbf{M}$ denotes the vector of data used to update the model, the posterior PDF of $\mathbf{x}, p^{\prime}(\mathbf{x})$, can be written as

$$
p^{\prime}(\mathbf{x})=\kappa L(\mathbf{M} \mid \mathbf{x}) p(\mathbf{x})
$$

where $\kappa=\left[\int L(\mathbf{M} \mid \mathbf{x}) p(\mathbf{x}) \mathrm{d} \mathbf{x}\right]^{-1}$, and $L(\mathbf{M} \mid \mathbf{x})=$ likelihood function. Using modal frequencies and assuming that the prediction error, $\xi_{j}=\left(\hat{f}_{j}{ }^{2}-f_{j}{ }^{2}\right) / f_{j}{ }^{2}$, between the square of the frequency predicted using FEM, $\hat{f}_{j}$, and the square of the corresponding 
target frequency, $f_{j}$, follows a normal distribution, the likelihood function can be written as

$$
L(\mathbf{M} \mid \mathbf{x})=\frac{1}{\prod_{j}\left(2 \pi \cdot \sigma_{f, j}\right)} \exp \left\{\sum_{j} \frac{\left[\left(\hat{f}_{j}^{2}-f_{j}^{2}\right) / f_{j}^{2}-\mu_{f, j}\right]^{2}}{-2 \sigma_{f, j}{ }^{2}}\right\}
$$

where $\mathbf{M}=\mathrm{a}$ vector of modal frequencies, $j=$ mode number, $\mu_{f, j}$ and $\sigma_{f, j}$, respectively, refer to the mean and standard deviation of $\xi_{j}$. In other words, $\mu_{f, j}$ and $\sigma_{f, j}$, can be considered as the bias and randomness in $\xi_{j}$, respectively. The Bayesian updating is conducted using the modal frequencies because damage at local level has insignificant effects on the modal frequencies allowing constructing an accurate baseline. Furthermore, the selection of the parameters to update is critical, because a large number of updating parameters leads to high computation cost and may also result in an ill-conditioned problem. Following the suggestions in Friswell and Motterhead (1995), one should choose parameters that are most uncertain and sensitive to the vibration measurement data.

However, calculating the normalizing factor $\kappa$ can be challenging, especially when the dimension of $\mathbf{x}$ is high. To effectively compute the posterior statistics, one can use a sampling-based technique like the Markov Chain Monte Carlo (MCMC) simulations (Gilks et al. 1996). MCMC generates a sequence of random variables called Markov chain such that the current value or state of the sequence depends only on the previous value. Given certain conditions, the chain will forget its initial state and converge to a stationary distribution. For the application shown in this study, DRAM, 
which is a special form of the Metropolis-Hastings type of MCMC algorithm proposed by Haario et al. (2006) is used. DRAM incorporates the adaptive Metropolis-Hastings (AM) algorithm with the delayed rejection (DR) method, improving the efficiency of the MCMC algorithm.

\subsubsection{Markov Chain Monte Carlo (MCMC)}

MCMC, a simulation-based method, has been proposed to cope with the difficulties in obtaining the posterior distribution in Bayesian statistics updating approaches due to the requirement of the integration of high-dimensional functions. By generating a Markov chain that is introduced in the following context, MCMC can be used to estimate various characteristics of posterior distribution, such as moments, modes, and densities.

\section{Monte Carlo Integration}

Classic Monte Carlo integration draws a large sample $x_{1}, \cdots, x_{n}$ from a distribution $q(x)$, then a integral of an function $g(x)$ can be estimated by,

$$
\int_{a}^{b} g(x) d x=\int_{a}^{b} f(x) q(x) d x \approx \frac{1}{n} \sum_{i=1}^{n} f\left(x_{i}\right)
$$

As an example, to estimate the mean and variance of posterior $p^{\prime}(\mathbf{x})$ of $\mathbf{x}$, one can use the following expression,

$$
\begin{gathered}
E(\mathbf{x}) \approx \frac{1}{n} \sum_{i=1}^{n} \mathbf{x}_{i} \\
\operatorname{Var}(\mathbf{x})=\frac{1}{n} \sum_{i=1}^{n}\left[\mathbf{x}_{i}-E(\mathbf{x})\right]^{2}
\end{gathered}
$$


where $\mathbf{x}_{1}, \cdots, \mathbf{x}_{n}$ from the joint distribution $p^{\prime}(\mathbf{x})$. However, $p^{\prime}(\mathbf{x})$ is usually not known.

\section{Markov Chain}

First order Markov chain refers to a sequence of random variables, $\mathbf{X}$, from a Markov process where the current value or state of the sequence, $\mathbf{X}_{t}$ depends only to the previous one, $\mathbf{X}_{t-1}$, by drawing from a called transition kernel (or transition density) $q\left(\mathbf{X}_{t} \mid \mathbf{X}_{t-1}\right)$, where $t=1,2, \ldots$ referring as an sequence and when considering continuous states for the Markov chain, $t$ denotes the time. The Markov process denoting the process at state $s_{i}$ to state $s_{j}$ can be expressed as

$$
\operatorname{Pr}\left(\mathbf{X}_{t}=s_{j} \mid \mathbf{X}_{0}=s_{k}, \ldots, \mathbf{X}_{t-1}=s_{i}\right)=\operatorname{Pr}\left(\mathbf{X}_{t}=s_{j} \mid \mathbf{X}_{t-1}=s_{i}\right)=q(i, j)
$$

where $\operatorname{Pr}(\cdot)=$ probability. If one lets $\pi_{j}(\mathbf{X})$ represent the state $s_{j}$ at $t$, then the following equation can be obtained,

$$
\pi_{j}(t)=\sum_{\forall k} q(k, j) \cdot \pi_{k}(t-1)
$$

The above equation is called Chapman-Kolomogrov equation. The successive iteration of the Chapman-Kolomogrov equation gives the evolution of the Markov chain. In a discrete space, one can define a probability transition matrix $\mathbf{Q}$ where $q(i, j)$ is the $(i, j)$ th element. Note that the sum of each row/column of $\mathbf{Q}$ equals to one. Thus, Chapman-Kolomogrov equation can be rewritten as

$$
\boldsymbol{\pi}(t)=\boldsymbol{\pi}(t-1) \mathbf{Q}
$$

Based on the above equation, it can be shown that 


$$
\boldsymbol{\pi}(t)=\boldsymbol{\pi}(0) \mathbf{Q}^{t}
$$

where $q^{t}(i, j)$ is the $(i, j)$ th element of matrix $\mathbf{Q}^{t}$.

Furthermore, a Markov chain is called irreducible if it starts from any one of the state, there always exists a positive integer, $t$, such that it can get to any other state. A Markov chain is periodic if a state $s_{i}$ has a period, which indicates that for given that $\mathbf{X}_{t}=s_{i}$, only $\mathbf{X}_{t+n \cdot d}=s_{i}$ in which $n=$ a positive integer. A chain is said to be aperiodic when the chain is not periodic. In other words, there is no fixed integer $t$ between two certain states in an aperiodic chain. If $q(j, k) \pi_{j}=q(k, j) \pi_{k}$, then the Markov chain is called to be reversible.

It has been shown that when the Markov chain is irreducible and aperiodic, the chain will forget its initial state and the will converge to the target distribution (or stationary distribution), $\boldsymbol{\pi}^{*}$ (Robert and Casella 1999). The target distribution satisfies the following equation,

$$
\boldsymbol{\pi}^{*}=\boldsymbol{\pi}^{*} \mathbf{Q}
$$

Additionally, reversibility of a Markov chain is a sufficient condition for a unique stationary distribution.

The basic idea of discrete state of Markov chain can be extended to a continuous state, where the Chapman- Kolomogrov equation becomes,

$$
\pi_{j}(t)=\int q(k, j) \pi_{k}(t-1) \mathrm{d} k
$$

When a chain convergences to the target distribution, it satisfies

$$
\pi_{j}^{*}=\int q(k, j) \pi_{k}^{*} \mathrm{~d} k
$$


Since the samplings are not independent but have positive autocorrelation, the sample averages for estimating posterior values result in errors called Monte Carlo error. However, if $\mathbf{X}_{t}$ are drawn from the stationary distribution, the correlated sampling still can provide unbiased characteristics of the distribution when the sample size is sufficiently large.

To generate a Markov chain, a method called Delayed Rejection Adaptive Metropolis (DRAM) proposed by Haario et al. (2006) is used. DRAM is a novel combination of two ideas, adaptive Metropolis (AM) and delayed rejection (DR), for improving efficiency of MCMC algorithms. In the following, Metropolis-Hasting algorithm, AM algorithm, DR method, and then DRAM are briefly reviewed.

\section{Metropolis-Hasting Algorithm}

Metropolis-Hasting $(\mathrm{MH})$ algorithm is one of the most general algorithms used in MCMC simulation. It was used to integrate complex functions by random sampling techniques (Metropolis et al. 1953). The special cases of $\mathrm{MH}$ methods include Metropolis sampler, the independence sampler, and random-walk. The detail of this algorithm can be found in Chib and Greenberg (1995). Moreover, the target distribution $\boldsymbol{\pi}$ only needs to know up to the constant of normalization. Suppose the target distribution is the posterior distribution as shown in Eq. (4.5), then the $\mathrm{MH}$ algorithm can be formulated in the following,

i. Start with initial values of $\mathbf{x}_{0}$ as the beginning of the chain; 
ii. For each step, sample a candidate $\mathbf{x}^{*}$ from a proposal distribution $q\left(\mathbf{x}^{*}, \mathbf{x}_{t-1}\right)$ in which $\mathbf{x}_{t-1}=$ the current value of the chain;

iii. Accept the candidate $\mathbf{x}^{*}$ by the probability of a move, $\alpha$, which can be calculated by

$$
\alpha=\min \left[1, \frac{L\left(\mathbf{M} \mid \mathbf{x}^{*}\right) p\left(\mathbf{x}^{*}\right) q\left(\mathbf{x}^{*}, \mathbf{x}_{t-1}\right)}{L\left(\mathbf{M} \mid \mathbf{x}_{t-1}\right) p\left(\mathbf{x}_{t-1}\right) q\left(\mathbf{x}_{t-1}, \mathbf{x}^{*}\right)}\right]
$$

When $\alpha \geq 1, \mathbf{x}^{*}$ are accepted unconditionally. Notice that the normalizing factor $\kappa$ in Eq. (4.5) canceled out, which voids the complexity for estimating $\kappa$.

iv. If $\mathbf{x}^{*}$ is rejected, then set $\mathbf{x}_{t}=\mathbf{x}_{t-1}$ and go back to step (ii) until enough samplings are generated.

The special cases of MH methods include Metropolis sampler, the independence sampler, and random-walk. The Metropolis sampler uses $q\left(\mathbf{x}^{*}, \mathbf{x}_{t-1}\right)=q\left(\mathbf{x}_{t-1}, \mathbf{x}^{*}\right)$; independence sampler uses such proposal distribution that $q\left(\mathbf{x}^{*}, \mathbf{x}_{t-1}\right)=q\left(\mathbf{x}^{*}\right)$; and random-walk Metropolis adopt proposal distribution $q\left(\mathbf{x}^{*}, \mathbf{x}_{t-1}\right)=q\left(\left|\mathbf{x}^{*}-\mathbf{x}_{t-1}\right|\right)$.

\section{Adaptive Metropolis Algorithm}

The proposal distribution, $q\left(\mathbf{x}^{*}, \mathbf{x}_{t-1}\right)$ used in $\mathrm{MH}$ methods play a critical role in the convergence rate of simulation. The closer the proposal distribution gets to the target distribution, the faster the convergence and the better the acceptance rate. An adaptive version of the random walk Metropolis-Hastings algorithm developed by Haario et al. (2001) uses the values in the chain generated so far to construct covariance matrix of the 
proposal distribution. That is after an initial non-adaptation period $\left(t>t_{0}\right)$, the proposal distribution is centered at the current state of the sequence, $\mathbf{x}_{t}$, with adaptive covariance $\Sigma_{q}=s_{d} \operatorname{Cov}\left(\mathbf{x}_{0}, \ldots, \mathbf{x}_{t-1}\right)+s_{d} \varepsilon \cdot I_{d}$, where $s_{d}=$ a scale parameter that depends only on the dimension, $d$, of the state space $\mathbf{x}$ and it is taken as $2.4^{2} / d$ suggested by Gelman et al. (1996), $\varepsilon=$ a non-negative and small constant that ensure $\boldsymbol{\Sigma}_{q}$ will not be singular, and $I_{d}=$ an identity matrix with dimension $d$. The procedure of AM can be summarized as below:

i. Set values for $t_{0}, s_{d}$, and $\varepsilon$, and start with initial values of $\mathbf{x}_{0}$ as the beginning of the chain and initial proposal covariance matrix $\boldsymbol{\Sigma}_{q}=\boldsymbol{\Sigma}_{q, 0}$;

ii. For each step, sample a candidate $\mathbf{x}^{*}$ from a proposal distribution $q\left(\mathbf{x}^{*}, \mathbf{x}_{t-1}\right)$ with covariance matrix $\Sigma_{q}$, in which $\mathbf{x}_{t-1}=$ the current value of the chain;

iii. Accept the candidate $\mathbf{x}^{*}$ by the probability of a move, $\alpha$, which can be calculated by Eq. (4.17);

iv. If $\mathbf{x}^{*}$ is rejected, then set $\mathbf{x}_{t}=\mathbf{x}_{t-1}$ and go back to step ii until enough samplings are generated;

v. For $t>t_{0}$, the proposal covariance matrix will be adapted by $\Sigma_{q}=s_{d} \operatorname{Cov}\left(\mathbf{x}_{0}, \ldots, \mathbf{x}_{t-1}\right)+s_{d} \varepsilon \cdot I_{d}$.

\section{Delayed Rejection Method}

DR developed by Mira (2001) provides a local adaptive strategy for the rejected values 
in a Markov chain. Alternatively, a second (or higher) stage move proposed for the rejection point instead retaining the same sampling for the next state, while the Markov chain properties and reversibility are remained. If the acceptance probability shown in Eq. (4.17) is called as the first stage acceptance probability $\alpha_{1}\left(\mathbf{x}_{t-1}, \mathbf{x}^{*}\right)$, then the second stage acceptance probability can be expressed by

$$
\alpha_{2}\left(\mathbf{x}_{t-1}, \mathbf{x}^{*}, \mathbf{x}^{* *}\right)=\min \left\{1, \frac{L\left(\mathbf{D} \mid \mathbf{x}^{*}\right) p\left(\mathbf{x}^{*}\right) q\left(\mathbf{x}^{* *}, \mathbf{x}^{*}\right) q\left(\mathbf{x}^{* *}, \mathbf{x}^{*}, \mathbf{x}_{t-1}\right)\left[1-\alpha_{1}\left(\mathbf{x}^{*}, \mathbf{x}^{* *}\right)\right]}{L\left(\mathbf{D} \mid \mathbf{x}_{t-1}\right) p\left(\mathbf{x}_{t-1}\right) q\left(\mathbf{x}_{t-1}, \mathbf{x}^{*}\right) q\left(\mathbf{x}_{t-1}, \mathbf{x}^{*}, \mathbf{x}^{* *}\right)\left[1-\alpha_{1}\left(\mathbf{x}, \mathbf{x}^{*}\right)\right]}\right\}
$$

The higher stage can be written in the same fashion as for the second stage. The covariance $\boldsymbol{\Sigma}_{q}^{(i)}=\gamma_{i} \boldsymbol{\Sigma}_{q}^{(1)}$ of the $i$ th stage can be chosen as scaled version of the proposal of the first stage, where $\gamma_{i}=$ scaling factor, $i=1, \ldots, N_{\text {try }}$ in which $N_{\text {try }}$ is the number of the tries allowed. Because of the reversibility of the chain, DR will leads to the same stationary distribution.

\section{Delayed Rejection Adaptive Metropolis}

DRAM is a combination of two algorithms: DR and AM, proposed by Haario et al. (2006). This idea considers AM as a global adaption procedure and uses DR as a local adaption for the rejected values from AM. Through adaption, different proposal distributions make it possible to provide better results. The procedure of DRAM can be described briefly as below: 
i. Set values for $t_{0}, s_{d}, \varepsilon, \gamma_{i}$, and.$N_{t r y}$., and start with initial values of $\mathbf{x}_{0}$ as the beginning of the chain and initial proposal covariance matrix $\boldsymbol{\Sigma}_{q}^{(1)}=\boldsymbol{\Sigma}_{q, 0}$;

ii. For each step, sample a candidate $\mathbf{x}^{*}$ from a proposal distribution $q\left(\mathbf{x}^{*}, \mathbf{x}_{t-1}\right)$ with covariance matrix $\boldsymbol{\Sigma}_{q}^{(1)}$, in which $\mathbf{x}_{t-1}=$ the current value of the chain;

iii. Accept the candidate $\mathbf{x}^{*}$ by the probability of a move, $\alpha$, which can be calculated by Eq. (4.17);

iv. If $\mathbf{x}^{*}$ is rejected, then go to a DR loop where maximum . $N_{t r y}$. tries will be made, where propose $\mathbf{x}^{* *}$ from a proposal distribution $q\left(\mathbf{x}^{*}, \mathbf{x}_{t-1}\right)$ with covariance matrix $\Sigma_{q}^{(k)}$ and accept the propose value with $k$ th stage of acceptance probability.

v. For $t>t_{0}$, the proposal covariance matrix will be adapted by $\Sigma_{q}^{(1)}=s_{d} \operatorname{Cov}\left(\mathbf{x}_{0}, \ldots, \mathbf{x}_{t-1}\right)+s_{d} \varepsilon \cdot I_{d}$.

vi. Go back to step ii until enough samplings are generated;

After the MCMC run using DRAM, the chain can be used as a sample from the posterior distribution $p^{\prime}(\mathbf{x})$ in Eq. (4.5). Then, Monte Carlo integration as shown in Eq. (4.7) can be used for assessing the posterior statistics. In this study, the simulated annealing method to the Markov chain (Van and Arts 1987) is applied to obtain a rapidly mixing sampler. For the convergence criteria, the Geweke method (Geweke 1992) is used in this study, which provides unbiased estimates of the posterior statistics. 


\subsubsection{Damage Index Method}

The details of DIM are described in Section 1.

\subsection{Uncertainties in the Proposed Damage Detection Approach}

As described previously, the proposed approach consists of five steps: conducting ambient vibration tests, extracting modal parameters using TDD, constructing a preliminary FEM, building a baseline, and applying the DIM. Each of these steps has associated errors that need to be recognized and accounted for. Due to those errors, the damage detection results are not deterministic anymore and one can talk about the probability that the proposed method identifies damage or not. The errors for each of the five steps can be described as follows. Table 4-1 provides additional details on the description of the errors and their influencing factors. The names used to describe the errors are based on the taxonomy in Gardoni et al. (2002).

Conducting ambient vibration tests: The errors associated with ambient vibration tests are mainly due to noises in the signals and the data processing. These errors are called measurement errors and indicated as $e_{1}$. They are epistemic in nature and can be reduced by using more accurate sensors and improving the data processing. Accounting for the effect due to the measurement error in the vibration test is described later.

Extracting modal parameters using TDD: The errors are associated to this step primarily due to the mode isolation process. In a real structure, modes overlap due to the presence of damping. TDD uses digital bandwidth filter to isolate the individual 
modes but it cannot completely filter out the influence of other modes. Other sources of error are the truncation of non-dominant models and numerical errors in the singular value decomposition. These errors are called modeling errors and indicated as $e_{2}$. They are also epistemic errors and they can be reduced by improving the process used to extract the modal parameters. Later, it gives the details of estimating the modeling errors associated with TDD. To estimate these errors can be critical for the success of damage detection.

Constructing a preliminary FEM: The errors present in this step refer to the difference between the predicted response in the FEM and the response from the real structure. They are called modeling errors and denoted as $e_{3}$. These errors can be considered as two parts: $e_{3 a}$ and $e_{3 b}$. Errors $e_{3 a}$ mainly come from the uncertainties in geometries and material properties, and influence of the environmental variability on the structural response. The nature of $e_{3 a}$ is both epistemic and aleatory. The epistemic part can be reduced in the model updating by calibrating the FEM with measurement data. The sources of $e_{3 b}$ include the inaccuracy of the model forms (such as material models), the assumptions used (such as the assumption related to the boundary conditions), and numerical errors (such as the discretization by approximate the shape functions). The errors $e_{3 b}$ are epistemic in nature and can be reduced by improving the accuracy and sophistication of the FEM. Haukaas and Gardoni (2009) proposed a general methodology and specific implementations to consider $e_{3 b}$. 
Table 4-1. Errors and uncertainties in the proposed damage detection approach

\begin{tabular}{|c|c|c|c|c|}
\hline \multirow{2}{*}{ Process } & \multirow{2}{*}{ Method } & \multicolumn{3}{|c|}{ Error } \\
\hline & & Symbol & $\begin{array}{l}\text { Description } \\
\end{array}$ & Influencing factors \\
\hline $\begin{array}{l}\text { Conducting ambient } \\
\text { vibration tests (1) }\end{array}$ & Record & $e_{1}$ & $\begin{array}{ll}\text { - } & \text { electromagnetic noises in sensor; signal conditioning } \\
\text { - } & \text { data acquisition; discretization } \\
\text { - } & \text { data processing and testing economy } \\
\text { - } & \text { system nonlinearity }\end{array}$ & $\begin{array}{ll}\text { - } & \text { sensor type; signal conditioning type } \\
\text { - } & \text { data-acquisition system; sampling rate } \\
\text { - } & \text { signal processing system } \\
\text { - } & \text { instrumentation; excitation }\end{array}$ \\
\hline $\begin{array}{l}\text { Extracting modal } \\
\text { data from dynamic } \\
\text { responses }(2)\end{array}$ & TDD & $e_{2}$ & $\begin{array}{ll}- & \text { mode isolation process } \\
- & \text { truncating non-dominant modes } \\
\text { - } & \text { singular value decomposition } \\
\end{array}$ & $\begin{array}{ll}\text { - } & \text { filter width; response length } \\
\text { - } & \text { excitation type; sensor layout } \\
\text { - } & \text { noise orthogonal assumption } \\
\end{array}$ \\
\hline & & $e_{3 a}$ & $\begin{array}{ll}\text { - } & \text { environmental variability } \\
\text { - } & \text { uncertainties in geometries and material properties }\end{array}$ & $\begin{array}{l}\text { - } \quad \text { temperature and humidity } \\
\text { - } \quad \text { engineering knowledge }\end{array}$ \\
\hline $\begin{array}{l}\text { Constructing } \\
\text { preliminary FEM } \\
\text { (3) }\end{array}$ & $\begin{array}{l}\text { Engineer } \\
\text { knowledge }\end{array}$ & $e_{3 b}$ & $\begin{array}{ll}\text { - } & \text { non- or structural members not accounted in FEM } \\
\text { - } & \text { modeling of damping } \\
\text { - } & \text { material models } \\
\text { - } & \text { idealization of loads and boundary conditions } \\
\text { - } & \text { weak form of the boundary value problems } \\
\text { - } & \text { discretization of approximate shape functions } \\
\text { - } & \text { numerical integration of element integrals } \\
\text { - } & \text { iterative scheme to attain equilibrium } \\
\text { - } & \text { explicit or implicit time-stepping schemes }\end{array}$ & $\begin{array}{ll}\text { - } & \text { engineering knowledge } \\
\text { - } & \text { engineering knowledge } \\
\text { - } & \text { engineering knowledge } \\
\text { - } & \text { engineering knowledge } \\
\text { - } & \text { mathematical formulation } \\
\text { - } & \text { mathematical formulation } \\
\text { - } & \text { mathematical formulation } \\
\text { - } & \text { mathematical calculation } \\
\text { - } & \text { mathematical calculation } \\
\text { - } & \text { mathematical calculation }\end{array}$ \\
\hline \multirow{2}{*}{$\begin{array}{l}\text { Updating } \\
\text { preliminary FEM } \\
\text { using modal data (4) }\end{array}$} & \multirow{2}{*}{$\begin{array}{l}\text { Bayesian } \\
\text { Model } \\
\text { Updating }\end{array}$} & $e_{4 a}$ & - objective function & - formulation of likelihood function \\
\hline & & $e_{4 b}$ & - $\quad$ sampling process & - $\quad$ sampling size \\
\hline $\begin{array}{l}\text { Detecting local } \\
\text { damage using } \\
\text { baseline and } \\
\text { damaged structure } \\
\text { modal shapes }(5)\end{array}$ & DIM & $e_{5}$ & $\begin{array}{l}\text { - } \text { relation between the index and the damage } \\
\text { - } \text { mode shape expansion } \\
\text { calculating of damage index }\end{array}$ & $\begin{array}{ll}\text { - } & \text { threshold } \\
\text { - } & \text { expansion algorithm } \\
\text { - } & \text { assumption }\end{array}$ \\
\hline
\end{tabular}


Building a baseline FEM: The errors in this step are due to two sources: (1) the formulation of the likelihood function in the Bayesian updating that relays on an the assumption that the prediction errors follows a normal distribution, and (2) the sampling size when MCMC is used to estimate the posterior statistics of the structural parameters. The error from the first source is a form of modeling errors and is indicated as $e_{4 a}$. The error from the second source is called statistical uncertainty and is indicated as $e_{4 b}$. Both $e_{4 a}$ and $e_{4 b}$ represent epistemic uncertainties and they can be reduced. The errors $e_{4 a}$ can be reduced by using appropriate variance stabilizing transformations (Box and Cox 1964) and $e_{4 b}$ can be reduced by increasing the sample size.

Applying the DIM: The errors present in the DIM are the errors between the DI and the damage itself, which strongly depend on the threshold value selected. These errors are also called modeling errors and indicated as $e_{5}$. They are epistemic and can be reduced by choosing an appropriate threshold value. When one chooses a threshold $\lambda_{i}$, the probabilities that the DIM indicates damage $\left(I D_{i}\right)$ and does not indicating damage $\left(N I D_{i}\right)$ in a component $i$ can be defined as,

$$
\begin{gathered}
P\left(I D_{i}\right)=P\left(Z_{i} \geq \lambda_{i}\right) \\
P\left(N I D_{i}\right)=P\left(Z_{i}<\lambda_{i}\right)
\end{gathered}
$$

Four cases can happen for a given threshold. If there exists damage in the $i$ th segment, the probability of correctly detecting this damage is denoted as $P\left(I D_{i} \mid D\right)$, and the probability of not detecting the damage is expressed as $P\left(N I D_{i} \mid D\right)$. If there is no 
damage (event $N D$ ) in the $i$ th component, the probability of correct detection is written as $P\left(N I D_{i} \mid N D\right)$ and the probability of false detection is $P\left(I D_{i} \mid N D\right)$. Ideally, one wants to choose an appropriate threshold to maximize $P\left(I D_{i} \mid D\right)$ and $P\left(N I D_{i} \mid N D\right)$, and to minimize $P\left(N I D_{i} \mid D\right)$ and $P\left(I D_{i} \mid N D\right)$. As shown in Figure 4-3, lowering the threshold can increase $P(I D \mid D)$, but it also increases $P(I D \mid N D)$. If the threshold is set too high, a high probability of missing a damage $P(N I D \mid D)$ is obtained, although $P(N I D \mid N D)$ also increases.
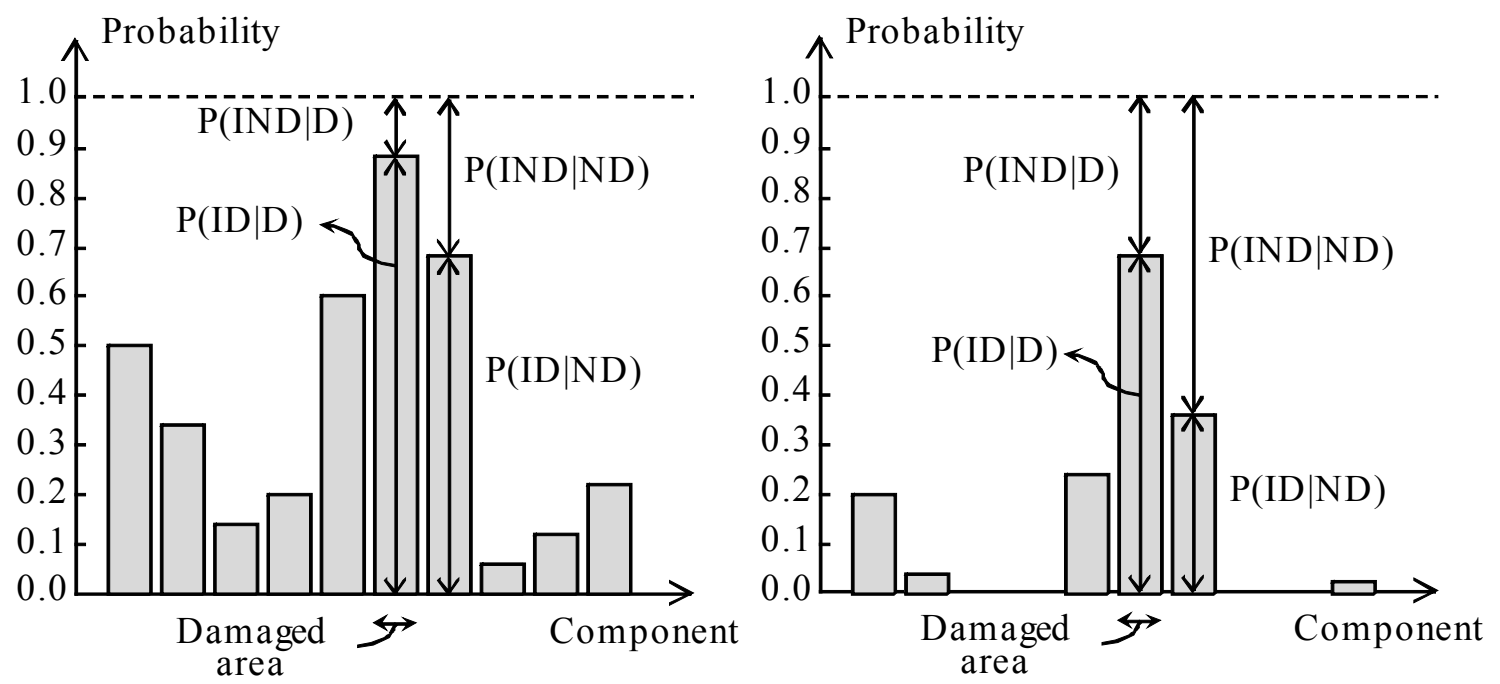

Figure 4-3. Conceptual illustration of correct and false detection probabilities when a low threshold (left) and a high threshold (right) are used

\subsection{Propagation of Uncertainties}

The errors described in the previous section propagate through the proposed damage detection approach as shown in Figure 4-2. In particular, the modal frequencies $f_{j}$ and 
the mode shapes $\boldsymbol{\varphi}_{j}$ of the damaged structure are affected by $e_{1}$ and $e_{2}$ because they are extracted by TDD from the ambient vibration response records. The prediction error $\xi_{j}$, the modal frequencies $\hat{f}_{j}$ and the mode shapes $\hat{\boldsymbol{\varphi}}_{j}$ of the baseline are affected by $e_{1}, e_{2}$, $e_{3}$ and $e_{4}$ because they obtained by calibration the preliminary FEM using the Bayesian updating based on $f_{j}$. Finally, the $D I$ is influenced by $e_{1}, e_{2}, e_{3}, e_{4}$ and $e_{5}$.

Note that modal parameters have been commonly used in system identification to validate and/or update a computer simulated model (Doebling and Farrar 2001). They are also adopted in the vibration-based NDT for damage detection such as the study of this section, where the modal parameters obtained form a damaged system with those in the corresponding undamaged system are compared. However, studies in assessing uncertainties in modal parameters are still limited, especially in the area of considering the modeling error due to the modal parameter extraction process. A typical approach is to assume that modal parameters consist of deterministic quantities and additive random errors with zero mean (Liu 1995; Papadopoulos and Garcia 1998; Xia et al. 2002; Pothisiri and Hjelmstad 2003). In this approach, regardless of the inaccuracy of this assumption, the contributions from the measurement and the extracting processes are obscured, which is not able to provide guidance of allocating recourses to improve the estimation of modal data.

In the following subsections, general procedures to assess the variability of modal data due to $e_{1}$ and $e_{2}$ are presented. A bootstrap approach is used to propagate $e_{1}$ and $e_{2}$ to the modal data, when an ensemble of a limited number of noised time-history 
response recordings is available. To estimate the modeling error associated with the extraction process, we adopted a model prediction expansion approach where the modeling error is considered as an "adjustment" directly to the prediction obtained from the modal data extraction process. The modeling error considered in this study is due to a specific modal data extraction process, TDD (Kim et al. 2005). The proposed procedures can be used to account for the influence of $e_{1}$ and $e_{2}$ when the modal parameters are furthered applied to system identification, damage detection, and other applications.

\subsubsection{Measurement Errors in Ambient Vibration Test}

The measurement error reflects the difference between the measured responses and the true responses. It may vary with sensor type, layout, installation of equipment, and environment. In numerical studies, the measurement error is generally modeled by adding random errors into the time-history response signals and the error is often simulated as a random Gaussian noise with zero mean and a specific standard deviation. To provide reasonable bounds on the measurement error, absolute error and proportional error are commonly used (Banan et al. 1994b). For absolute error, the standard deviation of measurement error is a fixed value; while for proportional error, the standard deviation of the measurement error is proportional to the value of the amplitude of the response. 


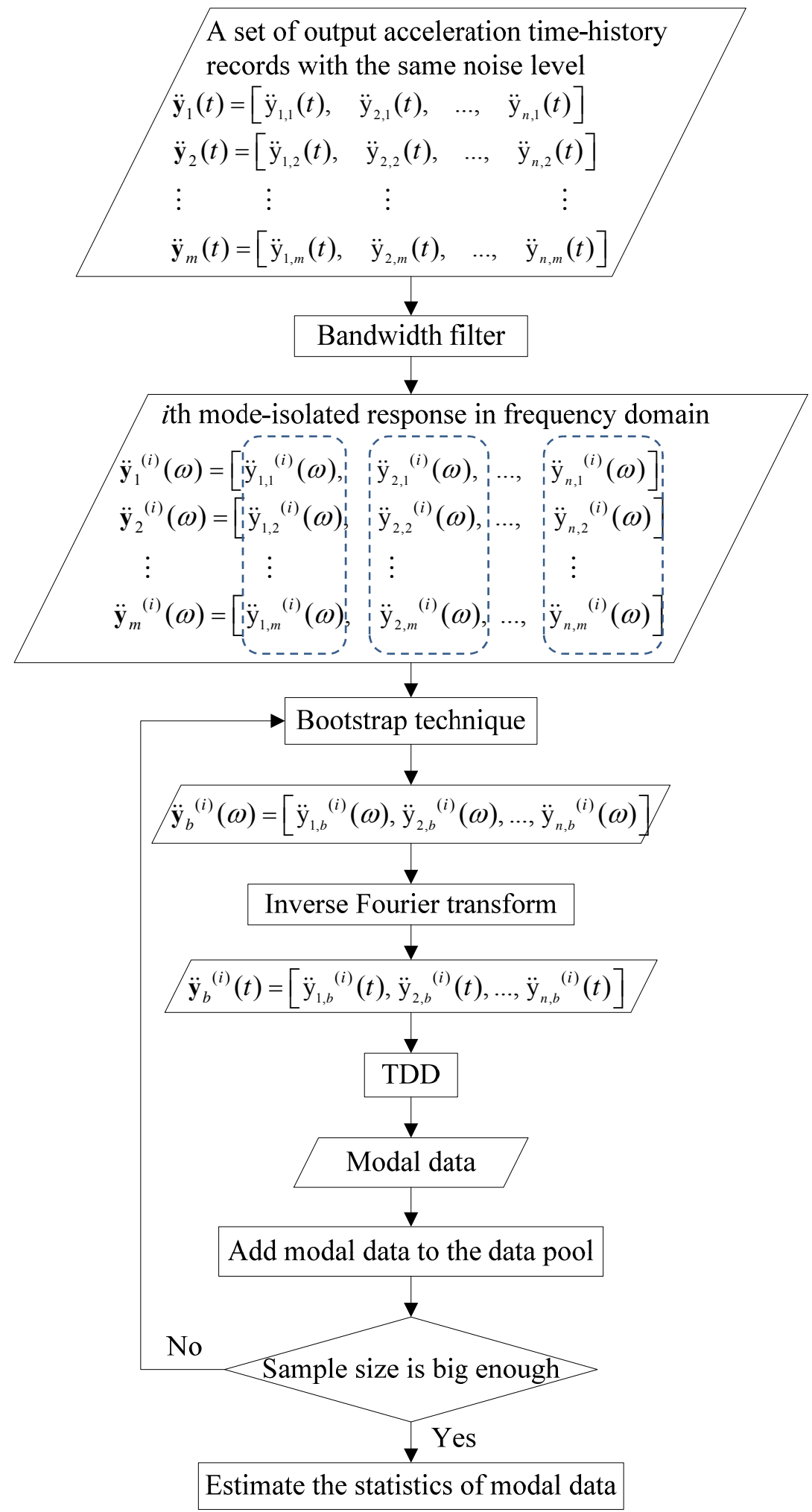

Figure 4-4. Flowchart of the procedures to propagate the measurement noises to the modal data 
Two typical ways are used to propagate the measurement error to modal data. One is the perturbation method or sensitivity method, which requires finding the sensitivity of the measurement error to the modal data. In this respect, Longman and Jung (1987) developed a framework for eigenrealization algorithm (ERA); Peterson et al. (1996) used the perturbation analysis for fast ERA; and Arici and Mosalam (2005) provided a formulation of sensitivity approach for observer Kalman filter identificationERA with direction correlations. Although the perturbation approach is straightforward and efficient after the sensitivity is expressed analytically, the procedure and computation of obtaining the sensitivity is not an easy task, and the first order approximation that may not be accurate is usually used in the sensitivity analysis (Doebling and Farrar 2001). The other approach of propagating the measurement error into the modal parameters is the sampling method. The sampling method is conducted by extracting modal data from a set of time-history responses that are contaminated by measurement error. Note that the variability in the modal parameters reflects the effect from the measurement error and the modeling error associated with the modal data extraction process. The sampling method is straightforward but requires a good number of time-history responses.

Usually, a certain number of dynamic time-history responses with a particular noise level can be recorded. For each series of the responses, TDD is applied to obtain one set of modal data. Thus the statistical inferences of the modal data can be estimated. With a limited number of dynamic response series, following Efron (1982), a bootstrap method is used to generate more records at the same measurement noise level to 
propagate the measurement noises to the modal data. In an ambient vibration test, the input varies for each recording. However, it is legitimate to assume that the input in the frequency domain is a constant in a narrow bandwidth. Thus, a bootstrap method can be applied to the normalized mode-isolated responses in the frequency domain for each sensor node. Figure 4-4 shows the flowchart of the procedures using the bootstrap method to propagate the measurement noises to the modal data, when $\mathrm{n}$ acceleration sensors are used and m dynamic response records are collected. As shown in Figure 4-4, the propagation process involves the extraction process; therefore, the uncertainties in the modal data are brought in by the measurement error and the modeling error associated with the extraction process as well.

\subsubsection{Modeling Errors Due to TDD}

Modeling error reflects the error in the output of a model due to the model inaccuracy. The sources of the error can come from the abstractions, assumptions, and approximations used in the modeling process. Conventionally, there are two types of modeling errors: parameter error and model error. Parameter error refers to the uncertainties in the model parameters in a given model due to the lack of knowledge of the parameter values. For example, the uncertainties in the stiffness and mass values in a finite element model (FEM) are parameter errors. Model error is associated with the model itself, reflecting the fact that there is no perfect numerical model to represent a real-world system and/or from the missing of a portion that is not included in the model. 
Reinert and Apostolakis (2006) gave a review of approaches to handle modeling errors, including model set expansion and prediction expansion methods. Model set expansion tries to use the advantages from different models and combines different models to produce a meta-model for a real system. Model prediction expansion, on the other hand, applies an "adjustment" directly to the prediction outcome from one model, with a single model being chosen as the best one. Therefore model set expansion can be treated as a special case of model set expansion by considering only one model. In this study, since only one specific extraction method is adopted to extract the modal data, using model prediction expansion is appropriate to estimate the modeling error associated with the extraction process.

Using additive model prediction expansion, the relation between the real modal data in the $i$ th mode (modal frequencies, $f_{i}$, and mode shape, $\boldsymbol{\varphi}_{i}$ ) and the extracted modal data $\left(\hat{f}_{i}\right.$ and $\hat{\boldsymbol{\varphi}}_{i}$ ) can be formulated as following,

$$
\begin{gathered}
f_{i}=\hat{f}_{i}+e_{f_{i}} \\
\boldsymbol{\varphi}_{i}=\hat{\boldsymbol{\varphi}}_{i}+\mathbf{e}_{\boldsymbol{\varphi}_{i}}
\end{gathered}
$$

where $e_{f_{i}}$ and $\mathbf{e}_{\boldsymbol{\varphi}_{i}}$ are the modeling errors in modal frequencies and mode shape due to the TDD process. They can be considered the "adjustments" to the prediction.

To assess the "adjustments", $e_{f_{i}}$ and $\mathbf{e}_{\varphi_{i}}$, one can directly compare the extracted modal data from TDD, $\hat{f}_{i}$ and $\hat{\boldsymbol{\varphi}}_{i}$, and the real values, $f_{i}$ and $\boldsymbol{\varphi}_{i}$. In system identification or a vibration-based NDT, a FEM usually needs to be constructed and validated by calibrating the unknown structural parameters (such as stiffness and/or 
mass) in a preliminary FEM using the extracted modal data. Given a certain setting in the vibration test, the uncertainties in the unknown structural prosperities can be used to estimate $e_{f_{i}}$ and $\mathbf{e}_{\boldsymbol{\varphi}_{i}}$. To obtain $\hat{f}_{i}$ and $\hat{\boldsymbol{\varphi}}_{i}$, the vibration responses used in the TDD are obtained by exciting a group of FEMs which is similar with the preliminary FEM but with different combinations of structural parameter values that are randomly drawn from the parameter ranges. Meanwhile, modal analysis is conducted for each FEM in that group to find the corresponding $f_{i}$ and $\boldsymbol{\varphi}_{i}$. When the error in the constructing FEM can be ignored, the difference between the identified modal data $\left(\hat{f}_{i}\right.$ and $\left.\hat{\boldsymbol{\varphi}}_{i}\right)$ and the real modal data $\left(f_{i}\right.$ and $\left.\boldsymbol{\varphi}_{i}\right)$ reflect the errors resulted from TDD. Note that if the modeling error in the constructing FEM needs to be considered, one can follow the methodology and specific implementations proposed by Haukaas and Gardoni (2009).

Using the difference between $f_{i}$ and $\hat{f}_{i}$, to estimate $e_{f_{i}}$ is straightforward. Given certain influencing factors, the mean, $\mu_{e f_{i}}$, and standard deviation, $\sigma_{e f_{i}}$, of $e_{f_{i}}$ can be estimated from the differences, $f_{i}-\hat{f}_{i}$. The differences between $\boldsymbol{\varphi}_{i}$ and $\hat{\boldsymbol{\varphi}}_{i}$ for the $i$ th mode can be considered a linear combination of mode shapes of other modes. This is because in the frequency domain the modes are overlapped due to the existence of the damping, which makes it impossible to obtain a pure mode-isolated response in the TDD method. Especially, a mode shape is influenced most by the nearest mode shape. Therefore, a linear regression model can be used to access mode shape error $\mathbf{e}_{\boldsymbol{\varphi}_{i}}$ for the $i$ th mode, which is written as follows, 


$$
\mathbf{e}_{\boldsymbol{\varphi}_{i}}=\sum_{j=1}^{N_{e}}\left[\alpha_{j, i}+\beta_{j, i}\left(f_{j}-f_{i}\right)^{\theta_{j, i}}\right] \boldsymbol{\varphi}_{j}\left(1-\delta_{i j}\right)+\sigma_{\boldsymbol{\varphi}_{i}} \boldsymbol{\varepsilon}
$$

where $N_{e}=$ number of modes used, $\alpha_{j, i}, \beta_{j, i}$, and $\theta_{j, i}$ are the model parameters for the $i$ th mode, $\delta_{i j}=$ Kronecker delta (i.e. $\delta_{i j}=1$, for $i=j ; \delta_{i j}=0$, for $i \neq j$ ), $\sigma_{\varphi_{i}}=$ standard deviation of the model error, and $\boldsymbol{\varepsilon}=$ a vector of normal random variables with zero mean and unit variance. The model parameters are estimated by the Bayesian updating rule (Gardoni et al. 2003). One could use locally uniform distributions for the model parameters as the non-informative priors in the Bayesian updating rule. Note that the further a mode is from the $i$ th mode, the less contribution that mode has to the modeling error $\mathbf{e}_{\boldsymbol{\varphi}_{i}}$. Moreover, the closeness of two modes can be measured by the difference between the corresponding modal frequencies, which is the reason that the term $\beta_{j, i}\left(f_{j}-f_{i}\right)^{\theta_{j, i}}$ is included in Eq. (7). A stepwise deletion procedure (Gardoni et al. 2002) is adopted to eliminate unimportant terms in Eq. (7) to obtain a parsimonious model without losing accuracy. Overall, the steps of accessing $e_{f_{i}}$ and $\mathbf{e}_{\boldsymbol{\varphi}_{i}}$ are shown in Figure 4-5. Although the procedure described here is in terms of a particular modal parameter identification scheme, TDD, it can be extended to any other identification process.

\subsubsection{Sample size}

In the flowchart shown in Figure 4-4, the sample size for the bootstrap needs to be determined. Generally, one thousand samples are often enough for a first look. 
However, given available computing power and time, it is reasonable to use as many samples as possible.

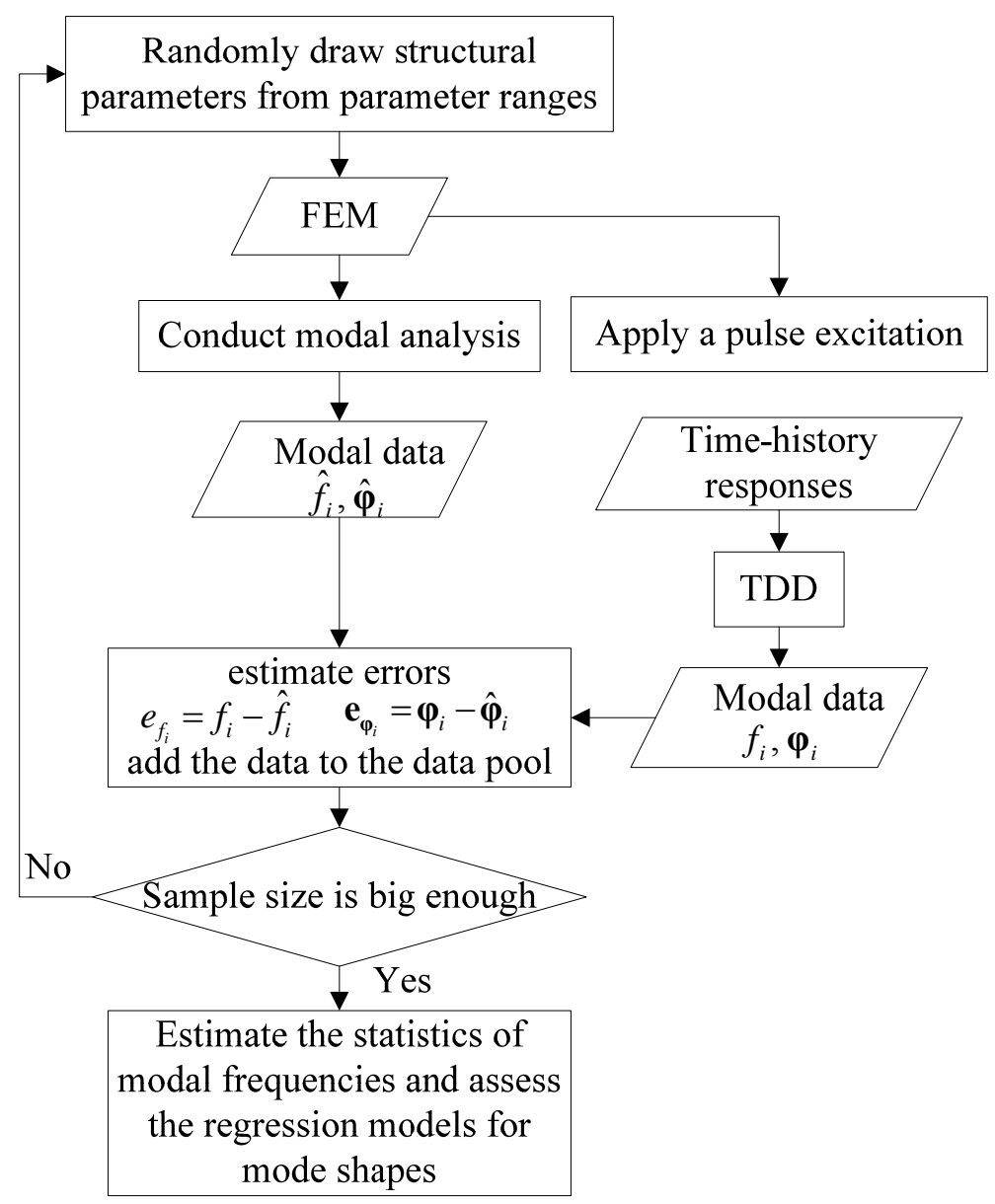

Figure 4-5. Flowchart of the procedures to estimate the modeling error in TDD

The sample size in the flowchart in Figure 4-5 is determined by the maximum of two sample sizes, where one sample size, $n_{f}$, is for determining the statistical interference of $e_{f_{i}}$, and the other sample size, $n_{\varphi}$, is for assessing the regression models for $\mathbf{e}_{\varphi_{i}}$. 
The sample size, $n_{f}$ can be estimated from confidence intervals. With $100(1-\alpha) \%$ confidence where $\alpha$ refers to the significance level, the following relationships hold,

$$
\begin{gathered}
\hat{\mu}_{e f}-Z_{\alpha / 2} \frac{S_{e f}}{\sqrt{n_{\mu f}}} \leq \mu_{e f} \leq \hat{\mu}_{e f}+Z_{\alpha / 2} \frac{S_{e f}}{\sqrt{n_{\mu f}}} \\
\frac{n_{\sigma f}}{\chi_{\alpha / 2}^{2}} S_{e f}{ }^{2} \leq \sigma_{e f}{ }^{2} \leq \frac{n_{\sigma f}}{\chi^{2}{ }_{1-\alpha / 2}} S_{e f}{ }^{2}
\end{gathered}
$$

where $\mu_{e f}=$ mean of $e_{f_{i}}, \hat{\mu}_{e f}=$ population mean, $S_{e f}=$ population of standard deviation, $\sigma_{e f}=$ standard deviation of $e_{f_{i}}, Z_{\alpha / 2}=$ critical value at the vertical boundary for the area of $\alpha / 2$ in the right tail of the standard normal distribution, and $\chi_{\alpha / 2}^{2}=$ critical value at the vertical boundary for the area of $\alpha / 2$ in the right tail of the $\chi^{2}$ distribution. Given certain confidence intervals for $\mu_{e f}$ and $\sigma_{e f}$, then the sample size $n_{f}$ can be calculated by $n_{f}=\max \left(n_{\mu f}, n_{\sigma f}\right)$.

As shown in Eq. (4.23), a linear regression model can be used to access mode shape error $\mathbf{e}_{\boldsymbol{\varphi}_{i}}$. The sample size, $n_{\varphi}$, needs to be determined for the multivariate regression model, Eq. (4.23), to ensure sufficient statistical power to detect a significant effect. Followed the theory in Cohen (1988), four input parameters are needed to determine $n_{\varphi}:$ (1) a significance confidence level $\alpha,(2)$ a target power level, $\gamma$, (3) the effect size, ES, and (4) the number of predictors used, $p$. For a regression model, the power level of the F-test refers to the probability that one rejects the null hypothesis while the alternative hypothesis is true. To calculate the power, the F-distribution and 
the noncentral F-distribution probability density function are used and they are respectively written as

$$
\begin{gathered}
p(x)=\frac{1}{B\left(\frac{v}{2}, \frac{u}{2}\right)}\left(\frac{u x}{u x+v}\right)^{\frac{u}{2}}\left(1-\frac{u x}{u x+v}\right)^{\frac{v}{2}} x^{-1} \\
p(g)=\sum_{k=0}^{\infty} \frac{e^{-\lambda / 2}(\lambda / 2)^{k}}{B\left(\frac{v}{2}, \frac{u}{2}+k\right) k !}\left(\frac{u}{v}\right)^{\frac{u}{2}+k}\left(\frac{v}{u+v g}\right)^{\frac{v+u}{2}+k} g^{\frac{v}{2}-1+k}
\end{gathered}
$$

where $B(\cdot)=$ Beta function, $u=$ number of predictors, $p$, or numerator degrees of freedom, $\quad v=$ denominator degrees of freedom, $\lambda=$ noncentrality parameter, and $g=$ critical value of $F$-distribution. Note that no intercept is included in Eq. (4.23), thus the sample size $\hat{n}=u+v$; otherwise, if intercept is included in a regression model, $\hat{n}=u+v+1$. The effect size, ES, describes the degree to which the null hypothesis is false. Therefore, the larger the effect size is, the greater the degree to which the alternative hypothesis is manifested. Conventionally, ES of $0.02,0.15$, and 0.35 are considered as small, medium, and large, respectively. With the four input values, the sample size, $n_{M S}$, can be calculated by using the following steps:

i) Set a desired power level $\gamma$, an effect size ES, and a significance confidence level $\alpha$, and set initial value $v=p$.

ii) Calculate critical value of F-distribution $g$ through Eq. (4.26), with given values of $\alpha, u(=p)$, and $v$.

iii) Compute $\lambda=E S \cdot(u+v)$. 
iv) With the values of $g, u(=p), v$, and $\lambda$, estimate the value of the noncentral Fdistribution through Eq. (4.27).

v) Compute the power by calculating the cumulative area under the standard normal curve from zero to the value of the noncentral F-value estimated from the previous step.

vi) If the power computed from step $\mathrm{v}$ ) is less the desired power level $\gamma$, increase $v$ value and repeat step ii) through step v).

vii) Determine the sample size $n_{\varphi}=u+v$.

\subsection{Illustration}

In this study, the investigation of the effects of the measurement, modeling and statistical errors is illustrated with a numerical example. A FEM of a two-span continuous aluminum beam on elastic supports shown in Figure 4-6 is selected as the target structure, which has been previously analyzed by Stubbs and Kim (1996). The FEM is built in OpenSees (McKenna and Fenves 2002) using 50 elastic beam-column elements with elastic springs for the supports, where the mass density of the beam $\rho=2710 \mathrm{~kg} / \mathrm{m}^{3}$, the cross section area $A=1.05 \times 10^{-3} \mathrm{~m}^{2}$, the second moment of area around the vertical z-axis $I_{z}=9.57 \times 10^{-7} \mathrm{~m}^{4}$, the second moment of the area around the transverse y-axis $I_{y}=7.23 \times 10^{-7} \mathrm{~m}^{4}$, the Poisson's ratio $v=0.33$, the elastic modulus of the beam $E_{b}=56 \mathrm{GPa}$, and the stiffness of the support springs $K_{1}=K_{2}=6.0 \times 10^{-5} \mathrm{~N} / \mathrm{m}$ and $K_{3}=1.2 \times 10^{-4} \mathrm{~N} / \mathrm{m}$. 


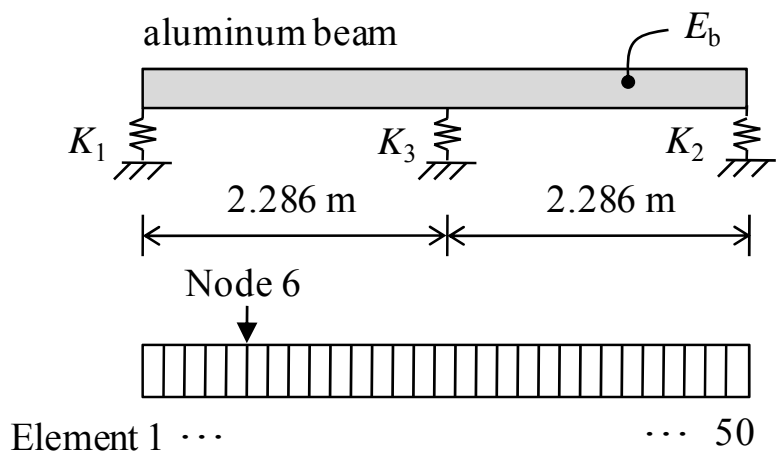

Figure 4-6. Schematic of the example beam and the FEM

A vibration test is conducted by applying a pulse force at node 6 of the beam. The effect of different error levels $e_{1}$ in the sensors is simulated by adding to the time history records a Gaussian white noise with standard deviations equal to $0.5 \%, 1 \%, 2 \%$, and 3\%, of the amplitude of the acceleration responses. The recording time is $4 \mathrm{~s}$ with sampling time $0.0001 \mathrm{~s}$. The first three modal frequencies and mode shapes are used in the damage detection. 

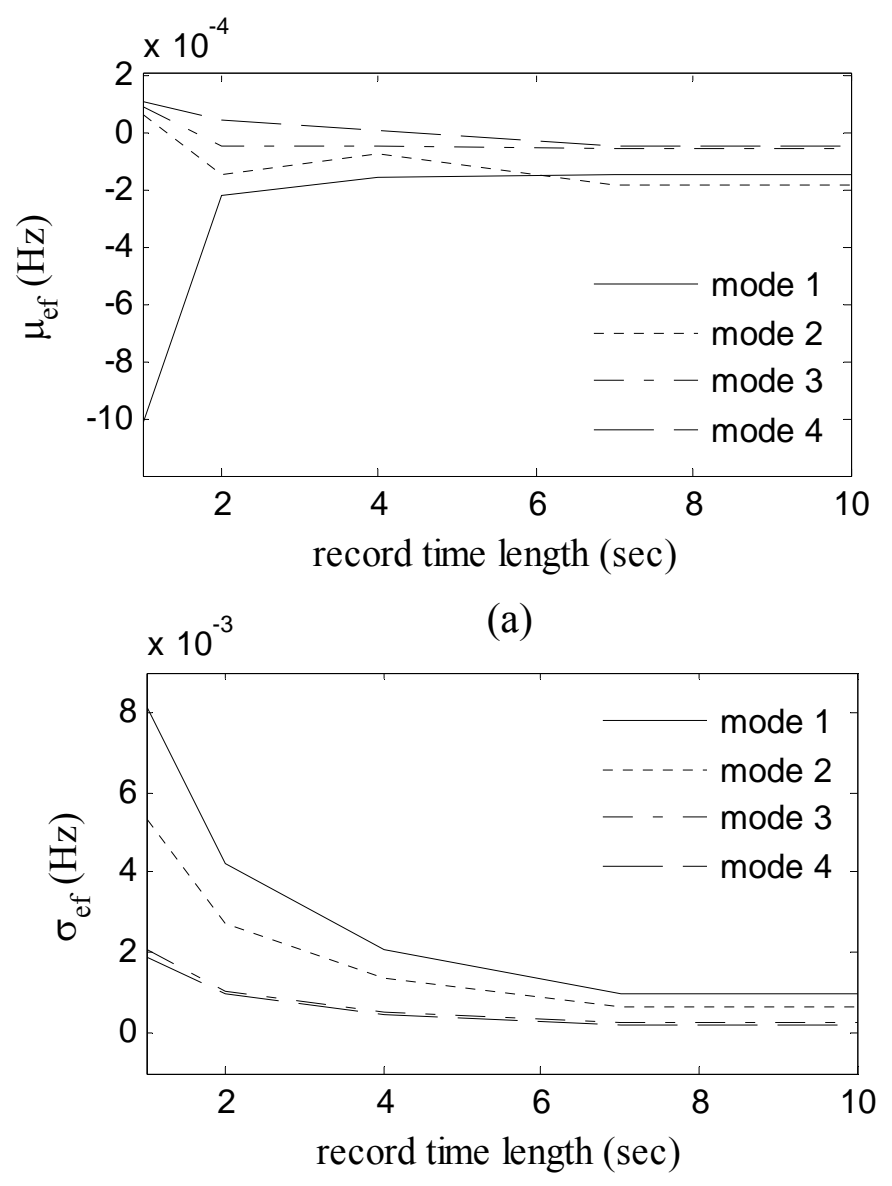

(b)

Figure 4-7. Mean (a) and standard deviation (b) of modeling error in modal frequencies

To estimate the effects of modeling error in the modal parameters due to TDD following the flowchart shown in Figure 4-5, one needs to determine the sample size first. If a significance confidence level $5 \%$ is chosen, and confidence intervals for $\mu_{e f}$ and $\sigma_{e f}$ are set to be $\left[\mu_{e f}-S / 5, \mu_{e f}+S / 5\right]$ and $\left[86.4 \% \cdot S^{2}, 116.4 \% \cdot S^{2}\right]$ respectively, then $n_{\mu f} \approx 100$ and $n_{\sigma f} \approx 350$. Followed the steps for determining $n_{\varphi}$, with selected 
$\alpha=0.05, \gamma=0.9, E S=0.15$, and $p=6$, the $n_{\varphi}=123$. Thus the sample size should be at least 350. A total of $400 \mathrm{FEMs}$ is generated with the same configurations as the preliminary FEM by varying the stiffness properties of the structures, $E_{b}, K_{1}\left(=K_{2}\right)$, and $K_{3}$. Note that the modeling error due to TDD is a function of the corresponding influence factors described previously. Figure 4-7 shows how the modeling error due to TDD in the first four frequencies varies when one of the influence factors, the response length, increases. Notice that after exceeding a certain response length, the error does not change much anymore. Other influence factors can be studied in a similar manner.

The regression models of the mode shape error $\mathbf{e}_{\varphi_{i}}$ of the first three modes are obtained following the formulation given by Eq. (4.23)

$$
\begin{gathered}
\mathbf{e}_{\boldsymbol{\varphi}_{1}}=\alpha_{1,1} \boldsymbol{\varphi}_{2}+\alpha_{2,1} \boldsymbol{\varphi}_{3}+\alpha_{3,1} \boldsymbol{\varphi}_{4}+\beta_{1,1}\left(f_{2}-f_{1}\right)^{\theta_{1,1}} \boldsymbol{\varphi}_{2}+\left(\sigma_{0,1} \mathbf{I}+\sigma_{1,1}\left|\boldsymbol{\varphi}_{2}\right|\right) \varepsilon+\sigma_{1} \boldsymbol{\varepsilon} \\
\mathbf{e}_{\boldsymbol{\varphi}_{2}}=\alpha_{1,2} \boldsymbol{\varphi}_{1}+\alpha_{2,2} \boldsymbol{\varphi}_{3}+\alpha_{3,2} \boldsymbol{\varphi}_{4}+\beta_{1,2}\left(f_{2}-f_{1}\right)^{\theta_{1,2}} \boldsymbol{\varphi}_{1}+\left(\sigma_{0,2} \mathbf{I}+\sigma_{1,2}\left|\boldsymbol{\varphi}_{1}\right|\right) \varepsilon+\sigma_{2} \boldsymbol{\varepsilon} \\
\mathbf{e}_{\boldsymbol{\varphi}_{3}}=\alpha_{1,3} \boldsymbol{\varphi}_{1}+\alpha_{2,3} \boldsymbol{\varphi}_{3}+\alpha_{3,3} \boldsymbol{\varphi}_{4}+\beta_{1,3}\left(f_{4}-f_{3}\right)^{\theta_{1,3}} \boldsymbol{\varphi}_{4}+\left(\sigma_{0,3} \mathbf{I}+\sigma_{1,3}\left|\boldsymbol{\varphi}_{4}\right|\right) \varepsilon+\sigma_{2} \boldsymbol{\varepsilon}
\end{gathered}
$$

where the statistics of the model parameters $\alpha_{j, i}, \beta_{j, i}, \theta_{j, i}$, and $\sigma_{j, i}$ are shown in Table 4-2 and they are estimated using the Bayesian approach (Box and Tiao 1992).

The preliminary FEM is the same as the one used for the target baseline where $E_{b}=80 \mathrm{GPa}, K_{1}=K_{2}=3.0 \times 10^{-5} \mathrm{~N} / \mathrm{m}$ and $K_{3}=0.6 \times 10^{-4} \mathrm{~N} / \mathrm{m}$. In this example, since the preliminary FEM is a FEM constructed in the same manner as the target structure, $e_{3 b}$ is zero. Details on the quantification of $e_{3 b}$ in other cases can be found in (Haukaas and Gardoni 2009). Table 4-3 compares the modal frequencies for the preliminary, 
target, and identified baseline FEMs. The differences of modal frequencies between the target structure and preliminary FEMs indicate that the error $e_{3 a}$ exists in the preliminary FEM. This error can be reduced by calibrating $E_{b}, K_{1}\left(=K_{2}\right)$, and $K_{3}$ in the model updating process.

Table 4-2. Statistics of model parameters

\begin{tabular}{|c|c|c|c|c|c|c|c|c|c|}
\hline & & $\alpha_{1}$ & $\alpha_{2}$ & $\alpha_{3}$ & $\beta_{1}$ & $\theta_{1}$ & $\sigma_{0}$ & $\sigma_{1}$ & $\sigma$ \\
\hline \multirow{2}{*}{ mode 1} & mean & $-1.41 \mathrm{E}-04$ & $-2.57 \mathrm{E}-05$ & $-1.96 \mathrm{E}-05$ & $-2.03 E+00$ & $1.41 \mathrm{E}-01$ & $5.38 \mathrm{E}$ & $.68 \mathrm{E}-04$ & $51 \mathrm{E}-07$ \\
\hline & $\operatorname{cov}^{\dagger}$ & $6.65 \mathrm{E}-03$ & $1.09 \mathrm{E}-02$ & $1.38 \mathrm{E}-02$ & $1.06 \mathrm{E}-03$ & $3.42 \mathrm{E}-03$ & $5.53 \mathrm{E}$ & $.93 \mathrm{E}-03$ & - \\
\hline \multirow{2}{*}{ mode 2} & mean & $9.10 \mathrm{E}-05$ & $-4.10 \mathrm{E}-05$ & $-2.59 \mathrm{E}-05$ & $-1.70 \mathrm{E}+00$ & $1.51 \mathrm{E}-01$ & $1.23 \mathrm{E}$ & .08E-03 & $61 \mathrm{E}-06$ \\
\hline & $\operatorname{cov}^{\dagger}$ & $1.88 \mathrm{E}-02$ & $1.04 \mathrm{E}-02$ & 1.39E-02 & 7.75E-04 & 2.19E-03 & $9.69 \mathrm{E}-$ & $.87 \mathrm{E}-04$ & - \\
\hline \multirow{2}{*}{ mode 3} & mean & $1.68 \mathrm{E}-04$ & $2.20 \mathrm{E}-04$ & $-1.34 \mathrm{E}-03$ & $-3.86 \mathrm{E}+00$ & $-3.55 \mathrm{E}+01$ & $8.98 \mathrm{E}$ & $.70 \mathrm{E}-03$ & $10 \mathrm{E}-05$ \\
\hline & $\operatorname{cov}^{\dagger}$ & $1.91 \mathrm{E}-01$ & $1.45 \mathrm{E}-01$ & $2.66 \mathrm{E}-02$ & $6.61 \mathrm{E}-03$ & 4.07E-02 & $9.52 \mathrm{E}-$ & $.12 \mathrm{E}-03$ & - \\
\hline
\end{tabular}

$\dagger \operatorname{cov}=$ coefficient of variance $=$ standard deviation $/$ mean

In the Bayesian model updating, one can validate the normality assumption of $\xi_{j}$ and the sampling size in MCMC is sufficiently large so that the errors $e_{4 a}$ and $e_{4 b}$ can be ignored. In addition, $e_{1}$ is simulated as white noises that contain broadband information in the frequency domain, therefore, the $\xi_{j}$ is not influenced by $e_{1}$. As such, in this example $\xi_{j}$ results from $e_{2}$ and $e_{3 a}$. After applying the Bayesian model updating, $\hat{f}_{j}$ agree well with $f_{j}$ (as shown in Table 4-3), and the errors $e_{3 a}$ in the identified baseline has been significantly reduced (as shown in Table 4-4). These results indicate that the preliminary FEM has been updated successfully. As such, in this illustration, the DI is influenced by $e_{1}$, and $e_{2}$. 
Table 4-3. Comparison of modal frequencies $(\mathrm{Hz})$

\begin{tabular}{ccccc}
\hline \multirow{2}{*}{ Mode } & $\begin{array}{c}\text { Preliminary }^{\dagger} \\
\hat{f}_{j}\end{array}$ & Target $^{\ddagger}$ & \multicolumn{2}{c}{ Identified $\hat{f}_{j}$} \\
\cline { 4 - 5 } \cline { 3 - 5 } & 40.4487 & 36.6270 & 36.6042 & 0.0240 \\
2 & 58.8472 & 55.1379 & 55.1042 & 0.0376 \\
3 & 150.6397 & 141.6217 & 141.6214 & 0.1379 \\
\hline frequencies obtained from modal analysis & & \\
* frequencies obtained from TDD & & &
\end{tabular}

Table 4-4. Estimates of $e_{3 a}$ for the example beam

\begin{tabular}{|c|c|c|c|c|}
\hline \multirow{2}{*}{ Parameter } & \multirow{2}{*}{$\begin{array}{l}\text { Preliminary } \\
\text { FEM }\end{array}$} & \multicolumn{3}{|c|}{ Identified FEM } \\
\hline & & Mean & $\begin{array}{c}\text { Mean } \\
-1 \text { St. Dev }\end{array}$ & $\begin{array}{c}\text { Mean } \\
+1 \text { St. Dev }\end{array}$ \\
\hline$E_{b}$ & $25 \%$ & $0.16 \%$ & $0.11 \%$ & $0.21 \%$ \\
\hline$K_{1}\left(=K_{2}\right)$ & $50 \%$ & $-1.63 \%$ & $-3.15 \%$ & $-0.11 \%$ \\
\hline$K_{3}$ & $50 \%$ & $0.16 \%$ & $-0.15 \%$ & $0.47 \%$ \\
\hline
\end{tabular}

Four damage scenarios are introduced to the target structure: Damage Case 1: the flexural stiffness in beam Element 16 is reduced by $2 \%, 6 \%$, and 10\%; Damage Case 2: the flexural stiffness in beam Element 48 is reduced by $2 \%, 6 \%$, and $10 \%$; Damage Case 3: the flexural stiffness in beam Elements 16 and 30 is reduced 2\%, 6\%, and 10\%; and Damage Case 4: the flexural stiffness in beam Elements $11-15$ is reduced 2\%, $6 \%$, and $10 \%$. Different percentage deductions reflecting different level of damage are used to study influences of the severity. Damage Cases 1 and 2 are designed to evaluate the effects of the proximity of the support to the damaged area. Damage Case 3 is used to 
illustrate situations when more than one damage location is present. Damage Case 4 is used to study the effects of the size of the damage area.

It is found that the modal frequencies extracted from TDD for all the damage cases even with the most severe damage (10\% flexural stiffness reduction) are close to the ones for the case where no damage is introduced. Table 4-5 shows a comparison between the modal frequencies for the undamaged beam and the four damage cases with $10 \%$ flexural stiffness reduction. This observation further confirms that the modal frequencies of a structure are not sensitive to the damages at local level (Wang and Haldar 1994). Thus, it is legitimate to believe that when the modal frequencies of a damaged structure are used to update the preliminary FEM, as proposed in this study, the updated FEM can be considered as an accurate baseline for the damaged structure.

Table 4-5. Comparison of modal frequencies of no damage case and damage cases ${ }^{\dagger}$

$(\mathrm{Hz})$

\begin{tabular}{cccccc}
\hline Mode & $\begin{array}{c}\text { No } \\
\text { damage }\end{array}$ & $\begin{array}{c}\text { Damage } \\
\text { Case 1 }\end{array}$ & $\begin{array}{c}\text { Damage } \\
\text { Case 2 }\end{array}$ & $\begin{array}{c}\text { Damage } \\
\text { Case 3 }\end{array}$ & $\begin{array}{c}\text { Damage } \\
\text { Case 4 }\end{array}$ \\
\hline 1 & 36.6270 & 36.5588 & 36.6192 & 36.5359 & 36.2470 \\
2 & 55.1379 & 55.1103 & 55.1223 & 55.0909 & 54.7927 \\
3 & 141.6217 & 141.4680 & 141.5126 & 141.2381 & 141.4453 \\
\hline${ }^{\dagger}$ considering 10\% flexural stiffness reduction & &
\end{tabular}

One objective of this illustration is to study how the errors in the damage detection process influence the damage indexes. Figures 4-8 to 4-10 show the variability of $Z$ when different levels of measurement noise (error $e_{1}$ ) are considered for Damage Case 1. Figure 4-11 shows the variability of $Z$ for each beam element when only the 
error $e_{2}$ is considered. It is observed that when the damage severity increases, the effect from $e_{2}$ on $Z$ decreases. By comparing Figures $4-8$ to $4-10$ with Figure 4-11, it is found that the variability in $Z$ due to $e_{1}$ is larger than the one due to $e_{2}$, indicating that the measurement error has more effects on the damage detection results than the modeling error in TDD. The same observations can be made for the other three damage cases and for brevity are not shown in the dissertation.

Given the same level of $e_{1}$, Figures 4-8 to 4-10 show the influence of the damage severity level on $Z$. For a low level of damage (2\% flexural stiffness reduction), the $Z$ values of damaged elements can hardly be differentiated from other elements regardless of the threshold used even for the smallest $e_{1}$. However, when the severity becomes larger, this differentiation becomes more obvious. This concludes that if the damage severity is too small, it is difficult to detect damage even when the measurement noise level is small. Additionally, it is noticed from Figures 4-8 to 4-10 that the mean values of $Z$ decreases and the standard deviations of $Z$ increases over the damaged element with increasing $e_{1}$. As anticipated, a high level of $e_{1}$ obscures the signal that can be used to detect damages and brings in more uncertainties. Furthermore, the elements next to the support (elements 1 and 50), which do not have stiffness reduction, also have high values of $Z$, which might lead to false detections. These observations can also be made for the other three damage cases. 


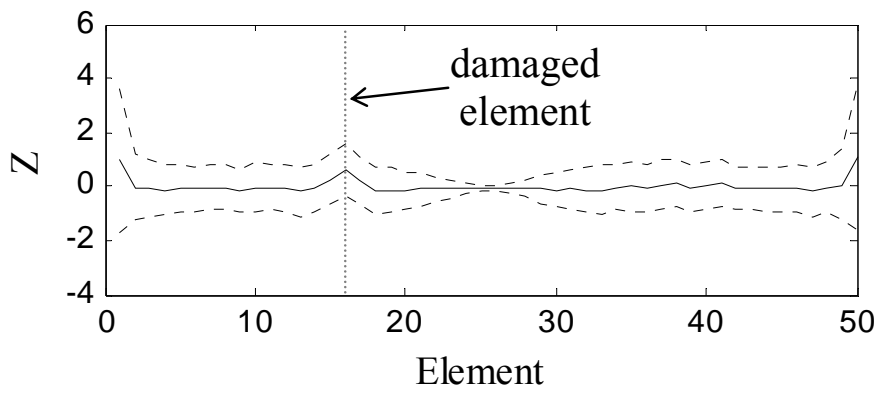

(a) $2 \%$ stiffness reduction

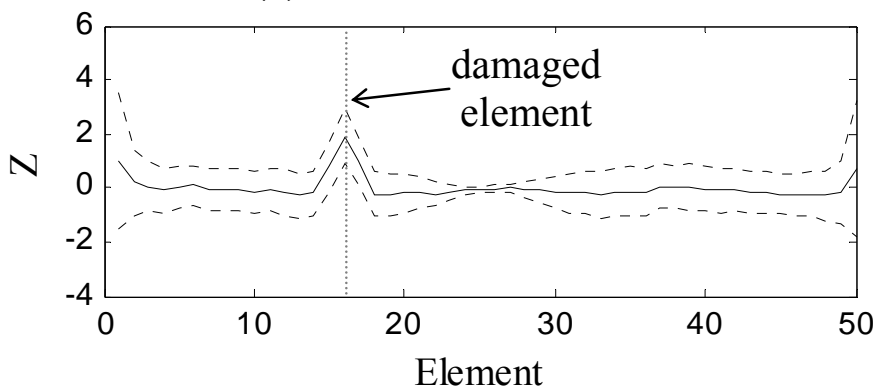

(b) $6 \%$ stiffness reduction

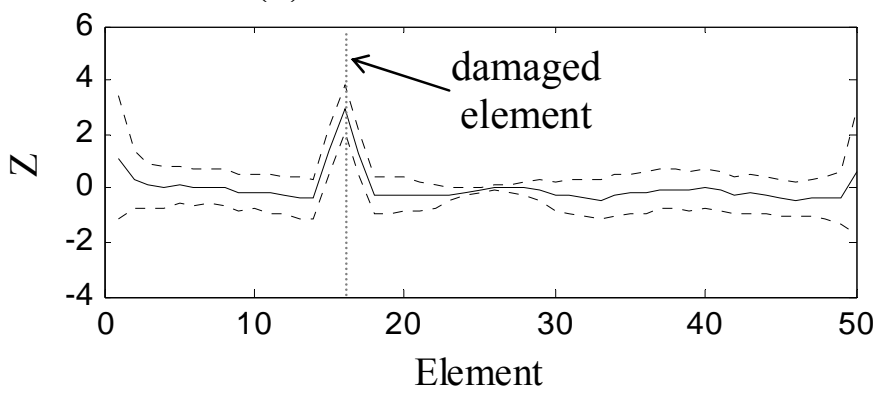

(c) $10 \%$ stiffness reduction

Figure 4-8. $Z$ values (solid line: mean, dotted line: mean \pm 1 standard deviation) for the beam elements with Damage Case 1 under $1 \%$ noise level 


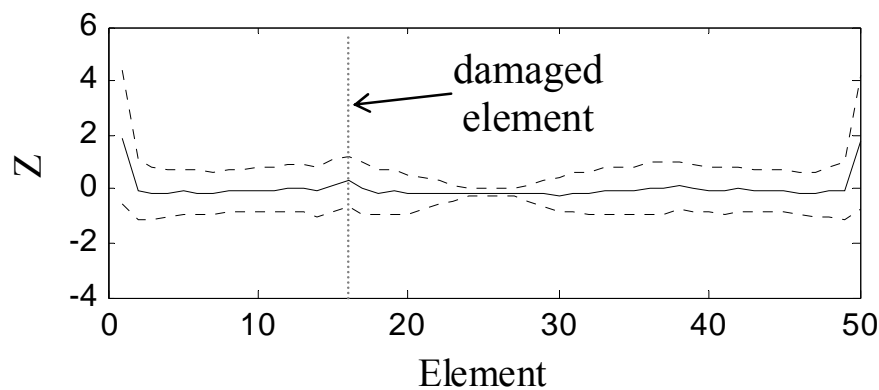

(a) $2 \%$ stiffness reduction

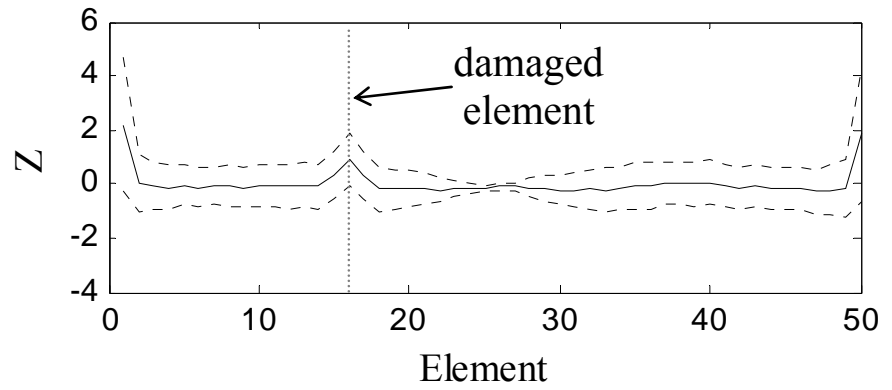

(b) $6 \%$ stiffness reduction

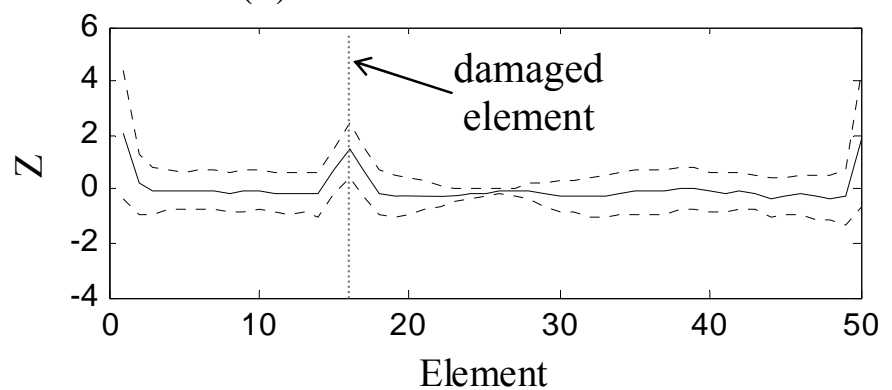

(c) $10 \%$ stiffness reduction

Figure 4-9. $Z$ values (solid line: mean, dotted line: mean \pm 1 standard deviation) for the beam elements with Damage Case 1 under 2\% noise level 


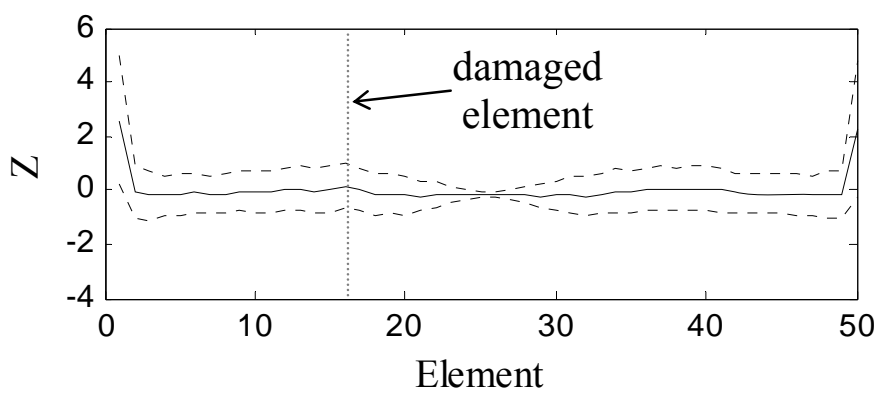

(a) $2 \%$ stiffness reduction

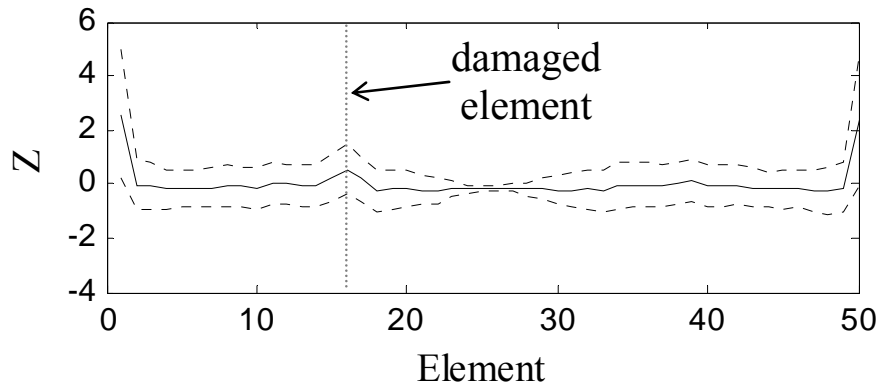

(b) $6 \%$ stiffness reduction

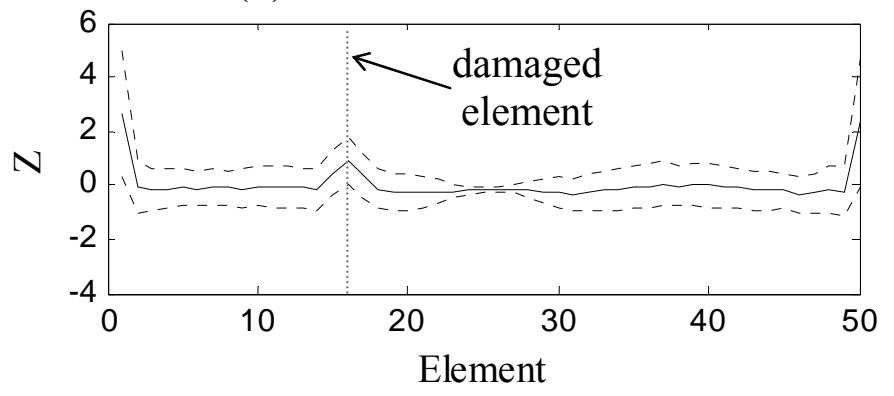

(c) $10 \%$ stiffness reduction

Figure 4-10. $Z$ values (solid line: mean, dotted line: mean \pm 1 1standard deviation) for the beam elements with Damage Case 1 under $3 \%$ noise level 


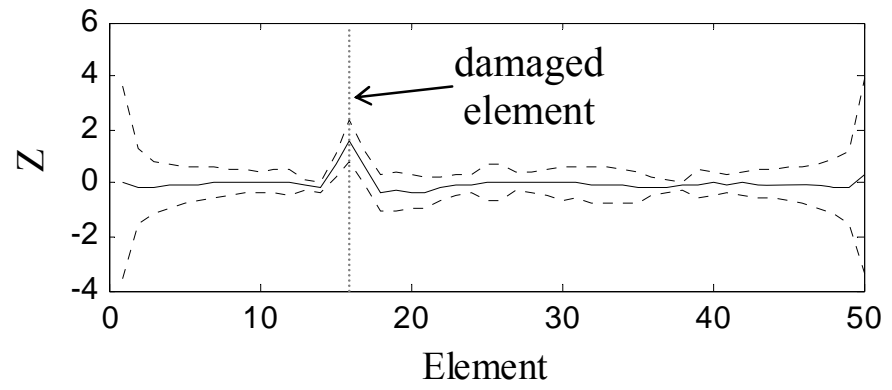

(a) $2 \%$ stiffness reduction

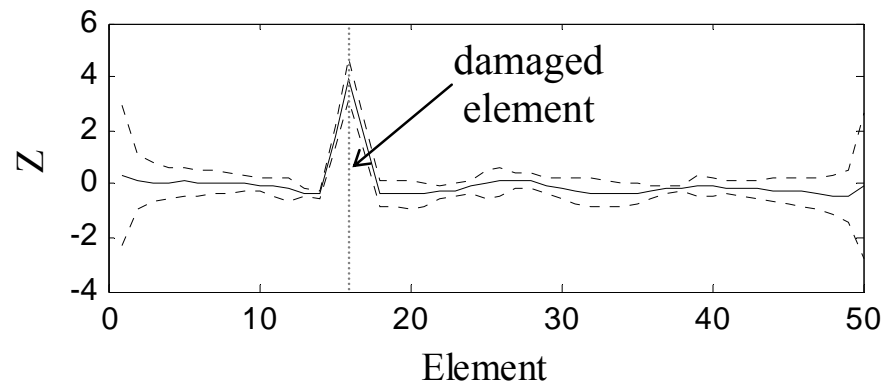

(b) $6 \%$ stiffness reduction

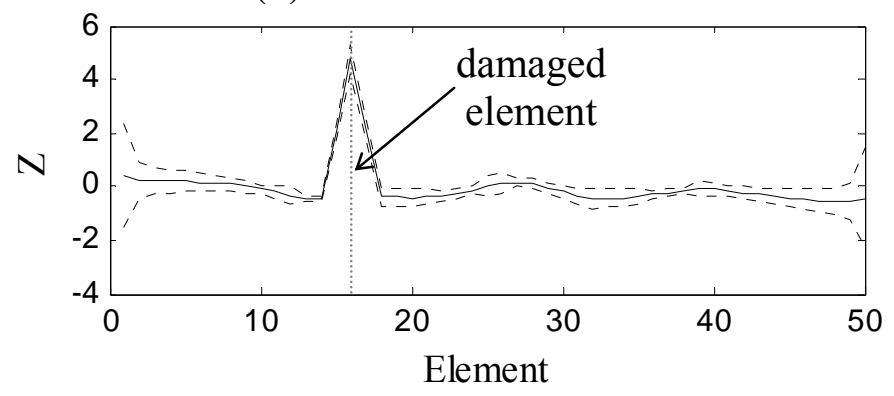

(c) $10 \%$ stiffness reduction

Figure 4-11. $Z$ values (solid line: mean, dotted line: mean \pm 1 standard deviation) for the beam elements with Damage Case 1 considering error $e$, 


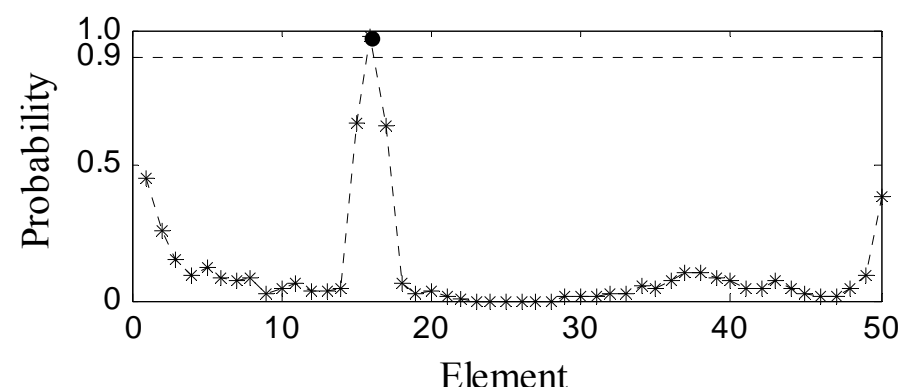

(a) Damage Case 1

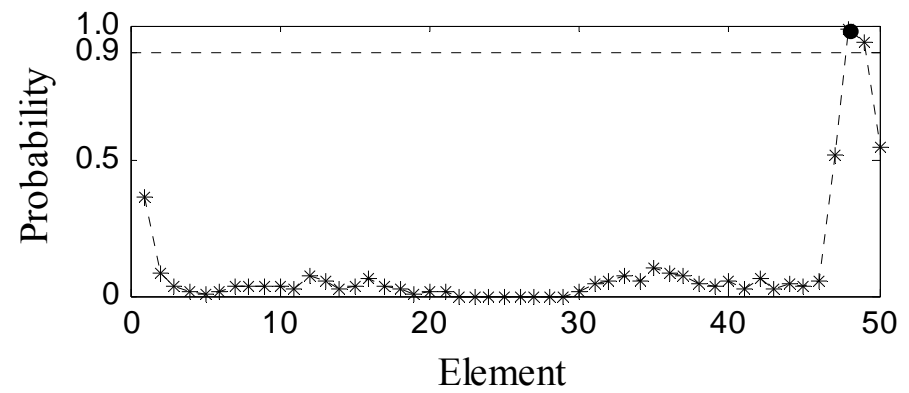

(b) Damage Case 2

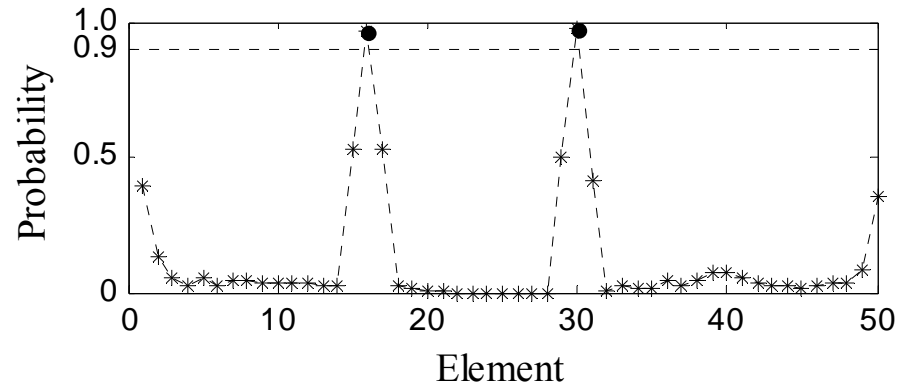

(c) Damage Case 3

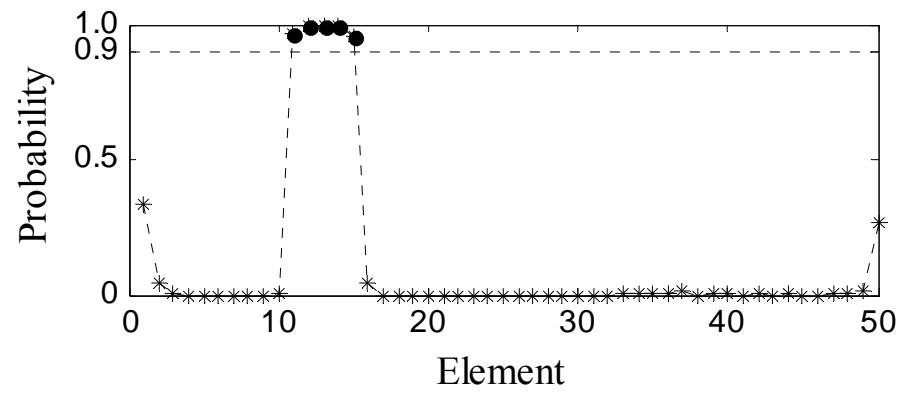

(d) Damage Case 4

Figure 4-12. Probability of damage detection for Damage Cases 1-4 with $10 \%$ flexural stiffness reduction under $1 \%$ noise level using $\lambda=2.0$ 
For all the damage cases, if a threshold is chosen appropriately, the damage location can be identified. Figure 4-12 shows the probability of detection for the all four damage cases with $10 \%$ flexural stiffness reduction under $1 \%$ noise level using a selected threshold $\lambda_{i}=1.0$, which is a typical threshold value used in the literature (Alvandi and Cremona 2006; Park et al. 2001). The star points (•) in Figure 4-12 indicate the probability of correct detection $P(I D \mid D)$, while the dots $\left(^{*}\right)$ indicate the probability of false detection $P(I D \mid N D)$ For these four damage cases, $P(I D \mid D)>0.9$, which shows that the damage detection approach can successfully detection damage location for the four damage scenarios considered. However, the elements adjacent to the damaged elements in the first three damage cases have a high probability of false detection $P(I D \mid N D)$, which indicates a difficulty in assessing the size of the damage area. Furthermore, as pointed out previously, the undamaged elements 1 and 50 also show relatively high values of $P(I D \mid N D)$, which is in part due to the use of the spline interpolation.

The natural question followed by the observations in Figure 4-12 is how to choose an appropriate threshold for the DIM to reach a compromise between maximizing $P(I D \mid D)$ and minimizing $P(I D \mid N D)$. Figure 4-13 shows the probability of damage detection for Damage Case 1 with 10\% flexural stiffness reduction and $1 \%$ measurement noise when three different thresholds are chosen. The selection of $\lambda_{i}=1.0$ and $\lambda_{i}=3.0$ is used to assess the effects of a higher thresholds on $P(I D \mid D)$ and $P(I D \mid N D)$, and the selction of $\lambda_{i}=1.0+\sigma_{i}$, where $\sigma_{i}=$ the standard deviation of $Z_{i}$ 
for $i$ th element is used to assess the effects of a varying the threshold over the length of the beam. The threshold $\lambda_{i}=1.0+\sigma_{i}$ sets high criteria for the non-damaged elements next to the supports, where $\sigma_{i}$ is larger. As discussed before, when the threshold is low (such as $\left.\lambda_{i}=1.0\right), P(I D \mid N D)$ is too high for non-damaged elements such as Elements 1 and 50. By increasing the threshold to $\lambda_{i}=3.0$, although it helps decrease $P(I D \mid N D)$, it also significantly reduce $P(I D \mid D)$. As shown in Figure 4-13, the proposed threshold $\lambda_{i}=1.0+\sigma_{i} \quad$ greatly improves the results maximizing $P(I D \mid D)$ and minimizing $P(I D \mid N D)$. Based on observation that the mean values of $Z_{i}$ decreases and the standard deviations of $Z_{i}$ increases over the damaged element with increasing $e_{1}$, the threshold should also vary with different levels of measurement noises.

\subsection{Conclusions}

This section presents a probabilistic damage detection approach that is based on modal parameters extracted from ambient vibration responses. The proposed approach uses a Bayesian model updating to develop an accurate baseline using the extracted modal frequencies, and the damage index method (DIM) to accurately detect the local damage using the extracted mode shapes. The uncertainties in the proposed approach and how they affect the damage detection are discussed. Considering these uncertainties, the proposed approach is illustrated using a numerical example.

It is found that the uncertainties in the damage detection have considerably effect on the damage detection results, indicating that to present the detection results in a 
probabilistic form is necessary. In particular, the measurement error due to the noise in the ambient vibration has the largest effect on the damage detection. Therefore, it is suggested to allot more effort to improve measurement devices and/or data processing. With low damage level such as stiffness deduction less than $2 \%$, it may impossible to detect the damage using the DIM even under low level of measurement error. Additionally, it is observed that the non-damaged elements next to the supports show high probability of false detection for all the damage cases considered in the numerical example. This is partially due to the use of spline interpolation to obtain the mode shape curvature at the boundaries in DIM. To choose an appropriate threshold in DIM to identify the local damage locations, it is found that the proposed threshold is most effective to minimize the false detection probability and maximize the correct detection probability. For different measurement noise level, the threshold level should to be defined differently.

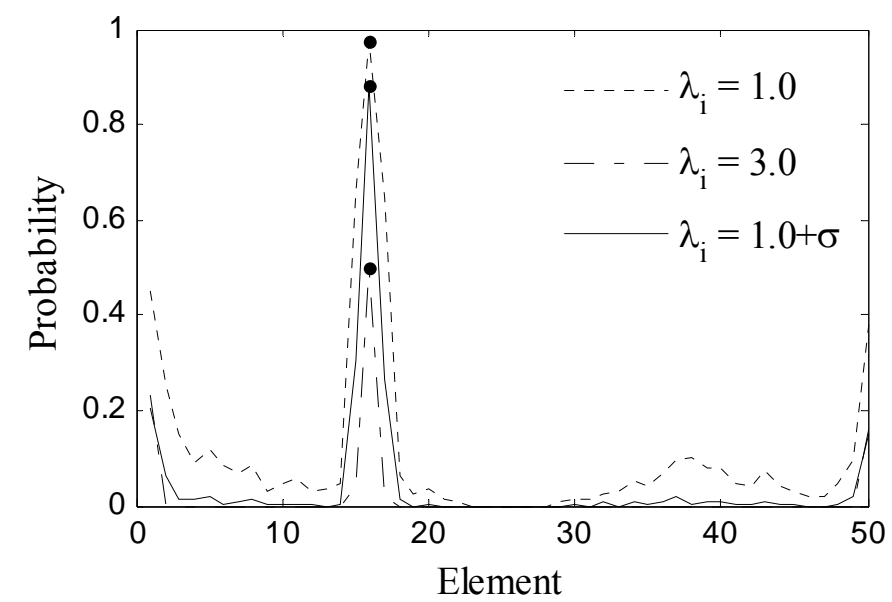

Figure 4-13. Probability of damage detection for Damage Case 1 with $10 \%$ flexural stiffness reduction under $1 \%$ noise level 
Since the modal parameters are usually obtained though a vibration test, the proposed approach cannot keep track of the instantaneous stiffness change, such as during an earthquake event. However, it can be used to evaluate the structural performance before and after an event. The proposed method is able to evaluate the reliability of the damage estimation results probabilistically and quantitatively, requiring only the dynamic responses. Thus, this proposed framework can be used to evaluate the current and future performance of a structure. Furthermore, the structural parameters used in the performance evaluation can be continuously updated when new modal parameters become available. 


\section{LOCAL NONDESTRUCTIVE TESTING}

\subsection{Introduction}

As a general index of concrete strength, compressive strength of concrete, $f_{c}$, is important in the performance assessment of existing RC structures. The concrete compressive strength, $f_{c}$, is considered as a general index of concrete strength and can be determined by NDT methods. ACI Committee 228 (2003) provides a guideline for using NDT methods to predict $f_{c}$. The various NDT methods include rebound hammer test, ultrasonic pulse velocity (UPV) test, maturity, and cast-in-place cylinders. As found by many researchers (Samarin and Meynink 1981; Miretti et al. 2004; Hola and Schabowicz 2005), the combined methods that refer to the use of two or more in-situ testing methods can lead to more accurate and reliable strength prediction. Among the NDT methods, the most frequent used one is a combination of rebound hammer test and UPV test, which is well known as SonReb. This combination technique is used because the rebound number $(\mathrm{RN})$ obtained by rebound hammer test can provide the information about the concrete strength near to the surface, while UPV can reflect the concrete properties along the thickness of the structural component.

However, SonReb results are affected by the variability in environment (such as temperature and humidity), measurement process (such as rebar effect), and material properties (such as age of concrete, mix proportion, stresses in the concrete, and curing conditions). Reducing the variability can improve the accuracy of the $f_{c}$ predictions.

To account for the variability described above, there are three commonly used 
prediction techniques: computational modeling, artificial intelligence, and parametric multivariable regression models. Computational modeling technique is often based on modeling the complex physical phenomenal, which requires complicated mathematical models. Popovics (1995) gave a review of mathematical models for strength development of Portland cement concrete. Lin et al. (2003) established mathematical models for predicting concrete pulse velocity. However, the computational modeling often is not practical due to the complexity of the model and/or the time-consuming numerical computation. Artificial intelligence, including artificial neural network (ANN) and fuzzy logic method, is a non-parametric statistical tool and is able to consider nonlinear multivariable regressions without knowing the theoretical relationships between the input and the output. Application of ANN and fuzzy logic method to predict $f_{c}$ can be found in many recent publications (Hola and Schabowicz 2005; Cho et al. 2009; Kewalramani and Gupta 2006; Trtnik et al. 2009; Na et al. 2009). However, the relation that obtained by artificial intelligence has no physical meaning but only provides the best fit between the predictions and actual values.

Parametric multivariable regression models, on the other hand, can perform faster than the other two techniques; therefore, it is suitable to be implemented to other applications. Particularly, the confidence interval of prediction calculated by parametric regression models indicates the reliability of the estimation, and the statistics of model parameters can provide insight of how each variable influences the prediction. By far, a considerable number of parametric regression models using SonReb to predict $f_{c}$ have been developed (Samarin and Meynink 1981; Tanigawa et al. 1984; Ramyar and Kol 
1996; Bellander 1979; Hobbs and Kebir 2007; Wiebenga 1968; Arioğlu and Manzak 1991; Sriravindrajah et al. 1988; Arioğlu and Köylüoğlu 1996; Kheder 1999; Arioğlu et al. 1994; Postacioglu 1985); however, these regression models can only be applied to the concrete with the same characteristics of the concrete used for the calibration of the model. Thus, the different suggested formulations of regression models are due to the limited scope of calibration data used. Additionally, the suitability of applying these suggested formulations to the data that is not used for calibration is still not clear. Therefore, a robust regression model based on different type of concrete is needed for the general use. In addition to SonReb measurements (UPV and RN), other variables may be needed in the model to reflect the information about concrete properties to improve the prediction accuracy.

In this study, $f_{c}$ is predicted by a probabilistic multivariable linear regression model with respect to the SonReb measurements, mixture proportions, curing conditions, and age of concrete. The data used for developing the model cover a wide range of concrete properties. Bayesian updating is used to assess the model parameters and also provides a convenient way to update the model when new additional data become available. An all possible subset model selection is used to obtain an accurate, unbiased, and parsimonious model, while it also helps understand how variables contribute to the prediction. Additionally, multiple imputation (MI) is used to deal with missing data such that the data information can be incorporated as much as possible to develop the regression model.

Note that the previously developed multivariable regression models have 
published in various places, thus it seems worthwhile to give a summation and comparison of these models. To be fair in the comparison, the previously developed models are refitted to the calibration data used in the proposed model as well. Then the validation of those models is checked by using diagnostic plots and marginal model plots. Lastly, the validated models and the proposed model in this Section are applied to a group of test data that collected from different research groups to evaluate the performance and robustness of the models.

\subsection{Review of UPV and Rebound Hammer Tests}

The UPV test is performed by using a sending transducer that sends an ultrasonic pulse to the concrete specimen and a receiving transducer where the pulse is received. With the known distance between the sending and receiving transducers and the time that the stress wave generated by the ultrasonic pulse travels through the concrete, UPV of the specimen can be calculated. Then, the elastic properties of the concrete can be estimated. The relation between UPV and dynamic elastic modulus, $E_{d y n}$ that is correlated to $f_{c}$, can be written as

$$
U P V=\sqrt{\frac{(1-\mu) E_{d y n}}{(1+\mu)(1-2 \mu) \rho}}
$$

where $\mu=$ dynamic Poisson's ratio. This formula shown in Eq. (5.1) is derived for elastic homogeneous materials; however, concrete is well known as inelastic and heterogeneous material. Therefore, using UPV alone to predict $f_{c}$ is not reliable (Komloš 1996). 
Rebound hammer test uses the rebound of an elastic mass, striking at the concrete surface. When the plunger of the rebound hammer is pushed against to the surface and the latch is released, the spring-pulled mass rebounds back with a rebound distance. This distance is measured on a scale numbered from 10 to 100 and is recorded as $\mathrm{RN}$. The measurement $\mathrm{RN}$ depends on the energy absorbed during the impact; therefore, it can be considered as an indicator of the concrete strength. Additionally, it is more likely affected by the near-surface layer of concrete.

As pointed out by many researchers (Trtnik 2009; Chung 1978; Phoon et al. 1999), UPV is effected by numerous factors: properties and proportion of the constituent materials, aggregate content and types, age of concrete, the presence of micro-cracks, water content, and stresses in the concrete specimens, surface condition, temperature of concrete, path length, shape and size of specimen, the presence of reinforcement, and etc. The results of rebound hammer test is significantly influenced by smoothness and carbonation conditions of the concrete, moisture conditions of concrete, type of coarse aggregate, type of cement, cement content, age of concrete, and size, shape, rigidity of the test concrete specimen, and etc. (Domingo and Hirose 2009; Proverbio and Venturi 2005). Because of those influence factors described above, using UPV or RN alone is not able to indicate an accurate prediction of $f_{c}$. However, SonReb can improve the prediction because UPV and RN have different sensitivities to the influence factors, thus additive values are brought in (Breysse 2009). 


\subsection{Development of Multivariable Linear Regression Model}

\subsubsection{Formulation of proposed regression model}

The proposed probabilistic multivariable linear regression model can be expressed as

$$
y(\mathbf{x}, \boldsymbol{\Theta})=\sum_{j=0}^{p} \theta_{j} \cdot h_{j}\left(x_{j}\right)+\sigma \varepsilon
$$

where $\quad \boldsymbol{\Theta}=(\boldsymbol{\theta}, \sigma), \quad y(\mathbf{x}, \boldsymbol{\Theta})=$ predictive $\quad f_{c} \quad$ (or a suitable transformation), $\boldsymbol{\theta}=\left\{\theta_{j}\right\}=$ unknown model parameters, $h_{j}\left(X_{j}\right)=$ predictors or suitable transformation function of $x_{j}, p=$ number of predictors, $\mathbf{x}=\left\{x_{j}\right\}=$ selected variables, such as SonReb measurements, mix proportions, and concrete age, $\sigma=$ standard deviation of model error, and $\varepsilon=$ random variable with zero mean and unit variance. The selection of $x_{j}$ is based on the influence factors of $f_{c}$ and SonReb, while it is also restraint by the data collected. Note that the formulation of Eq. (5.2) is based on two assumptions. One is called homoskedasticity assumption, stating that $\sigma$ is a constant and independent of $\mathbf{x}$. The other one is normality assumption which assumes that $\varepsilon$ follows the normal distribution. These two assumptions can be satisfied by using a parameterized family of power transformation (Box and Cox 1964), which define the power transformation of a data vector $Z$ as

$$
Z^{(\lambda)}=\left\{\begin{array}{c}
\left(Z^{\lambda}-1\right) / \lambda \quad \text { if } \lambda \neq 0 \\
\log (Z) \text { if } \lambda=0
\end{array}\right.
$$

The data vector in this study can be $y$ and $x_{j}$ in Eq. (5.2). Diagnostic plots (Sheather 2008) and marginal model plots (Cook and Weisberg 1997) that are shown later can be 
used to check the suitability of the transformation to ensure the validation of the two assumptions.

\subsubsection{Data collection}

The regression model should be developed based on a large number of widely ranging compressive strength values containing the known information about the concrete properties as much as possible. Although a great number of experimental research using SonReb have been conducted, most of the experimental data or the characteristics of concrete are not explicitly shown in the corresponding literatures. In this study, there are a total of 1103 data collected from a wide literature review and the literature is listed in Appendix D.

The data is set into three groups. Group 1 consists of 412 data and is used for the model assessment and the model selection. This group of data has better information in the variables so that the model selection can be conducted to delete those predictors that do not contribute to the prediction significantly. Group 2 with 555 data is used to update the model that developed based on the data in Group 1. This step illustrates how the model parameters can be updated when new additional data is collected. Group 3 with 136 data is collected from four different research groups (Na et al. 2009; Domingo and Hirose 2009; Malhotra and Carette 1980; Knaze and Beno 1984), and is used to check the model performance. Furthermore, the data in Groups 1 and 2 are called training data, and the Group 3 data is called test data. Table 5-1 gives the ranges of variables for 
the training data. Note that there are missing data in some of the variables in the training data. The methods to manage missing data are described in the following section.

Table 5-1. Ranges of variables from database

\begin{tabular}{|c|c|}
\hline Variable & Range \\
\hline concrete compressive strength, $f_{c}, \mathrm{MPa}$ & $6.86-87.61$ \\
\hline rebound number, $R N$ & $12-52$ \\
\hline ultrasonic pulse velocity, $U P V, \mathrm{~km} / \mathrm{s}$ & $2.92-4.93$ \\
\hline unit weight of concrete, weight, $\mathrm{kN} / \mathrm{m}^{3}$ & $20.38-25.00$ \\
\hline water-cement ratio, $w c, \%$ & $20-70$ \\
\hline unit volume of cement, $v c$ & 89-286 \\
\hline unit volume of sand, $v a, \mathrm{~m}^{3}$ & $96-316$ \\
\hline unit volume of gravel, $\mathrm{vg}, \mathrm{m}^{3}$ & $200-496$ \\
\hline fly ash-cement ratio, $F A, \%$ & $0-20$ \\
\hline superplasticiser-cement ratio, $S P, \%$ & $0-3.5$ \\
\hline sand-aggregate ratio, $s a, \%$ & $20-48$ \\
\hline cement-aggregate ratio, $\mathrm{ca}, \%$ & $12-60$ \\
\hline unit weight of water, water, $\mathrm{kN} / \mathrm{m}^{3}$ & $1.22-2.74$ \\
\hline unit volume air content, air $\%$ & $0.5-32$ \\
\hline Slump, sl, cm & $0-24.5$ \\
\hline age of concrete, age, day & $3-730$ \\
\hline curing temperature, $T, \mathrm{C}^{\mathrm{o}}$ & $14-27$ \\
\hline concrete curing condition, curing & $\begin{array}{l}\text { 1: water curing } \\
\text { 2: air curing } \\
\text { 3: curing outdoors }\end{array}$ \\
\hline aggregate type, $T A$ & $\begin{array}{l}\text { 1: river sand and gravel } \\
\text { 2: river sand and crushed gravel } \\
\text { 3: crushed sand and gravel } \\
\text { 4: recycled aggregate }\end{array}$ \\
\hline
\end{tabular}

\subsubsection{Missing data}

In order to use all the data collected from literature, including the ones that have incomplete data in some of the variables, methods that deal with missing data are 
adopted. Rubin (1987) developed the general statistical theory and framework for managing missing data. A review of different methods to handle incomplete data has been given by Allison (2002) and Litter (1992). The conventional methods include listwise deletion, pairwise deletion, dummy variable adjustment, and least square using imputed data. More advance methods include expectation-maximization (EM), direct maximize likelihood method, and multiple imputation (MI). Note that some methods can only be applied for specific missing data mechanism.

The missing data mechanism gives an idea how the missingness is related to other values. If the probability of missing data in one variable (say variable $x_{1}$ ) is complete random, which means the missing data is unrelated to other data in $x_{1}$ or other variables, then the data in $x_{1}$ is called missing completely at random (MCAR). If the probability of missing data in $x_{1}$ is only unrelated to other data in $x_{1}$, then it is called missing at random (MAR). If the data is not MAR, the missing data is nonignorable. In this study, the missing data in the training data are neither provided in the literature nor measured in the experiments. Therefore, it is legitimate to assume the missing mechanism in the training data is at least MAR.

To assess the model with MAR missing data, MI approach is suitable to apply. Compared with other methods, MI gives consistent, asymptotically efficient, and asymptotically normal estimation (Allison 2002). In MI, for example to deal with missing data in UPV, UPV is regressed on $f_{c}$ and other variables $\tilde{\mathbf{x}}$, with the complete data. Thus, the following regression is obtained, 


$$
U P V=\gamma_{0}+\gamma f_{c}+\boldsymbol{\beta} \tilde{\mathbf{x}}+\sigma_{U P V} \varepsilon
$$

where $\gamma_{0}, \gamma$, and vector $\boldsymbol{\beta}$ are the model parameters, and $\sigma_{U P V}$ is the estimated standard deviation of the model error. The imputation is to make random draws from the distribution obtained from Eq. (5.4). Additionally, the imputation can also consider the variability in the model parameters. Typically, the imputation in MI is based on multivariate normal model. However, it has been found that the imputation is still good enough even if some of variables do not have normal distribution (Schafer 1997). For one chain of MI, it continues until the probability distributions of the variables with missing data convergence. After the imputation is complete, the methods for complete data (such as Bayesian updating) can be used to assess the regression model shown in Eq. (5.2). Due to the random imputation, every time the estimation of interests is different. With $m$ separate chains of iterations, the variability across the chains needs to be considered to adjust the standard deviations. For example, the estimated standard deviation of model parameter $\theta_{j}, \sigma_{\theta_{j}}$, can be calculated by

$$
\sigma_{\theta_{j}}^{2}=S_{\theta_{j}, w}^{2}+\frac{m+1}{m} S_{\theta_{j}, b}^{2}
$$

where $S_{\theta_{j}, w}^{2}$, is the average variance within the imputation chains, and $S_{\theta_{j}, b}^{2}$ is the variance from the between imputation chains. As stated in Schafer and Olsen (1998), only three to five imputation chains are sufficient for many applications. Thus three MI chains of iterations are generated in this study. 


\subsubsection{Assessment of proposed regression model}

To explore the correlation of $f_{c}$ with SonReb measurements and other concrete properties, first as many predictors as possible are used in Eq. (5.2). Based on the data in Group 1, a full model is first developed with 15 predictors with variables $U P V, R N$, weight, ca, sa, wc, SP, FA, sl, age, air, TA, water, UPV/RN, and $\log \left(U P V^{4} R N^{3}\right)$. The definition of those predictors can be found in Table 5-1. The first 13 variables are the SonReb measurements and concrete properties. The last two variables are chosen from literatures that are used in regression models suggested by others. They are included so that the proposed full model can be considered general. In order to choose the right transformation, multivariate generalization of the Box-Cox procedure (Box and Cox 1964) is adopted to transform $f_{c}$ and all the predictors simultaneously to joint normality. A Bayesian updating rule (Box and Tiao 1992) is used to estimate the posterior statistics of the model parameters $\theta_{j}$ and $\sigma$ in Eq. (5.2).

The validation of the full model with 15 predictors is first checked with diagnostic plots shown in Figure 5-1. The diagnostic plots (Sheather 2009) include plots of the residuals and of the roots of the standardized residuals vs. the fitted values, a normal Q-Q plot of the standardized residuals, and a plot of standardized residuals vs. the corresponding leverages. The first two diagnostic plots are used to check the homoskedasticity assumption, the normal Q-Q plot is used to check the normality assumption, and the last diagnostic plot can help identify outliners and bad leverage points. As seen in Figure 5-1, the plots of residuals give no discernible pattern, Q-Q 
plots shows the evidence of normality, and no outliners and leverage pointes are detected. Thus, the validation of the full model is ensured. Furthermore, marginal model plots (Cook and Weisberg 1997) are used to check that each variable is modeled correctly in the full model.

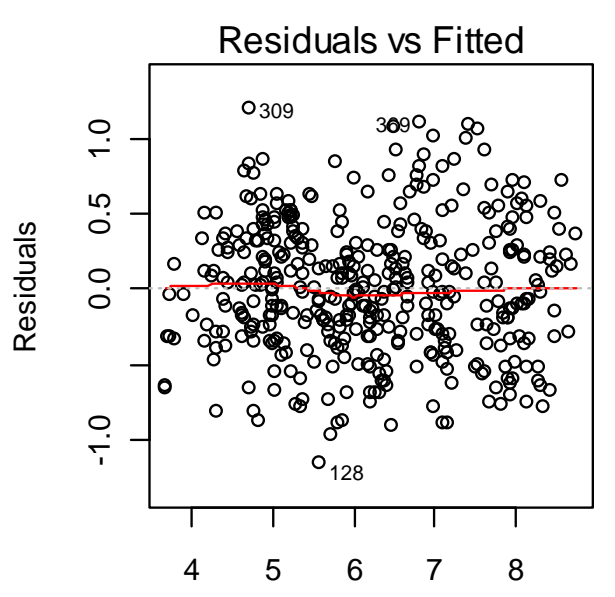

Fitted values

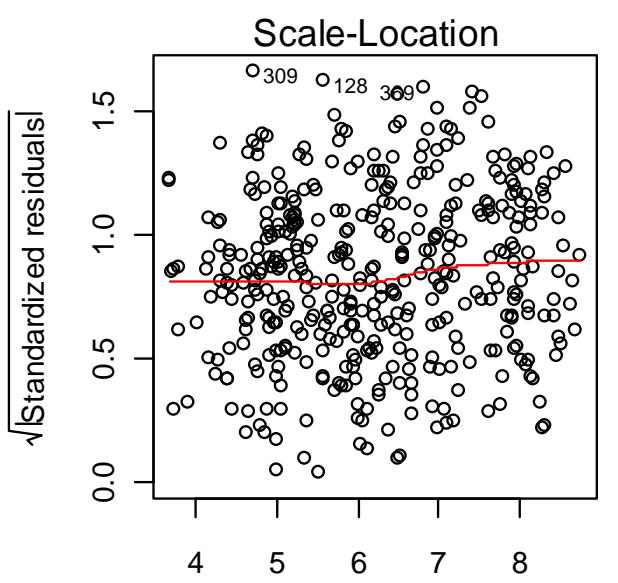

Fitted values
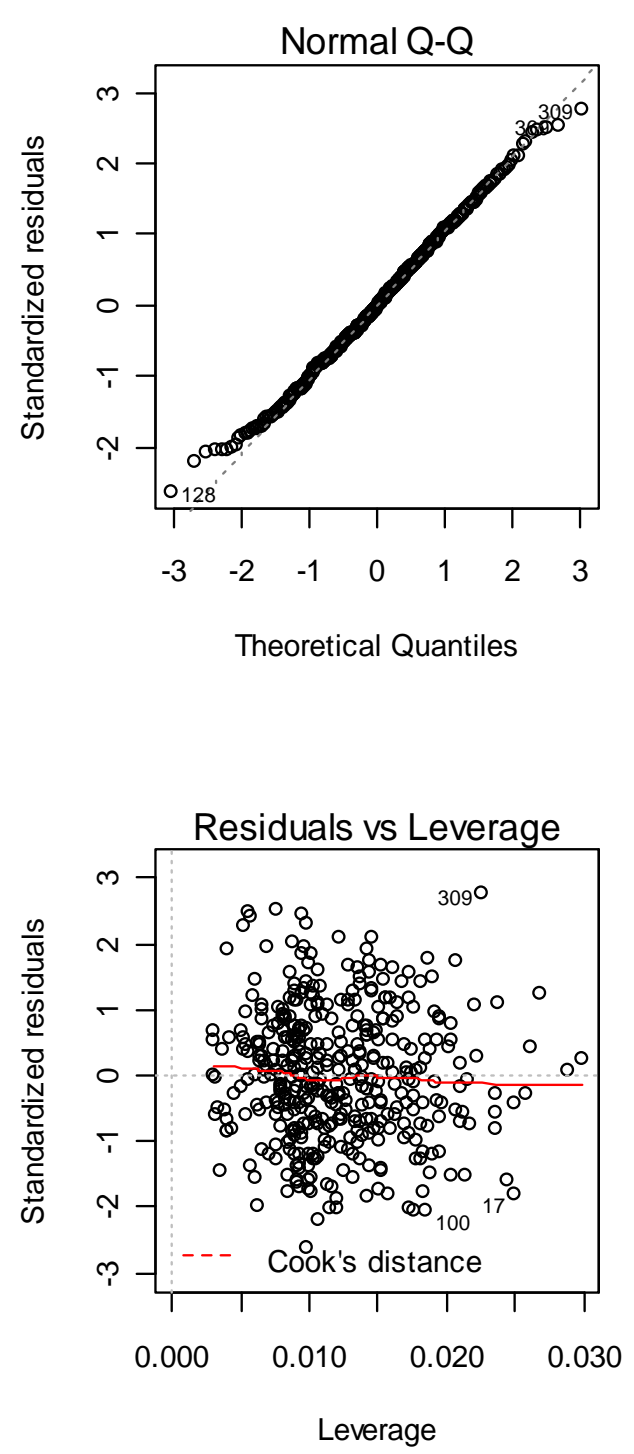

Figure 5-1. Diagnostic plots of the proposed model 
Note that in order to interpret the multivariable regression model, there is an implicit assumption that is the predictors should be interrelated. When strong linear relationship exists among the predictors, there exists a multicollinearity problem that often leads to poor estimations of model parameters. Several methods can be used to detect multicollinearity. One thorough investigation is to exam variance inflation factor, $V I F$, and the VIF $F_{j}$ for predictor $h_{j}\left(x_{j}\right)$ is defined as

$$
V I F_{j}=\frac{1}{1-R_{j}^{2}}
$$

where $R_{j}^{2}=$ square of the correlation coefficient obtained from the regression of $h_{j}\left(x_{j}\right)$ on other predictors. The VIF values exceeding 5 indicate the issue of multicollinearity problem ${ }^{29}$, which is found in the full model in this study.

To manage the multicollinearity in the full model, an all possible subsets model selection process is adopted in this study to remove the redundancy. The use of model selection provides a way to obtain an unbiased and parsimonious model without losing accuracy so that the model is not over- or under-fitting. Moreover, it shows the insight of how variables contribute to the prediction. The all possible subsets model selection uses the adjusted $R^{2}\left(R_{a d j}^{2}\right)$, the Akaike's information criterion ( AIC ) (Akaike 1974), the corrected Akaike's information criterion $\left(A I C_{C}\right)$ (Hurvich and Tsai 1989), and the Bayesian information criterion ( BIC) (Schwarz 1978). The quantity $R_{a d j}^{2}$ is defined as 


$$
R_{a d j}^{2}=1-\frac{\sum_{i=1}^{N_{s}}\left[f_{c p, i}(\mathbf{x}, \hat{\boldsymbol{\Theta}})-f_{c, i}\right]^{2} /\left(N_{s}-N_{p}-1\right)}{\sum_{i=1}^{N_{s}}\left[f_{c p, i} \mathbf{x}, \hat{\boldsymbol{\Theta}}-\bar{f}_{c, i}\right]^{2} /\left(N_{s}-1\right)}
$$

where $f_{c p, i}(\mathbf{x}, \hat{\boldsymbol{\Theta}})=$ predicted value estimated by proposed model with the mean vector of model parameters $\hat{\boldsymbol{\Theta}}, f_{c, i}=$ actual value, $\bar{f}_{c, i}=\sum_{i=1}^{N_{s}} f_{c, i} / N_{s}=$ the sample mean of $f_{c, i}$, $N_{s}=$ number of data, and $N_{p}=$ number of predictors. The criteria of $A I C, A I C_{C}$, and BIC measure how well the model fits the data using the trade-off between the complexity of the model and the model accuracy. They are expressed as

$$
\begin{gathered}
A I C=-2 \ln [L(\boldsymbol{\Theta})]+2 N_{p} \\
A I C_{C}=A I C+\frac{2\left(N_{p}+2\right)\left(N_{p}+3\right)}{N_{s}-N_{p}-2} \\
B I C=-2 \ln [L(\boldsymbol{\Theta})]+N_{p} \ln \left(N_{s}\right)
\end{gathered}
$$

where $L(\boldsymbol{\Theta})=$ the maximum of the likelihood function for the estimated model, and with the two assumptions (homoskedasticity and normality) in the model shown in Eq. (5.2), $L(\boldsymbol{\Theta})$ can be calculated by

$$
L(\boldsymbol{\Theta})=-\frac{N_{s}}{2} \log (2 \pi)-\frac{N_{s}}{2} \log \left(\frac{R S S}{N_{s}}\right)-\frac{N_{s}}{2}
$$

where $R S S=$ residual sum of squares. A higher value of $R_{a d j}^{2}$ and lower values of $A I C$, $A I C_{C}$, and $B I C$ indicates a better model. 
In the all possible subsets model selection, the subset size varies from 1 to 15 in this study, a most desirable model for one subset is identified by maximizing $R_{a d j}^{2}$ and minimizing the other three criteria. Thus 15 potential models, each for one subset, are obtained. Then, the values of $R_{a d j}^{2}, A I C, A I C_{C}$, and BIC are used to choose the best model. Note that maximizing $R_{a d j}^{2}$ and minimizing $A I C, A I C_{C}$, and BIC across all the subsets to select the overall best model can give different answers because of different judging criteria. The test data from Group 3 are also used to check the statistical significance (p-value) of the coefficients of the predictors. The models that have at least one p-value $\geq 0.05$ are considered to be over-fitting the data. Finally, a partial F-test (Sheather 2009) helps to ensure the most parsimonious model.

Table 5-2. Posterior statistics of the parameters in the proposed regression model using Group 1 data

\begin{tabular}{cccccccc}
\hline \multirow{2}{*}{ Parameter } & \multirow{2}{*}{ Mean } & \multirow{2}{*}{$\begin{array}{c}\text { Standard } \\
\text { deviation }\end{array}$} & \multicolumn{6}{c}{ Correlation coefficient } \\
\cline { 5 - 9 } & & $\theta_{0}$ & $\theta_{1}$ & $\theta_{2}$ & $\theta_{3}$ & $\theta_{4}$ \\
\hline$\theta_{0}$ & $-1.85 \mathrm{E}+00$ & $2.42 \mathrm{E}-01$ & 1 & & & & \\
$\theta_{1}$ & $6.63 \mathrm{E}-04$ & $9.29 \mathrm{E}-05$ & 0.71 & 1 & & & \\
$\theta_{2}$ & $2.16 \mathrm{E}-02$ & $1.26 \mathrm{E}-03$ & -0.34 & -0.11 & 1 & & \\
$\theta_{3}$ & $2.98 \mathrm{E}+01$ & $1.79 \mathrm{E}+00$ & -0.87 & -0.88 & -0.01 & 1 & \\
$\theta_{4}$ & $1.42 \mathrm{E}-01$ & $4.14 \mathrm{E}-02$ & -0.64 & -0.53 & -0.09 & 0.55 & 1 \\
$\sigma$ & 0.4412 & - & - & - & - & - & - \\
\hline
\end{tabular}

As a result of applying the model selection, the final formulation of the regression model is obtained 


$$
y=\theta_{0}+\theta_{1} \cdot R N^{2.0}+\theta_{2} \cdot U P V^{3.0}+\theta_{3} \cdot w c^{-0.5}+\theta_{4} \cdot \log (\text { age })+\sigma \varepsilon
$$

where $y=\sqrt{f_{c}}$. Note that the transformations power for $f_{c}$ and the variables are obtained from power transformation followed by the Box-Cox procedure (Box and Cox 1964). Table 5-2 shows the posterior statistics of $\theta_{j}(j=1,2,3,4)$ and $\sigma$. The age of concrete is easily obtainable and the water-cement ratio, $w c$, is usually specified in the construction specification. However, if $w c$ cannot be found, other NDT techniques that are sensitive to $W C$ may need to be incorporated to conduct the prediction.

Table 5-3. Updated posterior statistics of the parameters in the proposed regression model using Group 2 data

\begin{tabular}{cccccccc}
\hline \multirow{2}{*}{ Parameter } & \multirow{2}{*}{ Mean } & \multirow{2}{*}{$\begin{array}{c}\text { Standard } \\
\text { deviation }\end{array}$} & \multicolumn{6}{c}{ Correlation coefficient } \\
\cline { 5 - 9 } & & $\theta_{0}$ & $\theta_{1}$ & $\theta_{2}$ & $\theta_{3}$ & $\theta_{4}$ \\
\hline$\theta_{0}$ & $-3.06 \mathrm{E}+00$ & $1.50 \mathrm{E}-01$ & 1 & & & & \\
$\theta_{1}$ & $2.71 \mathrm{E}-04$ & $4.87 \mathrm{E}-05$ & 0.55 & 1 & & & \\
$\theta_{2}$ & $2.44 \mathrm{E}-02$ & $1.07 \mathrm{E}-03$ & -0.19 & 0.02 & 1 & & \\
$\theta_{3}$ & $3.70 \mathrm{E}+01$ & $1.02 \mathrm{E}+00$ & -0.81 & -0.72 & -0.29 & 1 & \\
$\theta_{4}$ & $2.41 \mathrm{E}-01$ & $1.64 \mathrm{E}-02$ & -0.57 & -0.53 & -0.23 & 0.49 & 1 \\
$\sigma$ & 0.5192 & - & - & - & - & - & - \\
\hline
\end{tabular}

With the developed model, additional data from Group 2 can be easily incorporated to update the model parameters in Eq. (5.12) using Bayesian updating rule. The previous obtained posterior statistics in Table 5-2 now become the prior statistics in this updating. The updated posterior statistics using the data in Group 2 is shown in Table 5-3. The updated posterior means of $\theta_{j}$ are similar with the ones in Table 5-2. The decrease in the updated posterior of standard deviations suggests that the new data 
helps to reduce the statistical uncertainties in the model. Additionally, a little increase in $\sigma$ is reasonable since the model is updated to accommodate the additional data. Figure 5-2 (left) gives the predictions after the Group 2 data is incorporated. With a perfect prediction, the dots should line up along the 1:1 solid line, however, the scatters with the 1:1 line in the Figure 5-2 (left) indicates that the prediction is fairly well nearly without bias. The dashed lines give the region of mean prediction \pm 1 standard deviation.
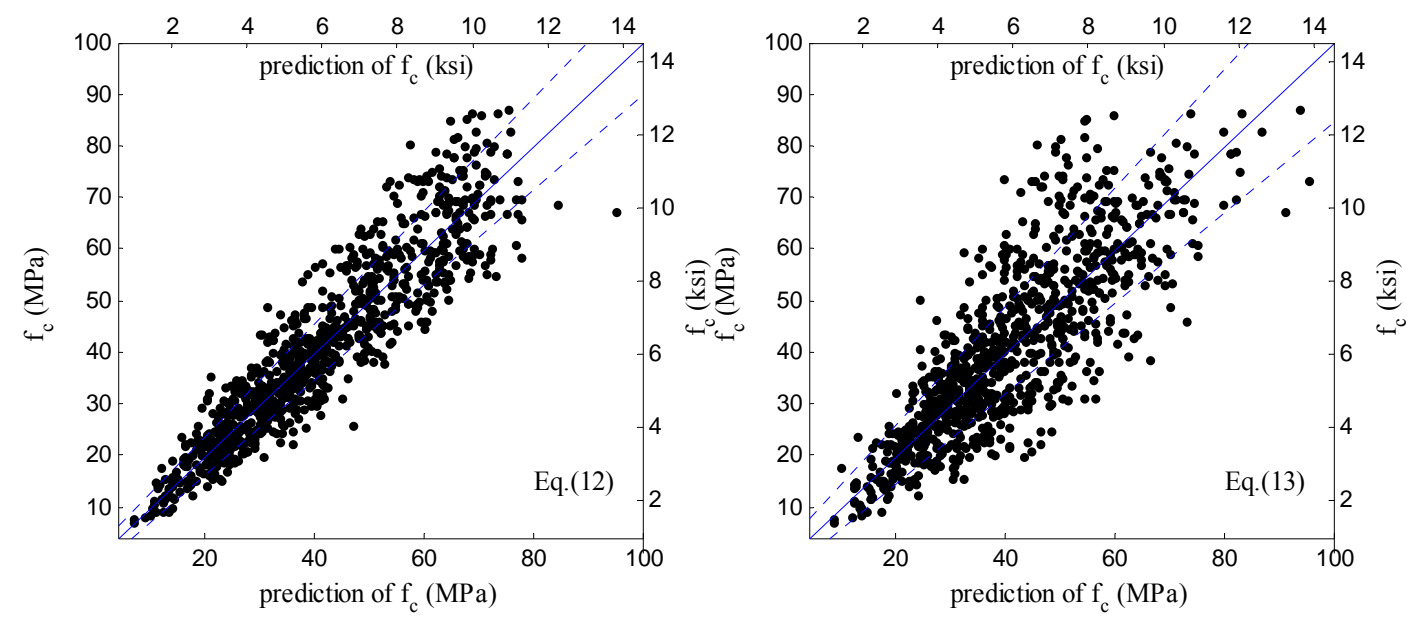

Figure 5-2. Prediction of Eq. (5.12) model (left) and Eq. (5.13) model (right) using training data

Alternatively, the authors also access a regression model with respect to only SonReb measurements for the cases if age of concrete and water-cement ratio cannot be obtained, which is expressed as

$$
y=\theta_{0}+\theta_{1} \cdot R N^{2.0}+\theta_{2} \cdot U P V^{3.0}+\sigma \varepsilon
$$


Table 5-4 gives the statistics of $\theta_{j}(j=1,2,3,4)$ and $\sigma$ for Eq. (5.13). The model error $\sigma$ is increased by about 55\% compared with the regression model shown in Eq. (5.13), indicating that the additional terms, $w c$ and age, greatly reduce the statistical uncertainties in the prediction. This observation can be found in Figure 5-2 (right) as well, where the scatter using Eq. (5.13) is much larger than the one with Eq. (5.12). Accordingly, it further shows that the model selection produces the parsimonious model (Eq. (5.12)) with high accuracy.

Table 5-4. Posterior statistics of the parameters in the regression model shown in Eq. (5.13) using training data

\begin{tabular}{cccccc}
\hline \multirow{2}{*}{ Parameter } & \multirow{2}{*}{ Mean } & $\begin{array}{c}\text { Standard } \\
\text { deviation }\end{array}$ & \multicolumn{4}{c}{$\begin{array}{c}\text { Correlation } \\
\text { coefficient }\end{array}$} \\
\cline { 4 - 7 } & & & $\theta_{0}$ & $\theta_{1}$ & $\theta_{2}$ \\
\hline$\theta_{0}$ & $1.26 \mathrm{E}+00$ & $1.29 \mathrm{E}-01$ & 1 & & \\
$\theta_{1}$ & $1.52 \mathrm{E}-03$ & $4.99 \mathrm{E}-05$ & -0.19 & 1 & \\
$\theta_{2}$ & $3.52 \mathrm{E}-02$ & $1.56 \mathrm{E}-03$ & -0.85 & -0.32 & 1 \\
$\sigma$ & 0.8024 & - & - & - & - \\
\hline
\end{tabular}

\subsection{Evaluation of Proposed Regression Model}

As mentioned previously, it is worthwhile to summarize and check the performance of many different formulations suggested by different investigators. Table 5-5 shows the summary of those different formulations. While the appearances of the formulation seems very different, they all can be considered as linear forms only with different transformations on $f_{c}$ and SonReb measurements. To evaluate the performances of the proposed model in this study and the previously developed models, several comparisons 
are conducted in the following. For the sake of convenience, the proposed model shown in Eq. (5.12) is denoted as M0, and Eq. (5.13) is denoted as $\mathrm{M}_{\mathrm{a}}$.

Table 5-5. A summary of regression formulations developed by different researchers

\begin{tabular}{cc}
\hline Model & Formulation \\
\hline M1 (Tanigawa et al. 1984; Ramyar and Kol 1996) & $f_{c}=t_{0}+t_{1} R+t_{2} U P V$ \\
M2 (Bellander 1979) & $f_{c}=t_{0}+t_{1} R^{3}+t_{2} U P V$ \\
M3 (Samarin and Meynink 1981) & $f_{c}=t_{0}+t_{1} R+t_{2} U P V^{4}$ \\
M4 (Hobbs and Kebir 2007) & $f_{c}=t_{0}+t_{1} R+t_{2} U P V+t_{3} U P V^{2}$ \\
M5 (Wiebenga 1968; Arioğlu and Manzak 1991; & $\log f_{c}=t_{0}+t_{1} R+t_{2} U P V$ \\
Sriravindrajah et al. 1988) & $\log f_{c}=t_{0}+t_{1} \log R+t_{2} \log U P V$ \\
M6 (Arioğlu and Köylüoğlu 1996; & $\log f_{c}=t_{0}+t_{1}\left[\sqrt{\log \left(R^{3} \cdot U P V^{4}\right)}\right]$ \\
Kheder 1999) & $f_{c}^{-0.5}=t_{0}+t_{1} R^{-1}+t_{2}\left(U P V \cdot R^{-1}\right)$ \\
M7 (Arioğlu 1994)
\end{tabular}

To be fair in the comparison, the regression formulations shown in Table 5-5 are refitted using the training data from Groups 1 and 2. Then the diagnostic plots and marginal model plots that have been used to check the proposed model in the previous section are also used to check the validity of the refitted models. Only the valid models should be used to do the further comparison with the proposed model. As a result, M1, M3, M5, and M6 are shown to be valid models, while others are failed in satisfying homoskedasticity and normality assumptions and/or having variable modeled incorrectly. Figure 5-3 shows the predictions obtained from M1, M3, M5, and M6 vs. the true values when the training data is used for calibration. The dashed lines shows mean prediction \pm 1 standard deviation of the prediction for the corresponding model. 

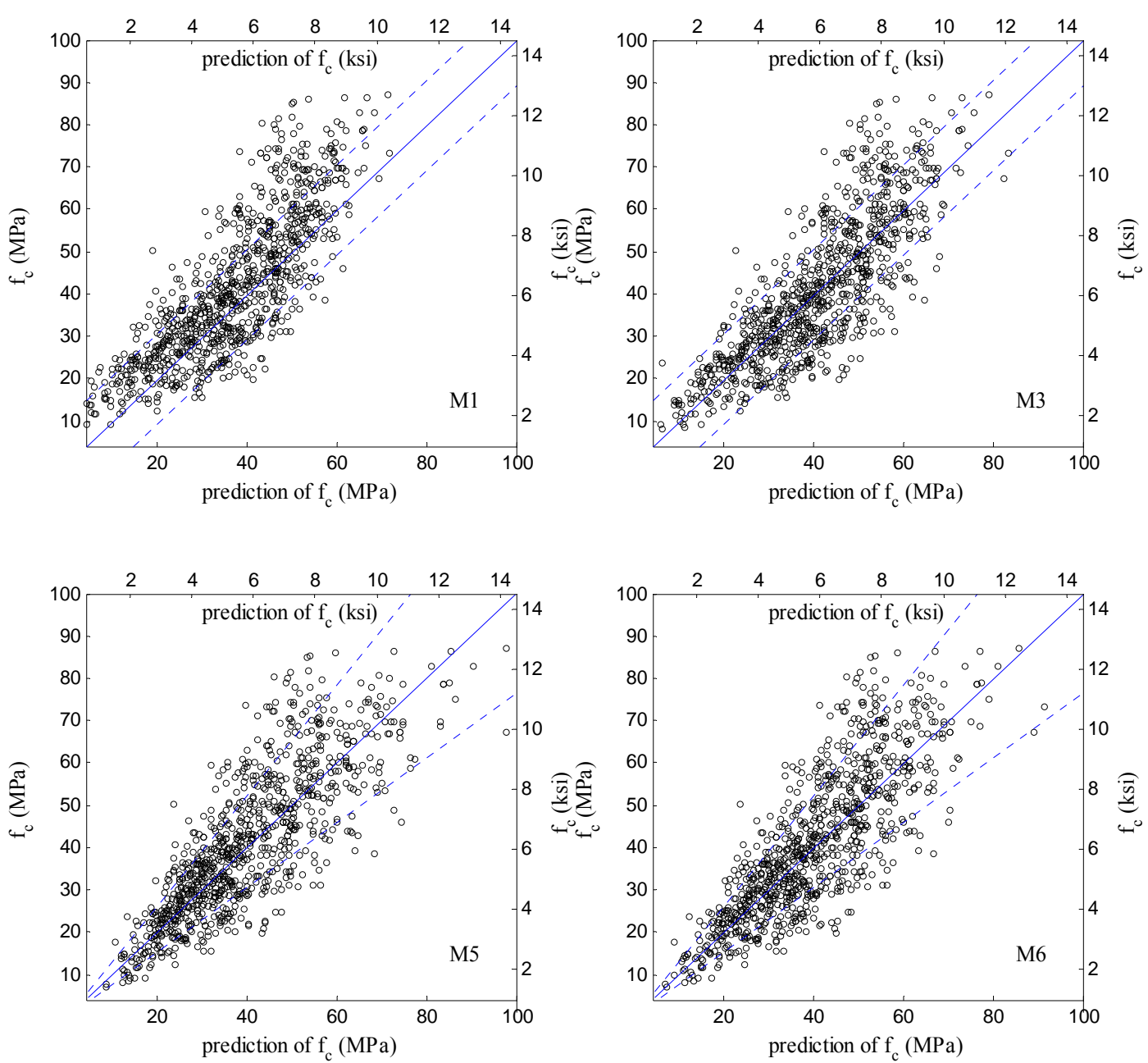

Figure 5-3. Prediction of M1, M3, M5, and M6 suggested by other researchers vs. the true values using training data

Three ways of comparison are conducted in this study. One is to use a measure called Mean Absolute Percentage Error (MAPE), one is to use an evidence ratio (ER) in an $\mathrm{AIC}_{C}$ test, and the other one is to use plots of predictions. MAPE, as an intuitive measure of the accuracy of models, is defined as 


$$
M A P E=\frac{1}{100 N_{s}}\left[\sum_{i=1}^{N_{s}}\left(\frac{\left|f_{c p, i}(\mathbf{x}, \hat{\boldsymbol{\Theta}})-f_{c, i}\right|}{f_{c, i}}\right)\right]
$$

Evidence ratio (Motusky and Christopoulos 2004), ER, provides information about how much more likely the model with lower $A I C_{C}$ to be correct. This ratio is suitable to compare two models when the models are not nested and can be obtained by

$$
E R=e^{0.5 \Delta A I C_{C}}
$$

where $\triangle A I C_{C}=$ absolute difference between $A I C_{C}$ values of two models. The lower ER value is, more likely the model with lower value of $A I C_{C}$ is preferred. The MAPE and ER values for each model are shown in Table 5-6. The proposed model M0 gives the lowest value of MAPE, indicating that M0 is more accurate than others in an average sense. When ER is calculated, it is found M0 has much lower value in $A I C_{C}$ compared with the other four models. Correspondingly the ER values using either training data or test data are extremely large, thus the evidence is overwhelmingly in favor of M0. When the alternative model $\mathrm{M}_{\mathrm{a}}$ is compared with $\mathrm{M} 1, \mathrm{M} 3, \mathrm{M} 5$, and $\mathrm{M} 6$, the prediction obtained by $\mathrm{M}_{\mathrm{a}}$ is not worse if it is not better. When the water-cement ratio and the age of the concrete is not obtainable, $\mathrm{M} 0_{\mathrm{a}}$ is still preferred since the transformations used in $\mathrm{MO}_{\mathrm{a}}$ are the same as $\mathrm{M} 0$ and it has been shown that $\mathrm{M} 0_{\mathrm{a}}$ better satisfies the homoskedasticity and normality assumptions than M1, M3, M5, and M6.

Figure 5-4 shows the comparison of the predictions using M0, M1, M3, M5, and M6 with test data. The region of mean prediction \pm 1 standard deviation of the prediction from the proposed model is shown as dashed lines. It is observed that the 
proposed model gives the best fit to the test data. On the other hand, the models suggested by other researchers tend to overestimate $f_{c}$, even after these models are calibrated and give unbiased prediction for the training data as shown in Figure 5-3. This observation further shows that the proposed model is robust to give much better prediction when using test data that are different from the training data. Additionally, the most predictions by the proposed model are fall into the interval of mean \pm 1 standard deviation (dashed lines in Figure 5-4), indicating a confident estimation.

Table 5-6. A comparison of valid predictive regression models

\begin{tabular}{cccccccccc}
\hline Model & $t_{0}$ & $t_{1}$ & $t_{2}$ & $t_{3}$ & $t_{4}$ & $\begin{array}{c}\text { Training } \\
\text { data }\end{array}$ & $\begin{array}{c}\text { Test } \\
\text { data }\end{array}$ & $\begin{array}{c}\text { Training } \\
\text { data }\end{array}$ & $\begin{array}{c}\text { Test } \\
\text { data }\end{array}$ \\
\hline M0 & -3.06 & 0.00027 & 0.024 & 37.04 & 0.24 & 13.08 & 13.73 & - & - \\
M0 a & 1.26 & 0.00015 & 0.035 & - & - & 19.76 & 27.38 & - & - \\
M1 & -109.09 & 1.32 & 22.52 & - & - & 24.18 & 29.86 & $4.47 \mathrm{E}+201$ & $2.72 \mathrm{E}+24$ \\
M3 & -32.88 & 1.31 & 0.072 & - & - & 21.62 & 32.54 & $7.33 \mathrm{E}+161$ & $4.10 \mathrm{E}+37$ \\
M5 & -0.71 & 0.033 & 0.72 & - & - & 19.91 & 25.52 & $1.02 \mathrm{E}+166$ & $1.91 \mathrm{E}+35$ \\
M6 & -4.66 & 1.06 & 3.07 & - & - & 20.30 & 26.09 & $5.17 \mathrm{E}+164$ & $4.98 \mathrm{E}+30$ \\
\hline
\end{tabular}

\subsection{Conclusions}

In this study, a probabilistic multivariable linear regression model is developed to predict compressive strength, $f_{c}$, using a combined NDT method of ultrasonic pulse velocity (UPV) and rebound number $(\mathrm{RN})$, as known as SonReb. In the model assessment, Bayesian model updating is used to obtain the posterior statistics of model parameters and can be used to update the model parameters when new additional data become available. The model selection that is used to develop unbiased and parsimonious 
models gives us the insight of how the variables contribute to the prediction, instead of treating all variables important as the artificial intelligence does. As a result, additional to SonReb measurements, water-cement ratio and age of concrete are found important and selected in the proposed model. Moreover, this study uses multiple imputation (MI) to handle missing data so that the information that collected from literature is used as much as possible. Overall, this study presents a general guideline for developing a statistically valid multivariable linear regression.

The existence of model error in the proposed formulation is used to capture the inaccuracy of the model form due to NDT measurement errors, environmental effects, and other concrete conditions and properties that are not considered. The simplicity formulation of the proposed model makes it suitable to implement to other applications such as structural reliability analysis.

The combined method, SonReb, is advantageous because UPV and RN have different sensitivities to some parameters that are important to the prediction of $f_{c}$. For example, Trtnik et al. (2009) concluded that aggregate cannot be ignored in order to obtain accurate prediction. However, as suggested in the proposed model that aggregate type is not included because it has significant influence on UPV but not much on RN. Thus, SonReb reduces the number of variables that are needed compared with the case when only one NDT method is used.

In the model performance evaluation, test data is used to compare the prediction of the proposed model and the predictions of four regression models suggested by other researchers. It is concluded that the prediction of the developed model performs best 
either for the training data and the test data. This conclusion further shows that the general guideline proposed in this study can develop a well-performed model.
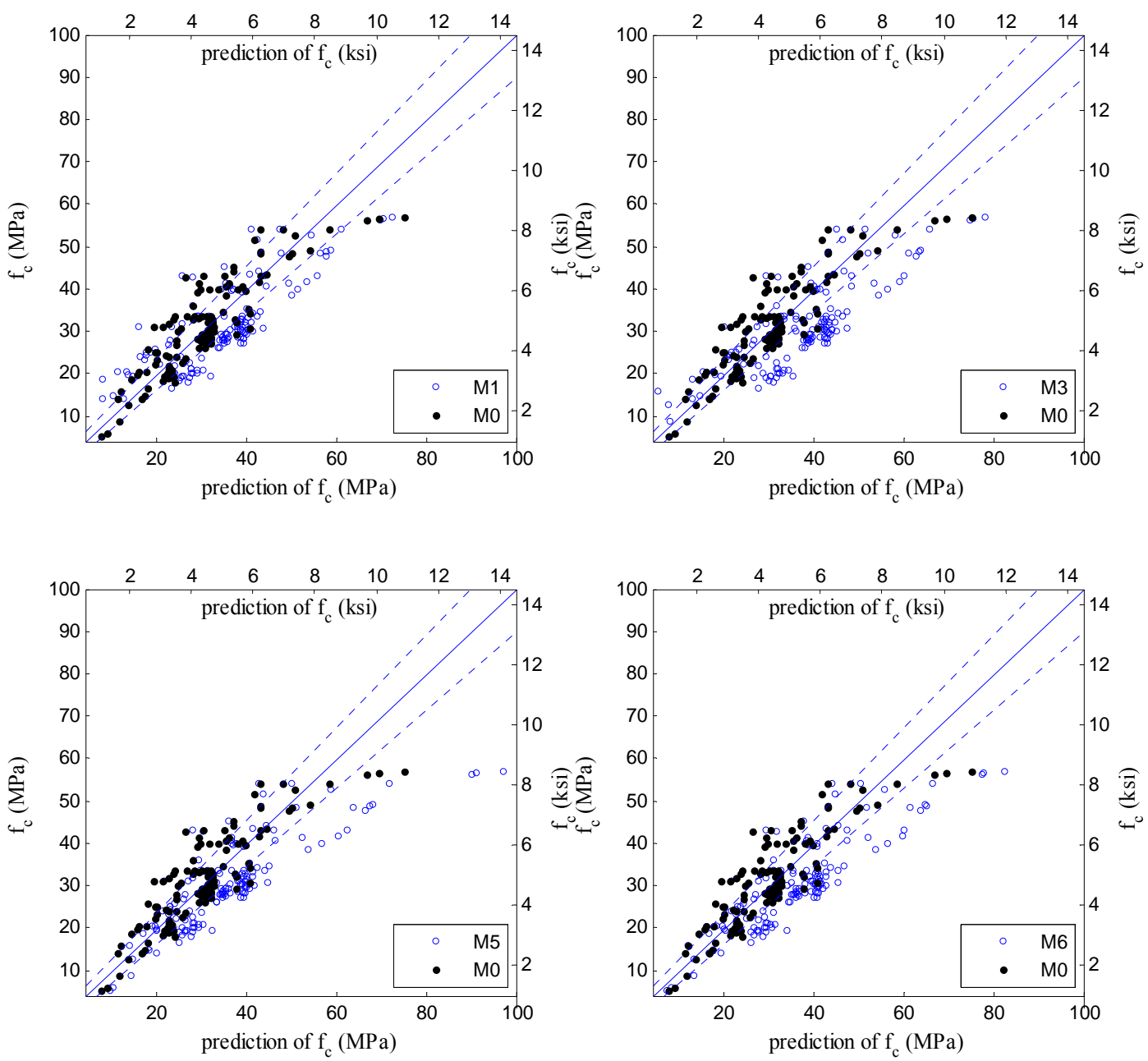

Figure 5-4. Comparison of predictions of compressive strength using different regression models vs. the true values 


\section{CASE STUDY}

\subsection{Introduction}

Incorporating in-place structural properties to the reliability analysis can give more accurate estimates of the actual performance of the bridge. However, limited study has been done in this area. Estes and Frangopol (2003) and Estes et al. (2003) proposed to update the reliability of a bridge based on the results from visual inspections, targeting some specific limitations of deterioration models. Zhao et al. (1994), Byers et al. (1997), and Zhang and Mahadevan (2000) incorporated the information from NDT to fatigue reliability. Zheng and Ellingwood (1998a; b) used the results from NDT to update the distribution of the crack size, considering the interaction of corrosion and fatigue/fracture damage. These research efforts are limited to corrosion and/or fatigue induced deterioration. However, for an existing bridge, besides corrosion and/or fatigue, many other causes can lead to the deterioration. Peil and Mehdianpour (1999) and Peil (2003) described an approach to predict directly the remaining life of a bridge based on monitoring data. However, this is only an empirical approach and it does not use rules of mechanics.

The previous sections describe a framework to identify the actual conditions of a bridge in the field using global and local NDT methods and then to use the results from NDT to estimate the reliability of a existing RC bridge condition in the seismic zones. The $\mathrm{RC}$ bridge reliability is evaluated by seismic fragilities. Seismic fragility is defined as the conditional probability of the seismic demand attaining or exceeding a specified 
capacity given certain earthquake intensity levels, $\mathbf{S}$. The framework can be illustrated by the flowchart shown in Figure 6-1.

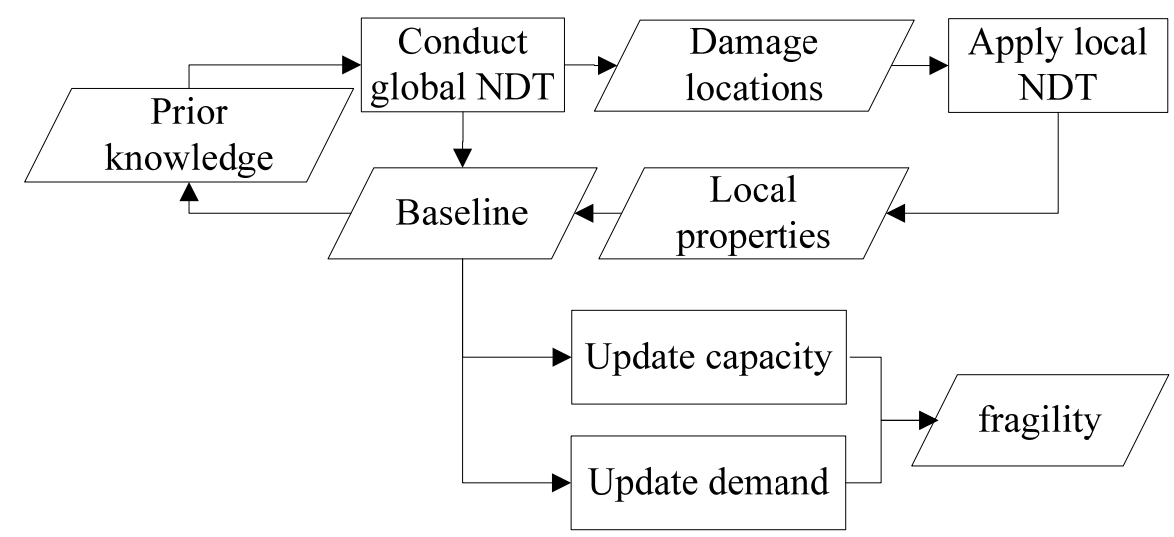

Figure 6-1. Flowchart of the proposed fragility estimate using NDT

\subsection{Case Study}

\subsubsection{Introduction of the numerical bridge}

In this Section, a numerical bridge which is a 3D FEM build in OpenSees (McKenna and Fenves 2000) is used to illustrate the proposed framework that is shown in Figure 6-1. The numerical model simulates a typical box-girder RC highway bridge with one singlecolumn bent and a pile foundation. This bridge configuration is shown in Figure 3-1. In the FEM, the box-girder superstructure is modeled by elastic beam elements with a total of 73 elements (numbered from left to right). The column is modeled using non-linear beam-column elements with a total of 20 elements (numbered from bottom to top) using fiber cross sections including P- $\Delta$ effects. Seat-type abutment is assumed in the longitudinal direction. The interactions between the abutment and the soil are modeled using elastic spring elements that follow the model developed by Maroney et al. (1994). 
It is assumed an initial gap of $152.4 \mathrm{~mm}$ in the longitudinal direction and no gap in the transverse direction. The interaction between the pile and the soil is modeled using bilinear elastic-perfectly plastic springs. The stiffness for the pile springs varies with different soil types. Mackie and Stojadinović (2003) give the properties of springs based on the USGS (U.S. Geological survey) soil classification.

Table 6-1. Design parameters for the preliminary (identified) FEM of the bridge

\begin{tabular}{|c|c|c|c|}
\hline Design Parameter & Value or Mean & $\mathrm{COV}$ & Distribution \\
\hline Degree of skew, $\alpha$ & $0^{\circ}$ & - & - \\
\hline Span (the shorter one), $L_{1}$ & $30.480 \mathrm{~m}$ & $1 \%$ & Lognormal \\
\hline Column height, $H_{c}$ & $6.706 \mathrm{~m}$ & $1 \%$ & Lognormal \\
\hline Reinforcement nominal yield strength, $f_{y}$ & $344.74 \mathrm{MPa}$ & $5 \%$ & Lognormal \\
\hline Transverse reinforcement nominal yield strength, $f_{y h}$ & $275.79 \mathrm{MPa}$ & $5 \%$ & Lognormal \\
\hline Concrete compressive strength of deck, $f_{c, d}$ & $\begin{array}{c}34.47 \mathrm{MPa} \\
(40.17 \mathrm{MPa})\end{array}$ & $7.7 \%^{\dagger}$ & Lognormal \\
\hline Concrete compressive strength of column, $f_{c, c}$ & $\begin{array}{c}34.47 \mathrm{MPa} \\
(43.72 \mathrm{MPa})\end{array}$ & $21.4 \%{ }^{\dagger}$ & Lognormal \\
\hline $\begin{array}{l}\text { Square root of concrete compressive strength of column } \\
\text { Elements } 15 \text { and } 16, \sqrt{f_{c, \text { dam }}}\end{array}$ & $\begin{array}{l}5.87 \mathrm{MPa} \\
(5.49 \mathrm{MPa})\end{array}$ & $\begin{array}{c}0.9 \% \\
13.9 \%\end{array}$ & Normal \\
\hline Longitudinal reinforcement ratio (column), $\rho_{l}$ & $3.59 \%$ & - & - \\
\hline Transverse reinforcement ratio (column), $\rho_{s}$ & $1.06 \%$ & - & - \\
\hline Additional bridge dead load, $w_{t}$ & $10 \%$ self-weight & $25 \%$ & Normal \\
\hline Pile soil stiffness, $K_{\text {soil }}$ & (USGS) C & - & - \\
\hline Abutment stiffness in the trans. direction, $K_{a b u t, t}$ & $\begin{array}{l}88.42 \mathrm{kN} / \mathrm{mm} \\
85.28 \mathrm{kN} / \mathrm{mm}\end{array}$ & $3.4 \%{ }^{\dagger}$ & Normal \\
\hline Two-span ratio, $L_{2} / L_{1}$ & 1.25 & - & - \\
\hline Column concrete cover, cover & $0.038 \mathrm{~m}$ & $10 \%$ & Lognormal \\
\hline
\end{tabular}

${ }^{\dagger}$ Identified from Bayesian model updating

*Identified using SonReb with regression model Eq. (4.12)

${ }^{\dagger}$ Identified using SonReb with regression model Eq. (4.13)

With the information described above, the design parameters for the preliminary 
FEM are given in Table 6-1. A target baseline is constructed the same way as preliminary FEM but with different values in $f_{c, d}, f_{c, c}$, and $K_{a b u t, t}$. The ratios between the preliminary values and the values in target baseline are shown in Table 6-2. Furthermore, local damages are introduced into this target baseline. In this illustration, one damage scenario is studied which is simulated by reducing $f_{c}$ of column Elements 15 and 16 by $20 \%$, i.e., their equivalent flexural stiffnesses are reduced about $10 \%$. The compressive strength of Elements 15 and 16 are denoted by $f_{c, d a m}$. The FEM with reduced local column elements can be considered as a selected damaged bridge in the field. Note that in reality, the structural properties of the target baseline and the damaged FEM are unknown. The values of $f_{c, d}, f_{c, c}, K_{a b u t, t}$ in the target baseline are chosen to be different from the preliminary FEM so that how they can be identified from preliminary values using NDT can be illustrated. The local damage is introduced in the damaged FEM so that the application of global NDT to find damage locations and the application of local NDT to identify the local characteristics of structural properties can be illustrated.

Table 6-2. Parameter ratios between the baseline values and the preliminary values

\begin{tabular}{ccccc}
\hline \multirow{2}{*}{ Parameters } & $\begin{array}{c}\text { Preliminary } \\
\text { FEM }\end{array}$ & \multirow{2}{*}{$\begin{array}{c}\text { Target } \\
\text { baseline }\end{array}$} & \multicolumn{2}{c}{ Identified baselines } \\
\cline { 4 - 5 } & & 1.2 & mean & std. \\
\hline$\left(f_{c, d}\right)_{b} /\left(f_{c, d}\right)_{p}$ & 1.0 & 1.2589 & 0.2690 \\
$\left(f_{c, c}\right)_{b} /\left(f_{c, c}\right)_{p}$ & 1.0 & 1.2 & 1.1560 & 0.0886 \\
$\left(K_{a b u t, t}\right)_{b} /\left(K_{a b u t, t}\right)_{p}$ & 1.0 & 1.5 & 1.5069 & 0.0505 \\
\hline
\end{tabular}




\subsubsection{Applying global NDT}

\subsubsection{Conduct a vibration test on the damaged FEM}

A vibration test is conducted first on the damaged FEM by applying a pulse force at node 15 of the bridge deck. The pulse force is applied in $\mathrm{X}, \mathrm{Y}$, and $\mathrm{Z}$ three directions so that the bridge modes in different directions can be excited. The acceleration responses are recorded at every 6 nodes on the deck and every other node on the column, which simulates 13 accelerometers are evenly put on the deck and 10 accelerometers on the column. To simulate the measurement error, Gaussian white noises with zero means and the standard deviations equal to $1 \%$ of the amplitude of the acceleration responses is used. Furthermore, $10 \mathrm{~s}$ is used as the recording time and $0.005 \mathrm{~s}$ is used as the sampling time. Such vibration test is repeated 10 times, and thus 10 sets of responses are recorded. 
Mode $1: \mathrm{z}_{1}$

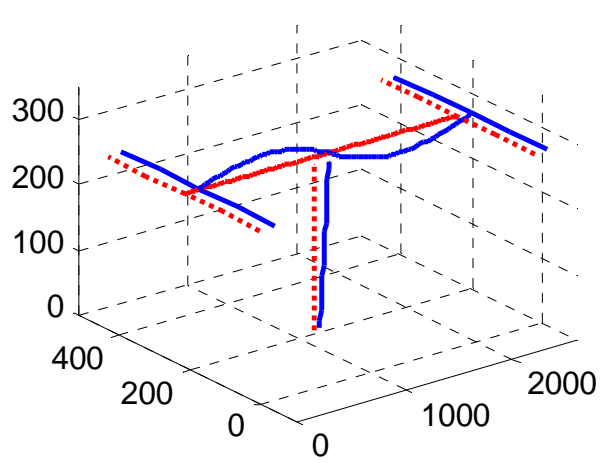

Mode 3: $\mathrm{z}_{2}$

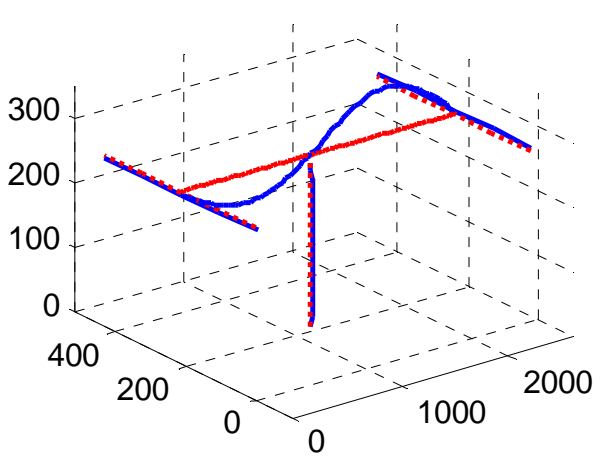

Mode 5: $z_{3}$

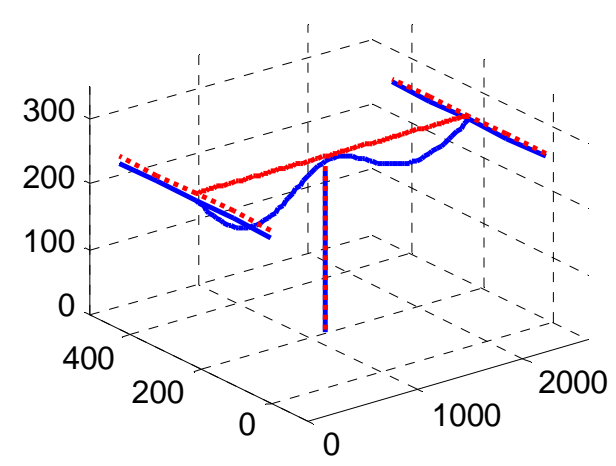

Mode 2: $\mathrm{y}_{1}$

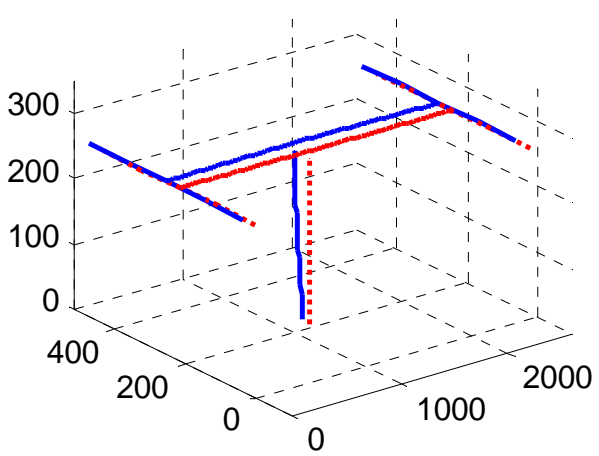

Mode 4: $y_{2}$

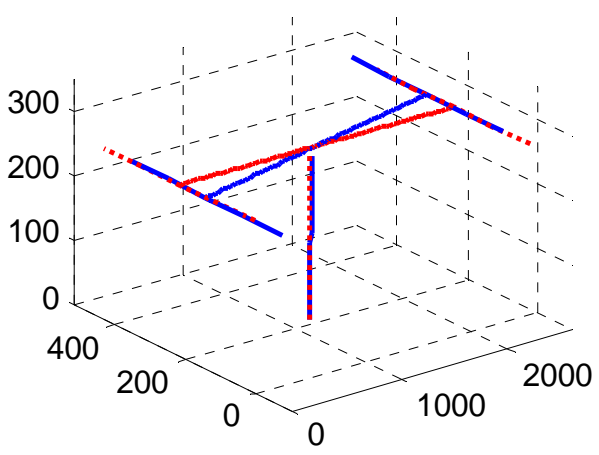

2000

Figure 6-2. First five mode shapes for the example bridge 


\subsubsection{Determine measurement error and modeling error in TDD}

Following the modal parameter identification method, TDD (Kim et al. 2005), 10 sets of modal parameters can be extracted from the 10 sets of acceleration responses. Note that the errors in the extracted modal parameters come from two sources: one is propagated from the measurement error in the vibration tests, and the other one is due to the modeling error in TDD process. Section 4 gives the details of accounting for the effects due to these two errors. To assess the effect due to the measurement error, 1000 sets of acceleration responses at the same measurement noise level are generated using a bootstrap method based on the 10 sets of recorded responses. To assess the modeling error due to TDD, 400 FEMs with the same configurations as the preliminary FEM are generated by varying $f_{c, d}, f_{c, c}, K_{a b u t, t}$. The reason for choosing the sampling size of 400 is discussed in Section 4. In this study, the first five modes are used and they are first three deck modes in $Z$ directions $\left(i=z_{1}, z_{2}, z_{3}\right)$ and first two deck modes in $Y$ directions $\left(i=y_{1}, y_{2}\right)$, as shown in Figure 6-2. Here $e_{f_{i}}$ and $\mathbf{e}_{\boldsymbol{\varphi}_{i}}$ denote the modeling errors in the $i$ th modal frequencies and mode shape due to the TDD process. The mean and standard deviation of $e_{f_{i}}, \mu_{e f}$ and $\sigma_{e f}$, are found as shown in Table 6-3.

Table 6-3. Estimate of modeling errors in modal frequencies

\begin{tabular}{cccccc}
\hline \multirow{2}{*}{ Parameter } & \multicolumn{5}{c}{ Mode } \\
\cline { 2 - 6 } & $z_{1}$ & $z_{2}$ & $z_{3}$ & $y_{1}$ & $y_{2}$ \\
\hline Mean error $(\mathrm{Hz})$ & 0.0011 & 0.0038 & 0.0017 & 0.0039 & -0.0027 \\
Std. deviation of error $(\mathrm{Hz})$ & 0.0212 & 0.0160 & 0.0110 & 0.0179 & 0.0110 \\
\hline
\end{tabular}


Note that in this specific illustration, the mode shapes of the column are of interest. Following the procedures in Section 4, the linear regression models for the modeling errors of the column mode shapes are found as,

$$
\begin{gathered}
\mathbf{e}_{\boldsymbol{\varphi}, z 1}=\alpha_{1, z 1} \boldsymbol{\varphi}_{z 2}+\beta_{1, z 1}\left(f_{z 2}-f_{z 1}\right)^{-1} \boldsymbol{\varphi}_{z 2}+\left(\sigma_{0, z 1} \mathbf{I}+\sigma_{1, z 1}\left|\boldsymbol{\varphi}_{z 2}\right|\right) \varepsilon+\sigma_{z 1} \boldsymbol{\varepsilon} \\
\mathbf{e}_{\boldsymbol{\varphi}, z 2}=\alpha_{1, z 2} \boldsymbol{\varphi}_{z 1}+\left(\sigma_{0, z 2} \mathbf{I}+\sigma_{1, z 2}\left|\boldsymbol{\varphi}_{z 1}\right|\right) \varepsilon+\sigma_{z 2} \boldsymbol{\varepsilon} \\
\mathbf{e}_{\boldsymbol{\varphi}, y 1}=\alpha_{1, y 1} \boldsymbol{\varphi}_{y 2}+\left(\sigma_{0, y 1} \mathbf{I}+\sigma_{1, y 1}\left|\boldsymbol{\varphi}_{y 2}\right|\right) \varepsilon+\sigma_{y 1} \boldsymbol{\varepsilon} \\
\mathbf{e}_{\boldsymbol{\varphi}, y 2}=\alpha_{1, y 2} \boldsymbol{\varphi}_{y 1}+\left(\sigma_{0, y 2} \mathbf{I}+\sigma_{1, y 2}\left|\boldsymbol{\varphi}_{y 1}\right|\right) \varepsilon+\sigma_{y 2} \boldsymbol{\varepsilon}
\end{gathered}
$$

where $\alpha, \beta$, and $\sigma$ are the model parameters that are given in Table $6-4, \mathbf{I}=$ a vector with all one entries, $\varepsilon=$ normal random variables with zero mean and unit variance, and $\boldsymbol{\varepsilon}=$ a vector of normal random variables with zero mean and unit variance. Additionally, the modeling error for the mode shape of mode $i=z_{3}$ is not assessed because the column deformation is in the axial direction for this mode, which does not reflect the flexural stiffness of the column. Thus it has no contribution in DIM to detect the damage locations on the column.

\subsubsection{Identify baseline using Bayesian model updating}

The next step of the global NDT is to update the preliminary FEM with the modal frequencies obtained from the vibration test on the damaged FEM. Table 6-5 compares the mode frequencies of the preliminary FEM, the target baseline, the identified baseline, and the damaged FEM. The differences between the values in the preliminary FEM and the ones in the damaged FEM indicate that preliminary FEM needs to be updated. The 
chosen parameters should be sensitive to the vibration measurement data and enable the correction of the recognized uncertainties (Friswell and Mottershead, 1995). With the mode frequencies used for the model updating, the posterior PDF of the chosen parameters $\mathbf{x}_{m}=\left\{f_{c, d}, f_{c, c}, K_{a b u t, t}\right\}, p^{\prime}\left(\mathbf{x}_{m}\right)$, can be obtained by Bayesian model updating through

$$
p^{\prime}\left(\mathbf{x}_{m}\right)=\kappa L\left(\mathbf{F} \mid \mathbf{x}_{m}\right) p\left(\mathbf{x}_{m}\right)
$$

where $\kappa=\left[\int L\left(\mathbf{F} \mid \mathbf{x}_{m}\right) p\left(\mathbf{x}_{m}\right) \mathrm{d} \mathbf{x}_{m}\right]^{-1}, p\left(\mathbf{x}_{m}\right)=$ prior PDF of $\mathbf{x}_{m}$, and $L\left(\mathbf{F} \mid \mathbf{x}_{m}\right)=$ likelihood function and can be calculated by

$$
L\left(\mathbf{F} \mid \mathbf{x}_{m}\right)=\frac{1}{\prod_{j}\left(2 \pi \cdot \sigma_{f, j}\right)} \exp \left\{\sum_{j} \frac{\left[\left(\hat{f}_{j}^{2}-f_{j}^{2}\right) / f_{j}^{2}-\mu_{f, j}\right]^{2}}{-2 \sigma_{f, j}{ }^{2}}\right\}
$$

where it assumes that the prediction error, $\xi_{j}=\left(\hat{f}_{j}^{2}-f_{j}^{2}\right) / f_{j}^{2}$, between the square of the frequency predicted using FEM, $\hat{f}_{j}$, and the square of the corresponding target frequency, $f_{j}$, follows a normal distribution. The target frequencies are the modal frequencies extracted from the vibration data obtained from the vibration test on the damaged FEM. The target frequencies for the Bayesian model updating is the extracted frequencies from the vibration data obtained from a vibration test on the damaged FEM. In Eq. (6.5), one could use a noninformative prior that is a locally uniform distribution (Box and Tiao 1992). As mentioned before, using the modal frequencies can help construct an accurate baseline because damage at local level has insignificant effects on the modal frequencies, 
which can be confirmed by the closeness between the modal frequencies of the target baseline and the ones of the damaged FEM as shown in Table 6-5.

Table 6-4. Statistics of the model parameters

\begin{tabular}{ccccccc}
\hline & & $\alpha_{1}$ & $\beta_{1}$ & $\sigma_{0}$ & $\sigma_{1}$ & $\sigma$ \\
\hline \multirow{2}{*}{$z_{1}$} & mean & $-9.93 \mathrm{E}-03$ & $8.63 \mathrm{E}-03$ & $4.19 \mathrm{E}-05$ & $1.28 \mathrm{E}-03$ & $1.63 \mathrm{E}-09$ \\
& $\operatorname{cov}^{\dagger}$ & $-1.93 \mathrm{E}-02$ & $2.19 \mathrm{E}-02$ & - & - & - \\
\hline \multirow{2}{*}{$z_{2}$} & $\begin{array}{c}\text { mean } \\
2\end{array}$ & $2.54 \mathrm{E}-03$ & - & $1.23 \mathrm{E}-04$ & $3.05 \mathrm{E}-03$ & $1.43 \mathrm{E}-08$ \\
& $\operatorname{cov}^{\dagger}$ & $1.56 \mathrm{E}-02$ & - & - & - & - \\
\hline \multirow{2}{*}{$y_{1}$} & mean $_{1}$ & $1.41 \mathrm{E}-03$ & - & $1.44 \mathrm{E}-06$ & $1.32 \mathrm{E}-03$ & $3.72 \mathrm{E}-10$ \\
& $\operatorname{cov}^{\dagger}$ & $1.06 \mathrm{E}-02$ & - & - & - & - \\
\hline \multirow{2}{*}{$y_{2}$} & mean & $-3.00 \mathrm{E}-03$ & - & $1.53 \mathrm{E}-05$ & $1.60 \mathrm{E}-03$ & $1.10 \mathrm{E}-09$ \\
& $\operatorname{cov}^{\dagger}$ & $-5.79 \mathrm{E}-03$ & - & - & - & - \\
\hline
\end{tabular}

$\dagger \operatorname{cov}=$ coefficient of variation $=$ standard deviation $/$ mean

Table 6-5. Comparison of modal frequencies $(\mathrm{Hz})$

\begin{tabular}{cccccc}
\hline \multirow{2}{*}{ Mode } & $\begin{array}{c}\text { Preliminary } \\
\text { FEM }\end{array}$ & $\begin{array}{c}\text { Target }^{\dagger} \\
\text { baseline }\end{array}$ & $\begin{array}{c}\text { Damaged } \\
\text { FEM }^{*}\end{array}$ & \multicolumn{2}{c}{ Identified baseline } \\
\cline { 5 - 6 }$z_{1}$ & 2.4752 & 2.5009 & 2.5415 & 2.5049 & 0.0221 \\
$z_{2}$ & 3.2977 & 3.4484 & 3.4213 & 3.4309 & 0.0566 \\
$z_{3}$ & 4.7685 & 4.9970 & 4.9853 & 4.9673 & 0.0886 \\
$y_{1}$ & 2.3623 & 2.7673 & 2.7370 & 2.7759 & 0.0393 \\
$y_{2}$ & 3.8474 & 4.6464 & 4.6921 & 4.6517 & 0.0616 \\
\hline
\end{tabular}

frequencies obtained from modal analysis

${ }^{\ddagger}$ frequencies obtained from TDD

As the results of the model updating, the modal frequencies from the target baseline agree well with the identified baseline as found in Table 6-5. Similarly, the updated parameters ratios from identified baseline come to an agreement with the ones in the target baseline shown in Table 6-2. Those observations conclude that the 
preliminary FEM has been updated successfully.

\subsubsection{Identify damage locations by DIM}

With the mode shapes from the identified baseline, and mode shapes extracted from the vibration data obtained from a vibration test on the damaged FEM, DIM is applied to identify the damage locations on the column. If the column is divided into small segments along the height (in this case, 10 segments with the same length are divided), for the $i$ th segment, DIM calculates a corresponding damage index, $D I_{i}$. Then, a corresponding normalized damage index can be calculated by $Z_{i}=\left(D I_{i}-\mu_{D I}\right) / \sigma_{D I}$, where $\mu_{D I}$ and $\sigma_{D I}$ refer to the mean and standard deviation of $D I_{i}$. To identify the damage location, a threshold value needs to be selected. When a threshold $\lambda_{i}$ is chosen, the probabilities that the DIM indicates damage $\left(I D_{i}\right)$ and does not indicating damage $\left(N I D_{i}\right)$ in a component $i$ can be defined as,

$$
\begin{gathered}
P\left(I D_{i}\right)=P\left(Z_{i} \geq \lambda_{i}\right) \approx \frac{\# \text { of cases where } Z_{i} \geq \lambda_{i}}{n} \\
P\left(N I D_{i}\right)=P\left(Z_{i}<\lambda_{i}\right) \approx \frac{\# \text { of cases where } Z_{i} \geq \lambda_{i}}{n}
\end{gathered}
$$

where $n=$ number sets of mode shapes that are extracted from vibration tests. As described before, there are 1000 sets of acceleration responses at the same measurement noise level are generated, which gives 1000 sets of mode shapes for the damaged FEM. Thus, $n=1000$ in this numerical study. 
Figure 6-3 gives the damage index of each segment for the simulated damage case under measurement noise level of $1 \%$, considering the measurement error and modeling error in TDD. The variability of the damage index as shown in Figure 6-3 indicates that the influences of the modeling and measurement errors on DIM are not negligible. To determine probability of damage detection, three different threshold values are used, as shown in Figure 6-4. As discussed in Section 4, a lower value of threshold gives a higher probability of correct detection for the damaged elements but also higher probability of false detection for the undamaged elements. On the other hand, a higher value of threshold lowers the false detection of undamaged elements but also the correct detection of damaged elements. Comparing the results in Figure 6-4 with the results in Section 4, one can find that with the same level of measurement noise and about the same level of damage severity, the probability of the correct detection for the damaged elements is much lower for this illustration even when the threshold is low. This is due to the fact that in DIM, the structural component is assumed as EulerBernoulli beam; however, the bridge column in this study has almost 4:1 slender ratio which may not quite well satisfy the assumption of an Euler-Bernoulli beam. Again, as found in Section 4, for the undamaged element adjacent to the end of the column, the probability of false detection is high, which is partially due to the use of spline interpolation for calculating the mode shape curvature in DIM. 


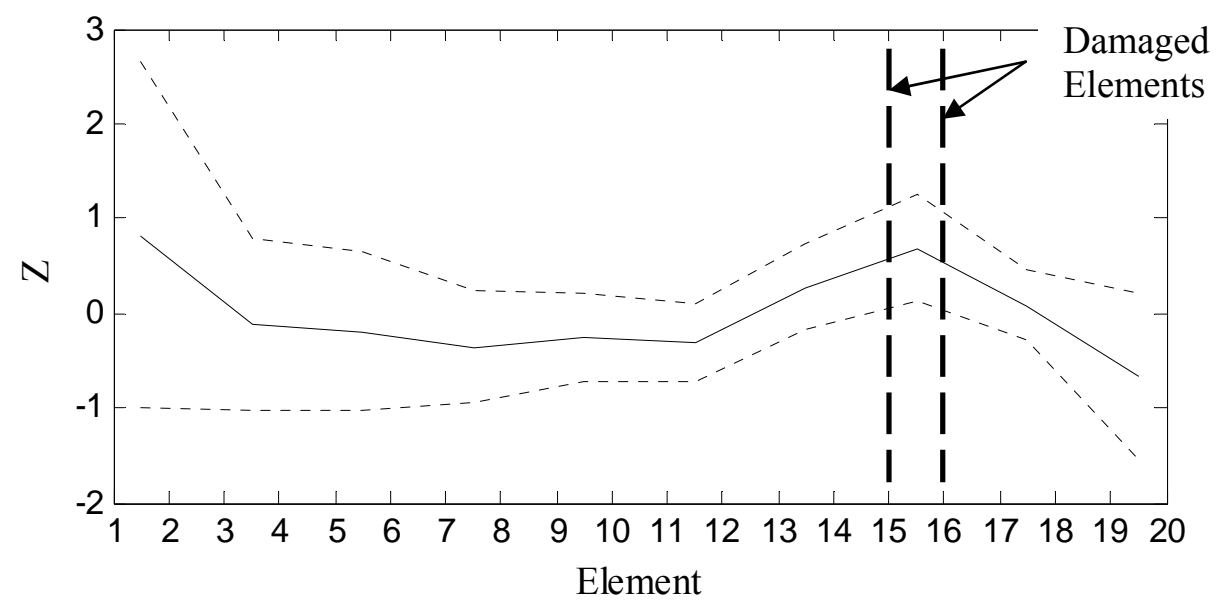

Figure 6-3. $Z$ values (solid line: mean, dotted line: mean \pm 11 standard deviation) for the bridge column elements with under $1 \%$ measurement error

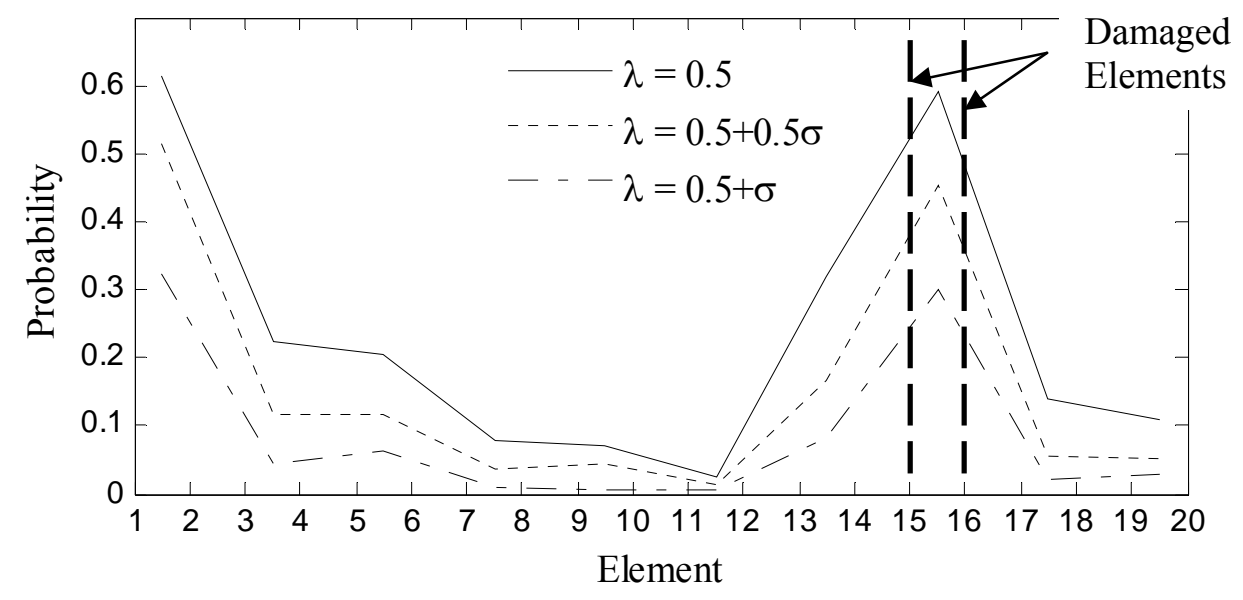

Figure 6-4. Probability of damage detection for the bridge column elements under 1\% measurement error

\subsubsection{Applying local NDT}

In the suspected damage area that is identified by the global NDT, the local NDT can be applied in order to detect the damage severity. In this numerical study, it is legitimate to 
assume SonReb with the regression models of Eq. (4.12) or Eq. (4.13) can provide an unbiased estimation for the compressive strength in the damaged element, $f_{c, d a m}$. However, the model errors are different in Eq. (4.12) or Eq. (4.13). Thus, one can assume that with Eq. (4.12) the estimate for $\sqrt{f_{c, d a m}}$ has a mean of $5.753 \mathrm{MPa}$ and a standard deviation of $0.5192 \mathrm{MPa}$, while the estimate for $\sqrt{f_{c, d a m}}$ using Eq. (4.13) has the same mean but the standard deviation of $0.8024 \mathrm{MPa}$.

\subsubsection{Assessing fragilities}

\subsubsection{Importance Measure}

To account for the inherent randomness of the structural properties in the fragility estimate for the damaged FEM, some quantities are considered as random variables and their statistic information is shown in Table 6-1. Note that the statistic information about the quantities $f_{c, d}, f_{c, c}, K_{a b u t, t}$ and $\sqrt{f_{c, d a m}}$ are obtained from NDT. As pointed out in the previous section, there are different ways to estimate fragility depending on the treatment of the uncertainties. Therefore, it is worthy to first look at the contributions of each random variable to the variability in the limited function as shown in Eq. (3.13). This can be done by comparing important measures of random variables (Der Kiureghian and Ke 1995). A vector of importance measures, $\gamma$, for a vector of random variables, $\mathbf{y}$, defined by

$$
\boldsymbol{\gamma}^{T}=\frac{\boldsymbol{\alpha}^{T} \mathbf{J}_{\mathbf{u}^{*}, \mathbf{y}^{*}} \mathbf{S D}^{\prime}}{\left\|\boldsymbol{\alpha}^{T} \mathbf{J}_{\mathbf{u}^{*}, \mathbf{y}^{*}} \mathbf{S D}^{\prime}\right\|}
$$


where $\mathbf{y}=\left\{\Theta, \mathbf{x}_{r}\right\}, \boldsymbol{\alpha}=$ a row vector of the negative normalized gradient of limit state function evaluated at the design point (the most likely failure point) in the standard normal space $\mathbf{u}$ transformed from the original space $\mathbf{y}, \mathbf{J}_{\mathbf{u}^{*}, \mathbf{y}^{*}}=$ the Jacobian of the probability transformation from the original space $\mathbf{y}$ to the standard normal space $\mathbf{u}$ at the design point, and $\mathbf{S D}^{\prime}=$ standard deviation matrix of equivalent normal variables $\mathbf{y}^{\prime}$ that can be calculated from $\mathbf{y}^{\prime}=\mathbf{y}^{*}+\mathbf{J}_{\mathbf{u}^{*}, \mathbf{y}^{*}}\left(\mathbf{u}-\mathbf{u}^{*}\right)$ at the design point. The entries in $\mathbf{S D}^{\prime}$ corresponds to the square root of the diagonal entries of the covariance matrix $\Sigma^{\prime}=\mathbf{J}_{\mathbf{u}^{*}, \mathbf{y}^{*}}\left(\mathbf{J}_{\mathbf{u}^{*}, \mathbf{y}^{*}}\right)^{T}$. The larger of the absolute value of importance measure is, the larger effect of that corresponding random variable has on the variability of the limit state function. In other words, one can ignore the uncertainties contributed from those random variables that have relative small importance measures.

As the demand models are conditioning on the earthquake intensity measures, $P S A$ and $P G V$, one can choose different levels of PSA and PGV to check whether the importance measures changes. Herein, three cases are considered: Case 1 with $P S A / g=0.5 \quad$ and $P G V \cdot T_{1} / H_{c}=0.5$, Case $2 \quad$ with $\quad P S A / g=1.0 \quad$ and $P G V \cdot T_{1} / H_{c}=1.5$, and Case 3 with $P S A / g=3.0$ and $P G V \cdot T_{1} / H_{c}=2.5$. Those values of $P S A$ and $P G V$ are chosen so that different level of failure probabilities are included. Table 6-6 lists the importance measures of the random variables for the deformation and shear fragility estimates. For the deformation failure mode, $\varepsilon_{C \delta}$ and $\varepsilon_{D \delta}$ are two most important variables, and $\theta_{\delta 0}$ and $\theta_{\delta 2}$ are the next important ones. The 
variability contributed from $\mathbf{x}_{r}$ can be ignored. For the case of shear failure mode, $\varepsilon_{C v}$, $\varepsilon_{D v}, \theta_{v 0}$, and $\theta_{v 2}$ are the most important variables, the variability from $f_{c, c}$ and $f_{c, d a m}$ should be considered as well. The above observations hold true for all three cases with different levels of earthquake intensity measures.

These observations found in Table 6-6 further tell us the importance of appropriately accounting for uncertainties in the probabilistic capacity and demand models, especially the model errors that are used to describe the error due to the inexact model form. Furthermore, when the importance measures for the parameters of $\mathbf{x}_{r}$ are compared, accurately obtaining $f_{c, c}$ and $f_{c, d a m}$ for the shear fragility is necessary. Thus, the efforts should be made to obtain accurate estimation of $f_{c, c}$ and $f_{c, d a m}$, such as to adopt reliable NDT techniques. 
Table 6-6. Importance measures for deformation and shear failure modes

\begin{tabular}{|c|c|c|c|c|c|c|c|c|}
\hline & \multicolumn{4}{|c|}{ deformation } & \multicolumn{4}{|c|}{ shear } \\
\hline & symbol & Case 1 & Case 2 & Case 3 & symbol & Case 1 & Case 2 & Case 3 \\
\hline \multirow{5}{*}{$\begin{array}{c}\text { model } \\
\text { parameters } \\
\text { in capacity } \\
\text { model }\end{array}$} & $\theta_{C, \delta 1}$ & -0.1522 & -0.1555 & -0.1582 & - & - & - & - \\
\hline & $\theta_{C, \delta 2}$ & -0.0689 & -0.0703 & -0.0716 & $\theta_{C, v 1}$ & -0.1754 & -0.1825 & -0.1879 \\
\hline & $\theta_{C, \delta 3}$ & -0.0635 & -0.0649 & -0.0661 & $\theta_{C, v 2}$ & -0.1578 & -0.1656 & -0.1724 \\
\hline & $\sigma_{C, \delta}$ & 0.0536 & -0.0010 & -0.0514 & $\sigma_{C, v}$ & 0.0298 & 0.0089 & -0.0177 \\
\hline & $\varepsilon_{C, \delta}$ & $-\mathbf{0 . 5 8 0 7}$ & $-\mathbf{0 . 5 9 2 7}$ & -0.6091 & $\varepsilon_{C, v}$ & -0.6591 & -0.6581 & -0.6526 \\
\hline \multirow{7}{*}{$\begin{array}{c}\text { model } \\
\text { parameters } \\
\text { in demand } \\
\text { model }\end{array}$} & $\theta_{D, \delta 0}$ & 0.2608 & 0.2665 & 0.2712 & $\theta_{D, v 0}$ & 0.2966 & 0.3085 & 0.3176 \\
\hline & $\theta_{D, \delta 1}$ & -0.0537 & -0.0490 & -0.0332 & $\theta_{D, v 1}$ & -0.0475 & -0.0446 & -0.0315 \\
\hline & $\theta_{D, \delta 2}$ & -0.4001 & -0.3446 & -0.2465 & $\theta_{D, v 2}$ & -0.4283 & -0.3755 & -0.2723 \\
\hline & $\theta_{D, \delta 3}$ & -0.0401 & 0.0240 & 0.0552 & $\theta_{D, v 3}$ & -0.0420 & 0.0256 & 0.0595 \\
\hline & $\theta_{D, \delta 4}$ & 0.0521 & 0.0533 & 0.0542 & $\theta_{D, v 4}$ & 0.0000 & 0.0000 & 0.0000 \\
\hline & $\theta_{D, \delta 5}$ & 0.0081 & 0.0689 & 0.1612 & $\theta_{D, v 5}$ & 0.0077 & 0.0672 & 0.1593 \\
\hline & $\varepsilon_{D, \delta}$ & 0.6242 & 0.6380 & 0.6493 & $\varepsilon_{D, v}$ & 0.3994 & 0.4155 & 0.4278 \\
\hline \multirow{10}{*}{$\begin{array}{l}\text { geometric } \\
\text { and } \\
\text { material } \\
\text { parameters }\end{array}$} & $L_{1}$ & -0.0026 & -0.0006 & 0.0016 & $L_{1}$ & -0.0065 & -0.0104 & -0.0180 \\
\hline & $H_{c}$ & -0.0048 & -0.0725 & -0.0088 & $H_{c}$ & -0.0146 & -0.0154 & -0.0180 \\
\hline & $f_{y}$ & -0.0450 & -0.0446 & -0.0507 & $f_{y}$ & 0.0346 & 0.0520 & 0.0849 \\
\hline & $f_{c, c}$ & -0.0260 & -0.0216 & -0.0111 & $f_{c, c}$ & -0.2110 & -0.2388 & -0.2863 \\
\hline & $w_{t}$ & 0.0164 & 0.0144 & 0.0096 & $w_{t}$ & 0.0018 & 0.0048 & 0.0108 \\
\hline & $f_{y h}$ & 0.0004 & 0.0001 & -0.0010 & $f_{y h}$ & -0.0701 & -0.0719 & -0.0716 \\
\hline & cover & 0.0066 & 0.0061 & 0.0063 & cover & -0.0020 & -0.0040 & -0.0081 \\
\hline & $\sqrt{f_{c, d a m}}$ & -0.0008 & -0.0003 & 0.0012 & $\sqrt{f_{c, d a m}}$ & 0.1521 & 0.1576 & 0.1603 \\
\hline & $f_{c, d}$ & -0.0001 & 0.0000 & 0.0001 & $f_{c, d}$ & 0.0007 & 0.0008 & 0.0002 \\
\hline & $K_{a b u t, t}$ & 0.0000 & 0.0000 & 0.0000 & $K_{a b u t, t}$ & -0.0001 & 0.0000 & -0.0001 \\
\hline
\end{tabular}

\subsubsection{Fragility estimates}

The marginal distribution of $\boldsymbol{\theta}$ in Eqs. (2.1) and (3.1) is multi-variate $t$ distribution, which can be approximately considered as Normal distribution since the number of data used for accessing $\boldsymbol{\theta}$ is large. With the variability in the structural properties being 
ignored, which has been verified by the importance analysis, the capacity (Eq. (2.1)) and demand (Eq. (3.1)) are multivariate normally distributed because they are linear combinations with the components that have univariate normal distributions. Thus, the fragility estimates for deformation and shear failure modes can be written as,

$$
\tilde{F}(\mathbf{s})=1-\Phi\left(\frac{\mu_{C, k}-\mu_{D, k}}{\sqrt{\tilde{\sigma}_{C, k}^{2}+\tilde{\sigma}_{D, k}^{2}}}\right) \quad k=\delta, v
$$

where $\Phi(\cdot)=$ cumulative distribution function (CDF) of a standard normal random variable, $\mu_{C, k}$ and $\mu_{D, k}=$ mean estimates of the capacity and demand models for the $k^{\text {th }}$ failure mode, and $\tilde{\sigma}_{C, k}$ and $\tilde{\sigma}_{D, k}=$ standard deviation estimates of the capacity and demand models for the $k^{\text {th }}$ failure mode. Since the capacity and demand are the linear functions of normal distribution random variables, $\tilde{\sigma}_{C, k}$ and $\tilde{\sigma}_{D, k}=$ can be calculated straightforwardly when considering the variability from $\boldsymbol{\theta}$ and the model errors $\sigma_{C, k} \varepsilon_{k}$ and $\sigma_{D, k} \varepsilon_{k}$. If the variability in $f_{c, c}$ and $f_{c, \text { dam }}$ needs to be accounted, the first order second moment approximation (FOSM) can be applied.

Similarly, the fragility for the deformation-shear bi-variate failure mode can be calculated by

$$
\tilde{F}(\mathbf{s})=1-\Phi_{2}\left(\frac{\mu_{C, \delta}-\mu_{D, \delta}}{\sqrt{\tilde{\sigma}_{C, \delta}^{2}+\tilde{\sigma}_{D, \delta}^{2}}}, \frac{\mu_{C, v}-\mu_{D, v}}{\sqrt{\tilde{\sigma}_{C, v}^{2}+\tilde{\sigma}_{D, v}^{2}}}, \rho_{\delta v}\right)
$$

where $\Phi_{2}(\cdot)$ is the bi-variety CDF of two standard normal random variables, and $\rho_{\delta v}=$ the correlation between $g_{\delta}$ and $g_{v}$. If the capacity and demand models are 
statistically independent, then $\rho_{\delta v}$ can be calculated by

$$
\rho_{\delta v}=\frac{\rho_{C, \delta v} \sigma_{C, \delta} \sigma_{C, v}+\rho_{D, \delta v} \sigma_{D, \delta} \sigma_{D, v}}{\sqrt{\left(\tilde{\sigma}_{C, \delta}^{2}+\tilde{\sigma}_{D, \delta}^{2}\right)\left(\tilde{\sigma}_{C, v}^{2}+\tilde{\sigma}_{D, v}^{2}\right)}}
$$

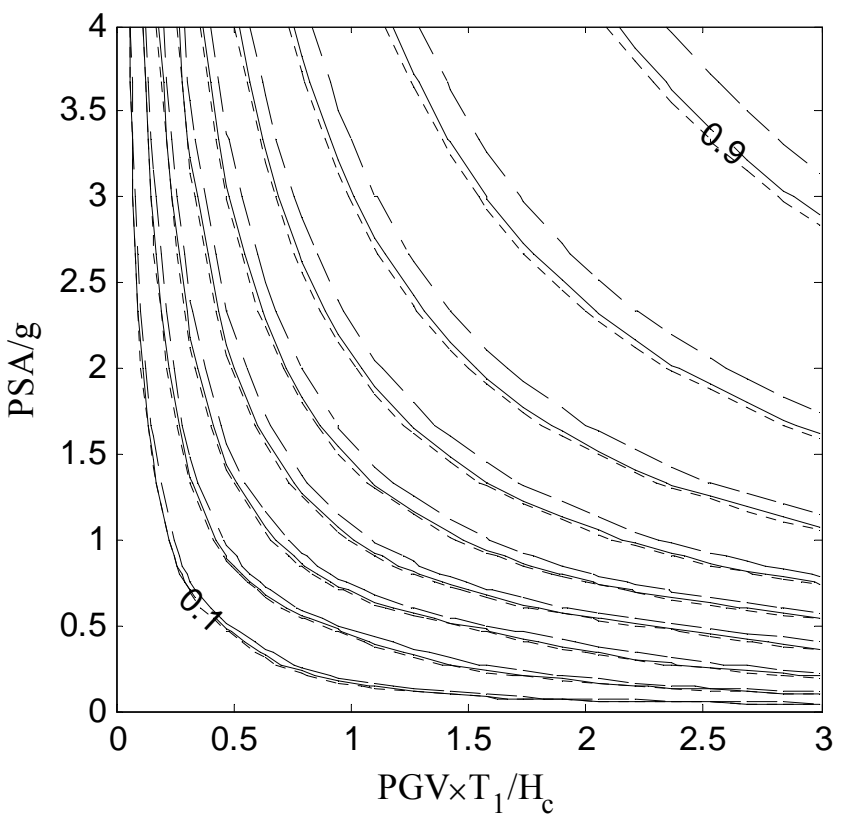

Figure 6-5. Deformation fragilities of the target damaged FEM (solid lines), the preliminary FEM (large dashed lines), and the identified damaged FEM (small dashed lines) 


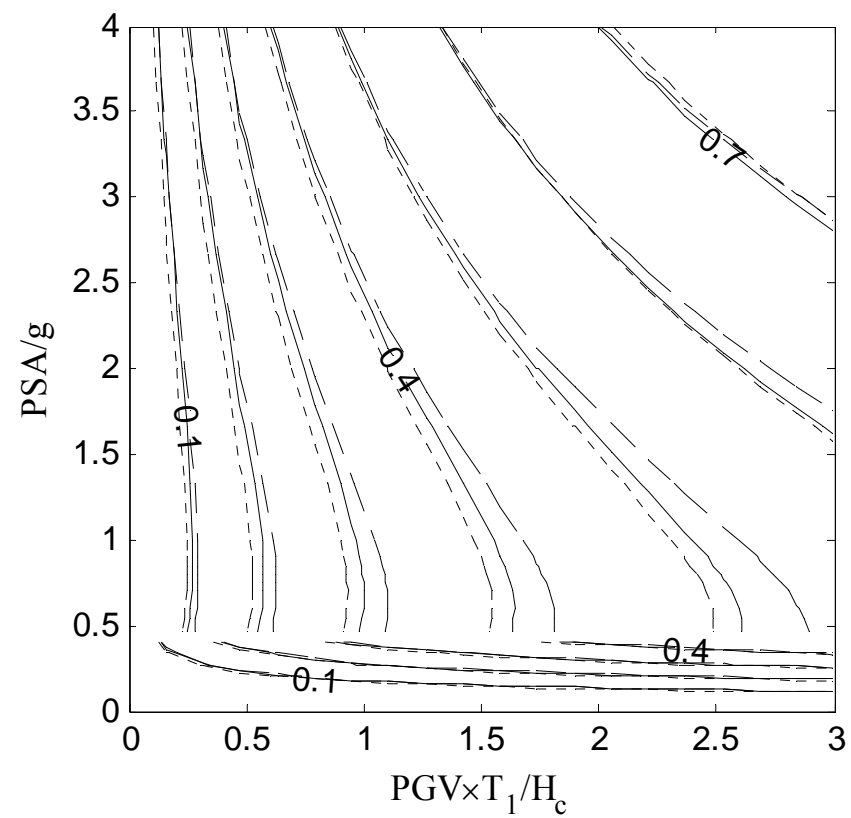

Figure 6-6. Shear fragility of the target damaged FEM (solid lines), the preliminary FEM (large dashed lines), and the identified damaged FEM (small dashed lines)

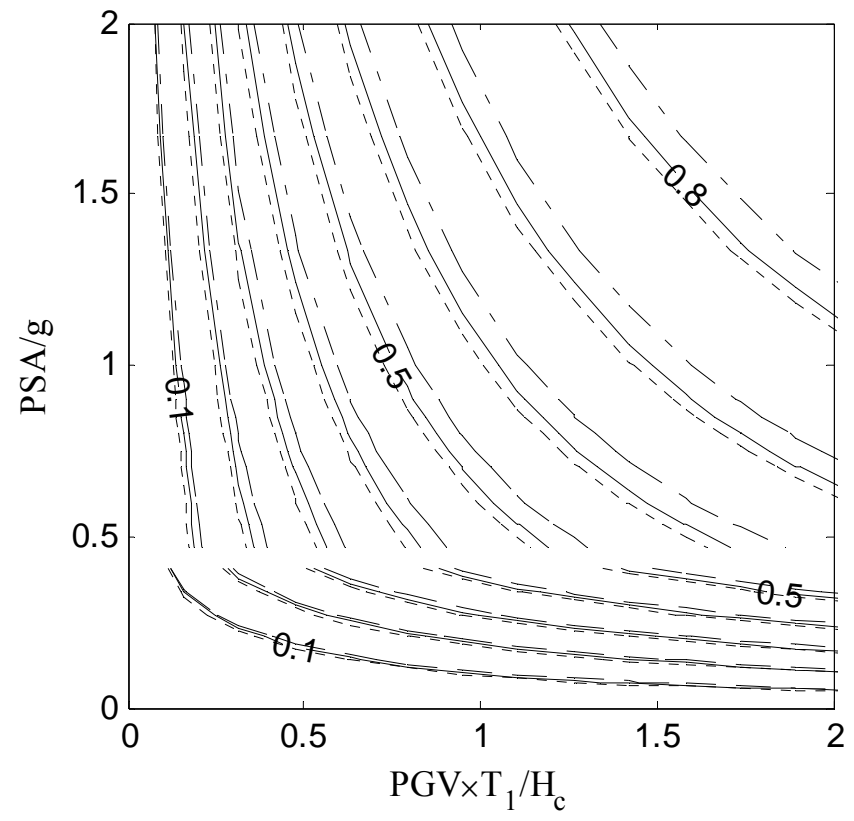

Figure 6-7. Bi-variate fragility of the target damaged FEM (solid lines), the preliminary FEM (large dashed lines), and the identified damaged FEM (small dashed lines) 
Figures 6-5 to 6-7 show the fragilities for deformation, shear, and bi-variate failure modes. Notice that the change in the variability of the shear demand models results in the discontinuities in fragility contour lines in shear and bi-variate fragilities because different weights are used for shear demand model assessment. Each contour represents a fragility level in the range of 0.1 to 0.9 for a given pair of normalized $P S A / g$ and $P G V \cdot T_{1} / H_{c}$. Note that in reality, the exact values of the structural properties of the target damaged FEM are unknown, thus the true fragility is unknown. However, in this numerical study, the properties of the target damaged FEM are known, therefore one can use the corresponding fragility to check the accuracy of the fragilities using the identified FEM.

In Figures 6-5 to 6-7, the solid line denotes the fragilities of the target damaged FEM, the dash-dotted line represents the fragility based on the preliminary values, and the dotted lines shows the fragility based on the identified FEM using the NDT methods. The difference between the solid lines and the dash-dotted lines are shown that using the preliminary values can not reflect the bridge performance correctly especially when the intensity measures become higher for the deformation and bi-variate fragilities where this difference becomes more obvious. On the other hand, the solid lines and the dotted lines are consistently close to each other indicating that the fragilities using the identified structural properties obtained from NDT are accurate enough to reflect the true performance of the bridge.

\subsection{Conclusions}

In this Section, the framework proposed by this dissertation is illustrated by a RC bridge 
with a single-column bent. A damage scenario is modeled by reducing compressive concrete strength of two elements in the column. The global NDT is applied first to identify the damage area and then a specific local NDT, SonReb (a combination of ultrasonic pulse velocity and rebound number tests) is used to assess the compressive strength of concrete in the suspected damage area. With the identified structural properties obtained from NDT, the probabilistic capacity and seismic demand are assessed. Accordingly, the fragilities are updated through reliability analysis.

To access the effects of each random variable on the variance of the limit state function, importance analysis is conducted. As the results of the importance measure, the variance from model parameters of the probabilistic capacity and demand models have much more impact on the fragility estimates than the variance brought in by the structural properties. Based on this finding, the fragility estimate can be assessed by a closed form, which saves much computation time by avoiding using any conventional reliability analysis. Additionally, the importance analysis shows that the concrete compressive strength of the column is important among the structural properties for the shear fragility estimate, and the properties of the superstructures are shown to be not significant. This is due to the fact that the failure is respect to the failure of the column. Furthermore, the result of the illustration shows that the proposed framework can successfully provide up-to-date structural properties and accurate fragility estimates. 


\section{CONCLUSIONS AND FUTURE WORK}

\subsection{Conclusions}

In this dissertation, an adaptive reliability analysis of RC bridges was developed based on the proposed probabilistic capacity models and demand models. The structural properties obtained from a combination of global and local NDT methods were used to capture the actual deterioration of bridges over time. The proposed framework accounts for the prevailing uncertainties in the capacity models and demand model, and the measurement and modeling errors in the NDT process. Avoiding the use of deterioration models, the proposed adaptive framework has three advantages:

- it takes into account all sources of information (sensed data, rules of mechanics, experimental data, and measurement errors), instead of using assumed values as conventional reliability analysis usually tends to do;

- it accounts for all causes of deterioration and is not limited to specific degrading mechanisms;

- the information obtained from damage detection using NDT can be used to predict future degradation and service life of an RC bridge by calibrating the existing damage models.

The proposed work not only provides accurate information about current health conditions of RC bridges, but also provides important information to deal with the problems of maintenance, repair and rehabilitation. Moreover, while the focus of the proposed work is on bridges, the same technology will be available for other civil 
engineering systems including locks, walls, buildings, pipelines, dams, tunnels, seaports, highways, railroads, and power generation stations. Additional advancements with respect to the current research are also proposed in terms of the interpretation and use of the data from NDT. The permanent monitoring of bridges will foster the creation of a database of the damage history of the system, which is not only important for accurate assessment of the reliability and better prediction of the service-life prediction of a system but also for developing optimal lifetime reliability-based maintenance strategies for bridges.

\section{2. $\quad$ Future Work}

My future research interests will continue in two interrelated directions: structural reliability and the application of NDT and structural health monitoring (SHM). In the near term, I plan to expand the framework developed in this dissertation to other types of structures such as different classes of RC bridges and steel bridges. I also plan to apply the developed framework to evaluate the safety of transportation networks using the upto-date structural properties of the individual bridges.

Although NDT and SHM techniques are developed to assess the in-situ properties of existing structures, an accurate deterioration model is still needed to predict future structure performance. I plan to better understand the deteriorating behavior of structural materials. My focus will be to incorporate the uncertainties in the material itself and in the factors that trigger the deterioration process. The application of stochastic process and spatial statistics will be used to pursue this area. I am also 
interested in developing probabilistic demand models for natural hazards, such as earthquakes, floods, and hurricanes, and man-made hazards like vehicle impact. In the model development, one needs to consider not only the uncertainties from the infrastructure itself but also the stochastic characteristics of the hazards. Modeling using discrete stochastic processes is suitable for this purpose.

Today, the development of NDT and SHM technologies for evaluating existing or newly built structures have now attained some degree of maturity. However, the implementations of these technologies and their applications have many challenges, which open many new research areas to explore. Particularly, I am interested in evaluating the reliability of different NDT methods and the accuracy of damage detection methods, considering the measurement and modeling uncertainties. Understanding where errors might occur and how the errors propagate will help us appropriately use the identified values of structural properties. I am also interested in performance-based SHM. For a given number of sensors, optimizing the sensor layout should be determined by minimizing the uncertainties in the identified model parameters and providing the most valuable information for the structural performance analysis. With the constraint of the available funding and the importance and sensitivity of the monitoring data used to evaluate structural performance, the optimal number and configuration of sensors can be obtained.

In addition to these specific interests, I am also interested in working more broadly on reliability of complex systems, structural behavior under stochastic excitations, risk assessment, and decision making under uncertainty. 


\section{REFERENCES}

Abramhamson, N. A., and Silva, W. J. (1997). "Empirical response spectral attenuation relations for shallow crustal earthquakes," Seismological Research Letters, 68(1), 94-127.

ACI Committee 318 (1995). "Building code requirements for structural concrete (ACI 318-95) and commentary (318R-95)," American Concrete Institute, Farmington Hills, Michigan.

ACI Committee 228 (2003). "In-place methods for determination of strength of concrete", Technical Committee Document 228.1R-03, American Concrete Institute, Detroit, Michigan.

Akaike, H. (1974). "A new look at the statistical model identification," IEEE Transaction on Automatic Control, 19(6), 716-723.

Akkar, S., and Metin, A. (2007). "Assessment of improved nonlinear static procedures in FEMA-440,” ASCE Journal of Structural Engineering, 133(9), 1237-1246.

Alsiwat, J. M., and Saatcioglu, M. (1992). "Reinforcement anchorage slip under monotonic loading," ASCE Journal of Structural Engineering, 118(9), 2421-2438.

Alvandi, A., and Cremona, C. (2006). "Assessment of vibration-based damage identification techniques", Journal of Sound and Vibration, 292, 179-202.

American Association of State Highway and Transportation Officials (2008). "Bridging the Gap: Restoring and rebuilding the nation's bridge," http://www.transportation1.org/BridgeReport/docs/BridgingtheGap.pdf

Applied Technology Council (ATC) (1996). "Seismic evaluation and retrofit of concrete buildings," Report No. ATC-40, Vols 1 and 2, Redwood City, California.

Applied Technology Council (ATC) (2005). "Improvement of nonlinear static seismic analysis procedures," Report No. FEMA-440, Redwood City, California.

Applied Technology Council (ATC) (1996). "Improved seismic design criteria for California bridges: Provisional recommendations," Report No. ATC-32, Redwood City, California.

Arici, Y., and Mosalam, K. M. (2005). "Statistical significance of modal parameters of bridge systems identified from strong motion data," Earthquake Engineering and Structural Dynamics, 34, 1323-1341. 
ASCE-ACI Joint Task Committee 426 (1973). "Shear strength of reinforced concrete members," ASCE Journal of Structural Engineering, 99(6), 1091-1187.

ASSHTO (1992). Standard specifications for highway bridges, $15^{\text {th }}$ ed., AASHTO, Washington, DC.

Baker, J. W., and Cornell, C. A. (2008). "Vector-valued measures for pulse-like nearfault ground motions," Engineering Structures, 30(4), 1048-1057.

Banan, M. R., Banan, M. R., and Hjelmstad, K. D. (1994a). "Parameter estimation of structures from static response: I. Computational aspects," ASCE Journal of Structural Engineering, 120(11), 3243-3255.

Banan, M. R., Banan, M. R., and Hjelmstad, K. D. (1994b). "Parameter estimation of structures from static response: II. Numerical simulation studies," ASCE Journal of Structural Engineering, 120(11), 3259-3283.

Barroso, L. R., and Rodriguez, R. (2004). "Damage detection utilizing the damage index method to a benchmark structure," ASCE Journal of Engineering Mechanics, 130(2), 142-151.

Beck, J. L., and Katafygiotis, L. S. (1998). "Updating models and their uncertainties. Part I: Bayesian statistical framework," ASCE Journal of Engineering Mechanics, 124(4), 455-461.

Bolton, R., Sikorsky, C., Park, S., Choi, S., and Stubbs, N. (2005). "Modal property changes of a seismically damaged concrete bridge," Journal of Bridge Engineering, $10(4), 415-428$.

Bolton, R., Stubbs, N., and Choi, S. (2001). "Documentation of change in modal properties of a concrete box-Girder bridge due to environmental and internal conditions," Computer-Aided Civil and Infrastructure Engineering, 16, 42-57.

Box, G. E. P., and Cox, D. R. (1964). "An analysis of transformations," Journal of the Royal Statistical Society, Series B 26, 211-246.

Box, G. E. P., and Tiao, G. C. (1992). Bayesian inference in statistical analysis, Wiley, New York.

Bray, J. D., and Rodriguez-Marek, A. (2004). "Characterization of forward-directivity ground motions in the near-fault region," Soil Dynamics and Earthquake Engineering, 24, 815-828.

Brincker, R., Ventura, C. E., and Andersen, P. (2001). "Damping estimation by 
frequency domain decomposition," Proceedings of the 19th International Modal Analysis Conference, Leuven, Belgium, 698-703.

Brownjohn, J. M. W., Moyo, P., Omenzetter, P., and Lu, Y. (2003). "Assessment of highway bridge upgrading by dynamic testing and finite-element model updating," Journal of Bridge Engineering, 8(3), 162-172.

Bu, J. Q., Law, S. S., and Zhu, X. Q. (2006). "Innovative bridge condition assessment from dynamic response of a passing vehicle," ASCE Journal of Engineering Mechanics, 132(12), 1372-1379.

Building Seismic Safety Council (BSSC) (1997). "NEHRP guidelines for the seismic rehabilitation of building," Report FEMA-273 (Guidelines) and Report FEMA-274 (Commentary), Washington, D.C.

Byers, W. G., Marley, M. J., Mohammadi, J., Nielsen, R. J., and Sarkani, S. (1997). "Fatigue reliability reassessment procedures: State-of-the-art paper," ASCE Journal of Structural Engineering, 123(3), 271-276.

Caltrans (2000). Seismic design criteria 1.4, California Department of Transportation (Caltrans).

Catbas, F. N., Brown, D. L., and Aktan, A. E. (2006). "Use of modal flexibility for damage detection and condition assessment: Case studies and demonstrations on large structures," ASCE Journal of Structural Engineering, 132(11), 1699-1712.

Chang, P. C., and Liu, S. C. (2003). "Recent research in nondestructive evaluation of civil infrastructures," Journal of Materials in Civil Engineering, 15(3), 298-304.

Chib, S., and Greenberg, E. (1995). "Understanding the Metropolis-Hastings algorithm," American Statistics, 49, 327-335.

Choe, D., Gardoni, P., and Rosowsky, D. (2007). "Closed-form fragility estimates parameter sensitivity and Bayesian updating for RC columns," ASCE Journal of Engineering Mechanics, 133(7), 833-843.

Choe, D., Gardoni, P., Rosowsky, D., and Haukaas, T. (2008). "Probabilistic Capacity Models and Seismic Fragility Estimates for RC Columns Subject to Corrosion," Reliability Engineering and System Safety, 93(3), 383-393.

Choi, S., Park, S., Bolton, R., Stubbs, N., and Sikorsky, C. (2004). "Periodic monitoring of physical property changes in a concrete box-girder bridge," Journal of Sound and Vibration, 274, 365-381. 
Cook, R. D., and Weisberg, S. (1997). "Graphics for assessing the adequacy of regression models," Journal of the American Statistical Association, 92, 490-499.

Cornwell, P., Doebling, S. W., and Farrar, C. R. (1997). "Application of the strain energy damage detection method to plate like structures," Proceedings of 15th International Modal Analysis Conference, Orlando, Florida, 1312-1318.

Cornell, C.A., and Krawinkler, H. (2000). "Progress and challenges in seismic performance assessment," PEER Center News, 3(2), $<$ http://peer.berkeley.edu/news/2000spring/index.html > (Jan. 19 2010).

Der Kiureghian, A., and Ke, J.-B. (1995). "Finite-element based reliability analysis of frame structures," The 4th International Conference on Structural Safety and Reliability, New York, 395-404.

Doebling, S. W., Farrar, C. R., and Prime, M. B. (1998). "A summary review of vibration-based damage identification methods," The Shock and Vibration Digest, 30(2), 91-105.

Doebling, S. W. and Farrar, C. R. (2001). "Estimation of statistical distribution for modal parameters identified from average Frequency response function data," Journal of Vibration and Control, 7, 603-624.

Efron, B. (1982). The jackknife, the bootstrap and other resampling plans, Society for industry and Applied Mathematics, Philadelphia, Pennsylvania.

Enright, M. P. and Frangopol, D. M. (1998). "Probabilistic analysis of resistance degradation of reinforced concrete bridge beams under corrosion," Engineering Structures, 20(11), 960-971.

Estes, A. C., and Frangopol, D. M. (2003). "Updating bridge reliability based on bridge management systems visual inspection results," Journal of Bridge Engineering, 8(6), 374-382.

Estes, A. C., Frangopol, D. M., and Foltz, S. D. (2003). "Updating the reliability of engineering structures using visual inspection results," Applications of statistics and probability in civil engineering, Vols 1 and 2, D. A. Der Kiureghian, S. Madanant, and J. M. Pestana, eds., Millpress, Rotterdam, 1087-1092.

European Committee for Standardization (CEN) (2001). "Eurocode 8: Design of structures for earthquake resistance," Part 1, European Standard prEN 1998-1, Draft No. 4, Brussels, Belgium. 
Farrar, C. R., Doebling, S. W., and Nix, D. A. (2001). "Vibration-based structural damage identification," Philosophical Transactions of the Royal Society of London Series a-Mathematical Physical and Engineering Sciences, 359(1778), 131-149.

Fajfar, P., and Gašperšič, P. (1996). "The N2 method for the seismic damage analysis of RC buildings," Earthquake Engineering and Structural Dynamics, 25, 31-46.

Fajfar, P. (2000). "A nonlinear analysis method for performance-based seismic design," Earthquake Spectra, 16(3), 573-592.

Feng, M. Q., Kim, D. K., and Yi, J.-H., and Chen, Y. (2004). "Baseline models for bridge performance monitoring," ASCE Journal of Engineering Mechanics, 130(5), 562-569.

Fox, C. H. J. (1992). "The location of defects in structures: a comparison of the use of natural frequency and mode shape data," Proceedings of the 10th International Modal Analysis Conference, 522-528.

Frangopol, D. M., Kong, J. S., and Gharaibeh, E. S. (2001). "Reliability-based life-Cycle management of highway bridges," Journal of Computing in Civil Engineering, 15(1), 27-34.

Friswell, M. I., and Mottershead, J. E. (1995). Finite element model updating in structural dynamics, The Netherlands: Kluwer Academic, Dordrecht.

Fritzen, C. P. (2005). "Vibration-based structural health monitoring - Concepts and applications," Key Engineering Materials, Damage Assessment of Structures VI, 293-294, 3-20.

Gardoni, P., Der Kiureghian, A., and Mosalam, K. M. (2002). "Probabilistic capacity models and fragility estimates for RC columns based on experimental observations," ASCE Journal of Engineering Mechanics, 128(10), 1024-1038.

Gardoni, P., Mosalam, K. M., and Der Kiureghian, A. (2003). "Probabilistic seismic demand models and fragility estimates for RC columns," Journal of Earthquake Engineering, 7(1), 79-106.

Gelman, A. G., Roberts, G. O., and Gilks, W. R. (1996). "Efficient metropolis jumping rules," Bayesian Statistics 5, J. M. Bernardo, J. Berger, A. P. Dawid, and A. F. M. Smith, eds., Oxford, Oxford University Press, 599-608.

Geweke, J. (1992). "Evaluating the accuracy of sampling-based approaches to the calculation of posterior moments," Bayesian statistics 4, J. M. Bernardo, J. Berger, A. P. Dawid, and A. F. M. Smith, eds., Oxford, Oxford University Press, 169-193. 
Gilks, W. R., Richardson, S., and Spiegelhalter, D. J. (1996). Markov chain monte carlo in practice, London, Chapman \& Hall.

Goel, R. K., and Chopra, A. K. (2004). "Evaluation of modal and FEMA pushover analysis: SAC buildings," Earthquake Spectra, 20(1), 225-254.

Goel, R. K. (2007). "Evaluation of current nonlinear static procedures using strong motion records," ASCE Structures Congress 2007, Long Beach, California.

Haario, H., Laine, M., Mira, A., and Saksman, E. (2006). "DRAM: Efficient adaptive MCMC," Statistics and Computing, 16(4), 339-354.

Haukaas, T., and Gardoni, P., (2009). "Model uncertainty in finite element analysis: Bayesian finite elements," ASCE Journal of Engineering Mechanics (Submitted).

Haukaas T., and Der Kiureghian, A. (2004). "Finite element reliability and sensitivity methods for performance-based earthquake engineering," Report No. PEER 2003/14, Pacific Earthquake Engineering Research Center, University of California, Berkeley.

Heintz, A. J., and Miranda, E. (2007). "Overview of FEMA 440 Recommendations and Improvement of Modeling of Nonlinear Degrading Response," ASCE Structures Congress 2007, Long Beach, California.

Hjelmstad, K. D., and Shin, S. (1997). "Damage detection and assessment of structures from static responses," ASCE Journal of Engineering Mechanics, 123(6), 568-576.

Humar, J., Bagchi, A., and Xu, H. (2006). "Performance of vibration-based techniques for the identification of structural damage," Structural Health Monitoring, 5(3), 215 227.

Hurlebaus, S., and Gaul, L. (2006). "Review: Smart structure dynamics," Mechanical Systems and Signal Processing, 20, 255-281.

Hurvich, C. M., and Tsai, C. (1989). "Regression and time series model selection in small samples,” Biometrika, 76(2), 297-307.

Huth, O., Feltrin, G., Maeck, J., Kilic, N., and Motavalli, M. (2005). "Damage identification using modal data: experiences on a prestressed concrete bridge," ASCE Journal of Structural Engineering, 131, 1898-1910.

Jaishi, B., and Ren, W. X. (2005). "Structural finite element model updating using ambient vibration test results," ASCE Journal of Structural Engineering, 131(4), 617-628. 
JMP (2007). Version 7, Statistical discovery software, Copyright (C) 2008 SAS Institute Inc., www.jmp.com.

Kalkan, E., and Kunnath, S. (2006). "Adaptive modal combination procedure for nonlinear static analysis of building structures," ASCE Journal of Structural Engineering, 132(11), 1721-1731.

Katkhuad, H., Martinez, R., and Haldar, A. (2005). "Health assessment at local level with unknown input excitation," ASCE Journal of Structural Engineering, 131(6), 956-965.

Kim, B. H., Stubbs, N., and Park, T. (2005). "A new method to extract modal parameters using output-only responses," Journal of Sound and Vibration, 282, 215-230.

Kim, J. H., and Mander, J. B. (2007). "Influence of transverse reinforcement on elastic shear stiffness of cracked concrete elements," Engineering Structures, 29(8), 17981807.

Kim, J.-T., and Stubbs, N. (2002). "Improved damage identification method based on modal information," Journal of Sound and Vibration, 252(2), 223-238.

Ko, J. M., Wong, C. W., and Lam, H. F. (1994). "Damage detection in steel framed structures by vibration measurement approach," Proceedings of 12th International Modal Analysis Conference, 280-286.

Koh, C.G., Hong, B., and Liaw, C.-Y. (2000). "Parameter identification of large structural systems in time domain," ASCE Journal of Structural Engineering, 126(8), 957-963.

Krawinkler, H., Medina, R., and Alavi, B. (2003). "Seismic drift and ductility demands and their dependence on ground motions," Engineering Structures, 25, 637-653.

Krawinkler, H., and Nassar, A. A. (1992). "Seismic design based on ductility and cumulative damage demands and capacity," Nonlinear seismic analysis and design of reinforced concrete buildings, P. Fajfar and H. Krawinkler, eds., Elsevier Applied Science, New York.

Kumar, R., Gardoni, P., and Sanchez-Silva, M. (2009). "Effect of cumulative seismic damage and corrosion on the life-cycle of reinforced concrete bridges," Earthquake Engineering and Structural Dynamics, 38(7), 887-905.

Kunnath, S., and Kalkan, E. (2004). "Evaluation of seismic deformation demands using nonlinear procedures in multistory steel and concrete moment frames," ISET Journal of Earthquake Technology, 41(1), 159-182. 
Liu, P.-L. (1995). "Identification and damage detection of trusses using modal data," ASCE Journal of Structural Engineering, 121(4), 599-608.

Longman, R. W., and Juang, J. N. (1987). "A variance based confidence criterion for ERA identified modal parameters," Proceedings of the AAS/AIAA Astrodynamics Specialist Conference, Kalispell, Montana, 581-600.

Luco, N. (2002) "Probabilistic seismic demand analysis, SMRF connection fractures, and near-source effects," Ph.D. Dissertation, Department of Civil and Environmental Engineering, Stanford University, California.

Mackie, K., and Stojadinović, B. (2002). "Bridge abutment model sensitivity for probabilistic seismic demand evaluation," Proceedings of Third National Seismic Conference and Workshop on Bridges and Highways, CD-ROM, Portland, Oregon, April 28-May 1, 2002.

Mackie, K., and Stojadinović, B. (2003). "Seismic demands for performance-based design of vridges," PEER report 2003/16, Pacific Engineering Earthquake Research Center, University of California, Berkeley.

Maroney, B., Kutter, B., Romstad, K., Chai, Y. H., and Vanderbilt, E. (1994). "Interpretation of large scale bridge abutment test results," Proceedings of 3rd Annual Seismic Research Workshop, California Department of Transportation, Sacramento, California.

McKay, M. D., Conover, W. J., and Beckman, R. J. (1979). "A comparison of three methods for selecting values of input variables in the analysis of output from a computer code," Technometrics, 22(2), 239-245.

McKenna, F., and Fenves, G.L. (2000). "An object-oriented software design for parallel structural analysis," Proceedings of the Advanced Technology in Structural Engineering, ASCE Structures Congress 2000, Washington, D.C.

McKenna, F., and Fenves, G. L. (2002), "The OpenSees command language primer," Department. of Civil and Environmental Engineering, University of California, Berkeley, California.

Metropolis, N., Rosenbluth, A. W., Rosenbluth, M. N., Teller, A. H., and Teller, E. (1953). "Equation of state calculations by fast computing machines," The Journal of Chemical Physics, 21(6), 1087-1092.

Mira, A. (2001). “On Metropolis-Hastings algorithms with delayed rejection," Metraon, LIX(3-4), 231-241. 
Moehle, J. P., Lynn, A. C., Elwood, K., and Sezen, H. (1999). "Gravity load collapse of reinforced concrete frames during earthquakes," Proceedings of 1st U.S. - Japan Workshop on Performance-Based Design Methodology for Reinforced Concrete Building Structures, Maui, Hawaii, 175-189.

Moehle, J. P., Elwood, K., and Sezen, H. (2000). "Shear failure and axial load collapse of existing reinforced concrete columns," Proceedings of 2nd U.S. - Japan Workshop on Performance-Based Design Methodology for Reinforced Concrete Building Structures, Sapporo, Japan, 241-255.

Moehle, J., and Deierlein, G. G. (2004). "A framework methodology for performancebased earthquake engineering," International Workshop on Performance-Based Design, Bled, Slovenia.

Mosteller, F., and Tukey, J. W. (1977). Data analysis and regression, Addison-Wesley, Reading, Massachusetts.

Nair, K. K., Kiremidjian, A. S., and Law, K. H. (2006). "Time series-based damage detection and localization algorithm with application to the ASCE benchmark structure," Journal of Sound and Vibration, 291, 349-68.

Pandey, A. K., Biswas, M., and Samman, M. M. (1991). "Damage detection from changes in curvature mode shapes," Journal of Sound Vibration, 145(2), 321-32.

Papadimitriou, C., Beck, J. L., and Katafygiotis, L. S. (2001). "Updating robust reliability using structural test data," Probabilistic Engineering Mechanics, 16, 103113.

Papadopoulos, L. and Garcia, E. (1998). "Structural damage identification: a probabilistic approach,” AIAA Journal, 36(11), 2137-2145.

Park, S., Stubbs, N., Bolton, R., and Choi, S. (2001). "Field verification of the damage index method in a concrete box-girder bridge via visual inspection," Computer-Aided Civil and Infrastructure Engineering, 16, 58-70.

PEER Strong Motion Database (2000). <http://peer.berkeley.edu/smcat> (Sept 16, 2008). (C) Copyright 2000, Regents of the University of California.

Peerters, B., and De Roeck, G. (2001). "Stochastic system identification for operational modal analysis: A Review," Journal of Dynamic System, Measurement, and Control, 123(4), 659-667. 
Peil, U. (2003). "Life-Cycle prolongation of civil-engineering structures via monitoring," Structural health monitoring, F.-K. Chang, ed., Technomic Publishing, Lancaster, Pennsylvania, 64-78.

Peil, U., and Mehdianpour, M. (1999). "Life cycle prediction via monitoring," Safety and Reliability, 1 \& 2, 551-556.

Peterson, L. D., Bullock, S. J., and Doebling, S. W. (1996). "The statistical sensitivity of experimental modal frequencies and damping ratio to measurement noise," Modal Analysis: The International Journal of Analytical and Experimental Modal Analysis, 11(1), 63-75.

Pothisiri, T., and Hjelmstad, K. D. (2003). "Structural damage detection and assessment from modal response," ASCE Journal of Engineering Mechanics, 129(2), 135-145.

Priestley, M. J. N., Seible, F., and Sozen, M. A. (1996). Seismic design and retrofit of bridges, Wiley, New York.

Pujol, S., Ramfrez, J.A., and Sozen, M.A. (1999). "Drift capacity of reinforced concrete columns subjected to cyclic shear reversals," Seismic Response of Concrete Bridges, ACI international, Farmington Hills, Michigan, 255-274.

Ramamoorthy, S. K., Gardoni, P., and Bracci, J. M. (2008). "Probabilistic demand models and fragility curves for reinforced concrete frames," ASCE Journal of Structural Engineering, 132(10), 1563-1572.

Rao, C.R., and Toutenburg, H. (1997). Linear models, least squares and alternatives, Springer, New York.

Reinert, J. M., and Apostolakis, G. E. (2006). "Including model uncertainty in riskinformed decision making," Annals of Nuclear Energy, 33, 354-369.

Robert, C. P., and Casella, G. (1999). Monte Carlo statistical methods, Springer-Verlag, New York.

Schwarz, G. (1978). "Estimating the dimension of a model," Annals of Statistics, 6(2), 461-464.

Sheather, S. J. (2008). A modern approach to regression with $R$, Springer-Verlag, New York.

Shome, N., and Cornell, C. A. (1999). "Probabilistic seismic demand analysis of nonlinear structures," Reliability of Marine Structures Report No. RMS-35, Department of Civil and Environmental Engineering, Stanford University, 
California.

Simpson, T. W., Lin, D. K.J ., and Chen, W. (2001). "Sampling strategies for computer experiments: design and analysis," International Journal of Reliability and Application, 2(3), 209-240.

Sohn, H., Law, K. H. (1997). "A Bayesian probabilistic approach for structure damage detection,” Earthquake Engineering and Structural Dynamics, 26, 1259-1281.

Stewart, M. G. and Rosowsky, D. V. (1998). "Structural safety and serviceability of concrete bridges subject to corrosion," Journal of Infrastructure System, 4(4), 146155.

Stubbs N. and Kim J-T. (1996). "Damage localization in structures without baseline modal parameters," AIAA Journal, 34(8), 1644-49.

Stubbs, N., Park, S., Bolton, R. W., Choi, S., and Silorsky, C. (1999). "Non-destructive estimate of the rate of change of structural degradation of the Lavic road overcrossing," Report No. FHWA/CA/ESC-99/13, California Department of Transportation, Sacramento, California.

Teran-Gilmore, A., and Bahena-Arredondo, N. (2008). "Cumulative ductility spectra for seismic design of ductile structures subjected to long duration motions: Concept and theoretical background," Journal of Earthquake Engineering, 12, 152-172.

Teughels, A., Maeck, J., and Roeck, G. D. (2002). "Damage assessment by FE model updating using damage functions," Computers and Structures, 80, 1869-1879.

Trifunac, M. D. and Brady, A. G. (1975) "A study on the duration of strong earthquake ground motion," Bulletin of the Seismological Society of America, 65(3), 581-626.

Val, D. V., Stewart, M. G., and Melchers, R. E. (1997). "Effect of reinforcement corrosion on reliability of highway bridges," Engineering Structures, 20(11), 10101019.

Vamvatsikos, D., and Cornell, C.A. (2002). "Incremental dynamic analysis," Earthquake Engineering and Structural Dynamics, 31(3), 491-514.

Vamvatsikos, D., and Cornell, C. A. (2005). "Direct estimation of the seismic demand and capacity of MDOF systems through Incremental Dynamic Analysis of an SDOF approximation," ASCE Journal of Structural Engineering, 131(4), 589-599.

van de Lindt, J. W., and Goh, G. (2004). "Earthquake duration effect on structural reliability," ASCE Journal of Structural Engineering, 130(5), 821-826. 
Van Laarhoven, P. J., and Arts, E. H. L. (1987). Simulated annealing: Theory and applications, Reidel Publishers, Amsterdam.

Vu, K. A. T., and Stewart, M. G. (2000). "Structural reliability of concrete bridges including improved chloride-induced corrosion models," Structural Safety, 22(4), 313-333.

Wang, D., and Haldar, A. (1994). "Element-level system identification with unknown input information," ASCE Journal of Engineering Mechanics, 120(1), 159-176.

Williams, M. S., and Sexsmith, R. G. (1997). "Seismic assessment of concrete bridges using inelastic damage analysis," Engineering Structures, 19(3), 208-216.

Wilson, J. C., and Tan, B. S. (1990). "Bridge abutments: formulation of simple model for earthquake response analysis," ASCE Journal of Engineering Mechanics, 116(8), 1828-1837.

Xia, Y., Hao, H., Brownjohn, J. M. W., and Xia, P.-Q. (2002). "Damage identification of structures with uncertain frequency and mode shape data," Earthquake Engineering and Structural Dynamics, 31, 1053-1066.

Yu, L., Law, S. S., Link, M., Zhang, L. M. (1999). "Damage detection in bolted joint structures using element contribution on modal strain energy," Proceedings of 2nd International Conference on Identification in Engineering System, Swansea, 516-26.

Zhang, J., and Makris, N. (2001). "Seismic response analysis of highway overcrossings including soil-structure Interaction," PEER Report No. 2001/02, Pacific Engineering Earthquake Research Center, University of California, Berkeley.

Zhang, R., and Mahadevan, S. (2000). "Model uncertainty and Bayesian updating in reliability-based inspection," Structural Safety, 22(2), 145-160.

Zhao, Z. W., Haldar, A., and Breen, F. L. (1994). "Fatigue-reliability updating through inspections of steel bridges," ASCE Journal of Structural Engineering, 120(5), 1624-1643.

Zheng, R. H., and Ellingwood, B. R. (1998a). "Role of non-destructive evaluation in time-dependent reliability analysis," Structural Safety, 20(4), 325-339.

Zheng, R. H., and Ellingwood, B. R. (1998b). "Stochastic fatigue crack growth in steel structures subject to random loading." Structural Safety, 20(4), 303-323.

Zhong, J., Gardoni, P., Rosowsky, D., and Haukaas, T. (2008). "Probabilistic seismic demand models and fragility estimates for reinforced concrete bridges with two column bents," ASCE Journal of Engineering Mechanics, 134(6), 495-504. 


\section{APPENDIX A}

Table A.1. Parameters in the deformation capacity model

\begin{tabular}{cccccccc}
\hline \multirow{2}{*}{ Parameter } & \multirow{2}{*}{ Mean } & Standard & \multicolumn{5}{c}{ Correlation Coefficient } \\
\cline { 5 - 7 } & & Deviation & $\theta_{C, \delta 1}$ & $\theta_{C, \delta 2}$ & $\theta_{C, \delta 3}$ & $\sigma_{C, \delta}$ \\
\hline$\theta_{C, \delta 1}$ & 0.675 & 0.105 & 1.0 & & & \\
$\theta_{C, \delta 2}$ & 0.631 & 0.133 & -0.27 & 1.0 & & \\
$\theta_{C, \delta 3}$ & -57.5 & 10.1 & -0.63 & -0.39 & 1.0 & \\
$\sigma_{C, \delta}$ & 0.4 & 0.045 & -0.39 & -0.06 & -0.13 & 1.0 \\
\hline
\end{tabular}

Table A.2. Parameters in the shear capacity model

\begin{tabular}{ccccccc}
\hline \multirow{2}{*}{ Parameter } & Mean & $\begin{array}{c}\text { Standard } \\
\text { Deviation }\end{array}$ & \multicolumn{4}{c}{ Correlation Coefficient } \\
\cline { 5 - 7 } & & $\theta_{C, v 1}$ & $\theta_{C, v 2}$ & $\sigma_{C, v}$ \\
\hline$\theta_{C, v 1}$ & 18.3 & 1.45 & 1.0 & & \\
$\theta_{C, v 2}$ & -0.47 & 0.078 & -0.87 & 1.0 & \\
$\sigma_{C, v}$ & 0.185 & 0.018 & -0.04 & -0.02 & 1.0 \\
\hline
\end{tabular}

Table A.3. Parameters in the bivariate deformation-shear capacity model

\begin{tabular}{ccccccccccc}
\hline \multirow{2}{*}{ Parameter } & \multirow{2}{*}{ Mean } & Standard & \multicolumn{10}{c}{ Correlation Coefficient } \\
\cline { 4 - 11 } & & Deviation & $\theta_{C, \delta 1}$ & $\theta_{C, \delta 2}$ & $\theta_{C, \delta 3}$ & $\sigma_{C, \delta}$ & $\theta_{C, v 1}$ & $\theta_{C, v 2}$ & $\sigma_{C, v}$ & $\rho_{C}$ \\
\hline$\theta_{C, \delta 1}$ & 0.597 & 0.116 & 1.00 & & & & & & & \\
$\theta_{C, \delta 2}$ & 0.787 & 0.179 & -0.40 & 1.00 & & & & & & \\
$\theta_{C, \delta 3}$ & -56.44 & 11.071 & -0.60 & -0.34 & 1.00 & & & & & \\
$\sigma_{C, \delta}$ & 0.415 & 0.048 & -0.20 & 0.17 & 0.09 & 1.00 & & & & \\
$\theta_{C, v 1}$ & 17.039 & 1.519 & 0.00 & -0.02 & 0.04 & -0.05 & 1.00 & & & \\
$\theta_{C, v 2}$ & -0.446 & 0.081 & 0.06 & -0.14 & 0.03 & 0.05 & -0.84 & 1.00 & & \\
$\sigma_{C, v}$ & 0.196 & 0.019 & 0.09 & 0.03 & -0.11 & 0.15 & -0.12 & 0.12 & 1.00 & \\
$\rho_{C}$ & -0.463 & 0.152 & 0.15 & -0.22 & 0.03 & -0.09 & 0.13 & 0.00 & -0.10 & 1.00 \\
\hline
\end{tabular}




\section{APPENDIX B}

Table B. 1. The design parameters for the 60 bridge configurations

\begin{tabular}{|c|c|c|c|c|c|c|c|c|c|c|c|c|}
\hline \multirow{2}{*}{ Scenario } & \multicolumn{12}{|c|}{ Design Parameter } \\
\hline & $\alpha$ & $L_{1}$ & $H_{c}$ & $D_{c} / D_{s}$ & $f_{y}$ & $f_{c}^{\prime}$ & $\rho_{l}$ & $\rho_{s}$ & $w_{t}$ & $K_{\text {soil }}$ & $K_{a b u t}$ & $L_{2} / L_{1}$ \\
\hline 1 & 13.220 & 64.068 & 21.759 & 0.916 & 40.000 & 6.136 & 0.013 & 0.004 & 0.552 & $\mathrm{D}$ & $\mathrm{F}$ & 1.085 \\
\hline 2 & 46.780 & 76.271 & 17.661 & 1.062 & 77.288 & 4.695 & 0.022 & 0.010 & 0.243 & $\mathrm{~A}$ & $\mathrm{C}$ & 1.280 \\
\hline 3 & 29.492 & 96.610 & 27.749 & 0.670 & 93.136 & 5.881 & 0.023 & 0.010 & 0.607 & $\mathrm{D}$ & $\mathrm{D}$ & 1.364 \\
\hline 4 & 55.932 & 112.881 & 24.597 & 1.296 & 49.322 & 4.610 & 0.024 & 0.005 & 0.508 & $\mathrm{D}$ & $\mathrm{A}$ & 1.153 \\
\hline 5 & 27.458 & 175.932 & 23.651 & 0.759 & 58.644 & 5.458 & 0.038 & 0.011 & 0.287 & $\mathrm{~A}$ & $\mathrm{~F}$ & 1.432 \\
\hline 6 & 36.610 & 155.593 & 28.380 & 0.704 & 53.983 & 6.729 & 0.036 & 0.008 & 0.706 & $\mathrm{C}$ & $\mathrm{B}$ & 1.051 \\
\hline 7 & 35.593 & 80.339 & 28.064 & 0.983 & 71.695 & 4.780 & 0.023 & 0.004 & 0.728 & $\mathrm{C}$ & $\mathrm{F}$ & 1.390 \\
\hline 8 & 54.915 & 149.492 & 28.695 & 1.229 & 85.678 & 6.390 & 0.018 & 0.008 & 0.320 & $\mathrm{~A}$ & $\mathrm{D}$ & 1.008 \\
\hline 9 & 26.441 & 141.356 & 24.281 & 0.927 & 43.729 & 3.169 & 0.015 & 0.007 & 0.210 & B & $\mathrm{A}$ & 1.322 \\
\hline 10 & 4.068 & 88.475 & 32.163 & 0.938 & 89.407 & 3.847 & 0.027 & 0.009 & 0.122 & $\mathrm{~A}$ & $\mathrm{~F}$ & 1.042 \\
\hline 11 & 50.847 & 66.102 & 27.119 & 1.185 & 92.203 & 3.254 & 0.031 & 0.007 & 0.386 & $\mathrm{D}$ & $\mathrm{C}$ & 1.314 \\
\hline 12 & 45.763 & 145.424 & 20.814 & 1.173 & 54.915 & 4.949 & 0.012 & 0.009 & 0.695 & $\mathrm{~B}$ & $\mathrm{~A}$ & 1.246 \\
\hline 13 & 56.949 & 131.186 & 30.586 & 0.849 & 79.153 & 3.000 & 0.019 & 0.008 & 0.298 & $\mathrm{~A}$ & $\mathrm{~F}$ & 1.458 \\
\hline 14 & 22.373 & 125.085 & 16.715 & 1.308 & 62.373 & 6.814 & 0.018 & 0.008 & 0.419 & $\mathrm{~B}$ & $E$ & 1.034 \\
\hline 15 & 23.390 & 173.898 & 20.183 & 0.894 & 78.220 & 3.085 & 0.024 & 0.011 & 0.397 & $\mathrm{~B}$ & $E$ & 1.017 \\
\hline 16 & 33.559 & 167.797 & 30.902 & 1.073 & 91.271 & 3.678 & 0.033 & 0.007 & 0.265 & $\mathrm{~B}$ & $\mathrm{~A}$ & 1.127 \\
\hline 17 & 11.186 & 118.983 & 27.434 & 1.117 & 82.881 & 3.508 & 0.011 & 0.006 & 0.673 & $\mathrm{~A}$ & $\mathrm{D}$ & 1.212 \\
\hline 18 & 39.661 & 137.288 & 34.369 & 1.330 & 60.508 & 5.966 & 0.021 & 0.004 & 0.486 & $\mathrm{~A}$ & $\mathrm{~B}$ & 1.373 \\
\hline 19 & 34.576 & 60.000 & 35.000 & 1.285 & 67.034 & 5.034 & 0.017 & 0.009 & 0.464 & $\mathrm{~B}$ & $\mathrm{D}$ & 1.178 \\
\hline 20 & 3.051 & 159.661 & 33.424 & 0.681 & 61.441 & 5.627 & 0.028 & 0.007 & 0.453 & $\mathrm{D}$ & $E$ & 1.475 \\
\hline 21 & 10.169 & 94.576 & 23.336 & 0.815 & 52.119 & 4.271 & 0.030 & 0.006 & 0.596 & $\mathrm{~A}$ & B & 1.000 \\
\hline 22 & 19.322 & 100.678 & 31.217 & 0.860 & 45.593 & 5.119 & 0.030 & 0.009 & 0.739 & $\mathrm{~A}$ & $\mathrm{D}$ & 1.449 \\
\hline 23 & 9.153 & 92.542 & 24.912 & 1.274 & 64.237 & 7.576 & 0.019 & 0.010 & 0.530 & $\mathrm{C}$ & $\mathrm{A}$ & 1.466 \\
\hline
\end{tabular}


Table B. 1. (cont.) The design parameters for the 60 bridge configurations

\begin{tabular}{|c|c|c|c|c|c|c|c|c|c|c|c|c|}
\hline \multirow{2}{*}{ Scenario } & \multicolumn{12}{|c|}{ Design Parameter } \\
\hline & $\alpha$ & $L_{1}$ & $H_{c}$ & $D_{c} / D_{s}$ & $f_{y}$ & $f_{c}^{\prime}$ & $\rho_{l}$ & $\rho_{s}$ & $w_{t}$ & $K_{\text {soil }}$ & $K_{a b u t}$ & $L_{2} / L_{1}$ \\
\hline 24 & 0.000 & 116.949 & 23.966 & 1.263 & 80.085 & 5.288 & 0.012 & 0.005 & 0.232 & $\mathrm{D}$ & $\mathrm{D}$ & 1.424 \\
\hline 25 & 6.102 & 72.203 & 19.868 & 1.017 & 81.017 & 5.542 & 0.032 & 0.008 & 0.651 & B & G & 1.356 \\
\hline 26 & 2.034 & 147.458 & 26.803 & 1.050 & 65.169 & 4.864 & 0.038 & 0.005 & 0.254 & $\mathrm{D}$ & $\mathrm{A}$ & 1.254 \\
\hline 27 & 53.898 & 121.017 & 33.739 & 1.129 & 94.068 & 6.475 & 0.031 & 0.010 & 0.640 & $\mathrm{~A}$ & $\mathrm{~B}$ & 1.415 \\
\hline 28 & 21.356 & 110.847 & 30.271 & 0.827 & 53.051 & 7.661 & 0.010 & 0.010 & 0.684 & $\mathrm{C}$ & $\mathrm{C}$ & 1.161 \\
\hline 30 & 47.797 & 82.373 & 25.542 & 0.737 & 68.898 & 6.305 & 0.035 & 0.008 & 0.100 & $\mathrm{~B}$ & $\mathrm{~F}$ & 1.093 \\
\hline 31 & 37.627 & 163.729 & 17.346 & 1.106 & 69.831 & 7.153 & 0.040 & 0.010 & 0.188 & $\mathrm{C}$ & $\mathrm{B}$ & 1.229 \\
\hline 32 & 58.983 & 108.814 & 22.390 & 1.084 & 70.763 & 4.017 & 0.039 & 0.009 & 0.717 & $\mathrm{~B}$ & $\mathrm{~F}$ & 1.059 \\
\hline 33 & 38.644 & 70.169 & 18.607 & 0.994 & 47.458 & 8.000 & 0.037 & 0.006 & 0.353 & $\mathrm{C}$ & B & 1.136 \\
\hline 34 & 7.119 & 98.644 & 22.075 & 0.782 & 48.390 & 3.932 & 0.036 & 0.011 & 0.431 & $\mathrm{C}$ & $\mathrm{C}$ & 1.220 \\
\hline 35 & 52.881 & 151.525 & 29.641 & 1.140 & 50.254 & 3.763 & 0.039 & 0.009 & 0.408 & $\mathrm{C}$ & $E$ & 1.483 \\
\hline 36 & 24.407 & 114.915 & 26.488 & 1.095 & 57.712 & 7.746 & 0.035 & 0.010 & 0.144 & $\mathrm{C}$ & $\mathrm{G}$ & 1.347 \\
\hline 37 & 32.542 & 123.051 & 22.705 & 0.793 & 67.966 & 3.424 & 0.034 & 0.005 & 0.309 & $\mathrm{D}$ & $\mathrm{G}$ & 1.237 \\
\hline 38 & 1.017 & 74.237 & 26.173 & 0.692 & 90.339 & 5.797 & 0.016 & 0.006 & 0.111 & $\mathrm{~B}$ & $\mathrm{~A}$ & 1.186 \\
\hline 39 & 40.678 & 153.559 & 20.498 & 1.207 & 55.847 & 6.559 & 0.029 & 0.004 & 0.166 & $\mathrm{C}$ & $E$ & 1.331 \\
\hline 40 & 31.525 & 84.407 & 17.031 & 0.715 & 73.559 & 7.237 & 0.017 & 0.008 & 0.574 & A & $\mathrm{G}$ & 1.144 \\
\hline 41 & 12.203 & 102.712 & 31.532 & 0.972 & 40.932 & 7.831 & 0.020 & 0.007 & 0.177 & $\mathrm{~B}$ & $\mathrm{~B}$ & 1.305 \\
\hline 42 & 41.695 & 106.780 & 18.292 & 1.039 & 83.814 & 6.644 & 0.029 & 0.011 & 0.629 & $\mathrm{D}$ & A & 1.169 \\
\hline 43 & 5.085 & 86.441 & 25.858 & 1.319 & 41.864 & 5.373 & 0.026 & 0.007 & 0.442 & $\mathrm{~B}$ & $\mathrm{G}$ & 1.339 \\
\hline 44 & 44.746 & 180.000 & 34.685 & 0.804 & 87.542 & 6.051 & 0.021 & 0.004 & 0.133 & $\mathrm{D}$ & $\mathrm{C}$ & 1.102 \\
\hline 45 & 15.254 & 104.746 & 33.108 & 1.028 & 59.576 & 3.339 & 0.014 & 0.009 & 0.276 & $\mathrm{D}$ & $\mathrm{G}$ & 1.076 \\
\hline 46 & 8.136 & 127.119 & 31.847 & 1.218 & 88.475 & 7.492 & 0.033 & 0.006 & 0.375 & $\mathrm{~A}$ & $\mathrm{G}$ & 1.271 \\
\hline 47 & 20.339 & 161.695 & 19.553 & 1.252 & 63.305 & 3.593 & 0.025 & 0.005 & 0.618 & $\mathrm{C}$ & A & 1.441 \\
\hline 48 & 51.864 & 135.254 & 18.922 & 0.950 & 86.610 & 6.898 & 0.028 & 0.005 & 0.563 & $\mathrm{~A}$ & $\mathrm{C}$ & 1.288 \\
\hline 49 & 18.305 & 143.390 & 21.129 & 0.838 & 46.525 & 5.712 & 0.020 & 0.005 & 0.364 & $\mathrm{~A}$ & $\mathrm{G}$ & 1.381 \\
\hline
\end{tabular}


Table B. 1. (cont.) The design parameters for the 60 bridge configurations

\begin{tabular}{|c|c|c|c|c|c|c|c|c|c|c|c|c|}
\hline \multirow{2}{*}{ Scenario } & \multicolumn{12}{|c|}{ Design Parameter } \\
\hline & $\alpha$ & $L_{1}$ & $H_{c}$ & $D_{c} / D_{s}$ & $f_{y}$ & $f_{c}^{\prime}$ & $\rho_{l}$ & $\rho_{s}$ & $w_{t}$ & $K_{\text {soil }}$ & $K_{a b u t}$ & $L_{2} / L_{1}$ \\
\hline 50 & 25.424 & 171.864 & 29.956 & 1.162 & 81.949 & 4.525 & 0.011 & 0.011 & 0.342 & $\mathrm{D}$ & D & 1.398 \\
\hline 51 & 14.237 & 129.153 & 32.793 & 1.241 & 75.424 & 6.220 & 0.034 & 0.009 & 0.541 & $\mathrm{C}$ & $\mathrm{B}$ & 1.025 \\
\hline 52 & 60.000 & 68.136 & 29.325 & 0.905 & 42.797 & 5.203 & 0.025 & 0.009 & 0.497 & $\mathrm{D}$ & $\mathrm{E}$ & 1.203 \\
\hline 53 & 42.712 & 165.763 & 23.020 & 0.961 & 51.186 & 7.322 & 0.026 & 0.010 & 0.585 & $\mathrm{~B}$ & $\mathrm{G}$ & 1.110 \\
\hline 54 & 57.966 & 90.508 & 34.054 & 0.726 & 74.492 & 4.356 & 0.022 & 0.006 & 0.475 & $\mathrm{~B}$ & $\mathrm{C}$ & 1.068 \\
\hline 55 & 49.831 & 62.034 & 25.227 & 1.006 & 76.356 & 7.915 & 0.014 & 0.005 & 0.221 & $\mathrm{C}$ & $\mathrm{C}$ & 1.297 \\
\hline 56 & 48.814 & 139.322 & 17.976 & 0.771 & 56.780 & 6.983 & 0.016 & 0.008 & 0.331 & $\mathrm{C}$ & $E$ & 1.492 \\
\hline 57 & 43.729 & 157.627 & 16.400 & 0.748 & 84.746 & 4.102 & 0.013 & 0.006 & 0.155 & $\mathrm{~B}$ & $\mathrm{D}$ & 1.195 \\
\hline 58 & 30.508 & 133.220 & 21.444 & 1.196 & 95.000 & 7.068 & 0.015 & 0.007 & 0.750 & $\mathrm{D}$ & $\mathrm{F}$ & 1.500 \\
\hline 59 & 17.288 & 78.305 & 32.478 & 0.883 & 66.102 & 4.441 & 0.037 & 0.006 & 0.199 & A & $\mathrm{C}$ & 1.407 \\
\hline 60 & 16.271 & 177.966 & 19.237 & 0.871 & 72.627 & 7.407 & 0.032 & 0.007 & 0.519 & $\mathrm{D}$ & $\mathrm{E}$ & 1.263 \\
\hline
\end{tabular}




\section{APPENDIX C}

Table C. 1. Ground motion records for SMSR in Group 1

\begin{tabular}{|c|l|c|c|c|c|}
\hline Record ID & \multicolumn{1}{|c|}{ Event and Time } & M & R & GM & USGS \\
\hline P0552 & Chalfant Valley 1986/07/21 14:42 & 6.2 & 23 & A & - \\
\hline P0359 & Coalinga 1983/05/02 23:42 & 6.4 & 29.6 & A & - \\
\hline P0370 & Coalinga 1983/05/02 23:42 & 6.4 & 27.7 & A & - \\
\hline P0165 & Imperial Valley 1979/10/15 23:16 & 6.5 & 26.5 & A & B \\
\hline P0191 & Imperial Valley 1979/10/15 23:16 & 6.5 & 26 & A & B \\
\hline P0448 & Morgan Hill 1984/04/24 21:15 & 6.2 & 16.2 & A & A \\
\hline P0538 & N. Palm Springs 1986/07/08 09:20 & 6 & 25.8 & A & A \\
\hline P0612 & Whittier Narrows 1987/10/01 14:42 & 6 & 26.8 & A & - \\
\hline P0341 & Coalinga 1983/05/02 23:42 & 6.4 & 29.6 & B & - \\
\hline P0344 & Coalinga 1983/05/02 23:42 & 6.4 & 28.4 & B & - \\
\hline P0347 & Coalinga 1983/05/02 23:42 & 6.4 & 29.9 & B & - \\
\hline P0213 & Livermore 1980/01/24 19:00 & 5.8 & 29.8 & B & B \\
\hline P0215 & Livermore 1980/01/24 19:00 & 5.8 & 21.7 & B & C \\
\hline P0216 & Livermore 1980/01/24 19:00 & 5.8 & 17.6 & B & C \\
\hline P0462 & Morgan Hill 1984/04/24 21:15 & 6.2 & 22.7 & B & B \\
\hline P0464 & Morgan Hill 1984/04/24 21:15 & 6.2 & 16.2 & B & B \\
\hline P0606 & Whittier Narrows 1987/10/01 14:42 & 6 & 23.3 & B & - \\
\hline P0631 & Whittier Narrows 1987/10/01 14:42 & 6 & 27 & B & B \\
\hline P0639 & Whittier Narrows 1987/10/01 14:42 & 6 & 28.5 & B & C \\
\hline P0697 & Whittier Narrows 1987/10/01 14:42 & 6 & 29.3 & B & B \\
\hline
\end{tabular}


Table C. 2. Ground motion records for SMLR in Group 1

\begin{tabular}{|c|l|c|c|c|c|}
\hline \multirow{2}{*}{ Record ID } & \multicolumn{1}{|c|}{ Event and Time } & $\mathrm{M}$ & $\mathrm{R}$ & $\mathrm{GM}$ & USGS \\
\hline P0550 & Chalfant Valley 1986/07/21 14:42 & 6.2 & 33.4 & $\mathrm{~A}$ & - \\
\hline P0559 & Chalfant Valley 1986/07/21 14:42 & 6.2 & 40.6 & $\mathrm{~A}$ & - \\
\hline P0327 & Coalinga 1983/05/02 23:42 & 6.4 & 38.4 & $\mathrm{~A}$ & - \\
\hline P0357 & Coalinga 1983/05/02 23:42 & 6.4 & 34.4 & $\mathrm{~A}$ & - \\
\hline P0358 & Coalinga 1983/05/02 23:42 & 6.4 & 31.8 & $\mathrm{~A}$ & - \\
\hline P0364 & Coalinga 1983/05/02 23:42 & 6.4 & 32.3 & $\mathrm{~A}$ & - \\
\hline P0214 & Livermore 1980/01/24 19:00 & 5.8 & 31 & $\mathrm{~A}$ & $\mathrm{~B}$ \\
\hline P0477 & Morgan Hill 1984/04/24 21:15 & 6.2 & 44.1 & $\mathrm{~A}$ & $\mathrm{E}$ \\
\hline P0537 & N. Palm Springs 1986/07/08 09:20 & 6 & 43.8 & $\mathrm{~A}$ & - \\
\hline P0554 & Chalfant Valley 1986/07/21 14:42 & 6.2 & 36 & $\mathrm{~B}$ & - \\
\hline P0325 & Coalinga 1983/05/02 23:42 & 6.4 & 40.5 & $\mathrm{~B}$ & - \\
\hline P0353 & Coalinga 1983/05/02 23:42 & 6.4 & 38.8 & $\mathrm{~B}$ & - \\
\hline P0354 & Coalinga 1983/05/02 23:42 & 6.4 & 41 & $\mathrm{~B}$ & - \\
\hline P0355 & Coalinga 1983/05/02 23:42 & 6.4 & 47 & $\mathrm{~B}$ & - \\
\hline P0365 & Coalinga 1983/05/02 23:42 & 6.4 & 34.6 & $\mathrm{~B}$ & - \\
\hline P0456 & Morgan Hill 1984/04/24 21:15 & 6.2 & 31.4 & $\mathrm{~B}$ & $\mathrm{~B}$ \\
\hline P0518 & N. Palm Springs 1986/07/08 09:20 & 6 & 35.3 & $\mathrm{~B}$ & $\mathrm{~B}$ \\
\hline P0522 & N. Palm Springs 1986/07/08 09:20 & 6 & 34.9 & $\mathrm{~B}$ & $\mathrm{~B}$ \\
\hline P0694 & Whittier Narrows 1987/10/01 14:42 & 6 & 32.6 & $\mathrm{~B}$ & $\mathrm{~B}$ \\
\hline P0705 & Whittier Narrows 1987/10/01 14:42 & 6 & 30.1 & $\mathrm{~B}$ & $\mathrm{~B}$ \\
\hline
\end{tabular}


Table C. 3. Ground motion records for LMSR in Group 1

\begin{tabular}{|c|l|c|c|c|c|}
\hline \multirow{2}{*}{ Record ID } & \multicolumn{1}{|c|}{ Event and Time } & $\mathrm{M}$ & $\mathrm{R}$ & $\mathrm{GM}$ & USGS \\
\hline P0734 & Loma Prieta 1989/10/18 00:05 & 6.9 & 21.8 & $\mathrm{~A}$ & - \\
\hline P0749 & Loma Prieta 1989/10/18 00:05 & 6.9 & 17.9 & $\mathrm{~A}$ & $\mathrm{E}$ \\
\hline P0498 & Nahanni, Canada 1985/12/23 & 6.8 & 16 & $\mathrm{~A}$ & - \\
\hline P0885 & Northridge 1994/01/17 12:31 & 6.7 & 26.8 & $\mathrm{~A}$ & $\mathrm{~A}$ \\
\hline P0915 & Northridge 1994/01/17 12:31 & 6.7 & 22.7 & $\mathrm{~A}$ & $\mathrm{~A}$ \\
\hline P0937 & Northridge 1994/01/17 12:31 & 6.7 & 24.5 & $\mathrm{~A}$ & $\mathrm{~B}$ \\
\hline P0059 & San Fernando 1971/02/09 14:00 & 6.6 & 27 & $\mathrm{~A}$ & - \\
\hline P0076 & San Fernando 1971/02/09 14:00 & 6.6 & 24.2 & $\mathrm{~A}$ & $\mathrm{~B}$ \\
\hline P0077 & San Fernando 1971/02/09 14:00 & 6.6 & 23.5 & $\mathrm{~A}$ & $\mathrm{~A}$ \\
\hline P0084 & San Fernando 1971/02/09 14:00 & 6.6 & 19.1 & $\mathrm{~A}$ & - \\
\hline P0090 & San Fernando 1971/02/09 14:00 & 6.6 & 27.5 & $\mathrm{~A}$ & - \\
\hline P0738 & Loma Prieta 1989/10/18 00:05 & 6.9 & 19.9 & $\mathrm{~B}$ & $\mathrm{~B}$ \\
\hline P0746 & Loma Prieta 1989/10/18 00:05 & 6.9 & 24.2 & $\mathrm{~B}$ & $\mathrm{C}$ \\
\hline P0791 & Loma Prieta 1989/10/18 00:05 & 6.9 & 18.1 & $\mathrm{~B}$ & - \\
\hline P0906 & Northridge 1994/01/17 12:31 & 6.7 & 23.7 & $\mathrm{~B}$ & $\mathrm{~B}$ \\
\hline P0910 & Northridge 1994/01/17 12:31 & 6.7 & 20 & $\mathrm{~B}$ & $\mathrm{~B}$ \\
\hline P0950 & Northridge 1994/01/17 12:31 & 6.7 & 26.2 & $\mathrm{~B}$ & $\mathrm{~B}$ \\
\hline P0994 & Northridge 1994/01/17 12:31 & 6.6 & 24.9 & $\mathrm{~B}$ & $\mathrm{~B}$ \\
\hline P0056 & San Fernando 1971/02/09 14:00 & 6.6 & 20.3 & $\mathrm{~B}$ & $\mathrm{~B}$ \\
\hline P0078 & San Fernando 1971/02/09 14:00 & & & \\
\hline
\end{tabular}


Table C. 4. Ground motion records for LMLR in Group 1

\begin{tabular}{|c|l|c|c|c|c|}
\hline \multirow{2}{*}{ Record ID } & \multicolumn{1}{|c|}{ Event and Time } & $\mathrm{M}$ & $\mathrm{R}$ & $\mathrm{GM}$ & USGS \\
\hline P0880 & Landers 1992/06/28 11:58 & 7.3 & 42.2 & $\mathrm{~A}$ & $\mathrm{~A}$ \\
\hline P0768 & Loma Prieta 1989/10/18 00:05 & 6.9 & 30.6 & $\mathrm{~A}$ & $\mathrm{~A}$ \\
\hline P0771 & Loma Prieta 1989/10/18 00:05 & 6.9 & 44.8 & $\mathrm{~A}$ & $\mathrm{~A}$ \\
\hline P0774 & Loma Prieta 1989/10/18 00:05 & 6.9 & 36.3 & $\mathrm{~A}$ & $\mathrm{C}$ \\
\hline P0926 & Northridge 1994/01/17 12:31 & 6.7 & 36.1 & $\mathrm{~A}$ & $\mathrm{~A}$ \\
\hline P0965 & Northridge 1994/01/17 12:31 & 6.7 & 37 & $\mathrm{~A}$ & - \\
\hline P0969 & Northridge 1994/01/17 12:31 & 6.7 & 32.3 & $\mathrm{~A}$ & $\mathrm{~B}$ \\
\hline P0970 & Northridge 1994/01/17 12:31 & 6.7 & 34.6 & $\mathrm{~A}$ & $\mathrm{~B}$ \\
\hline P1011 & Northridge 1994/01/17 12:31 & 6.7 & 41.7 & $\mathrm{~A}$ & $\mathrm{~A}$ \\
\hline P0731 & Spitak, Armenia 1988/12/07 & 6.8 & 30 & $\mathrm{~A}$ & - \\
\hline P0811 & Cape Mendocino 1992/04/25 18:06 & 7.1 & 33.8 & $\mathrm{~B}$ & $\mathrm{~B}$ \\
\hline P0740 & Loma Prieta 1989/10/18 00:05 & 6.9 & 43 & $\mathrm{~B}$ & $\mathrm{~B}$ \\
\hline P0750 & Loma Prieta 1989/10/18 00:05 & 6.9 & 34.7 & $\mathrm{~B}$ & $\mathrm{~B}$ \\
\hline P0763 & Loma Prieta 1989/10/18 00:05 & 6.9 & 43.4 & $\mathrm{~B}$ & - \\
\hline P0793 & Loma Prieta 1989/10/18 00:05 & 6.9 & 39.9 & $\mathrm{~B}$ & $\mathrm{~B}$ \\
\hline P0903 & Northridge 1994/01/17 12:31 & 6.7 & 31.3 & $\mathrm{~B}$ & $\mathrm{~B}$ \\
\hline P0923 & Northridge 1994/01/17 12:31 & 6.7 & 35.2 & $\mathrm{~B}$ & $\mathrm{~B}$ \\
\hline P0973 & Northridge 1994/01/17 12:31 & 6.7 & 32.3 & $\mathrm{~B}$ & - \\
\hline P1014 & Northridge 1994/01/17 12:31 & 6.7 & 43.4 & $\mathrm{~B}$ & $\mathrm{~A}$ \\
\hline P0085 & San Fernando 1971/02/09 14:00 & & & & \\
\hline
\end{tabular}


Table C. 5. Ground motion records for NF in Group 1

\begin{tabular}{|c|l|c|c|c|c|}
\hline Record ID & \multicolumn{1}{|c|}{ Event and Time } & M & R & GM & USGS \\
\hline P0806 & Cape Mendocino 1992/04/25 18:06 & 7.1 & 8.5 & A & A \\
\hline P0873 & Landers 1992/06/28 11:58 & 7.3 & 1.1 & A & A \\
\hline P0733 & Loma Prieta 1989/10/18 00:05 & 6.9 & 11.2 & A & A \\
\hline P0760 & Loma Prieta 1989/10/18 00:05 & 6.9 & 10.3 & A & - \\
\hline P0770 & Loma Prieta 1989/10/18 00:05 & 6.9 & 6.1 & $\mathrm{~A}$ & - \\
\hline P0449 & Morgan Hill 1984/04/24 21:15 & 6.2 & 0.1 & $\mathrm{~A}$ & - \\
\hline P0995 & Northridge 1994/01/17 12:31 & 6.7 & 8 & $\mathrm{~A}$ & - \\
\hline P0996 & Northridge 1994/01/17 12:31 & 6.7 & 8 & $\mathrm{~A}$ & $\mathrm{~A}$ \\
\hline P0034 & Parkfield 1966/06/28 04:26 & 6.1 & 9.9 & $\mathrm{~A}$ & $\mathrm{~B}$ \\
\hline P0691 & Whittier Narrows 1987/10/01 14:42 & 6 & 9 & $\mathrm{~A}$ & $\mathrm{~A}$ \\
\hline P1043 & Kobe 1995/01/16 20:46 & 6.9 & 0.6 & $\mathrm{~B}$ & $\mathrm{~B}$ \\
\hline P0745 & Loma Prieta 1989/10/18 00:05 & 6.9 & 5.1 & $\mathrm{~B}$ & $\mathrm{~B}$ \\
\hline P0764 & Loma Prieta 1989/10/18 00:05 & 6.9 & 11.6 & $\mathrm{~B}$ & $\mathrm{~B}$ \\
\hline P0453 & Morgan Hill 1984/04/24 21:15 & 6.2 & 11.8 & $\mathrm{~B}$ & $\mathrm{~B}$ \\
\hline P0928 & Northridge 1994/01/17 12:31 & 6.7 & 8.2 & $\mathrm{~B}$ & $\mathrm{~B}$ \\
\hline P1021 & Northridge 1994/01/17 12:31 & 6.7 & 14.6 & $\mathrm{~B}$ & $\mathrm{C}$ \\
\hline P0032 & Parkfield 1966/06/28 04:26 & 6.1 & 9.2 & $\mathrm{~B}$ & $\mathrm{C}$ \\
\hline P0082 & San Fernando 1971/02/09 14:00 & 6.6 & 2.8 & $\mathrm{~B}$ & - \\
\hline P0624 & Whittier Narrows 1987/10/01 14:42 & 6 & 12.1 & $\mathrm{~B}$ & - \\
\hline P0706 & Whittier Narrows 1987/10/01 14:42 & 6.5 & $\mathrm{~B}$ & $\mathrm{C}$ \\
\hline
\end{tabular}


Table C. 6. Ground motion records for SMSR in Group 2

\begin{tabular}{|c|l|c|c|c|c|}
\hline Record ID & \multicolumn{1}{|c|}{ Event and Time } & $\mathrm{M}$ & $\mathrm{R}$ & $\mathrm{GM}$ & $\mathrm{USGS}$ \\
\hline P0345 & Coalinga 1983/05/02 23:42 & 6.4 & 29.5 & $\mathrm{C}$ & - \\
\hline P0346 & Coalinga 1983/05/02 23:42 & 6.4 & 29.9 & $\mathrm{C}$ & - \\
\hline P0348 & Coalinga 1983/05/02 23:42 & 6.4 & 28.1 & $\mathrm{C}$ & - \\
\hline P0361 & Coalinga 1983/05/02 23:42 & 6.4 & 29.5 & $\mathrm{C}$ & - \\
\hline P0520 & N. Palm Springs 1986/07/08 09:20 & 6 & 15.8 & $\mathrm{C}$ & $\mathrm{B}$ \\
\hline P0525 & N. Palm Springs 1986/07/08 09:20 & 6 & 29.8 & $\mathrm{C}$ & $\mathrm{B}$ \\
\hline P0604 & Whittier Narrows 1987/10/01 14:42 & 6 & 25.5 & $\mathrm{C}$ & $\mathrm{B}$ \\
\hline P0617 & Whittier Narrows 1987/10/01 14:42 & 6 & 17.1 & $\mathrm{C}$ & $\mathrm{C}$ \\
\hline P0625 & Whittier Narrows 1987/10/01 14:42 & 6 & 19 & $\mathrm{C}$ & $\mathrm{C}$ \\
\hline P0647 & Whittier Narrows 1987/10/01 14:42 & 6 & 22.7 & $\mathrm{C}$ & $\mathrm{C}$ \\
\hline P0698 & Whittier Narrows 1987/10/01 14:42 & 6 & 27.5 & $\mathrm{C}$ & $\mathrm{B}$ \\
\hline P0555 & Chalfant Valley 1986/07/21 14:42 & 6.2 & 18.7 & $\mathrm{D}$ & - \\
\hline P0323 & Coalinga 1983/05/02 23:42 & 6.4 & 25.5 & $\mathrm{D}$ & - \\
\hline P0352 & Coalinga 1983/05/02 23:42 & 6.4 & 29.2 & $\mathrm{D}$ & - \\
\hline P0406 & Coalinga 1983/07/22 02:39 & 5.8 & 17.4 & $\mathrm{D}$ & - \\
\hline P0153 & Coyote Lake 1979/08/06 17:05 & 5.7 & 15.6 & $\mathrm{D}$ & $\mathrm{B}$ \\
\hline P0154 & Coyote Lake 1979/08/06 17:05 & 5.7 & 17.2 & $\mathrm{D}$ & $\mathrm{B}$ \\
\hline P0164 & Imperial Valley 1979/10/15 23:16 & 6.5 & 23.8 & $\mathrm{D}$ & $\mathrm{C}$ \\
\hline P0173 & Imperial Valley 1979/10/15 23:16 & 6.5 & 15.5 & $\mathrm{D}$ & $\mathrm{C}$ \\
\hline P0450 & Morgan Hill 1984/04/24 21:15 & 6.2 & 15.1 & $\mathrm{D}$ & $\mathrm{C}$ \\
\hline
\end{tabular}


Table C. 7. Ground motion records for SMLR in Group 2

\begin{tabular}{|c|l|c|c|c|c|}
\hline \multirow{2}{*}{ Record ID } & \multicolumn{1}{|c|}{ Event and Time } & $\mathrm{M}$ & $\mathrm{R}$ & $\mathrm{GM}$ & USGS \\
\hline P0328 & Coalinga 1983/05/02 23:42 & 6.4 & 43.9 & $\mathrm{C}$ & - \\
\hline P0340 & Coalinga 1983/05/02 23:42 & 6.4 & 31 & $\mathrm{C}$ & - \\
\hline P0367 & Coalinga 1983/05/02 23:42 & 6.4 & 41 & $\mathrm{C}$ & - \\
\hline P0152 & Coyote Lake 1979/08/06 17:05 & 5.7 & 31.2 & $\mathrm{C}$ & $\mathrm{C}$ \\
\hline P0217 & Livermore 1980/01/24 19:00 & 5.8 & 37.7 & $\mathrm{C}$ & $\mathrm{C}$ \\
\hline P0535 & N. Palm Springs 1986/07/08 09:20 & 6 & 32 & $\mathrm{C}$ & $\mathrm{B}$ \\
\hline P0667 & Whittier Narrows 1987/10/01 14:42 & 6 & 30.8 & $\mathrm{C}$ & $\mathrm{B}$ \\
\hline P0689 & Whittier Narrows 1987/10/01 14:42 & 6 & 37.7 & $\mathrm{C}$ & $\mathrm{C}$ \\
\hline P0549 & Chalfant Valley 1986/07/21 14:42 & 6.2 & 44.9 & $\mathrm{D}$ & - \\
\hline P0551 & Chalfant Valley 1986/07/21 14:42 & 6.2 & 37.2 & $\mathrm{D}$ & - \\
\hline P0324 & Coalinga 1983/05/02 23:42 & 6.4 & 41.6 & $\mathrm{D}$ & - \\
\hline P0337 & Coalinga 1983/05/02 23:42 & 6.4 & 36.4 & $\mathrm{D}$ & - \\
\hline P0189 & Imperial Valley 1979/10/15 23:16 & 6.5 & 31.7 & $\mathrm{D}$ & $\mathrm{C}$ \\
\hline P0447 & Morgan Hill 1984/04/24 21:15 & 6.2 & 32.5 & $\mathrm{D}$ & $\mathrm{C}$ \\
\hline P0455 & Morgan Hill 1984/04/24 21:15 & 6.2 & 30.3 & $\mathrm{D}$ & $\mathrm{B}$ \\
\hline P0512 & N. Palm Springs 1986/07/08 09:20 & 6 & 43.3 & $\mathrm{D}$ & $\mathrm{C}$ \\
\hline P0527 & N. Palm Springs 1986/07/08 09:20 & 6 & 38.2 & $\mathrm{D}$ & - \\
\hline P0539 & N. Palm Springs 1986/07/08 09:20 & 6 & 44.4 & $\mathrm{D}$ & - \\
\hline P0599 & Whittier Narrows 1987/10/01 14:42 & 6 & 38.9 & $\mathrm{D}$ & $\mathrm{B}$ \\
\hline P0623 & Whittier Narrows 1987/10/01 14:42 & 6 & 35 & $\mathrm{D}$ & $\mathrm{B}$ \\
\hline
\end{tabular}


Table C. 8. Ground motion records for LMSR in Group 2

\begin{tabular}{|c|l|c|c|c|c|}
\hline \multirow{2}{*}{ Record ID } & \multicolumn{1}{|c|}{ Event and Time } & $\mathrm{M}$ & $\mathrm{R}$ & $\mathrm{GM}$ & $\mathrm{USGS}$ \\
\hline P0810 & Cape Mendocino 1992/04/25 18:06 & 7.1 & 18.5 & $\mathrm{C}$ & $\mathrm{B}$ \\
\hline P0817 & Landers 1992/06/28 11:58 & 7.3 & 19.3 & $\mathrm{C}$ & $\mathrm{B}$ \\
\hline P0889 & Northridge 1994/01/17 12:31 & 6.7 & 20.8 & $\mathrm{C}$ & $\mathrm{B}$ \\
\hline P0890 & Northridge 1994/01/17 12:31 & 6.7 & 19.6 & $\mathrm{C}$ & $\mathrm{B}$ \\
\hline P0891 & Northridge 1994/01/17 12:31 & 6.7 & 24 & $\mathrm{C}$ & $\mathrm{B}$ \\
\hline P0916 & Northridge 1994/01/17 12:31 & 6.7 & 22.3 & $\mathrm{C}$ & $\mathrm{C}$ \\
\hline P0933 & Northridge 1994/01/17 12:31 & 6.7 & 17.7 & $\mathrm{C}$ & $\mathrm{B}$ \\
\hline P0975 & Northridge 1994/01/17 12:31 & 6.7 & 22.8 & $\mathrm{C}$ & $\mathrm{B}$ \\
\hline P0058 & San Fernando 1971/02/09 14:00 & 6.6 & 25.8 & $\mathrm{C}$ & - \\
\hline P0808 & Cape Mendocino 1992/04/25 18:06 & 7.1 & 23.6 & $\mathrm{D}$ & $\mathrm{B}$ \\
\hline P0814 & Landers 1992/06/28 11:58 & 7.3 & 23.2 & $\mathrm{D}$ & $\mathrm{B}$ \\
\hline P0818 & Landers 1992/06/28 11:58 & 7.3 & 24.2 & $\mathrm{D}$ & $\mathrm{B}$ \\
\hline P0865 & Landers 1992/06/28 11:58 & 7.3 & 21.2 & $\mathrm{D}$ & $\mathrm{B}$ \\
\hline P0881 & Landers 1992/06/28 11:58 & 7.3 & 24.9 & $\mathrm{D}$ & $\mathrm{C}$ \\
\hline P0732 & Loma Prieta 1989/10/18 00:05 & 6.9 & 28.2 & $\mathrm{D}$ & $\mathrm{C}$ \\
\hline P0737 & Loma Prieta 1989/10/18 00:05 & 6.9 & 16.1 & $\mathrm{D}$ & $\mathrm{C}$ \\
\hline P0742 & Loma Prieta 1989/10/18 00:05 & 6.9 & 28.2 & $\mathrm{D}$ & $\mathrm{C}$ \\
\hline P0743 & Loma Prieta 1989/10/18 00:05 & 6.9 & 21.4 & $\mathrm{D}$ & $\mathrm{B}$ \\
\hline P0884 & Northridge 1994/01/17 12:31 & 6.7 & 25.5 & $\mathrm{D}$ & $\mathrm{C}$ \\
\hline P0905 & Northridge 1994/01/17 12:31 & & & & \\
\hline
\end{tabular}


Table C. 9. Ground motion records for LMLR in Group 2

\begin{tabular}{|c|l|c|c|c|c|}
\hline \multirow{2}{*}{ Record ID } & \multicolumn{1}{|c|}{ Event and Time } & $\mathrm{M}$ & $\mathrm{R}$ & $\mathrm{GM}$ & $\mathrm{USGS}$ \\
\hline P0907 & Northridge 1994/01/17 12:31 & 6.7 & 32.8 & $\mathrm{C}$ & $\mathrm{B}$ \\
\hline P0918 & Northridge 1994/01/17 12:31 & 6.7 & 36.3 & $\mathrm{C}$ & $\mathrm{C}$ \\
\hline P0921 & Northridge 1994/01/17 12:31 & 6.7 & 38.3 & $\mathrm{C}$ & $\mathrm{C}$ \\
\hline P0924 & Northridge 1994/01/17 12:31 & 6.7 & 42 & $\mathrm{C}$ & $\mathrm{C}$ \\
\hline P0999 & Northridge 1994/01/17 12:31 & 6.7 & 39.2 & $\mathrm{C}$ & $\mathrm{C}$ \\
\hline P0807 & Cape Mendocino 1992/04/25 18:06 & 7.1 & 44.6 & $\mathrm{D}$ & $\mathrm{B}$ \\
\hline P0860 & Landers 1992/06/28 11:58 & 7.3 & 36.1 & $\mathrm{D}$ & $\mathrm{B}$ \\
\hline P0773 & Loma Prieta 1989/10/18 00:05 & 6.9 & 36.1 & $\mathrm{D}$ & - \\
\hline P0778 & Loma Prieta 1989/10/18 00:05 & 6.9 & 32.6 & $\mathrm{D}$ & $\mathrm{C}$ \\
\hline P0896 & Northridge 1994/01/17 12:31 & 6.7 & 40.7 & $\mathrm{D}$ & $\mathrm{C}$ \\
\hline P0904 & Northridge 1994/01/17 12:31 & 6.7 & 30.9 & $\mathrm{D}$ & $\mathrm{C}$ \\
\hline P0912 & Northridge 1994/01/17 12:31 & 6.7 & 37.9 & $\mathrm{D}$ & $\mathrm{B}$ \\
\hline P0914 & Northridge 1994/01/17 12:31 & 6.7 & 30 & $\mathrm{D}$ & $\mathrm{C}$ \\
\hline P0920 & Northridge 1994/01/17 12:31 & 6.7 & 42.4 & $\mathrm{D}$ & $\mathrm{C}$ \\
\hline P0929 & Northridge 1994/01/17 12:31 & 6.7 & 34.2 & $\mathrm{D}$ & $\mathrm{B}$ \\
\hline P0931 & Northridge 1994/01/17 12:31 & 6.7 & 35.1 & $\mathrm{D}$ & $\mathrm{B}$ \\
\hline P0938 & Northridge 1994/01/17 12:31 & 6.7 & 42.5 & $\mathrm{D}$ & $\mathrm{C}$ \\
\hline P0942 & Northridge 1994/01/17 12:31 & 6.7 & 35.7 & $\mathrm{D}$ & $\mathrm{B}$ \\
\hline P0944 & Northridge 1994/01/17 12:31 & 6.7 & 38.4 & $\mathrm{D}$ & $\mathrm{C}$ \\
\hline P0946 & Northridge 1994/01/17 12:31 & 44.2 & $\mathrm{D}$ & $\mathrm{C}$ \\
\hline
\end{tabular}


Table C. 10. Ground motion records for NF in Group 2

\begin{tabular}{|c|l|c|c|c|c|}
\hline Record ID & \multicolumn{1}{|c|}{ Event and Time } & $\mathrm{M}$ & $\mathrm{R}$ & $\mathrm{GM}$ & USGS \\
\hline P0190 & Imperial Valley 1979/10/15 23:16 & 6.5 & 11.1 & $\mathrm{C}$ & $\mathrm{C}$ \\
\hline P0816 & Landers 1992/06/28 11:58 & 7.3 & 11.6 & $\mathrm{C}$ & $\mathrm{B}$ \\
\hline P0744 & Loma Prieta 1989/10/18 00:05 & 6.9 & 4.5 & $\mathrm{C}$ & $\mathrm{C}$ \\
\hline P0454 & Morgan Hill 1984/04/24 21:15 & 6.2 & 3.4 & $\mathrm{C}$ & $\mathrm{C}$ \\
\hline P0528 & N. Palm Springs 1986/07/08 09:20 & 6 & 10.1 & $\mathrm{C}$ & $\mathrm{B}$ \\
\hline P0541 & N. Palm Springs 1986/07/08 09:20 & 6 & 7.3 & $\mathrm{C}$ & $\mathrm{A}$ \\
\hline P0988 & Northridge 1994/01/17 12:31 & 6.7 & 14.6 & $\mathrm{C}$ & $\mathrm{B}$ \\
\hline P1005 & Northridge 1994/01/17 12:31 & 6.7 & 7.1 & $\mathrm{C}$ & $\mathrm{C}$ \\
\hline P0636 & Whittier Narrows 1987/10/01 14:42 & 6 & 11.4 & $\mathrm{C}$ & $\mathrm{B}$ \\
\hline P0648 & Whittier Narrows 1987/10/01 14:42 & 6 & 13.5 & $\mathrm{C}$ & $\mathrm{C}$ \\
\hline P0809 & Cape Mendocino 1992/04/25 18:06 & 7.1 & 9.5 & $\mathrm{D}$ & $\mathrm{C}$ \\
\hline P0553 & Chalfant Valley 1986/07/21 14:42 & 6.2 & 9.2 & $\mathrm{D}$ & - \\
\hline P0368 & Coalinga 1983/05/02 23:42 & 6.4 & 8.5 & $\mathrm{D}$ & - \\
\hline P0006 & Imperial Valley 1940/05/19 04:37 & 7 & 8.3 & $\mathrm{D}$ & $\mathrm{C}$ \\
\hline P0160 & Imperial Valley 1979/10/15 23:16 & 6.5 & 12.9 & $\mathrm{D}$ & - \\
\hline P0736 & Loma Prieta 1989/10/18 00:05 & 6.9 & 14.4 & $\mathrm{D}$ & $\mathrm{C}$ \\
\hline P0451 & Morgan Hill 1984/04/24 21:15 & 6.2 & 14.2 & $\mathrm{D}$ & $\mathrm{C}$ \\
\hline P0452 & Morgan Hill 1984/04/24 21:15 & 6.2 & 12.8 & $\mathrm{D}$ & $\mathrm{C}$ \\
\hline P0530 & N. Palm Springs 1986/07/08 09:20 & 6 & 8.2 & $\mathrm{D}$ & $\mathrm{B}$ \\
\hline P0893 & Northridge 1994/01/17 12:31 & 6.7 & 13 & $\mathrm{D}$ & $\mathrm{C}$ \\
\hline
\end{tabular}




\section{APPENDIX D}

Bae, B. H., Nam, S. I., Kwon, K. J., Song, H. Y., and Kim, M. H. (1992). "An experimental study on the workability and mix proportions design of flowing concrete using superplasticizing agents." Proceedings of the Architecture Institute of Korea, 12(1), 435-438.

Cho, B. Y., Yoon, G. W., Han, C. K., and Bahn, H. Y. (1994). "The influence of the properties of high strength concrete with the qualities of cement and aggregates." Proceedings of the Architecture Institute of Korea, 14(2), 669-674.

Cho, M. H., Khil, B. S., Chae, Y. S., and Nam, J. H. (1998). "An experimental study on the compressive strength properties of mass concrete with fly ash." Proceedings of the Architecture Institute of Korea, 18(2), 678-683.

Choi, J. S., Yoon, B. S., Lee, A. B., Kim, J. M., and Kim, M. H. (1993). "An experimental study on the influence of fly ash affecting workability and engineering properties of ultra high strength concrete." Proceedings of the Architecture Institute of Korea, 13(1), 439-442.

Kang, B. H., Son, J. W., and Kim, S. H. (1994). "An experimental study on the influence of admixture additive materials for high performance concrete." Proceedings of the Architecture Institute of Korea, 14(2), 659-662.

Kim, G. S., Kim, J. M., Kim, Y. K., and Kim, M. H. (1989). "An experimental study on the mechanical properties of high strength concrete using a superplasticizer." Proceedings of the Architecture Institute of Korea, 9(1), 485-488.

Kim, G. S., Kwon, Y. J., Kim, J. W., Moon, J. S., and Kim, M. H. (1991). "An experimental study on the influence of superplasticizing agents and coarse aggregates on fluidity performance and engineering properties of high strength flowing concrete." Proceedings of the Architecture Institute of Korea, 11(2), 487490.

Kim, G. Y., Kim, J. M., Nam, S. I., and Kim, M. H. (1995). "An experimental study in the influence of the cement content and sand aggregate ratio affecting the workability and engineering properties of the high strength concrete." Proceedings of the Architecture Institute of Korea, 15(1), 587-592.

Kim, J. M., Nam, S. I., and Kim, M. H. (1988). "An experimental study on the mechanical properties of high strength concrete using superplasticizers." Proceedings of the Architecture Institute of Korea, 8(1), 611-616. 
Kim, J. W., Lim, J. S., Kim, G. S., Nam, S. I., and Kim, M. H. (1991). "An experimental study on the influence on the kinds of coarse aggregate on engineering properties of high strength concrete." Proceedings of the Architecture Institute of Korea, 11(2), 607-612.

Kwon, I. K., Kang, B. H., and Oh, C. H. (1989). "An experimental study on the nondestructive testing method for the inference on the compressive strength of concrete." Proceedings of the Architecture Institute of Korea, 9(1), 395-398.

Kwon, K. J., Song, D. C., Nam, S. I., Song, H. Y., and Kim, M. H. (1991). "An experimental study on the fluidity performance and engineering properties in concrete structures." Proceedings of the Architecture Institute of Korea, 11(2), 491494.

Nam, S. I., Kim, G. S., Kwon, K. W., and Kim, M. H. (1989). "A fundamental study on the influence of cement content to engineering properties of high strength concrete." Proceedings of the Architecture Institute of Korea, 9(1), 477-480.

Nam, S. I., Kim, J. M., and Kim, M. H. (1988). "A fundamental study on the influence of cement content to engineering properties of high strength concrete." Proceedings of the Architecture Institute of Korea, 8(2), 663-668.

OH, S. K., OH, S. K., Song, H. K., and Kim, M. H. (1988). "An experimental study on the workability and strength properties of the flowing concrete by different kinds of aggregate." Proceedings of the Architecture Institute of Korea, 8(1), 573-576.

Ryu, J. Y., Yang, C. H., Suhr, M. S., and Kim, M. H. (1989). "A study on the effect of curing condition on mechanical properties of concrete." Proceedings of the Architecture Institute of Korea, 9(1), 481-484.

Song, H. Y., Kim, J. M., Kwon, I. K., and Kim, M. H. (1989). "A fundamental study on the improvement of concrete properties for nuclear power plants by using superplasticiaing agents." Proceedings of the Architecture Institute of Korea, 9(1), 489-490.

Suhr, M. S., OH, S. K., Song, H. Y., and Kim, M. H. (1988). "An experimental study on the workability and strength properties of the flowing concrete by different kinds of aggregate." Proceedings of the Architecture Institute of Korea, 8(1), 569-572.

Suhr, M. S., OH, S. K., Song, H. Y., and Kim, M. H. (1988). "An experimental study on the workability and strength properties of the flowing concrete by different kinds of aggregate." Proceedings of the Architecture Institute of Korea, 8(2), 691-694. 
Yoon, B. S., Song, K. H., Lee, S. S., Kim, J. M., Nam, S. I., and Kim, M. H. (1993). "An experimental study on the workability and engineering properties of concrete mixed fly ash." Proceedings of the Architecture Institute of Korea, 13(1), 435-438.

Yoon, G. W., Cho, B. Y., Han, C. G., and Bahn, H. Y. (1994). "The influence of the properties of high strength concrete with the qualities of cement and aggregate." Proceedings of the Architecture Institute of Korea, 14(1), 901-908. 


\section{VITA}

Name: $\quad$ Qindan Huang

Address: $\quad$ Department of Civil Engineering

Texas A\&M University

3136 TAMU

College Station, TX, 77843-3136

Email Address: qindan_h@tamu.edu

qindan_h@hotmail.edu

Education: $\quad$ B.S., Civil Engineering, Tongji University, Shanghai, China, 2001 M.S., Civil Engineering, University of Toledo, OH, USA, 2004

Ph.D., Civil Engineering, Texas A\&M University, College Station, TX, 2010 